\title{
Spektrale Kontrolle von elastischer Dynamik und Wärmetransport in periodischen Nanostrukturen
}

\author{
Dissertation \\ zur Erlangung des mathematisch-naturwissenschaftlichen Doktorgrades \\ "Doctor rerum naturalium" \\ der Georg-August-Universität Göttingen \\ im Promotionsprogramm Physik \\ der Georg-August University School of Science (GAUSS) \\ vorgelegt von \\ Dennis Meyer
}

aus Osterode am Harz

Göttingen, 2021 
$\underline{\text { Betreuungsausschuss }}$

Prof. Dr. Stefan Mathias, I. Physikalisches Institut

Dr. Henning Ulrichs, I. Physikalisches Institut

Prof. Dr. Christian Jooss, Institut für Materialphysik

apl. Prof. Dr. Vasily Moshnyaga, I. Physikalisches Institut

Mitglieder der Prüfungskommission

Referent: Dr. Henning Ulrichs, I. Physikalisches Institut

Korreferent: Prof. Dr. Christian Jooss, Institut für Materialphysik

Weitere Mitglieder der Prüfungskommission:

apl. Prof. Dr. Vasily Moshnyaga, I. Physikalisches Institut

Prof. Dr. Matthias Krüger, Institut für Theoretische Physik

Dr. Daniel Steil, I. Physikalisches Institut

Dr. Oliver Bünermann, Institut für Physikalische Chemie

Tag der mündlichen Prüfung: 27.09.2021 




\section{Inhaltsverzeichnis}

1 Einleitung 1

1.1 Motivation: Von Schall, Ultraschall, Licht und Wärme . . . . . . . . 1

1.2 Stand der Forschung . . . . . . . . . . . . . . . . . . 5

1.3 Umfang dieser Arbeit . . . . . . . . . . . . . . . . . . . 8

2 Theorie 11

$2.1 \mathrm{GHz}-$ und THz-Gitterschwingungen . . . . . . . . . . . . . . . 11

2.2 Phononen . . . . . . . . . . . . . . . . . . . 22

2.2.1 Dispersion . . . . . . . . . . . . . . . . . 23

2.2.2 Wärmeleitfähigkeit der Phononen . . . . . . . . . . . . 26

2.2.3 Born-von-Kármán-Modell . . . . . . . . . . . . . . . . 31

3 Experimentelle Methoden $\quad 39$

3.1 Probenherstellung . . . . . . . . . . . . . . . . . . . . . 40

3.1 .1 Probenübersicht . . . . . . . . . . . . . . . 40

3.1.2 Pulsed Laser Deposition . . . . . . . . . . . . . . 43

3.1.3 Metallorganische Aerosoldeposition (MAD) . . . . . . . . . 43

3.1.4 UHV-Elektronenstrahlverdampfen . . . . . . . . . . . . . . 44

3.2 Numerische Simulationen . . . . . . . . . . . . . . . . . . . 46

3.2.1 Born-von-Kármán Simulation . . . . . . . . . . . . . 47

3.3 Pump-Probe-Reflektometrie (PPR) . . . . . . . . . . . . . . . . . . 49

3.4 Messmethode zur Wärmeleitfähigkeitsmessung . . . . . . . . . . . . 52

3.4.1 Transiente Thermoreflektivität (TTR) . . . . . . . . . 53

3.4.1.1 TTR-Aufbau .............. 54

3.4.1.2 Auswertung von TTR-Messdaten . . . . . . . . . 56

3.4.1.3 Temperaturabhängige Transiente Thermoreflektivität $(\mathrm{TTTR}) \ldots \ldots \ldots \ldots$. . . . . . . 6 67

3.4.1.4 Evaluation des TTR-Aufbaus . . . . . . . . 68 
4 Ergebnisse $\quad 73$

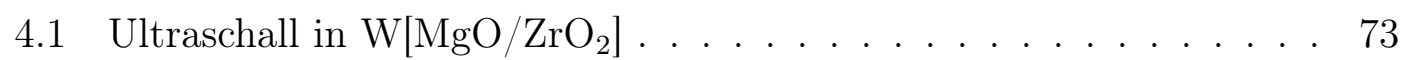

4.1.1 PPR Ergebnisse . . . . . . . . . . . . . . . . . . 74

4.1.2 Finite-Differenzen-Simulation . . . . . . . . . . . . . 79

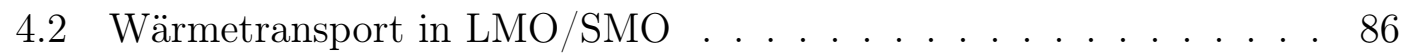

4.2.1 TTR an $\mathrm{LMO}_{m} / \mathrm{SMO}_{n}$-Übergittern . . . . . . . . . . . . . 87

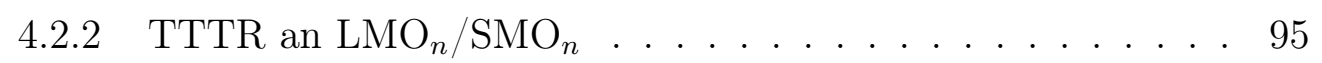

4.2.3 Wärmetransport in LMO/SMO mit AFM-Phase . . . . . . . 102

5 Zusammenfassung und Ausblick 107

5.1 Ultraschalldynamik in Multilagen . . . . . . . . . . . . . . . . 107

5.2 Magnetoelastische Kopplung in LSMO/(LMO/SMO) . . . . . . . . 108

5.3 Wärmetransport in Übergittern . . . . . . . . . . . . . . . . . . 111

5.3.1 Ausblick: Phononenisolator . . . . . . . . . . . . 116

$\begin{array}{ll}\text { Danksagung } & 119\end{array}$

$\begin{array}{lr}\text { Abgrenzung } & 121\end{array}$

$\begin{array}{ll}\text { Abkürzungsverzeichnis } & 123\end{array}$

$\begin{array}{lr}\text { Literaturverzeichnis } & 125\end{array}$

$\begin{array}{lr}\text { A Anhang } & 149\end{array}$

A.1 Materialparameter . . . . . . . . . . . . . . . . . . 149

A.2 Determinaten zur Herleitung der Rytov-Gleichung . . . . . . . . . . 152

A.3 TTR-Aufbau . . . . . . . . . . . . . . . . . . . . 155

A.3.1 Entwicklung des TTR-Aufbaus . . . . . . . . . . . 155

A.3.2 TTR-Messanleitung . . . . . . . . . . . . . . 157

A.4 Anleitung: Elektronenstrahlverdampfen . . . . . . . . . . . . . . . 159

A.5 Simulation mit dem Born-Von-Kármán-Modell . . . . . . . . . . . . 162

A.6 Phononendispersion aus dem BK-Modell . . . . . . . . . . . . . . 165 


\section{Kapitel 1}

\section{Einleitung}

Considering it as thus established, that heat is not a substance, but a dynamical form of mechanical effect, we perceive that there must be an equivalence between mechanical work and heat, as between cause and effect.

Baron William Thomson Kelvin [1]

\subsection{Motivation: Von Schall, Ultraschall, Licht und Wärme}

Das Auslegen und Simulieren der Akustik eines Raumes ist schon seit Jahrzehnten $[2,3]$ ein fester Bestandteil bei der Einrichtung von z.B. Tonstudios, Clubs oder Konzerthallen. Mit Hilfe von Computersimulationen kann das frequenzabhängige Reflexionsverhalten eines Raumes bereits in der Planung berücksichtigt werden oder mit empfindlichen, linearen Mikrofonen und einer Signal-Echo-Messung im Nachhinein vermessen werden. Sollten nicht die gewünschten Eigenschaften vorliegen, so gibt es sowohl kommerzielle als auch Bastellösungen um den Raumschall zu beeinflussen: So sieht man in Proberäumen und Tonstudios häufig schwere Teppiche, Decken und Vorhänge. Diese dienen dazu, hochfrequenten Schall zu absorbieren, um einen Raum weniger hell und leer klingen zu lassen. Von Eierkartons an den Wänden erhoffen sich Hobbymusiker eine Reduktion von Echos durch die Zerstreuung von Schall zwischen den Kartonkegeln. Auch ein sperriges Sofa wird gerne genutzt, um tiefe Frequenzen an ihrer Ausbreitung zu hindern. Neben diesen einfachen Mitteln der Schallabsorption, gibt es aber auch deutlich komplexere 
Lösungen, welche die Aufgabe haben, genau definierte Frequenzbereiche zu absorbieren, zu reflektieren oder zu zerstreuen. Dazu gehören zum Beispiel mit Mineralwolle gefüllte Absorber oder sogenannte Skyscraper-Diffusoren [2] (beispielhaft zu sehen in Abbildung 1.1 (a)), die durch gezielte Streuung für ein ausgeglichenes Klangbild im Raum sorgen.

(a)

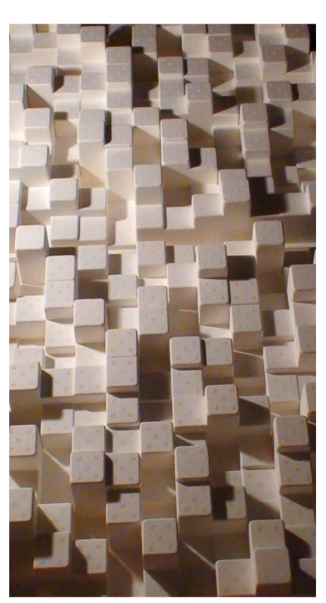

(b)

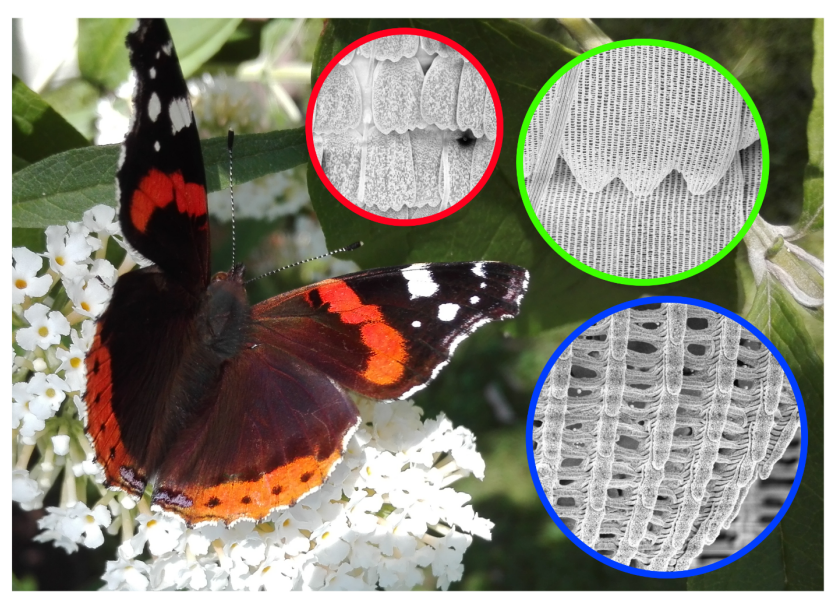

Abbildung 1.1: (a) Ein sogenannter Skyscraper-Diffusor aus Schaumstoff zur gezielten Streuung bestimmter Schallfrequenzen. Quelle: Wikimedia Commons [4]. (b) Das Schimmern der Farbe eines Schmetterlings (im Bild: Vanessa atalanta „Admiral", eigenes Werk) entsteht durch Licht-Interferenzeffekte an den Nanostrukturen der Flügel. Als Insets sind REM Aufnahmen eines Flügels in 140-facher (Rot), 1.000-facher (grün) und 10.000-facher Vergrößerung (blau) zu sehen.

Ähnliche Beispiele für Wellenmechanik und Interferenz finden wir im Bereich der Optik: Die Flügel von Schmetterlingen sind so strukturiert (Abb. 1.1 (b)), dass unter bestimmten Einfallsrichtungen nur spezifische Wellenlängen des Lichtes reflektiert werden [5, 6]. Der Glasflügelfalter hat eine besondere Flügelstruktur: Die Nanostrukturierung seiner Flügel sorgt für eine hohe Lichttransmission im gesamten sichtbaren Spektrum, auch unter großen Einfallswinkeln von bis zu 80 Grad $[7,8]$. Weiterhin machen wir uns auch in technischen Anwendungen den Wellencharakter des Lichtes mit Hilfe von Nanostrukturierung zu Nutzen: Durch das Stapeln von dünnen Schichten mit unterschiedlichen Brechungsindizes entsteht bei Reflexion und Transmission von Licht Interferenz und es lassen sich sehr schmalbandige Bragg-Spiegel oder Antireflexionsbeschichtungen herstellen.[9] 
Nach dieser kleinen Einleitung möchte ich nun auf dem Zusammenhang von solchen Wellenphänomenen mit dem eigentlichen Thema dieser Arbeit zu sprechen kommen:

In einem Festkörper nehmen alle vorhandenen Freiheitsgrade einen Teil der thermische Energie auf. In einem Metall sind dies Elektronen und die Bewegungen der Gitteratome. In einem Isolator sind es nur die Gitterschwingungen. Im Rahmen der quantenmechanischen Quasiteilchenbeschreibung bezeichnen wir solche Gitterschwingungen auch als Phononen. In einem dielektrischen Kristall können wir bei der Ausbreitung der Wärme den Anteil der Elektronen vernachlässigen und kommen zu der Schlussfolgerung, dass die Wärme durch eine Ausbreitung der Gitterschwingungen im Kristall erfolgen muss. Doch während wir Phononen und Ultraschall mit den Gesetzen der Wellenmechanik beschreiben, nutzen wir im praktischen Alltag eine Diffusionsgleichungen für die Ausbreitung von Wärme. Diese ursprünglich empirische Beschreibung rechtfertigt sich dadurch, dass die typischen Phononenrelaxationszeiten bei Raumtemperatur durch Streuung an Grenzflächen, Defekten und durch Phonon-Phonon-Streuung klein sind. Wir können an keinem Ort unserer Proben zeitlich kohärente (Schall-)Wellen beobachten, welche aber für das Auftreten von Interferenzeffekten unabdingbar sind.[10, 11] Liegt ein Temperaturgradient an, transportieren die Gitterschwingungen die Wärme von der heißen zur kalten Seite. Ihr Weg ist aber durch Streuung dominiert und folgt daher einem Random-Walk der durch das Fick'sche Diffusionsgesetz beschrieben wird [12].

Doch unter welchen Bedingungen treten die Welleneigenschaften der Phononen beim Wärmetransport zu Tage? Der sogenannte „Zweite Schall“ ist ein solches Experiment und eines der ersten, welches die Verknüpfung zwischen Wärmetransport und Wellenmechanik der Phononen gezeigt hat [12-15]: Wird bei tiefen Temperaturen in sehr reinen Kristallen, z.B. durch einen Laserpuls an der Oberfläche ein Temperaturpuls angeregt, so können sich die angeregten Phononen ballistisch im Kristall ausbreiten. Eine Phononendichtewelle läuft durch die Probe und kann mittels Licht-Phonon-Streuung oder als Wärmepuls ${ }^{1}$ detektiert werden. Dieses Wellenpaket aus Gitterschwingungen, also einer Dichtewelle aus Phononen, wird Zweiter Schall genannt.

\footnotetext{
${ }^{1}$ Eine lokal erhöhte Phononendichte entspricht auch einer lokal erhöhten Temperatur und eine erniedrigte Phononendichte einer lokal herabgesetzten Temperatur.[14]
} 
Wenn sich der Wärmetransport unter bestimmten Bedingungen also auf die Ausbreitung kohärenter Gitterschwingungen zurückführen lässt, stellen sich folgende Fragen: Gibt es Materialien und Probensysteme, in denen ballistischer Phononentransport auch bei Raumtemperatur einen messbaren Einfluss auf die Wärmeleitfähigkeit hat und können wir mit den bekannten Werkzeugen der Wellenoptik und Akustik Einfluss auf diesen Transport nehmen?

Mit letzterem eröffnen sich weitreichende und spannende Anwendungsgebiete, darunter zum Beispiel Bragg-ähnliche akustische Spiegel, welche Phononen und den mit ihnen verbundenen Wärmetransport lenken. So könnte die Effizienz von vormals durch Wärmediffusion limitierte Anwendungen, wie thermoelektrische Geräte oder Brennkammern, erhöht werden [16-18]. Eine aktive Kontrolle der Wärmediffusion könnte es außerdem erlauben, thermische Leiterbahnen und Dioden zu konstruieren, die als Grundbausteine für thermische Logikschaltkreise dienen [19, 20]. Auf diese Weise könnte die zur Zeit noch ungenutzte Abwärme von Computerchips nutzbar gemacht werden.

Neben den Einfluss auf den Wärmetransport in Nanostrukturen eröffnet die Kontrolle kohärenter Phononen noch weitere potentielle Anwendungen. Beispielsweise könnte eine Quelle für kohärente Phononen im THz-Bereich (ein sogenannter Phononenlaser) [21, 22] und die räumliche Kontrolle der Phononenemission [23] neue Experimente für die Grundlagenforschung in Bereichen wie Phononik und Optomechanik ermöglichen [22, 24]. Desweiteren könnte durch magnetoelastische Kopplung eine kohärente Phononenquelle zur Erzeugung hochfrequenter Spinwellen genutzt werden [25] und so die Lücke zwischen kohärenter Mikrowellenanregung und inkohärenter Erzeugung von Spinwellen durch fs-Laserpulse schließen [26].

Den phononischen Wärmetransport auf der Nanoskala zu verstehen, ist daher nicht nur allein von akademischen Interesse, sondern birgt das Potential für vielfältige Anwendungen in Technik und Wissenschaft. 


\subsection{Stand der Forschung}

Durch die herausragende Bedeutung von Wärmeenergie und ihrem Transport in allen Bereichen der Technik (Motoren, Thermoelektrika, Computerchips etc.) und des täglichen Lebens (Kochen, Heizen etc.) ist es nicht verwunderlich, dass auch die Erforschung der zugrunde liegenden Mechanismen des Wärmetransports einer der ältesten Bereiche der (Festkörper-)Physik ist. So stammt die erste empirische Beschreibung der Ausbreitung von Wärme in einem Festkörper von Joseph Fourier aus dem Jahr 1822. Das nach ihm benannte Fourier'sche Gesetz beschreibt den Wärmestrom $\dot{Q}$, welche in der Zeit $t$ zwischen einem heißen und einem kalten Punkt eines Festkörpers mit dem Temperaturgradienten $d T / d x$ über eine Fläche $A$ ausgetauscht wird [27]:

$$
\dot{Q}=-\kappa A \frac{\partial T}{\partial x} .
$$

Die materialabhängige Wärmeleitfähigkeit ist $\kappa$. Auf dem Fourier'schen Gesetz beruht auch die allgemeine Wärmeleitungsgleichung, welche die Form einer Diffusionsgleichung annimmt:

$$
\frac{\partial T(x, t)}{\partial t}=\frac{\kappa}{\rho c} \frac{\partial^{2} T(x, t)}{\partial x^{2}}
$$

$T(x, t)$ ist die Temperatur am Ort $x$ zur Zeit $t, \rho$ die Dichte des Mediums und $c$ die spezifische Wärmekapazität.

Mitte des 19. Jahrhunderts erkannten bedeutende Forscher wie James Joule [28], Robert Mayer [29] und William Thomson [1], dass die in einem Körper gespeicherte Wärme als (Gitter-)Schwingung der Atome vorliegt. Fast ein Jahrhundert später (1932) tauchte dann das erste mal der Begriff „Phonon“2 in dem Lehrbuch „Wave Mechanics: Elementary Theory" [30] von Jakow Frenkel auf, um den Teilchencharakter der elementaren Anregung der Gitterschwingungen zu bezeichnen. Die Annahme der im Gitter gespeicherten Wärme als Quasiteilchen, erlaubte es, die Wärmeleitfähigkeit auf das Spektrum der Phononen (also die Phononendispersion) und deren Streuraten zurückzuführen [31, 32]:

$$
\kappa(T)=k_{\mathrm{B}} \sum_{j} \int \frac{d^{3} q}{(2 \pi)^{3}} v_{j}(q)^{2} \tau_{j}(q) .
$$

\footnotetext{
${ }^{2}$ Eine Namensanalogie zu dem Quantenteilchen des Lichtfeldes „Photon“.
} 
Dabei geht die Summe über alle $j$-Schwingungszweige der des Phononenspektrums (näheres dazu in Kapitel 2.2.1) und das Integral über die Wellenvektoren $q$ der Phononen. $v_{j}(q)$ ist definiert als die Gruppengeschwindigkeit, also $v_{j}(q)=\partial \omega_{j} / \partial q_{j}$, und $\tau_{j}$ die Streurate der Phononen.

Mit der Anwendung der Boltzmann-Transport-Gleichung (BTE) auf den phononischen Wärmetransport [33-35] war dann auch eine hinreichend gute Beschreibung gefunden, welche unter Kenntnis von freier Weglänge und der Phononendispersion die Effekte von Korngrenzen, Grenzflächen und Defekten auf den Wärmetransport berücksichtigt hat.[36] Die Näherung der mittleren freien Weglänge über die reziproke Addition von empirisch bestimmten oder abgeschätzten Streuwahrscheinlichkeiten und Approximationen für die Phononendispersion, z.B. durch DebyeNäherung, hat sich für viele Vorhersagen und Experimente zur Wärmeleitung in unterschiedlichen Materialien als hinreichend erwiesen. So konnte beispielsweise ein mit klassischer Wärmediffusiongleichung nicht erklärbarer Heizeffekt am Übergang zwischen Drain- und Gate-Kontakt eines MOSFET mit der BTE durch Trennung der Phononen in einen akustischen und optischen Anteil erklärt werden. Der optische Anteil liefert mit kleiner Phononengeschwindigkeiten, im Gegensatz zum akustischen Anteil, nur einen kleinen Beitrag zum Wärmetransport. Der Hotspot tritt nun auf, weil die Elektronen am Kontaktübergang ihre Energie größtenteils an die optischen Phononenmoden abgeben. Die Wärmeenergie wird von dort nur langsam abtransportiert.[37, 38]

Mitte der Jahrtausendwende stieß die Entwicklung von Halbleiterchips, Moores Gesetz folgend, mit ihren Strukturen in die Größenordnung der mittleren freien Weglänge der Phononen vor. Es hat sich gezeigt, dass die Vorhersagen der BTE mit vereinfachten Parametern nicht mehr mit Ergebnissen aus Experimenten an Nanostrukturen übereinstimmten [18, 36, 39]. In den letzten zwei Dekaden hat sich daher ein großes Interesse um den nanoskopischen Wärmetransport entwickelt [40] und im Speziellen darum, unter welchen Bedingungen die Welleneigenschaften der Phononen berücksichtigt werden müssen [18, 36, 41-45]. Zur Untersuchung kommen eine ganze Reihe von Methoden und Modellsystemen zum Einsatz. Im Rahmen dieses Überblicks werde ich mich jedoch auf die für meine Arbeit wichtigen Multilagen- und Übergittersysteme, sowie auf die zeitabhängige Thermoreflektivität (TDTR) und die Transiente Thermoreflektivität (TTR) [46] beschränken. Für 
eine ausführlichere Übersicht zu Themen wie Wärmetransport in Nanopartikeln, Nanoröhren oder Graphen sei an dieser Stelle auf die Übersichtsartikel von Cahill et al. [36, 44] verwiesen.

Der Einfluss periodischer Nanostrukturen auf die Ausbreitung von Licht wurde bereits in den 80ern [47-49] beschrieben und erfolgreich [50, 51] zur Kontrolle von Lichtausbreitung in Nanostrukturen genutzt. Die Eigenschaften dieser sogenannten photonischen Kristalle sind heute gut beschrieben [52, 53].

In akustischen Übergittern (sogenannte phononische Kristalle) hat sich in frühen Messungen gezeigt, dass bei Reduzierung der Schichtdicke die Wärmeleitfähigkeit proportional zur Dichte der Grenzflächen abnimmt. In solchen Systemen kann der Wert von $\kappa$ durch den Beitrag der Grenzflächen auf die Streuung der Phononen unter dem Wert einer Legierung aus den Materialien liegen [54-58]. Im Jahr 2000 veröffentlichten Simkin und Mahan ihre theoretische Arbeit zum Wärmetransport in atomaren Übergittern [32]. In ihrer Veröffentlichung erweiterten sie die bisherige Beschreibung des phononischen Wärmetransportes um die emergenten Phononenzustände des Übergitters. Dadurch sind nun auch wellenmechanische Interferenzeffekte der Gitterschwingung in der BTE berücksichtigt. Ihre Berechnungen sagen einen kohärenten (ballistischen) Wärmetransport und ein Minimum der phononischen Wärmeleitfähigkeit voraus. Erst 12 Jahre später fanden Luckyanova et al. [59] einen ersten Hinweis auf diesen ballistischen Wärmetransport in GaAs/AlAs Übergittern: An diesen konnte gezeigt werden, dass die Wärmeleitfähigkeit sich unter $150 \mathrm{~K}$ linear zur Anzahl der Wiederholungen im Übergitter verhält und darüber konstant zur Anzahl der Wiederholung ist. Dies war ein indirekter Hinweis auf einen ballistischen Transport von Phononen in einem solchen Übergitter. Zwei Jahre später demonstrierten dann Ravichandran et al. [39] an Übergittern aus Strontiumtitanat und Kalziumtitanat den ersten experimentellen Nachweis des von Simkin und Mahan berechneten Minimums in der thermischen Leitfähigkeit. Damit erbrachten sie den ersten experimentellen Nachweis für kohärenten Wärmetransport in einem Übergitter. Molekulardynamik-Simulationen von $\mathrm{Li}$ et al. [60] sagen ein ähnliches Ergebnis für $\mathrm{SrTiO}_{3}$-Übergitter voraus. Ein Übergang von inkohärenten zu kohärenten Wärmetransport wurde ebenfalls in Metall/Halbleiter-Übergittern demonstriert [61, 62]. 
Nach meinem Kenntnisstand wurde seit der Veröffentlichung von Ravichandran et al. noch kein weiterer Versuch unternommen, die Beiträge von kohärentem und inkohärentem phononischen Wärmetransport in dielektrischen Übergittern anhand von experimentellen Messungen zu quantifizieren und mit dem gewonnenen Wissen die Eigenschaften des kohärenten Wärmetransportes zu beschreiben.

Dies liegt meiner Meinung nach an dem hohen Aufwand, Übergitter mit reproduzierbar hoher Qualität herzustellen [22] und dem ebenfalls hohen Aufwand, Wärmeleitfähigkeiten in komplexen Schichtstrukturen zu modellieren ${ }^{3}$ [18]. Mit der Metallorganischen Aerosoldeposition (MAD), dem Transienten Thermoreflektivitätsaufbau (TTR) und unserem numerischen Born-von-Kármán-Modell (BKModell) der Übergitter, konnten wir diese Herausforderungen jedoch meistern.

\subsection{Umfang dieser Arbeit}

Basierend auf vorangegangenen Arbeiten [63, 64], möchte ich in meiner Dissertation zunächst demonstrieren, wie eine Oberflächenmode in einer Probe, bestehend aus einer Multilage und einer Resonatorschicht, angeregt werden kann. Die Frequenz der Oszillation in der Resonatorschicht fällt in die akustische Bandlücke der Multilage. Die Anregung dieser Mode ist daher in der Multilage selbst verboten. Variation der Resonatorschichtdicke erlaubt darüber hinaus Rückschlüsse auf die akustische Bandlücke der Multilage.

Dieses Beispiel demonstriert das Blockieren einer kohärenten akustischen Mode durch den Einsatz einer strukturierten Multilage (engl. coherent phonon blocking). Im nächsten Schritt werden wir die Probensysteme zu höheren Frequenzen skalieren und untersuchen, ob wir das Blockieren kohärenter Phononen auch in den stark korrelierten $\mathrm{LaMnO}_{3} / \mathrm{SrMnO}_{3}-$ Manganat-Übergittern wiederfinden und welchen Einfluss dies auf den thermischen Transport in den Übergittern hat.

Um besagten Themen nachzugehen, werden zunächst die allgemeinen mathematische und physikalischen Grundlagen von elastischen Schallwellen in dünnen Schichten vorgestellt. Wir bleiben dabei gänzlich im Rahmen der klassischen kontinuums-

${ }^{3}$ Das Modell von Simkin und Mahan eignet sich beispielsweise nur für eine qualitative Beschreibung des kohärenten Wärmetransports. Die Annahme einer konstanten freien Weglänge im Modell erscheint eine zu einfache Annahme zu sein. 
mechanischen Elastizitätstheorie. Erst mit dem Übergang von Schall zu den hochfrequenten Gitterschwingungen, den Phononen, verlassen wir teilweise den klassischen Bereich der Physik. Die Theorie möchte ich dann mit einer Beschreibung des Wärmetransportes unter Einbeziehung der dreidimensionalen Dispersionsrelation der Phononen in einem Übergitter abschließen.

Experimentell wird diese Arbeit einem ähnlichen Verlauf folgen: Zunächst werde ich bei (vergleichsweise) niedrigen Frequenzen beginnen und die Dispersionsrelation von GHz-Ultraschallschwingungen in Multilagensystemen aus $\mathrm{ZrO}_{2}$ und $\mathrm{MgO}$ untersuchen. Die Messungen hierzu erfolgten durch Ultrakurzzeitspektroskopie.

Anschließend werden die gewonnenen Erkenntnisse aus den Multilagenschichten durch ein neues Probensystem zu höheren Frequenzen skaliert. Mit Hilfe der MAD konnte die Gruppe um Prof. Dr. Moshnyaga epitaktische Strontiummanganat/Lanthanmaganat $\left(\mathrm{LaMnO}_{3}\right.$ (LMO) und $\mathrm{SrMnO}_{3}$ (SMO)) Übergitter mit atomarer Präzision für unser gemeinsames Projekt ${ }^{4}$ im Sonderforschungsbereich 1073 herstellen. Durch das Herunterskalieren der Schichtdicken um etwa den Faktor 100 werden auch die durch das Übergitter beeinflussten Schwingungsfrequenzen vom GHz- in den THz-Bereich verschoben. In diesem Bereich adressieren wir direkt die für den Wärmetransport verantwortlichen Phononenmoden [65, 66]. Hier stoßen wir mit der Ultrakurzzeitspektroskopie jedoch an eine Auflösungsgrenze: Die Eindringtiefe von Laserlicht mit $\lambda=800 \mathrm{~nm}$ in z.B. Kupfer ist etwa $13 \mathrm{~nm}$ [67]. Die Wellenlänge eines Phonons mit einer Frequenz von $1 \mathrm{THz}$ und einer Gruppengeschwindigkeit von $5000 \mathrm{~m} \mathrm{~s}^{-1}$ wäre $5 \mathrm{~nm}$, also kleiner als der Bereich, aus dem das Reflexionssignal stammt. Bei kohärenten Phononenmoden in der gesamten Dicke der Schicht kommt hinzu, dass sich Signalamplituden über dem Messbereich hinweg mitteln würden.

Aus diesem Grund habe ich mit einem eigens für diesen Zweck aufgebauten Thermoreflektivitätsexperiment den Wärmetransport direkt untersucht und so die Wärmeleitfähigkeiten verschiedener Übergitter bestimmt.

Die experimentellen Methoden, die Auswertungsmethoden und die Probenherstellung werde ich im Kapitel Experimentelle Methoden ausführlich schildern.

\footnotetext{
${ }^{4}$ SFB 1073 Teilprojekt A02.
} 
Begleitet wurden die Experimente stets durch eine ausführliche analytische oder numerische Untersuchung. Dies dient dazu, die Messergebnisse zu interpretieren und aus ihnen neue Experimente abzuleiten.

Abschließend werde ich alle Ergebnisse noch einmal kurz zusammenfassen und einen Ausblick auf mögliche weiterführende Experimente geben sowie denkbare Anwendungen der gewonnen Erkenntnisse vorstellen. Zu letzterem gehört unter anderem die Idee, einen optisch gepumpten, akustischen Oszillator aus unseren akustischen Multilagen herzustellen. Eine mögliche Anwendung dieses Systems wäre zum Beispiel die Erzeugung hochfrequenter Spinwellen für den Einsatz in magnonischen Schaltkreisen. Ich werde hierzu vorbereitende Simulationen und Berechnungen präsentieren. Des Weiteren werde ich im Ausblick Berechnungen zu einem Phononenisolator mit einer theoretischen Wärmeleitfähigkeit von $0.01 \mathrm{~W} \mathrm{~m}^{-1} \mathrm{~K}^{-1}$ vorstellen. Eine experimentelle Demonstration von Phononenisolator und PhononenSpinwellen-Resonator war aufgrund der unerwarteten Pandemie im Frühjahr 2020 jedoch leider nicht mehr möglich.

An dieser Stelle sei noch kurz erwähnt, dass ich auf ein eigenständiges Kapitel Diskussion verzichte und Ergebnisse direkt an Ort und Stelle in den Gesamtkontext einordnen werde. 


\section{Kapitel 2}

\section{Theorie}

In diesem Kapitel möchte ich sowohl die mathematischen Grundlagen als auch die physikalischen Zusammenhänge, die für die spätere Interpretation der Ergebnisse wichtig sind, in einer nachvollziehbaren Weise darstellen.

Ich beginne zunächst mit einer allgemeinen Einführung in mechanischen Schwingungen, genauer gesagt dem akustischen Schall in einem Volumenmaterial. Im gleichen Zuge werde ich die Schalldispersion in einem Multilagensystem herleiten. Anschließend wende ich mich den mikroskopischen Gitterschwingungen des Festkörpers, den Phononen, zu. Hierzu führe ich zunächst ein eindimensionales FederMasse-Modell und darauf aufbauend ein dreidimensionales Born-von-KármánModell kristalliner Festkörper ein. Mit Hilfe dieses Modelles werde ich anschließend einen Weg aufzeigen, die phononische Wärmeleitfähigkeit anhand der Phononenbandstruktur von komplexen Übergittern zu berechnen.

\subsection{GHz- und THz-Gitterschwingungen}

Bei der Betrachtung von Proben aus sich periodisch wiederholenden Schichten mit einer Schichtdicke $d$ deutlich größer als die Gitterkonstanten $c$, also $d \gg c$, sprechen wir im Rahmen dieser Arbeit von Multilagen. Sind die Einzelschichtdicke hingegen im Bereich der Gitterkonstanten $d \geq c$, sprechen wir von Übergittern (ÜG). Bei typischen Schallgeschwindigkeiten von $v_{L}=1000-10000 \mathrm{~m} \mathrm{~s}^{-1}$ und Schichtdicken im Nanometerbereich beobachten wir in diesen Multilagen senkrecht zur Stapelrichtung charakteristische Frequenzen von $f=\frac{v_{L}}{d} \approx 1-1000 \mathrm{GHz}$ [68]. 
Für weitere Betrachtungen der Dynamik in Multilagen ist die Dispersion der akustischen Wellen, also der Zusammenhang von Frequenz und Wellenvektor der Schallwellen, von großer Bedeutung. Die Dispersion für eine longitudinale Schallwelle in einem isotropen Festkörper in eine der Symmetrierichtungen $z$ mit den Auslenkungen $u_{z}(z, t)$ möchte ich im Folgenden herleiten. Für nicht isotrope Kristallgitter, transversale Schallwellen oder Raumrichtungen abseits der drei Symmetrieachsen ist die Herleitung der Dispersion ähnlich, aber in Hinblick auf meine späteren Experimente an Multilagen aus dünnen Schichten, in welchen wir die Ultraschallausbreitung im Wesentlichen als eindimensional betrachten können, an dieser Stelle wenig instruktiv.

Schauen wir uns nun zunächst ein kleines Volumen dieses Festkörpers an. Die Kantenlänge des betrachteten Volumens sei in allen drei Raumdimensionen $l$. Wirkt nun an der xy-Fläche bei $z$ eine Spannung $\sigma_{z z}(z)$ in z-Richtung und an der gegenüberliegenden Seite bei $z+l$ eine Spannung $\sigma_{z z}(z+l)$, können wir mit Hilfe des Hook'schen Gesetz $F=\sigma \cdot l^{2}$ mit $\sigma_{i j}=\sum_{k l} c_{i j k l} e_{k l}, e_{k l}=\frac{\partial u_{k}}{\partial l}$ und den Einträgen des Elastizitätstensors $c_{i j k l}$ die wirkende Kraft in z-Richtung als $F_{z}=\left(\sigma_{z z}(z+l)-\sigma_{z z}(z)\right) \cdot l^{2}$ schreiben. Für die Betrachtung von longitudinalen Wellen in einem isotropen Festkörper in $z$-Richtung ist die eindimensionale Betrachtung ausreichend und mit infinitesimal kleinen Kanten $l$ geht dies über zu

$$
F_{z}=\frac{\partial \sigma_{z z}(z)}{\partial z} l^{3}
$$

Mit dem 2. Newtonschen Gesetz, der Dichte $\rho$ und $a_{z}=\frac{\partial^{2} u_{z}(z)}{\partial t^{2}}$ können wir dies schreiben als :

$$
\begin{array}{r}
F_{z}=m \cdot a_{z}=\rho l^{3} \frac{\partial^{2} u_{z}(z)}{\partial t^{2}}=\frac{\partial \sigma_{z z}(z)}{\partial z} l^{3} \\
\Rightarrow \rho \frac{\partial^{2} u_{z}(z)}{\partial t^{2}}=\frac{\partial \sigma_{z z}(z)}{\partial z} \\
\text { mit } \sigma_{z z}(z)=c_{11} \frac{\partial u_{z}(z)}{\partial z} \\
\Rightarrow \rho \frac{\partial^{2} u_{z}(z)}{\partial t^{2}}=\frac{\partial}{\partial z}\left(c_{11} \frac{\partial u_{z}(z)}{\partial z}\right) \\
\Rightarrow \rho \frac{\partial^{2} u_{z}(z)}{\partial t^{2}}=c_{11} \frac{\partial^{2} u_{z}(z)}{\partial z^{2}}
\end{array}
$$


Mit dem Ansatz ebener Wellen $u(z, t)=u_{0} e^{i(q x-\omega t)}$ mit der Frequenz $\omega$ und der Wellenzahl $q$ ergibt sich die Dispersionsrelation longitudinaler Schallwellen zu:

$$
\omega=\sqrt{\frac{c_{11}}{\rho}} q=v_{l} q
$$

$v_{l}=\sqrt{\frac{c_{11}}{\rho}}=\frac{\omega}{q}$ ist die longitudinale Schallgeschwindigkeit.

Nun wollen wir uns den Fall einer idealisierten Multilagenprobe mit unendlich vielen periodischen Wiederholungen der Doppellagen aus zwei verschiedenen Materialien mit den Konstanten $v_{1}, v_{2}, \rho_{1}, \rho_{2}$ und den jeweiligen Schichtdicken $d_{1}$ und $d_{2}$ anschauen. Für die longitudinale Auslenkung in z-Richtung $u_{z}(z)=u_{n, 1}(z)$ in der ersten Schicht der $n$-ten Doppellage wählen wir einen Blochansatz, gemäß

$$
u_{\mathrm{n}, 1}(z)=e^{i q n d}\left(A e^{i \alpha_{1}(z-n d)}+B e^{-i \alpha_{1}(z-n d)}\right) \text { für } z \in\left[n d, n d+d_{1}\right]
$$

und für die Auslenkung $u_{n, 2}(z)$ in der zweiten Schicht

$$
u_{\mathrm{n}, 2}(z)=e^{i q n d}\left(C e^{\left.i \alpha_{2}\left(z-n d-d_{1}\right)\right)}+D e^{-i \alpha_{2}\left(z-n d-d_{1}\right)}\right) \text { für } z \in\left[n d+d_{1},(n+1) d\right] .
$$

$e^{i q n d}$ ist ein gitterperiodischer Phasenfaktor.

An den Grenzflächen müssen Auslenkung und mechanische Spannung stetig sein, weshalb wir als Randbedingungen

$$
\begin{aligned}
& u_{n, 1}(n d)=u_{(n-1), 2}(n d) \\
& u_{n, 1}\left(n d+d_{1}\right)=u_{n, 2}\left(n d+d_{1}\right)
\end{aligned}
$$

und mit der elastischen Spannung $\sigma_{n, i}(z)=c_{11} \frac{\partial}{\partial z} u_{n, i}(z)$

$$
\begin{aligned}
& \sigma_{n, 1}(n d)=\sigma_{(n-1), 2}(n d) \\
& \sigma_{n, 1}\left(n d+d_{1}\right)=\sigma_{n, 2}\left(n d+d_{1}\right)
\end{aligned}
$$


wählen. Damit erhalten wir das Gleichungssystem

$$
\begin{array}{r}
A+B-C e^{\beta_{2}} e^{-i q d}-D e^{-\beta_{2}} e^{-i Q d}=0 \\
A e^{\beta_{1}}+B e^{-\beta_{1}}-C-D=0 \\
A \gamma_{1}-B \gamma_{1}-C \gamma_{2} e^{\beta_{2}} e^{-i Q d}+D \gamma_{2} e^{-\beta_{2}} e^{-i q d}=0 \\
A \gamma_{1} e^{\beta_{1}}-B \gamma_{1} e^{-\beta_{1}}-C \gamma_{2}+D \gamma_{2}=0
\end{array}
$$

mit $\beta_{i}=i \alpha_{i} d_{i}$ und $\gamma_{i}=\alpha_{i} \rho_{i} v_{i}^{2}$. Als Matrix lässt sich dieses System in Form einer $4 \times 4$-Matrix schreiben:

$$
M=\left[\begin{array}{cccc}
1 & 1 & -e^{\beta_{2}} e^{-i q d} & -e^{-\beta_{2}} e^{-i q d} \\
e^{\beta_{1}} & e^{-\beta_{1}} & -1 & -1 \\
\gamma_{1} & -\gamma_{1} & -\gamma_{2} e^{\beta_{2}} e^{-i q d} & \gamma_{2} e^{-\beta_{2}} e^{-i q d} \\
\gamma_{1} e^{\beta_{1}} & -\gamma_{1} e^{-\beta_{1}} & -\gamma_{2} & \gamma_{2}
\end{array}\right]
$$

Die Lösung des Gleichungssystem folgt über $\operatorname{det}(M)=0$. $\operatorname{det}(M)$ wird mit Hilfe des Laplaceschen Entwicklungssatzes berechnet (siehe Anhang A.2). Sie lautet:

$$
\cos \left(\frac{\omega d_{1}}{v_{1}}\right) \cos \left(\frac{\omega d_{2}}{v_{2}}\right)-\frac{1}{2}\left(\frac{\rho_{1} v_{1}}{\rho_{2} v_{2}}+\frac{\rho_{2} v_{2}}{\rho_{1} v_{1}}\right) \cdot \sin \left(\frac{\omega d_{1}}{v_{1}}\right) \cdot \sin \left(\frac{\omega d_{2}}{v_{2}}\right)=\cos (q d)
$$

Gleichung (2.10), auch bekannt als Rytov-Gleichung [69, 70], beschreibt implizit die 1D-Dispersion $\omega(q)$ longitudinaler Schallwellen in einem unendlich ausgedehnten Schichtstapel aus Doppellagen senkrecht zur Stapelrichtung.

Zur Klassifizierung führen wir nun die Impedanzfehlanpassung $\chi=\frac{1}{2}\left(\frac{Z_{1}}{Z_{2}}+\frac{Z_{2}}{Z_{1}}\right)$ mit der materialspezifischen, akustischen Impedanz $Z_{i}=\rho_{i} v_{i}$ ein. Den Einfluss von $\chi$ auf die Dispersion gemäß Gl. (2.10) will ich nun an einigen Beispielen zeigen:

Schauen wir uns zunächst den Fall an, wenn die beiden Schichten unserer Multilage die selbe Dichte und Schallgeschwindigkeit besitzen, also $\rho_{1}=\rho_{2}$ und $v_{1}=v_{2}$. In diesem Fall ist $\chi=1$ und nimmt den kleinsten möglichen Wert an. Bei einer Schichtdicke von $d_{1}=d_{2}=10 \mathrm{~nm}$ erhalten wir die Dispersion, wie sie in Abbildung 2.1 dargestellt ist. Dabei trennen wir die Lösung von $q$ in Real- und Imaginärteil $q=Q^{\prime}+i \cdot Q^{\prime \prime}$ auf. Wie erwartet erhalten wir als Lösung des Realteils eine lineare Dispersion mit einer Steigung von $\partial \omega / \partial q=5000 \mathrm{~ms}^{-1}$. $Q^{\prime \prime}$ ist durchgängig null. 


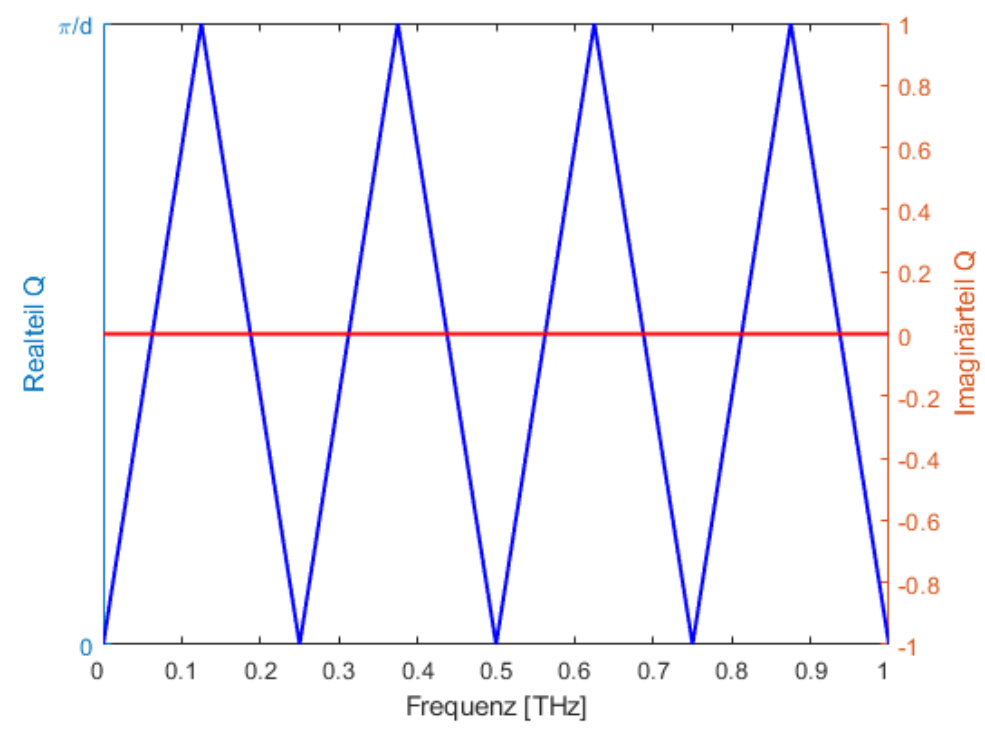

Abbildung 2.1: Einfache Dispersion einer Multilage mit identischen Materialparametern. $v_{1}=v_{2}=5000 \mathrm{~m} \mathrm{~s}^{-1}$ ist so gewählt, dass es im Bereich typischer Metalle oder Oxide liegt.

Nun wählen wir Materialparameter mit einer Fehlanpassung von $\chi=1.25$, indem wir die Dichte der zweiten Schicht verdoppeln $2 \rho_{1}=\rho_{2}$.

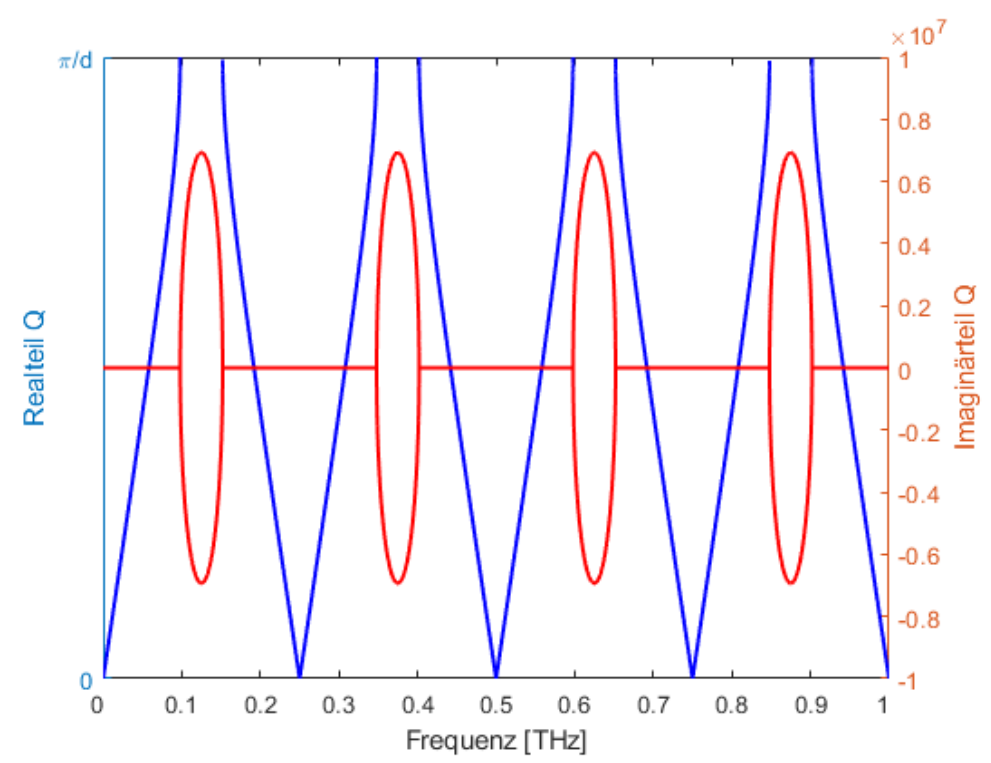

Abbildung 2.2: Dispersion einer Multilage mit Materialparametern wie in Abbildung 2.1 , jedoch mit $2 \rho_{1}=\rho_{2}$. 
Wir sehen, dass sich am Rand der ersten Brillouinzone Bandlücken öffnen, in welchen der Realteil $Q^{\prime}$ verschwindet und die Lösung der Rytovgleichung rein imaginär ist. Die Lösung in der Bandlücke mit $Q^{\prime \prime}>0$ ist eine evaneszente Schwingungsmode, die beim Durchqueren unserer Multilage exponentiell abklingt.

Nun betrachten wir noch den Fall, dass $\chi=1.25, \rho_{1}=\rho_{2}$ aber $2 \cdot v_{1}=v_{2}$ :

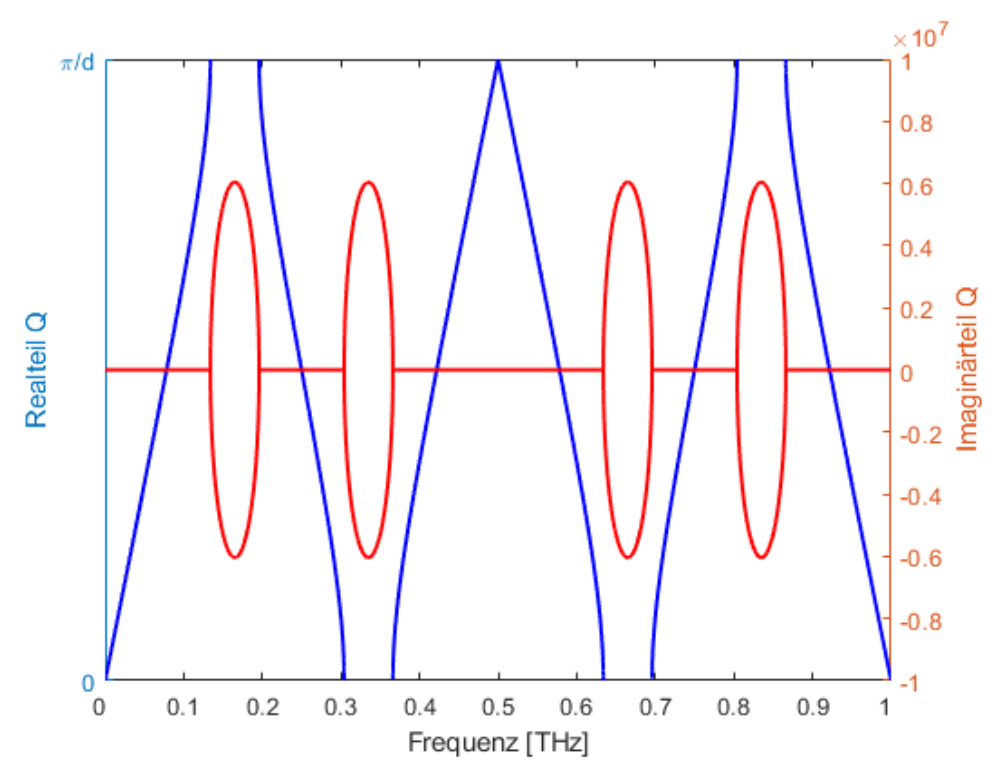

Abbildung 2.3: Dispersion einer Multilage mit den Materialparametern wie in Abbildung 2.1, jedoch mit $2 v_{1}=v_{2}$.

In diesem Fall tun sich am Rand und in der Mitte der Brillouinzone Bandlücken auf.

Als ein realistisches Beispiel sollen uns nun eine Multilage dienen, welche mit den Materialparametern von Magnesiumoxid $(\mathrm{MgO})$ und Zirkondioxid $\left(\mathrm{ZrO}_{2}\right)$ berechnet wurde. In Abb. 2.4 sehen wir die akustische Dispersion einer solchen Multilage. 


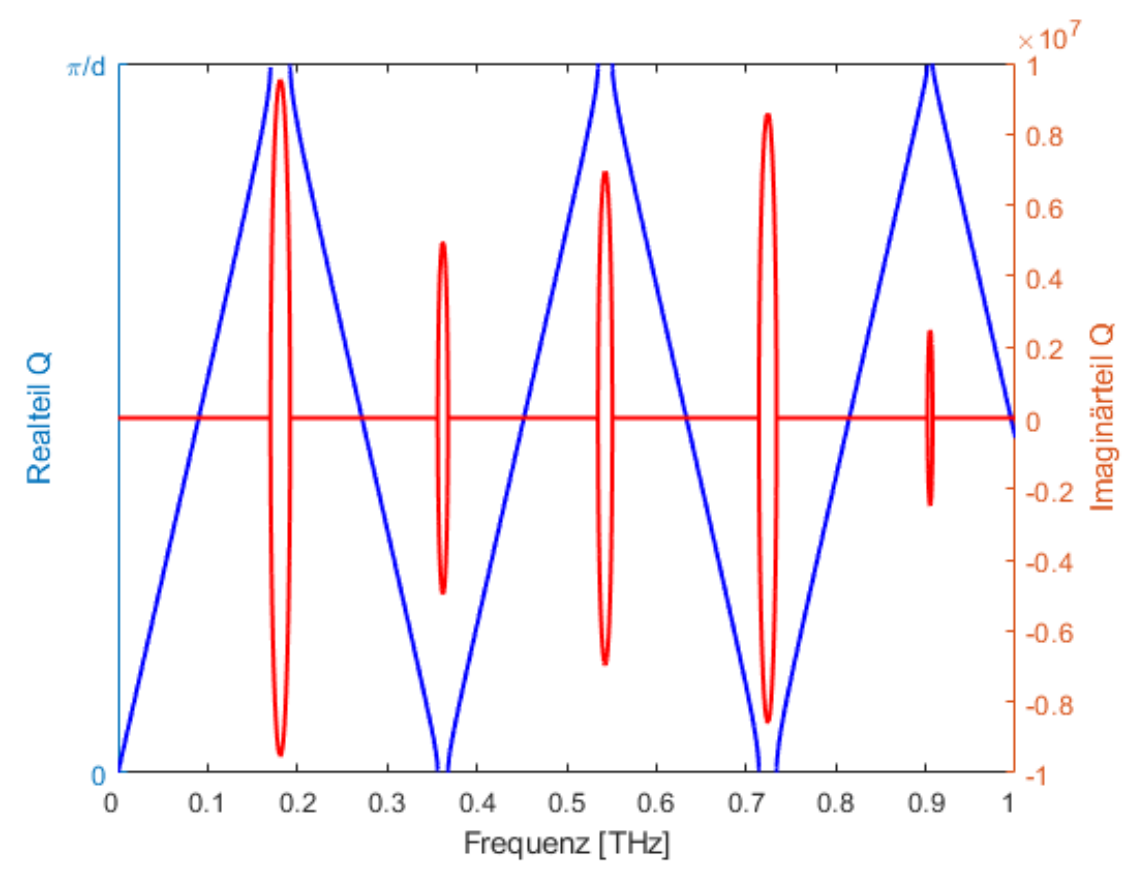

Abbildung 2.4: Dispersion einer Multilage mit den Materialparametern $\rho_{\mathrm{MgO}}=$ $3580 \mathrm{~kg} \mathrm{~m}^{-3}, \rho_{\mathrm{ZrO}_{2}}=6120 \mathrm{~kg} \mathrm{~m}^{-3}, v_{\mathrm{MgO}}=8700 \mathrm{~m} \mathrm{~s}^{-1}$ und $v_{\mathrm{ZrO}_{2}}=6200 \mathrm{~m} \mathrm{~s}^{-1}$.

Die Bandlücken fallen hier deutlich schmaler als in unseren künstlichen Beispielen aus. Das ist jedoch erwartet, da diese Materialkombination nur eine kleine akustische Fehlanpassung von etwa $\chi=1.02$ hat. Wie wir jedoch später im experimentellen Teil (Kapitel 4.1.1) sehen werden, ist diese kleine Fehlanpassung bereits ausreichend um die erste Bandlücke einer solchen Multilage im Experiment beobachten zu können.

Aus praktischen Gründen werden solche Multilagen meist mit einer Deckschicht versehen, die entweder als Schutz oder als Ort der Anregung dient z.B. in einem Pump-Probe-Experiment mittels Laseranregung. Wir erweitern unser Modell aus den Gleichungen (2.4) und (2.5) mit einer dritten, einzelnen Schicht der Dicke $d_{\text {Cap. }}$ und der zugehörigen mechanischen Auslenkung $d_{\text {Cap. }}(z)$ :

$$
u_{\text {Cap. }}(z)=E e^{i \alpha_{\text {Cap. }} z}+F e^{-i \alpha_{\text {Cap. }} z} \quad \text { für } z \in\left[-d_{\text {Cap. }}, 0\right]
$$

Hier ist analog $\alpha_{\text {Cap. }}=\frac{\omega}{v_{\text {Cap. }}}$. Zusätzlich zu den oben genannten Randbedingungen von versatzfreien Grenzflächen und kontinuierlicher, mechanischer Spannung 
setzen wir für die Oberfläche der Probe noch Spannungsfreiheit voraus. Die zusätzlichen Randbedingungen sind somit

$$
\begin{aligned}
& u_{\text {Cap. }}(0)=u_{0,1}(0) \\
& \sigma_{\text {Cap. }}(0)=\sigma_{0,1}(0) \\
& \sigma_{\text {Cap. }}\left(-d_{\text {Cap. }}\right)=0 .
\end{aligned}
$$

Das Gleichungssystem nimmt also die Form

$$
\begin{aligned}
A+B-C e^{\beta_{2}} e^{-i Q d}-D e^{-\beta_{2}} e^{-i Q d} & =0, \\
A e^{\beta_{1}}+B e^{-\beta_{1}}-C-D & =0, \\
A \gamma_{1}-B \gamma_{1}-C \gamma_{2} e^{\beta_{2}} e^{-i Q d}+D \gamma_{2} e^{-\beta_{2}} e^{-i Q d} & =0, \\
A \gamma_{1} e^{\beta_{1}}-B \gamma_{1} e^{-\beta_{1}}-C \gamma_{2}+D \gamma_{2} & =0, \\
0 \cdot A+0 \cdot B+0 \cdot C+0 \cdot D+E e^{-\beta_{\text {Cap. }}}-F e^{+\beta_{\text {Cap. }}} & =0, \\
A+B+0 \cdot C+0 \cdot D-E-F & =0, \\
\gamma_{1} A-\gamma_{1} B+0 \cdot C+0 \cdot D-\gamma_{\text {Cap. }} E+\gamma_{\text {Cap. }} F & =0
\end{aligned}
$$

an. Da wir nun sieben Gleichungen und sechs freie Parameter haben, kombinieren wir zunächst Zeile 5 und 6 in Gleichung (2.13) zu:

$$
-A-B+0 \cdot C+0 \cdot D+E\left(e^{-\beta_{\text {Cap. }}}+1\right)-F\left(e^{+\beta_{\text {Cap. }}}-1\right)=0 .
$$

Das Gleichungssystem lässt sich analog zur Rytov-Gleichung über Bestimmung der Determinante lösen. Aufgrund der sechs Unbekannten A-F wird die Determinante sehr unhandlich. Für unsere Zwecke ist es jedoch ausreichend, das Gleichungssystem über einen numerischen Solver in z.B. Matlab oder Scilab für explizite Materialparameter zu lösen. 


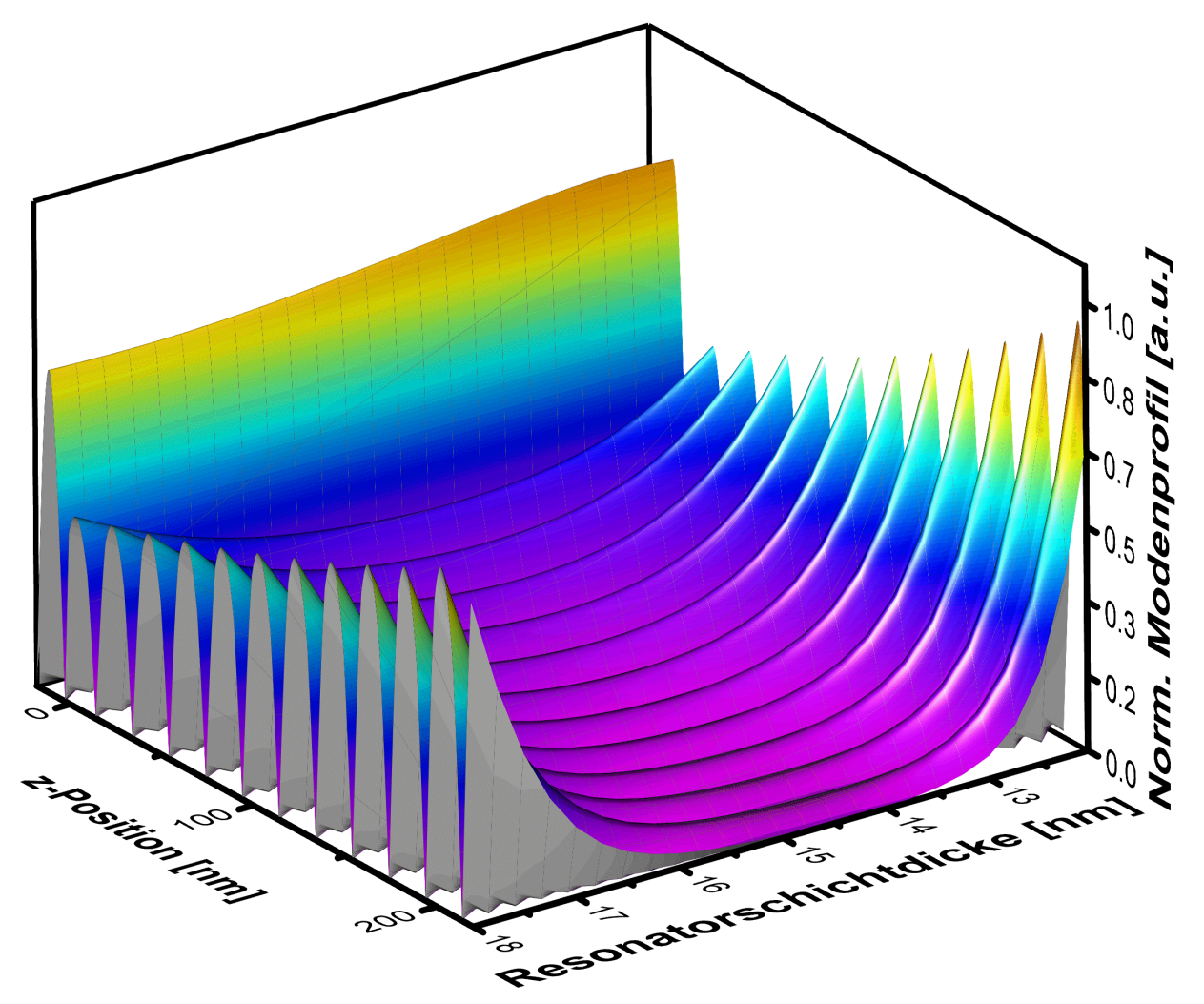

Abbildung 2.5: Modenprofil des gekoppelten Systems aus Deckschicht und Übergitter als Funktion der Position im Schichtstapel und der Deckschichtdicke.

Die so berechnete Dispersionsrelation $\omega(q)$ ist im Bereich der Multilage identisch mit der zuvor berechneten Lösung der unendlich ausgedehnten Multilage. Mit dieser numerischen Lösung lässt sich jedoch zusätzlich das Modenprofil in der Deckschicht berechnen. In Abb. 2.5 ist ein berechnetes Modenprofil der maximalen elastischen Verspannung für einen Stapel aus $\mathrm{MgO} / \mathrm{ZrO}_{2}$ mit $10 \mathrm{~nm}$ Schichtdicke und mit einer in ihrer Dicke variablen Wolframdeckschicht (Resonatorschichtdicke in Abb. 2.5) dargestellt. Der Nullpunkt auf der Positionsachse ist die Grenzfläche zwischen Wolfram und Multilage. Es ist gut zu erkennen, dass die maximale Spannungsamplitude in der Deckschicht fast unabhängig von der Schichtdicke ist. 
In der Multilage zeigt das Modenprofil eine deutlich größere Abhängigkeit von der Wolframschichtdicke. Bei dicken und dünnen Wolframschichten von $12 \mathrm{~nm}$ und $18 \mathrm{~nm}$ steigt die Amplitude mit jeder weiteren Schicht bis ins Unendliche an. Es ist offensichtlich, allein schon aus Energieerhaltungsgründen, dass dies eine unphysikalische Lösung des Gleichungssystems ist. Eine realistische Annahme wäre jedoch, dass Schwingungsmoden mit diesen Frequenzen die Multilage ungedämpft durchqueren können. Dies sollte folglich zu einem Abfließen der Schwingungsenergie und damit auch zu einer Dämpfung der Wolframeigenmode führen. Im Bereich von $13 \mathrm{~nm}$ bis $17 \mathrm{~nm}$ Wolframdicke haben wir den umgekehrten Fall: Die maximale Schwingungsamplitude nimmt exponentiell mit jeder weiteren Schicht der Multilage ab - die Schwingungsenergie kann das Wolfram nicht über die Multilage verlassen. Der Unterschied der Moden für Wolframdicken von $12.0 \mathrm{~nm}$ und $14.5 \mathrm{~nm}$ ist noch einmal in Abb. 2.6 dargestellt.

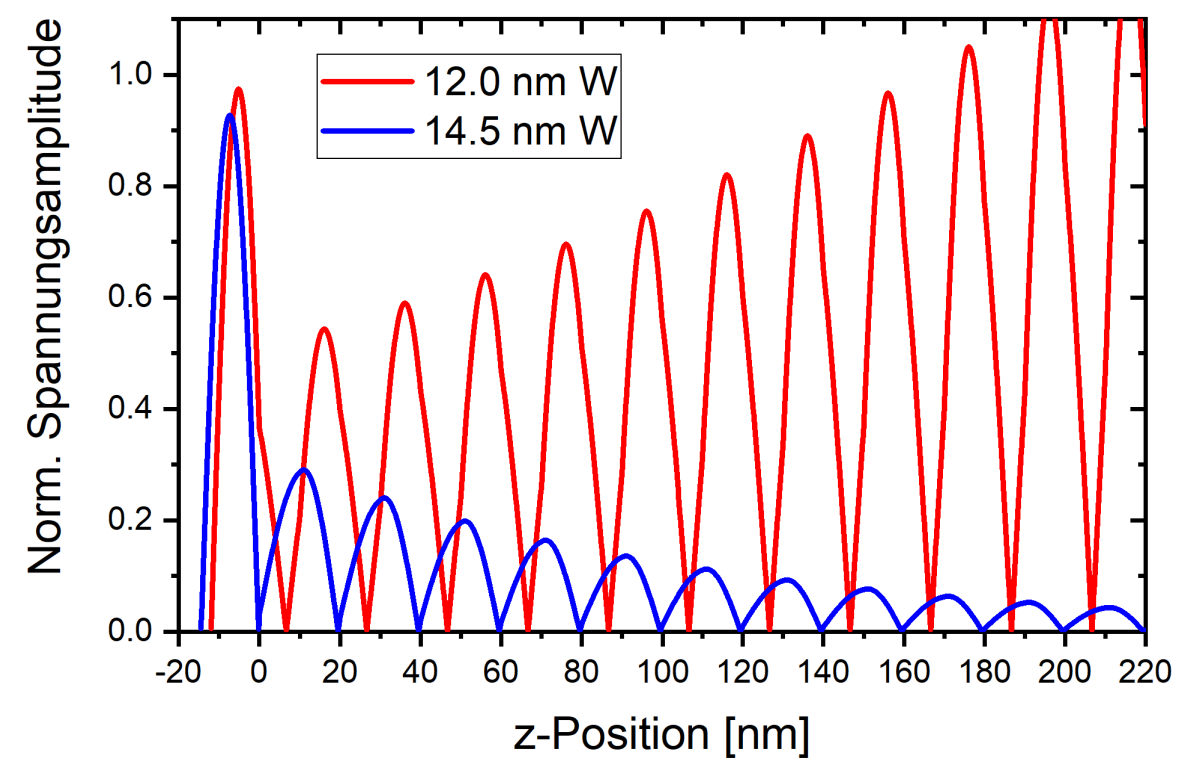

Abbildung 2.6: Modenprofil für die Wolframdeckschichtdicken $12 \mathrm{~nm}$ (rot) und $14.5 \mathrm{~nm}$ (blau). Multilage berechnet mit $\rho_{\mathrm{MgO}}=3580 \mathrm{~kg} \mathrm{~m}^{-3}, \rho_{\mathrm{ZrO}_{2}}=6120 \mathrm{~kg} \mathrm{~m}^{-3}$, $v_{\mathrm{MgO}}=8700 \mathrm{~m} \mathrm{~s}^{-1}$ und $v_{\mathrm{ZrO}_{2}}=6200 \mathrm{~m} \mathrm{~s}^{-1}$.

Die x-Achsenbeschriftung ist so skaliert, dass die Grenzflächen der Multilage an den großen Hilfsstrichen liegen. Es ist gut zu erkennen, dass die Mode in der Probe mit $d_{W}=12.0 \mathrm{~nm}$ (rot) die Schwingungsknoten nicht an den Grenzflächen hat. Die 
Eigenmode der Wolframschicht mit $d_{W}=14.5 \mathrm{~nm}$ (blau) jedoch passt zu den Wiederholungen der Multilagengrenzflächen. Die Eigenfrequenz einer freistehenden Wolframschicht mit dieser Dicke wäre etwa bei $f_{\mathrm{W}}=\frac{v_{\mathrm{W}}}{2 \cdot d_{\mathrm{W}}}=\frac{5220 \mathrm{~ms}^{-1}}{29 \mathrm{~nm}}=180 \mathrm{GHz}$. Erinnern wir uns an die Dispersionsrelation der $\mathrm{MgO} / \mathrm{ZrO}_{2}$ Multilage mit $20 \mathrm{~nm}$ Doppellagen (Abb. 2.2). Dann sehen wir, dass die Mitte der ersten Bandlücke ebenfalls genau bei $f_{\mathrm{BG} 1}=180 \mathrm{GHz}$ liegt. In unserem Modellsystem haben wir also genau bei $d_{W}=14.5 \mathrm{~nm}$ Wolframdicke eine Übereinstimmung der Eigenfrequenz des Wolframfilms und der zentralen Frequenz der ersten Bandlücke der Multilage. In Analogie zur Optik können wir dieses System als einen akustischen Oszillator betrachten, in dem die Anregung einer abgestimmten Frequenz (Eigenfrequenz Wolframschicht $=$ Frequenz in Bandlücke der Multilage) zwischen einem BraggSpiegel und der akustisch reflektierenden Probenoberfläche eingeschlossen ist. Die hier berechnete Probenstruktur und drei exemplarische Modenprofile mit der Probenstruktur im Hintergrund sind noch einmal in nachfolgender Grafik skizziert:

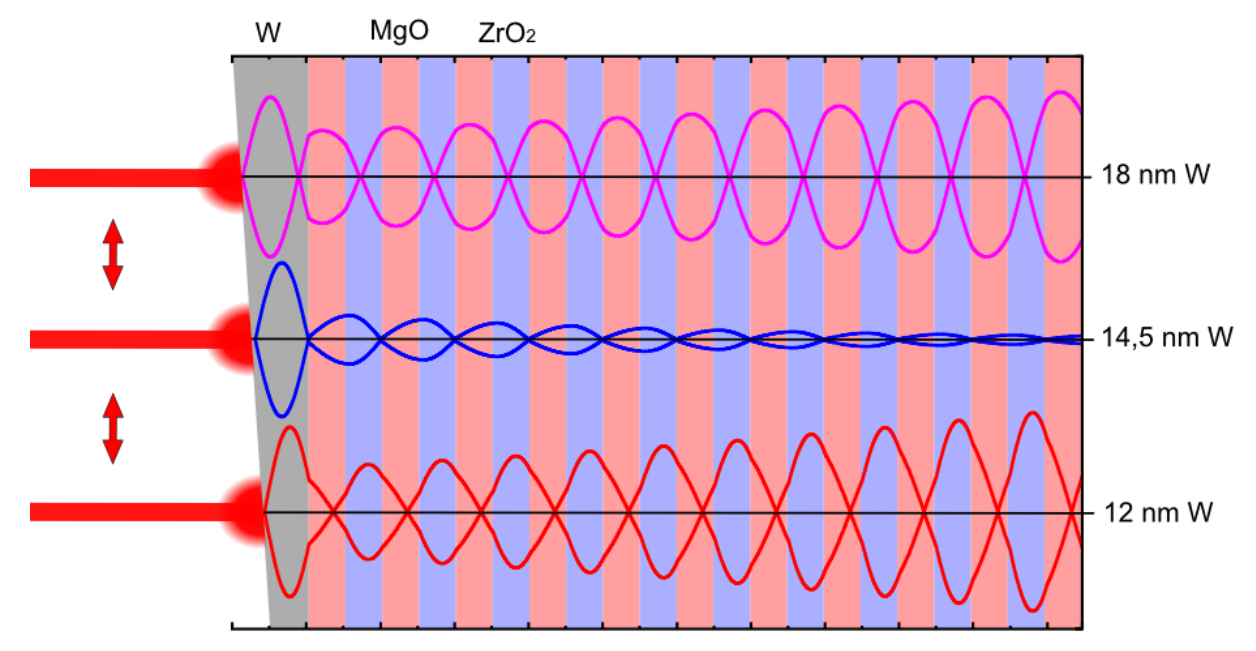

Abbildung 2.7: Modelprofil für die Wolframdeckschichtdicken $18 \mathrm{~nm}$ (rosa) und $14.5 \mathrm{~nm}$ (blau) und $12 \mathrm{~nm}$ (rot). Im Hintergrund ist der Aufbau der berechneten Struktur aus $\mathrm{MgO} / \mathrm{ZrO}_{2}$ Übergitter mit in der Dicke variabler Wolframschicht an der z.B. eine Anregung der Wolframeigenmode per Laserpuls stattfindet. 


\subsection{Phononen}

Bereits in der Mitte des 19. Jhd. stellten Forscher wie William Thomson, Baron von Kelvin fest ${ }^{1}$, dass die Wärme in einem (nicht elektrisch leitfähigen) Festkörper in Form von mechanischer Energie, also in Gitterschwingungen, gespeichert ist [1]. Im vorangegangen Kapitel 2.1 haben wir uns bereits die akustischen Schwingungsmoden in einem Schichtstapel aus vergleichsweise dicken Schichten von mehreren Nanometern angeschaut und die Dispersionsrelation berechnet, also den Zusammenhang zwischen Frequenz und Wellenvektor dieser Schwingungen. In diesem Kapitel wollen wir uns nun die thermisch angeregten Gitterschwingungen eines Festkörperkristalls anschauen. Genau wie die zueinander schwingenden Schichten in unseren Multilagen haben auch die gegeneinander schwingenden Atome in einem Festkörper diskrete Schwingungsmoden. Die Anregung einer dieser Normalschwingungen, also ein Anregungsquant des Gitters, nennen wir ein Phonon [30]. Im Welle-Teilchen-Dualismus können wir diese Schwingungsanregung des Gitters als bosonische Quasiteilchen interpretieren. Das ermöglicht die Einführung von bekannten Konzepten wie mittlere (Teilchen-)Geschwindigkeit, mittlere freie Weglänge und Streuwahrscheinlichkeiten für Phononen, welche eine relative einfache Beschreibung des phononischen Wärmetransportes über z.B. die BoltzmannTransport-Gleichung ermöglicht.[71] Eine solche Beschreibung ist aber nur solange gültig, wie wir die Gitterschwingungen auch als eigenständige Teilchen betrachten können. Denn in dieser Beschreibung stoßen die Phononen untereinander und an Defekten, aber können es werden keine wellenmechanischen Interferenzeffekte berücksichtigt. Diese treten jedoch spätestens auf, wenn die mittlere freie Weglänge der Phononen in der Größenordnung der Wellenlänge der Gitterschwingungen liegt. Dies ist zum Beispiel der Fall, wenn Grenzflächen zwischen Schichten mit unterschiedlichen elastischen Konstanten sehr nah (nur wenige Atomabstände) beieinander liegen. Wie das Verständnis des klassischen Wärmetransports für diese Spezialfälle angepasst werden kann, möchte ich später im Rahmen unseres Born-von-Kármán-Modells (Kapitel 2.2.3) zeigen. Zunächst folgen jedoch eine kurze Einführung in die nötigen Grundlagen. Dazu gehören beispielsweise die Phononendispersion des Festkörpers und eine generelle Definition des phononischen Wärmetransports.

\footnotetext{
${ }^{1}$ Siehe Zitat am Anfang dieser Arbeit.
} 


\subsubsection{Dispersion}

Die einfachste Näherung zur Beschreibung der Gitternormalschwingungen eines Festkörpers ist eine lineare Kette aus $N$ Atomen mit der Atommasse $m$ und dem Atomabstand $a$, die untereinander über Federn mit der Federkonstante $D$ verbunden sind. Die Auslenkung des $n$-ten Atoms aus seiner Ruhelage bezeichnen wir mit $u(n a)$. Die potentielle Energie, die in einer Feder gespeichert ist, beträgt $U_{\text {Feder }}=\frac{1}{2} D \cdot(\Delta u)^{2}$ mit der relativen Auslenkung aus der Ruhelage der Feder von $\Delta u=u(n+1)-2 u(n)+u(n-1)$. Die potentielle Energie unserer linearen Kette ist folglich

$$
U_{\text {Kette }, 1 \mathrm{D}}=\frac{D}{2} \sum_{n}[u(n a)-u((n+1) a)]^{2}
$$

und die Bewegungsgleichung des Atoms an der Position na ist

$$
m \ddot{u}(n a)=-\frac{\partial U_{\text {Kette, } 1 \mathrm{D}}}{\partial u(n a)}=-D[2 u(n a)-u((n+1) a)-u((n-1) a)] .
$$

Wie schon bei den Ultraschallschwingungen in Kapitel 2.1 wählen wir den Ansatz ebener Wellen $u(n a, t)=u_{0} e^{i(q n a-\omega t)}$ und setzen diesen in Gl. (2.16) ein:

$$
\begin{aligned}
m \omega^{2} & =D\left(2-e^{i q a}-e^{-i q a}\right) \\
\Rightarrow \quad & \omega=2 \sqrt{\frac{D}{m}}\left|\sin \left(\frac{q a}{2}\right)\right| .
\end{aligned}
$$

Am Anfang und Ende der Kette wählen wir die periodische Randbedingung $u(0)=$ $u(\mathrm{Na})$ wonach durch Einsetzen des Ansatzes die Bedingung

$$
e^{-i \omega t}=e^{i(q N a-\omega t)} \quad \Rightarrow \quad 1=e^{i q N a}
$$

folgt. Es gilt somit für $q$ :

$$
q N a=2 \pi j \quad \Rightarrow \quad q=\frac{2 \pi j}{N a}, \quad j \in \mathbb{Z}
$$

Unser Ansatz für $u$ ist jedoch mit einer Änderung von $q$ um $2 \pi / a$ periodisch. Es gibt folglich genau $N$ einzigartige Lösungen für $q$, die sich bei einer Verschiebung um $2 \pi / a$ wiederholen. Beim Vergleichen mit Gl. (2.17) sehen wir, dass es für alle 
(einzigartigen) Lösungen ausreicht $w(q)$ auf einem Intervall von $-\pi / a$ bis $\pi / a \mathrm{zu}$ betrachten. Eine solche Dispersion ist in Abbildung 2.8 dargestellt.

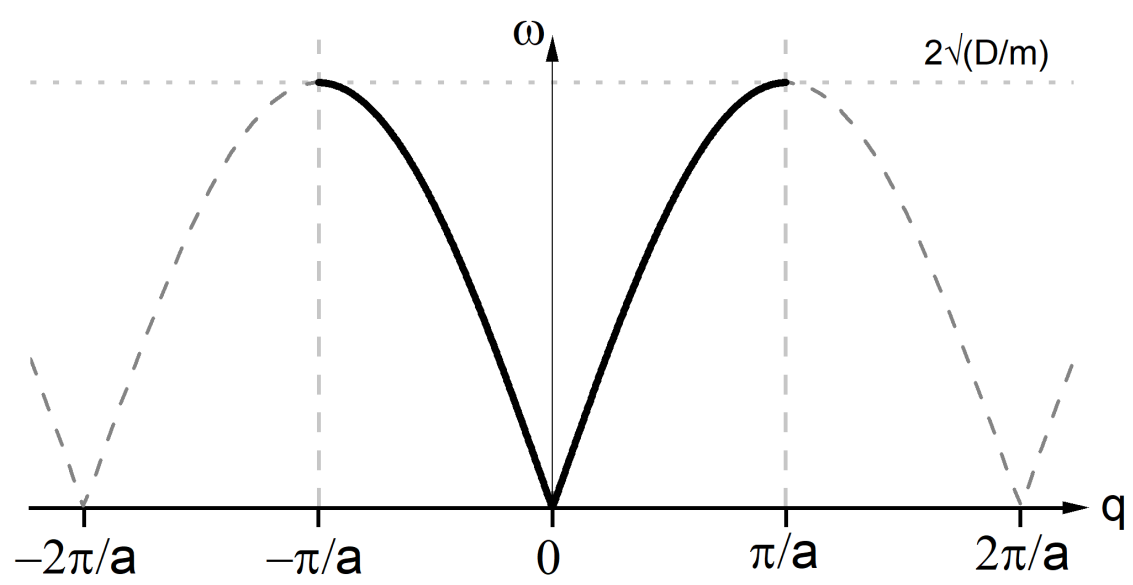

Abbildung 2.8: Dispersion $w(q)$ einer eindimensionalen Kette aus Atomen, beschrieben durch mit Federn verbundene Massen und periodischen Randbedingungen.

Im nächsten Schritt erweitern wir das Modell der linearen Kette indem wir sie aus zwei unterschiedlichen Atomen mit Massen $m_{1}$ und $m_{2}$ und der Federkonstante $D$ aufbauen. Dieses System lässt sich analog zum vorhergehenden Beispiel ausrechnen [12, 71]. Die Gitterkonstante $a$ ist nun nicht mehr der Abstand der einzelnen Atome, sondern der Abstand der kleinsten sich wiederholenden Struktur in unserer Kette, die sogenannte Basis. In unserem Beispiel umfasst die Basis das Atom der Masse $m_{1}$, der Masse $m_{2}\left(m_{1}<m_{2}\right)$, der Feder zwischen ihnen und die Feder vom zweiten Atom zum nächsten in der Kette. Die Auslenkung aus der Ruhelage des ersten Atoms bezeichnen wir als $u_{1}(n a)$ und des zweiten Atoms $u_{2}(n a)$. Das Gesamtpotential unserer Kette ist somit

$$
U_{\text {Kette Basis,1D }}=\frac{D}{2} \sum_{n}\left[u_{1}(n a)-u_{2}(n a)\right]^{2}+\frac{D}{2} \sum_{n}\left[u_{2}(n a)-u_{1}((n+1) a)\right]^{2}
$$

und die Bewegungsgleichungen der einzelnen Atome in der Basis:

$$
\begin{aligned}
& m_{1} \ddot{u}_{1}(n a)=-D\left[u_{1}(n a)-u_{2}(n a)\right]-D\left[u_{1}(n a)-u_{2}((n-1) a)\right] \\
& m_{2} \ddot{u}_{2}(n a)=-D\left[u_{2}(n a)-u_{1}(n a)\right]-D\left[u_{2}(n a)-u_{1}((n+1) a)\right]
\end{aligned}
$$


Als Ansatz wählen wir erneut ebene Wellen mit

$$
\begin{aligned}
& u_{1}(n a)=U_{1} e^{i(q n a-\omega t)} \\
& u_{2}(n a)=U_{2} e^{i(q n a-\omega t)}
\end{aligned}
$$

und den Amplituden $U_{1}$ und $U_{2}$.

Die Dispersion in diesem System ist [71]:

$$
\omega^{2}=D \mu \pm D \sqrt{\mu^{2}-\frac{4}{m_{1} m_{2}}} \sin ^{2}\left(\frac{q a}{2}\right)
$$

Dabei ist $\mu=\frac{1}{m_{1}}+\frac{1}{m_{2}}$. Die Dispersion ist in Grafik 2.9 dargestellt.

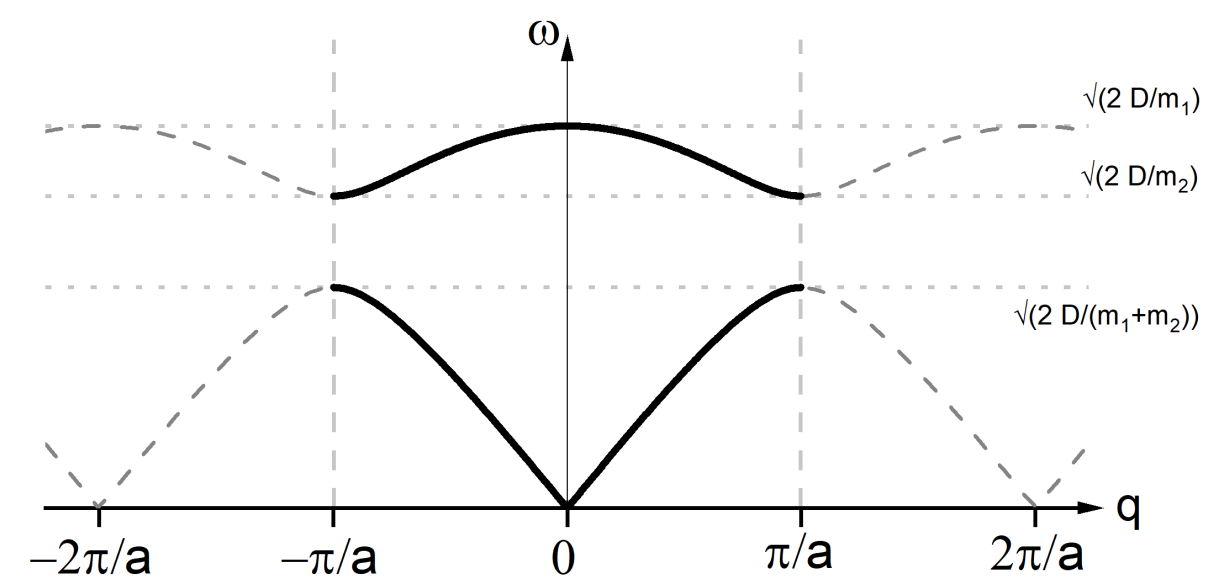

Abbildung 2.9: Dispersion $w(q)$ einer eindimensionalen Kette mit einer Basis aus zwei Atomen, beschrieben durch mit Federn verbundenen Massen und periodischen Randbedingungen.

Wie in Abb. 2.9 gut zu erkennen ist, teilt sich die Dispersion nun in zwei Bereiche auf: Einen niederfrequenten Zweig von 0 bis $\sqrt{2 D\left(m_{1}+m_{2}\right)^{-1}}$ und einen hochfrequenten Zweig zwischen $\sqrt{2 D m_{2}^{-1}}$ und $\sqrt{2 D m_{1}^{-1}}$. Die Gruppengeschwindigkeit $\partial \omega / \partial q$ des unteren Astes ist für kleine Frequenzen nahezu konstant, sowie wir es von akustischen Schallwellen her kennen. Diese Schwingungsmoden werden daher Akustische Moden genannt. Moden auf dem oberen Zweig bezeichnet man als Optische Moden. Dieser Name kommt von den Ionenkristallen mit zweiatomiger Basis in welchen die Schwingungen dieser Moden und das dadurch entstehende 
Dipolmoment der Atome typischerweise mit Infrarotlicht wechselwirkt [12]. Der optische Zweig ist im Vergleich zum akustischen Zweig flach und seine Moden haben daher eine nur geringe Gruppengeschwindigkeit. Bei $q=0$ und $q=\frac{\pi}{a}$ verschwindet die Gruppengeschwindigkeit sogar komplett. Einfach ausgedrückt: Im akustischen Zweig schwingen die einzelnen Einheitszellen (Basis) der Kette miteinander, im Optischen hingegen schwingen die Massen in der Basis gegeneinander. Wir sehen an diesem Beispiel, dass es für die Beschreibung von Effekte mit einem Hauptbeitrag der schnellen akustischen Wellen ausreichend ist, einzelne Atom-Massen innerhalb der Einheitszelle zu einer effektiven Gesamtmasse (in Abbildung 2.9 entsprechend $m_{1}+m_{2}$ ) zusammen zu fassen. Der Beitrag der langsamen optischen Moden wird in diesem Fall vernachlässigt.

Der Bereich zwischen den akustischen und den optischen Zweigen hat keine Schwingungszustände und wird wie in Kapitel 2.1 als Bandlücke bezeichnet.

Auch wenn dies eine sehr starke Vereinfachung zum Veranschaulichen der Phononenbandstruktur und ihren charakteristischen Punkten ist, so unterscheidet sich das Vorgehen bei einem realistischerem, dreidimensionalem Kugel-Federmodel nur wenig von der obigen Beschreibung. Durch komplexe Einheitszellen und ggf. richtungsabhängige Federpotentiale wird die Berechnung wesentlich unübersichtlicher und sei zunächst auf Kapitel 2.2.3 verschoben. Generell sei jedoch noch angemerkt, dass sich im dreidimensionalem Fall die Zweige in je drei Unterzweige aufteilen: Ein longitudinaler und zwei transversale Zweige. Für jedes zusätzliche Atom in der Einheitszelle erhalten wir zudem drei weitere solcher Zweige als optische Bänder. Bei $n$ Atomen in der Einheitszelle erhalten wir in unserer Dispersion somit drei akustische Zweige, von denen im isotropen Gitter die beiden transversalen Schwingungszweige entartet sind. Über diesen Bändern, getrennt von einer Bandlücke, erhalten wir weitere $3 n-3$ optische Bänder [12, 71].

\subsubsection{Wärmeleitfähigkeit der Phononen}

In einem Isolator wird die Wärmeleitfähigkeit durch den Transport über Gitterschwingungen dominiert. Im Teilchenbild liegt es zunächst nahe, die bosonischen Phononen in erster Näherung als ideales Gas zu betrachten. Die Wärmeleitfähigkeit $\kappa$ können wir hier als Produkt der Wärmekapazität des Phononengases $C$, der 
mittleren Geschwindigkeit der Phononen $v$ und ihrer mittleren freien Weglänge $l$ als

$$
\kappa=\frac{1}{3} C v l
$$

beschreiben [12]. Der Faktor 1/3 trägt den drei Raumfreiheitsgraden Rechnung. Wie wir in Kapitel 2.2.1 gesehen haben, sind die Gruppengeschwindigkeiten der Phononen von der Frequenz und dem Schwingungszweig abhängig. Auf dem optischen Zweig sind die Gruppengeschwindigkeiten klein im Vergleich zu dem akustischen Zweig. Der größte Beitrag zur Wärmeleitfähigkeit stammt also vom akustischen Zweig. In erster Näherung (für kleine $q$ ) entspricht also $v$ der Schallgeschwindigkeit des Volumenmaterials. Für $C$ wählen wir analog $C_{p}$ des Materials.

Wie bereits in der Einleitung erwähnt, ergibt das Bild der Phononen als eigenständige Teilchen nur Sinn, wenn die mittlere freie Weglänge $l$ deutlich kleiner ist als eine räumliche Einschränkung $d$ der Phononen. Dies kommt zum Beispiel vor, wenn $l$ kleiner als die Einschränkung durch Grenzflächen und Probenoberfläche ist. Konkret können wir uns folgende Fälle vorstellen:

- $d \gg l$ : Die Abstände von Grenzflächen sind größer als die mittlere freie Weglänge der Phononen. Wir erwarten „klassische“ diffusive Wärmeleitung und Zusammenhänge wie in Gl. ((2.24)).

- $d \approx l$ : Die Struktur ist in der Größenordnung der mittleren freien Phononenweglänge. Zusätzlich zu den Stößen und Streuprozessen die wir im Volumenmaterial beobachten, welche im letzten Fall $l$ ausgemacht haben, wird die mittlere freie Weglänge nun auch von Stößen an den Grenzflächen herabgesetzt. Experimente zeigen, dass $\kappa$ linear mit der Schichtdicke abnimmt [72]. Dieser Fall lässt sich noch im Teilchenbild der Phononen erklären.

- $l \gg d$ : Wenn die freie Weglänge größer als die Strukturgröße ist, kann ein Phonon z.B. nach der Reflexion an einer Grenzfläche mit sich selbst interferieren und wir können die Phononen nicht mehr im Sinne der kinetischen Gastheorie als eigenständige Teilchen beschreiben [32].

Diese Fälle sind vereinfacht und anschaulich in Abbildung 2.10 skizziert. 

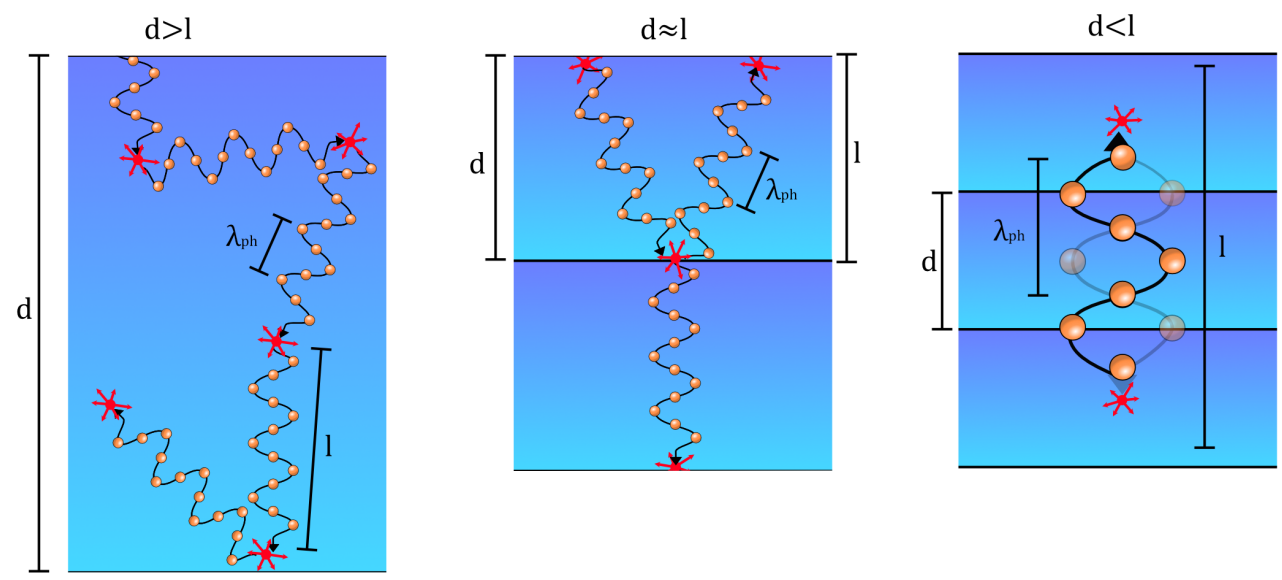

Abbildung 2.10: Links: Abstände von Grenzflächen und Oberflächen $d$ ist größer als die mittlere freie Weglänge $l$ der zum Wärmetransport beitragenden Phononen $l$. Außerdem ist die Wellenlänge $\lambda_{\text {ph }}$ deutlich kleiner als die mittlere freie Weglänge. Mitte: Abstände $d$ und freie Weglänge $l$ sind in derselben Größenordnung. Stöße an den Grenzflächen sorgen dafür, dass $l$ im Vergleich zum Volumenmaterial kleiner wird. Rechts: Die Grenzflächen sind kleiner als die Wellenlänge der am Wärmetransport beteiligten Phononen. Ein Teilchenbild ist aufgrund von Selbstinterferenz der Phononen an den Grenzflächen nicht mehr gerechtfertigt.

Betrachten wir an dieser Stelle die zwei Elemente Silizium und Germanium. Durch die Ähnlichkeit ihrer physikalischen Eigenschaften und ihrer Gitterstruktur (Diamantstruktur) eignen sich diese beiden Stoffe für einen direkten Vergleich. In Tabelle 2.1 sind die wichtigsten physikalischen Parameter kurz dargestellt und $l$ ist mit obiger Formel aus diesen Parametern berechnet.

Tabelle 2.1: Eine Übersicht der physikalischen Parameter von Silizium (Si) und Germanium (Ge) bei Raumtemperatur. Wenn nicht anders angegeben, stammen die Werte aus [73]. $l$ wurde mittels Gl. (2.24) berechnet.

\begin{tabular}{|c|c|c|c|c|c|}
\hline & $\mathrm{C}_{\mathrm{p}}\left[\mathrm{J} \mathrm{kg}^{-1} \mathrm{~K}^{-1}\right]$ & $v_{\mathrm{L}}\left[\mathrm{m} \mathrm{s}^{-1}\right]$ & $\kappa\left[\mathrm{W} \mathrm{m}^{-1} \mathrm{~K}^{-1}\right]$ & $\mathrm{a}[\mathrm{pm}]$ & $\mathrm{l}[\mathrm{nm}]$ \\
\hline \hline $\mathrm{Si}$ & 702 & $8436[74]$ & 148 & 543 & 32 \\
\hline $\mathrm{Ge}$ & 322 & $5400[75]$ & 60 & 566 & 20 \\
\hline
\end{tabular}


Im Sinne der Formel würde dies bedeuten, dass wir bei Schichten aus Silizium unter $32 \mathrm{~nm}$ und bei Germanium unter $20 \mathrm{~nm}$ eine Begrenzung der phononischen Wärmeleitfähigkeit durch die Schichtdicke erwarten müssen. Reale Wärmetransportmessungen zeigen jedoch, dass bereits ab einer Schichtdicke von $\sim 300 \mathrm{~nm}$ die Wärmeleitfähigkeit einer Siliziumschicht einbricht [72]. Dies legt nahe, dass die tatsächliche mittlere freie Weglänge der hauptsächlich am Wärmetransport beteiligten Phononen deutlich länger ist als in Tabelle 2.1 abgeschätzt.

In unserer Überschlagsrechnung haben wir $C(\omega)=C_{p}=$ konst. angenommen. Wie wir jedoch bereits festgestellt haben, trägt nicht jeder Phononenzweig gleich stark zum Wärmetransport bei. Mit der Annahme, dass die Energie gleichmäßig auf alle Phononenmoden aufgeteilt ist überschätzen wir also $C$ in Gl. (2.24), was wiederum zu einem Unterschätzen von $l$ führt. Die frequenzabhängige Wärmekapazität einzelner Phononenmoden lässt sich quantenmechanisch mit der Energie der Mode $\hbar \omega$ und der Bose-Einstein-Besetzung jeder einzelnen Mode

$$
n(q)=\frac{1}{e^{\frac{\hbar \omega(q)}{k_{\mathrm{B}} T}}-1}
$$

schreiben als:

$$
c_{v}(q)=\frac{\partial}{\partial T} \sum_{j} \int \frac{d^{3} q}{(2 \pi)^{3}} \hbar \omega_{j}(q) n_{j}(q)=\sum_{j} \int \frac{d^{3} q}{(2 \pi)^{3}} \hbar \omega_{j}(q) \frac{\partial n_{j}(q)}{\partial T} .
$$

Setzen wir Gl. (2.26) in Gl. (2.24) ein, und berücksichtigen, dass alle Moden mit einer nicht verschwindenden Geschwindigkeitskomponente $v_{z}^{j}(\omega)$ einen Beitrag zum Wärmetransport in $z$-Richtung leisten und $l$ aufgrund von Drei-Phonon-Streuung ebenfalls abhängig von $T$ und $\omega$ ist [76], können wir die Wärmeleitfähigkeit als [31]

$$
\kappa(T)=\sum_{j} \int \frac{d^{3} q}{(2 \pi)^{3}} \hbar \omega_{j}(q)\left|v_{z}^{j}(q)\right| l_{j}(T, q) \frac{\partial n\left(\omega_{j}(q), T\right)}{\partial T}
$$

schreiben. Für die allgemeine Form der phononischen Wärmeleitfähigkeit summieren wir über alle Zweige $j$ des Phononenspektrums. Für jeden Zweig integrieren wir über das Volumen der ersten Brillouinzone das Produkt aus Phononenenergien, Phononengruppengeschwindigkeit in z-Richtung $\left|v_{z}^{j}(q)\right|$, mittlere freie Weglänge $l_{j}(T, q)$ und die temperaturabhängige Änderung der Besetzung einzelner Moden. 
Bildlich ausgedrückt haben wir mit Gl. ((2.27)) einen semiklassischen Ausdruck dafür, wie viel thermische Leistung bei einem Temperaturungleichgewicht durch neu besetzte Phononenmoden in z-Richtung transportiert wird.

Bei Temperaturen $T>\frac{\hbar \omega}{k_{\mathrm{B}}}$ können wir $n(q) \approx \frac{k_{\mathrm{B}} T}{\hbar \omega(q)}$ nähern und $\kappa(T)$ vereinfacht sich $\mathrm{zu}$ :

$$
\begin{aligned}
\kappa(T) & =\sum_{j} \int \frac{d^{3} q}{(2 \pi)^{3}} \hbar \omega_{j}(q)\left|v_{z}^{j}(q)\right| l_{j}(T, q) \frac{k_{\mathrm{B}}}{\hbar \omega_{j}(q)} \\
\Rightarrow \kappa(T) & =k_{\mathrm{B}} \sum_{j} \int \frac{d^{3} q}{(2 \pi)^{3}}\left|v_{z}^{j}(q)\right| l_{j}(T, q)
\end{aligned}
$$

$\kappa$ ist bei $T>\frac{\hbar \omega}{k_{\mathrm{B}}}$ nicht direkt abhängig von den beteiligten Phononenfrequenzen, sondern von ihrer Gruppengeschwindigkeit und der temperaturabhängigen mittleren freien Weglänge. Mit typischen oberen Phononenfrequenzen im Bereich $\omega_{\max }=10 \mathrm{THz}-25 \mathrm{THz}[12]$ ist diese Formel ab Temperaturen von $T>200 \mathrm{~K}$ eine gute Näherung der Wärmeleitfähigkeit unter Berücksichtigung der Phononendispersion. Wir stellen des Weiteren fest, dass bei Raumtemperatur die akustischen Phononen mit hoher Gruppengeschwindigkeit den größten Beitrag zur Wärmeleitfähigkeit beitragen [76].

Die größte Unbekannte zur Berechnung der Wärmeleitfähigkeit nach Gleichung (2.28) ist neben dem Phononenspektrum die von Phononenmode und Temperatur abhängige mittlere freie Weglänge $l_{j}(T, q)$. Als Vereinfachung können wir für das exakte aber unbekannte Spektrum von $l_{j}(T, q)$ eine effektive mittlere freie Weglänge für alle zum thermischen Transport beitragenden Phononen $\xi(T)$ annehmen [15]. Wir definieren $\xi(T)$ als:

$$
\xi(T)=\frac{\sum_{j} \int \frac{d^{3} q}{(2 \pi)^{3}} \hbar \omega_{j}(q)\left|v_{z}^{j}(q)\right| l_{j}(T, q) \frac{k_{\mathrm{B}}}{\hbar \omega_{j}(q)}}{\sum_{j} \int \frac{d^{3} q}{(2 \pi)^{3}} \hbar \omega_{j}(q)\left|v_{z}^{j}(q)\right| \frac{k_{\mathrm{B}}}{\hbar \omega_{j}(q)}} .
$$


$\xi(T)$ soll uns im weiteren Verlauf als charakteristischer Vergleichswert für die typische Propagationslänge der am thermischen Transport beteiligten Phononen dienen. Die Gleichung (2.28) vereinfacht sich damit zu:

$$
\kappa(T)=k_{\mathrm{B}} \xi(T) \sum_{j} \int \frac{d^{3} q}{(2 \pi)^{3}}\left|v_{z}^{j}(q)\right| .
$$

Da alle Moden mit $\left|v_{z}^{j}(q)\right|>0$ einen Beitrag zur Wärmeleitfähigkeit in z-Richtung leisten, ist es offensichtlich, dass wir auch für eine eindimensionale Betrachtung des Wärmetransportes (z.B. bei einem dünnen Film) dennoch die komplette Dispersionsrelation benötigen. Im folgenden Kapitel werde ich ein hierfür geeignetes Modell zur Berechnung der 3D-Bandstruktur vorstellen.

\subsubsection{Born-von-Kármán-Modell}

Die letzte Zutat, die uns noch für eine Berechnung der Wärmeleitfähigkeit von realistischen Festkörpern fehlt, ist die dreidimensionale Dispersionsrelation der Phononen eines solchen Festkörpers. Zwar kann die Phononendispersion experimentell über inelastische Neutronenstreuung ermittelt werden [12], doch erfordert dies einen großen experimentellen Aufwand z.B. durch ein Experiment an einer Neutronenquelle [77, 78].

Eine deutlich weniger aufwendige und gleichzeitig flexiblere Möglichkeit ist es, die Dispersionskurven mit einem Modell des Festkörpers analytisch oder numerisch zu berechnen. Wie gut das Ergebnis einer solchen Berechnung ausfällt, hängt natürlich stark davon ab, wie realistisch das Modell die Wechselwirkung zwischen den Atomen und Elektronen im Kristall beschreiben kann. Eine Möglichkeit für ein solches Modell, welches ab-initio, also ohne Kenntnisse von materialspezifischen Konstanten auskommt, wäre die Dichtefunktionaltheorie (DFT) [79]. Diese Methode ist jedoch selbst sehr aufwendig und solche Berechnungen an phononischen Übergittern würden sicherlich eine eigenständige (theoretische) Dissertation rechtfertigen. 
Ein anderes Modell, welches allerdings nicht ohne experimentell ${ }^{2}$ bestimmte Parameter auskommt, ist das sogenannte Born-von-Kármán-Modell (BKM). Dieses Modell macht zwei Annahmen über den Festkörper:

- Born-Oppenheimer-Näherung: Aufgrund der Massendifferenz folgen die Elektronen den Bewegungen der Atome instantan wodurch ihre Bewegung für die mechanische Schwingung im Festkörper vernachlässigt werden kann.

- Von-Kármán-Näherung: Die Auslenkung der Atome von ihrer Ruhelage ist klein, sodass die Potentiale quadratisch angenähert werden können.

Tatsächlich haben wir diese beiden Näherungen bereits in Kapitel 2.2.1 in unserem Bild des Festkörpers als Kugel-Feder-Modell vorausgesetzt. Um unsere bekannte Bewegungsgleichung Gl. (2.16) auf den dreidimensionalen Fall zu erweitern, fassen wir zunächst alle Atomauslenkungen $u_{i}(\vec{r}, t)$ mit $i=x, y, z$ für das Atom an der Position $\vec{r}=(x, y, z)$ in den Vektor $\mathbf{u}(\vec{r}, t)$ zusammen. Die Bewegungsgleichung hat dann die Form:

$$
m_{k} \frac{\partial^{2} \mathbf{u}(\vec{r}, t)}{\partial t^{2}}=-\sum_{\alpha} D_{\alpha} \sum_{j=1 \ldots j_{\alpha}} \hat{\mathbf{n}}_{j}\left[\mathbf{u}(\vec{r})-\mathbf{u}\left(\vec{r}+\vec{n}_{j}\right)\right] \cdot \hat{\mathbf{n}}_{j} .
$$

$\vec{n}_{j}$ ist der Vektor zum $j$ ten Nachbaratom und $\hat{n}_{j}$ der normierte Richtungsvektor. $\alpha$ ist ein Index, welcher für die Art der Wechselwirkung steht. Die Summe geht über die nächsten, die übernächsten und die über-übernächsten Nachbarn. Für jede dieser Wechselwirkungen definieren wir eine eigene Kopplungskonstante $D_{\alpha}$ (analog zur Federkonstante in Kapitel 2.2.1). Je nach Art des Gitters (kubisch, rhomboedrisch, usw.) läuft der Index $j_{\alpha}$ über die Anzahl der durch $\alpha$ bestimmten Nachbarn. In drei Dimensionen und bei einem kubischen Gitter sind dies respektive 6, 12 und 8 Nachbarn.

\footnotetext{
${ }^{2}$ oder per ab-initio Berechnungen bestimmte Parameter.
} 


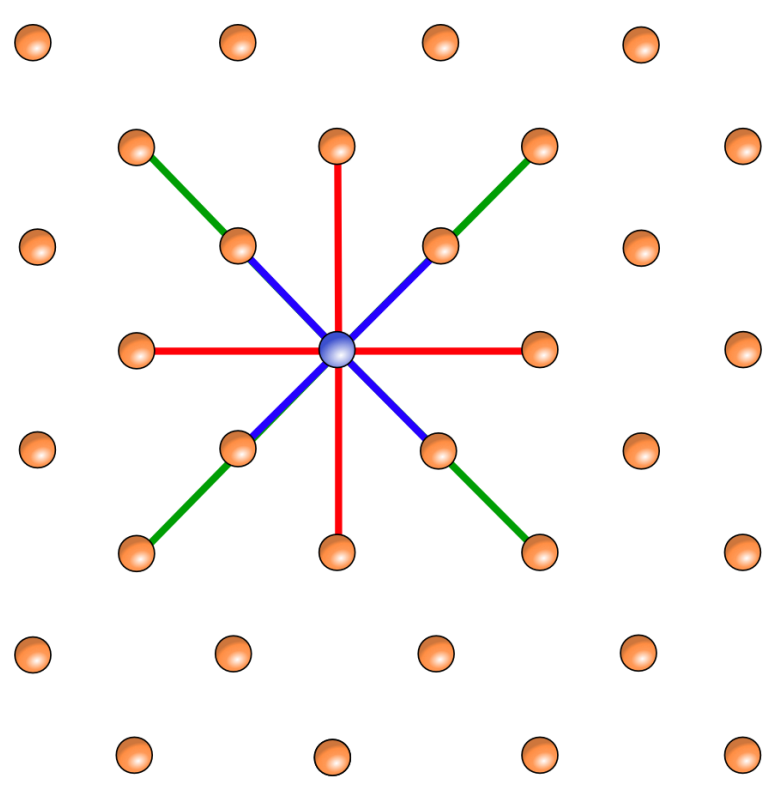

Abbildung 2.11: Verbindung zum nächsten Nachbaratom (blau), übernächsten Nachbaratom (rot) und über-übernächsten Nachbaratom (grün) dargestellt in 2D.

Mit dem Ansatz ebener Wellen $u(\vec{r})=u_{0} \cdot e^{i \cdot \vec{q} \cdot \vec{r}}$ erhalten wir aus der Gleichung (2.31) ein Gleichungssystem in Form der $3 k \times 3 k$ Matrix $\mathbf{M}(q)$. Die Phononendispersion erhalten wir als Lösung des Eigenwertproblems

$$
\mathbf{M}(q) \cdot \vec{e}=\omega^{2} \vec{e}
$$

Mit geeigneten numerischen Bibliotheken lässt sich der Eigenvektor als Funktion von $q$ mit akzeptablem Rechenaufwand berechnen (Details zu der in dieser Arbeit verwendeten Implementation des Modells ist im Anhang A.5 und in [80] zu finden). Dies ermöglicht das Rastern durch die erste Brillouinzone und so das Berechnen einer dreidimensionalen Phononendispersion für ein beliebiges Atomgitter.

Wie ich in Kapitel 2.2.2 anhand von Gleichung (2.28) bereits angemerkt habe, haben Phononen mit hoher Gruppengeschwindigkeit den größten Einfluss auf die Wärmeleitfähigkeit. Daher können wir optische Moden mit kleiner Gruppenge- 
schwindigkeit in unseren Berechnungen zur Wärmeleitfähigkeit des Gitters vernachlässigen. Durch Zusammenfassen einzelner Atome zu einer effektiven Masse der Einheitszelle, reduziert sich die Anzahl der Eigenwerte um $3 n-3$ mit $n$ der Anzahl der Atome in der Basis. Beispielsweise können wir eine kubische Einheitszelle mit der Perovskitstruktur $\mathrm{ABO}_{3}$ und den zugehörigen Atommassen $m_{\mathrm{A}}, m_{\mathrm{B}}$ und $m_{\mathrm{O}}$ zu einer effektiven Masse $m_{\mathrm{ABO}_{3}}=m_{\mathrm{A}}+m_{\mathrm{b}}+3 \cdot m_{\mathrm{O}}$ zusammenfassen. Erinnern wir uns an Abb. 2.9, beeinflusst diese Vereinfachung die akustischen Zweige nicht.

Zwei solcher so berechneten Bandstrukturen sind exemplarisch in Abbildung 2.12 für kubisches LMO und in Abbildung 2.13 für kubisches SMO dargestellt. Die Kopplungskonstanten (siehe Tabelle A.3 im Anhang) wurden mit Hilfe von elastischen Parametern aus DFT-Berechnungen [81, 82] bestimmt. 


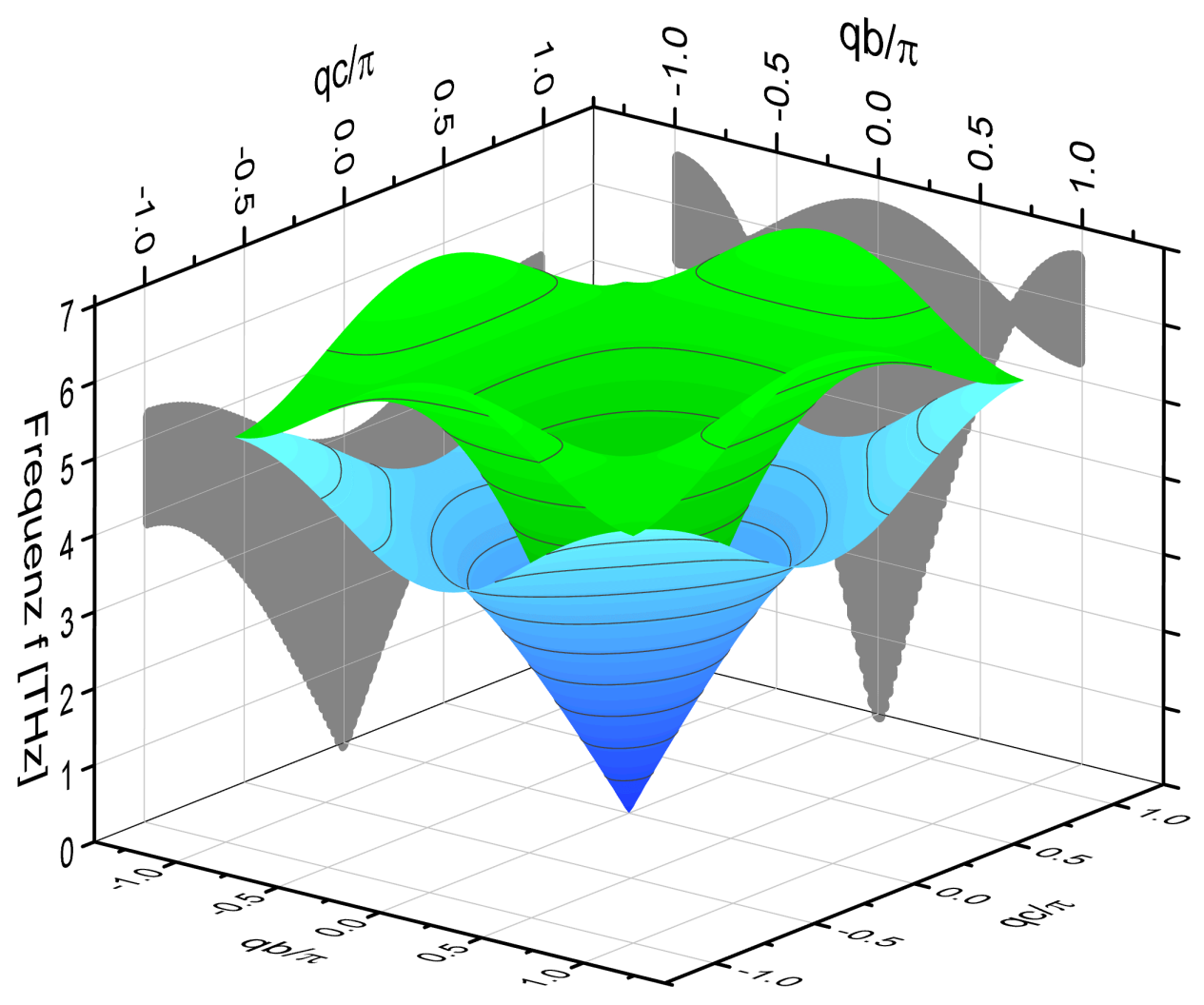

Abbildung 2.12: Phononenbandstruktur von $\mathrm{LaMnO}_{3}$ in der kubischen $(\mathrm{Pm} \overline{3} \mathrm{~m})$ Struktur in den Richtungen $q_{\mathrm{z}}$ und $q_{\mathrm{y}}$. a, b und c sind die Gitterkonstanten in die jeweilige Richtung $\mathrm{x}, \mathrm{y}$ und $\mathrm{z}$. Die $q_{\mathrm{x}}$ Achse ist identisch mit den anderen Richtungen und wurde aus Darstellungsgründen weggelassen. Grün ist der longitudinale Schwingungszweig, blau der zweifach entartete transversale Zweig. Der longitudinale Zweig ist auf die $q_{\mathrm{z}}$-Ebene und die transversalen Zweige auf die $q_{\mathrm{y}}$-Ebene projiziert. 


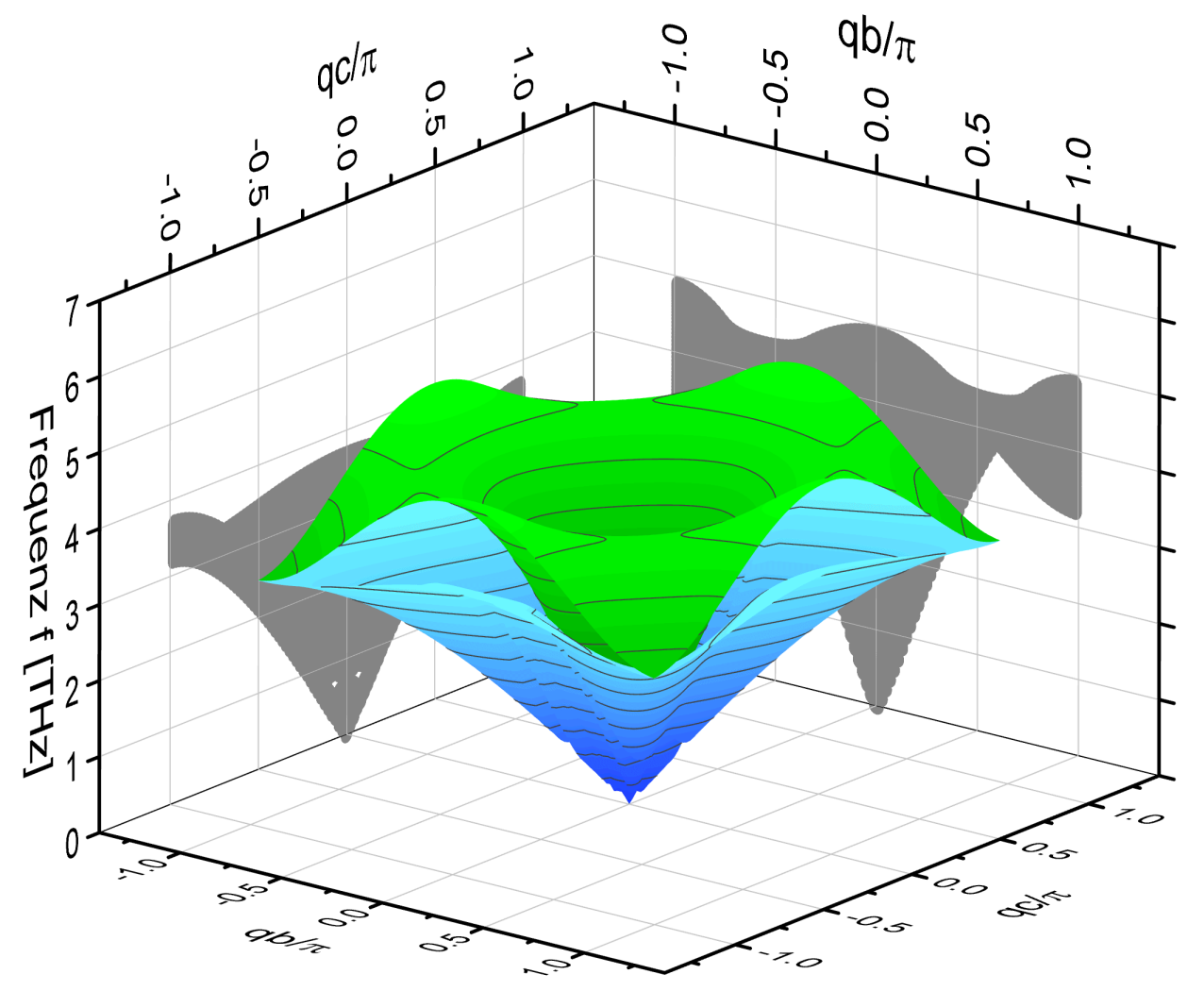

Abbildung 2.13: Phononenbandstruktur von $\mathrm{SrMnO}_{3}$ in der kubischen $(\mathrm{Pm} \overline{3} \mathrm{~m})$ Struktur in den Richtungen $q_{\mathrm{z}}$ und $q_{\mathrm{y}}$. a, b und c sind die Gitterkonstanten in die jeweilige Richtung $\mathrm{x}, \mathrm{y}$ und $\mathrm{z}$. Die $q_{\mathrm{x}}$ Achse ist identisch mit den anderen Richtungen und wurde aus Darstellungsgründen weggelassen. Grün ist der longitudinale Schwingungszweig, blau der zweifach entartete transversale Zweig. Der longitudinale Zweig ist auf die $q_{\mathrm{z}}$-Ebene und die transversalen Zweige auf die $q_{\mathrm{y}}$-Ebene projiziert. 
Gut zu erkennen ist der schnelle longitudinale Dispersionszweig (grün) und die langsameren und entarteten transversalen Schwingungszweige (blau). Für einen genaueren Vergleich von LMO und SMO, schauen wir uns in Abb. 2.14 direkt die Phononendispersion am Schnittpunkt mit der Ebene $\left(q_{\mathrm{x}}, q_{\mathrm{y}}, 0\right)$, also der Dispersion entlang $q_{\mathrm{z}}$ Richtung an. In dieser Grafik dargestellt sind die longitudinalen (LA)

(a)

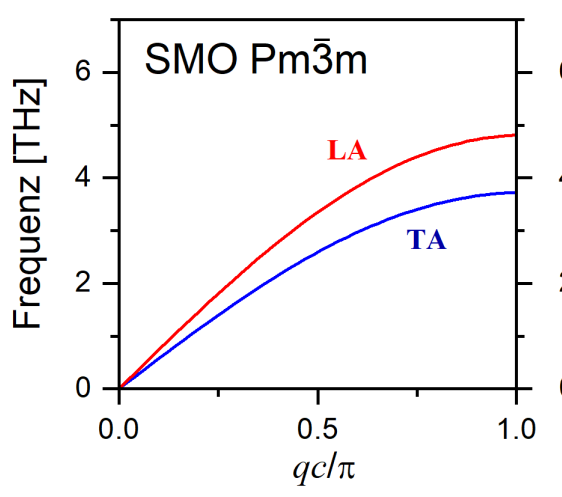

(b)

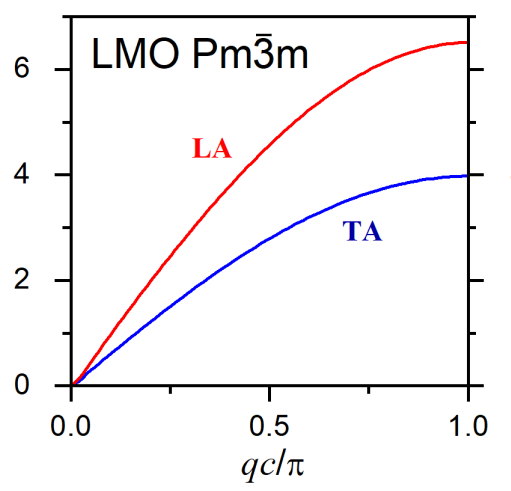

(c)

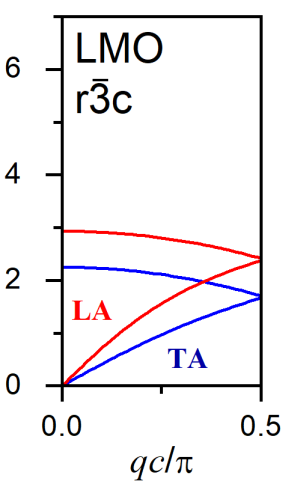

Abbildung 2.14: (a) Dispersion $\omega(q) / 2 \pi$ für kubisches (Pm $\overline{3} \mathrm{~m}) \mathrm{SrMnO}_{3}$, (b) kubisches $(\mathrm{Pm} \overline{3} \mathrm{~m}) \mathrm{LaMnO}_{3}$ und (c) rhomboedrisches $(\mathrm{r} \overline{3} \mathrm{c}) \mathrm{LaMnO}_{3}$.

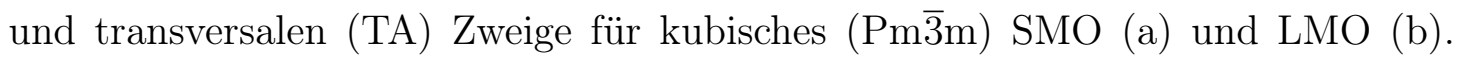
Bei Raumtemperatur kommt LMO als Einzelfilm oder in Übergittern häufig in einer rhomboedrischen $\mathrm{r} \overline{\mathrm{C}} \mathrm{c}$ Phase vor [83-85]. LMO mit dieser Struktur ist in (c) dargestellt.

Zusammenfassend sei gesagt, dass unser Modell es erlaubt, durch Anpassen der Nächstenachbarvektoren $\vec{n}$ (und deren Anzahl $j_{\alpha}$ ) beliebige Kristallstrukturen, und durch Variation von $m_{k}$ sowie den Kopplungskonstanten $D_{\alpha}$ auch beliebige Kombinationen von unterschiedlichen Atomen zu berechnen. Dies kann beispielweise ein Übergitter aus unterschiedlichen Atomen sein. In Abb. 2.15 ist beispielsweise eine Auswahl von berechneten Bändern eine $(\mathrm{LMO})_{2} /(\mathrm{SMO})_{2}$-Übergitter dargestellt. Gut zu erkennen ist Reduktion der Gruppengeschwindigkeit aufgrund der Übergitterstruktur im longitudinalen Ast (orange) in $q_{\mathrm{z}}$-Richtung. 


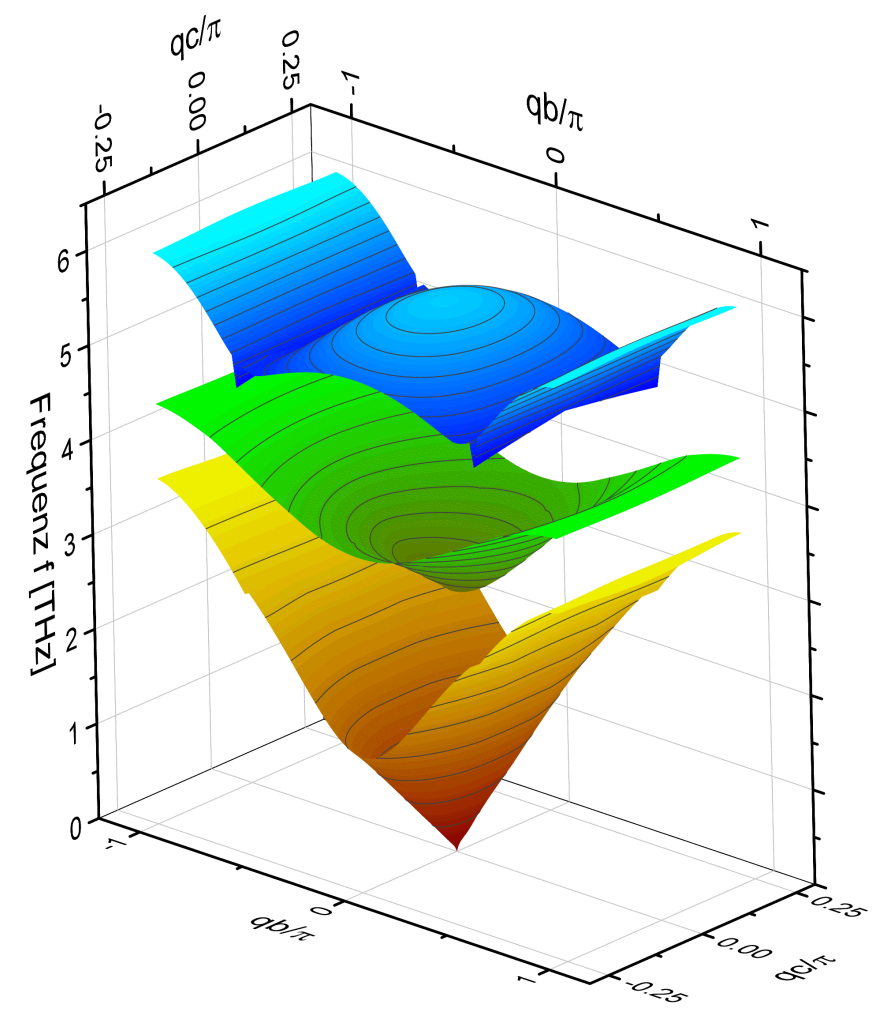

Abbildung 2.15: Auswahl aus der Phononenbandstruktur eines $(\mathrm{LMO})_{2} /(\mathrm{SMO})_{2^{-}}$ Übergitter in den Richtungen $q_{\mathrm{z}}$ und $q_{\mathrm{y}}$. a, b und $\mathrm{c}$ sind die Gitterkonstanten in die jeweilige Richtung $\mathrm{x}, \mathrm{y}$ und $\mathrm{z}$. Die $q_{\mathrm{x}}$ Achse ist identisch mit der $q_{\mathrm{y}}$-Achse und wurde aus Darstellungsgründen weggelassen.

Die von uns verwendeten Kopplungsparameter sind in Tabelle A.3 im Anhang aufgelistet.

Ein Nachteil des Modells ist, dass Wechselwirkungen mit langer Reichweite, wie z.B. Coulombwechselwirkung, sich nur schlecht in einem Feder-Masse-Modell mit wenigen Nachbarn berücksichtigen lassen. Da wir uns bei der Betrachtung des thermischen Transports auf die akustischen Äste beschränken können (siehe Kapitel 2.2.1 und 2.2.2) ist es ausreichend in unserem Feder-Masse-Modell der Übergitter ausschließlich die ladungsneutralen Einheitszellen als schwingende Massen zu berücksichtigen und den Beitrag von Wechselwirkungen mit langer Reichweite auf das (akustische) Phononenspektrum zu vernachlässigen. 


\section{Kapitel 3}

\section{Experimentelle Methoden}

Nachdem ich im letzten Kapitel ein wenig die Theorie beleuchtet und generelle Begriffe wie akustische Phononen oder Phononenbandlücke eingeführt habe, möchte ich in diesem Kapitel nun auf die experimentellen Methoden eingehen.

Ich werde zunächst die von mir in dieser Arbeit untersuchten Probensysteme vorstellen. Dies sind konkret die Multilagen aus $\mathrm{ZrO}_{2} / \mathrm{MgO}$ und die Übergitter aus $\mathrm{LaMnO}_{3}$ und $\mathrm{SrMnO}_{3}$.

Auch wenn die Probenherstellung zum Großteil von Projektpartnern im SFB 1073 durchgeführt wurde, möchte ich natürlich für ein umfangreiches Bild die genutzten Herstellungsmethoden kurz beschreiben. Dazu gehört unter anderem Pulsed-LaserDeposition (PLD) für die Multilagen und die Metallorganische Aerosoldeposition (MAD) für die Übergitter. Die Herstellung der Kupferschichten für das TTRExperiment mittels Elektronenstrahlverdampfen werde ich detaillierter beschreiben, da ich diese UHV-Anlage im Rahmen meiner Dissertation selbst konstruiert und zum Herstellen der Schichten verwendet habe.

Nach den Kapiteln zur Probenherstellung folgt die Beschreibung der eigentlichen Messmethoden. Dazu gehört die Ultrakurzzeitspektroskopie (Pump-ProbeReflektivität, kurz PPR) zum Messen der elastischen Probendynamik und die Transiente Thermoreflektivität (TTR) zur Bestimmung der Wärmeleitfähigkeit von Übergittern. Die numerische Auswertungsmethode der Thermoreflektivität werde ich ebenfalls kurz vorstellen.

Anschließend folgt eine kurze Übersicht über numerischen Methoden zur Simulation von Probendynamik über eine Finite-Differenzen Simulation und die Be- 
rechnung von phononischen Bandstrukturen durch ein Born-von-Kármán-Modell (BKM). Auch wenn man argumentieren kann, dass diese Computersimulation eigentlich Teil der Theorie ist, so sehe ich sie als einen unterstützenden Teil der experimentellen Methoden und als Ergänzung der Messaufbauten.

\subsection{Probenherstellung}

\subsubsection{Probenübersicht}

Für die Untersuchungen der akustischen Ultraschalldynamik in Multilagensystemen wurden von der Gruppe um Prof. Dr. Krebs des Instituts für Materialphysik Multilagen aus Zirkondioxid $\left(\mathrm{ZrO}_{2}\right)$ und Magnesiumoxid $(\mathrm{MgO})$ mit verschiedenen Doppellagendicken mittels Pulsed-Laser-Deposition (PLD) hergestellt [86]. Über eine verfahrbare Blende wurde zudem eine Wolfram-Schicht mit variabler Dicke im Bereich von $4.5 \mathrm{~nm}-18 \mathrm{~nm}$ auf diese Multilagen aufgebracht [26]. Mit der PLDMethode wurden außerdem Referenzproben verschiedener Dicken aus $\mathrm{ZrO}_{2}$ hergestellt, welche mir in Kapitel 3.4.1.4 zur Kontrolle des TTR-Messaufbaus und zur Überprüfung der TTR-Auswertungsmethode dienen.

Die Übergitterproben mit unterschiedlichen Doppellagen aus $\mathrm{LaMnO}_{3}$ und $\mathrm{SrMnO}_{3}$ wurden von der Gruppe von Prof. Dr. Moshnyaga mit Hilfe der Metallorganischen Aerosoldeposition (MAD) für unser Projekt im Sonderforschungsbereich 1073 (A02) hergestellt und mit Hilfe von Röntgendiffraktometrie (XRD), Röntgenreflektometrie (XRR) und Rasterkraftmikroskopie charakterisiert.

Als Substrat für diese Proben dient je ein $10 \mathrm{~mm}$ x $5 \mathrm{~mm}$ x $0.5 \mathrm{~mm}$ großer Strontiumtitanat $\left(\mathrm{SrTiO}_{3}\right)$ Kristall mit ( $\left.\begin{array}{lll}1 & 0 & 0\end{array}\right)$ Orientierung. Diese Übergitter wurden in vielfältiger Variation hergestellt. Die von mir untersuchten und in dieser Arbeit vorgestellten Proben sind in Tabelle 3.1 aufgeführt.

Für die Messungen zur Wärmeleitfähigkeit im TTR wurden die Übergitter mit je $50 \mathrm{~nm}$ Kupfer in einer UHV-Anlage zum Elektronenstrahlverdampfen (EBPVD) beschichtet. 


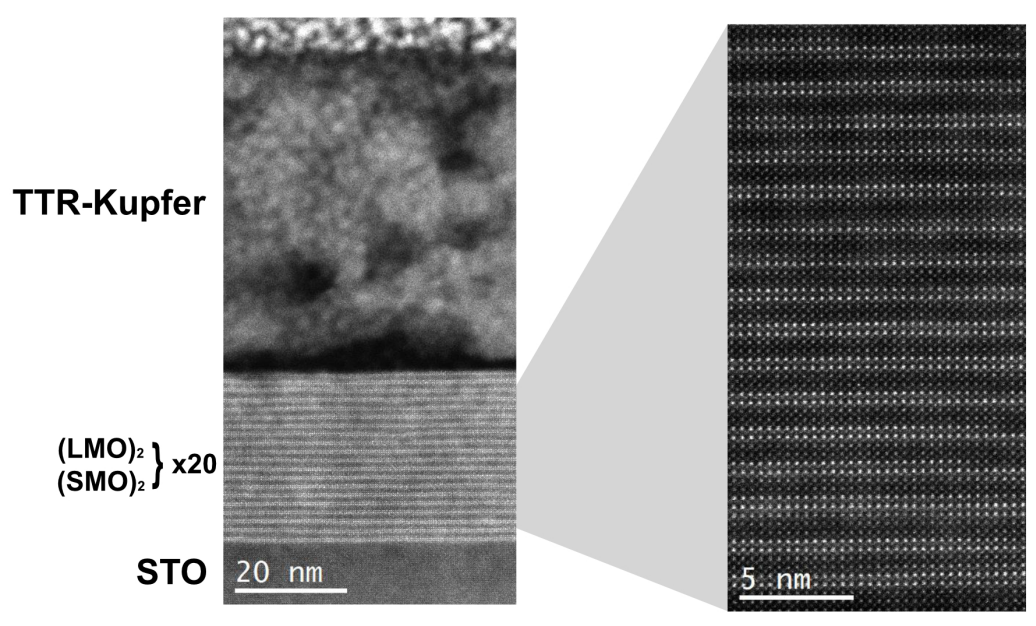

Abbildung 3.1: Transmissionselektronenmikroskop-Aufnahmen einer $\left[(\mathrm{LMO})_{2} /(\mathrm{SMO})_{2}\right]_{20}$ Übergitter-Probe. Im linken Teil des Bildes ist ein Querschnitt der kompletten Probe mit STO-Substrat, LMO/SMO-Übergitter und Kupferschicht für die TTR-Messung zu sehen. Der rechte Teil des Bildes ist ein vergrößerter Ausschnitt des Übergitters. Die Aufnahmen wurden von Robert Gruhl angefertigt und zur Verfügung gestellt.

Die Benennung der Proben im weiteren Verlauf meiner Arbeit erfolgt nach dem Schema:

$$
\left[(\mathrm{A})_{\mathrm{n}} /(\mathrm{B})_{\mathrm{m}}\right]_{k}
$$

Dabei steht die eckige Klammer für den Aufbau der Doppellage und $k$ für die Wiederholungen der Doppellage. A und B bezeichnet die Materialien aus denen die Doppellage aufgebaut ist und $n$ sowie $m$ die einzelne Schichtdicke des Materials. Sofern $n$ und $m$ ohne Einheit steht, ist die Anzahl an atomaren Monolagen bzw. Einheitszellen des Materials in Richtung senkrecht zum Substrat gemeint. Wenn es für das Verständnis nicht von Bedeutung ist, werde ich im Fließtext die eckige Klammern um die Übergitter weglassen (beispielweise $(\mathrm{LMO})_{6} /(\mathrm{SMO})_{3}$ anstelle von $\left.\left[\mathrm{LMO}_{6} / \mathrm{SMO}_{3}\right]_{10}\right)$. Die Anzahl der Lagen kann jederzeit in der Tabelle 3.1 nachgeschlagen werden. 
Tabelle 3.1: Eine Übersicht der unterschiedlichen Proben. Für $m / n=1$ Übergitter wurden zwei Probenreihen untersucht. Eine $\left[\mathrm{LMO}_{2} / \mathrm{SMO}_{2}\right]_{20}$ Probe zeigt eine antiferromagnetische Phase (AFM) und wurde in der Tabelle gekennzeichnet. $c$ ist die mittlere Gitterkonstante der Übergitter.

\begin{tabular}{|c|c|c|c|c|c|c|}
\hline Name & Art & $\mathrm{m}$ & $\mathrm{n}$ & $\mathrm{k}$ & $\mathrm{d}[\mathrm{nm}]$ & $\mathrm{c}[\mathrm{nm}]$ \\
\hline$\left[(\mathrm{MgO})_{10 \mathrm{~nm}} /\left(\mathrm{ZrO}_{2}\right)_{10 \mathrm{~nm}}\right]_{50}$ & Multilage & $10 \mathrm{~nm}$ & $10 \mathrm{~nm}$ & 50 & & \\
\hline$\left[(\mathrm{MgO})_{5 \mathrm{~nm}} /\left(\mathrm{ZrO}_{2}\right)_{5 \mathrm{~nm}}\right]_{50}$ & Multilage & $5 \mathrm{~nm}$ & $5 \mathrm{~nm}$ & 50 & & \\
\hline$\left(\mathrm{ZrO}_{2}\right)_{50 \mathrm{~nm}}$ & Schicht & $50 \mathrm{~nm}$ & - & - & & \\
\hline$\left(\mathrm{ZrO}_{2}\right)_{200 \mathrm{~nm}}$ & Schicht & $200 \mathrm{~nm}$ & - & - & & \\
\hline$\left(\mathrm{ZrO}_{2}\right)_{500 \mathrm{~nm}}$ & Schicht & $500 \mathrm{~nm}$ & - & - & & \\
\hline$\left(\mathrm{ZrO}_{2}\right)_{1000 \mathrm{~nm}}$ & Schicht & $1000 \mathrm{~nm}$ & - & - & & \\
\hline$\left[\mathrm{LMO}_{3} / \mathrm{SMO}_{3}\right]_{10}$ & Übergitter & 3 & 3 & 10 & 23.2 & 0.382 \\
\hline$\left[\mathrm{LMO}_{4} / \mathrm{SMO}_{4}\right]_{10}$ & Übergitter & 4 & 4 & 10 & 30.9 & 0.382 \\
\hline$\left[\mathrm{LMO}_{5} / \mathrm{SMO}_{5}\right]_{10}$ & Übergitter & 5 & 5 & 10 & 38.5 & 0.382 \\
\hline$\left[\mathrm{LMO}_{10} / \mathrm{SMO}_{10}\right]_{10}$ & Übergitter & 10 & 10 & 10 & 77.0 & 0.382 \\
\hline$\left[\mathrm{LMO}_{1} / \mathrm{SMO}_{1}\right]_{40}$ & Übergitter & 1 & 1 & 40 & 31.0 & 0.382 \\
\hline$\left[\mathrm{LMO}_{2} / \mathrm{SMO}_{2}\right]_{20}$ & Übergitter & 2 & 2 & 20 & 29.0 & 0.382 \\
\hline$\left[\mathrm{LMO}_{2} / \mathrm{SMO}_{2}\right]_{20}+\mathrm{AFM}$ & Übergitter & 2 & 2 & 20 & 31.6 & 0.382 \\
\hline$\left[\mathrm{LMO}_{3} / \mathrm{SMO}_{3}\right]_{10}$ & Übergitter & 3 & 3 & 10 & 22.3 & 0.382 \\
\hline$\left[\mathrm{LMO}_{4} / \mathrm{SMO}_{4}\right]_{10}$ & Übergitter & 4 & 4 & 10 & 28.3 & 0.382 \\
\hline$\left[\mathrm{LMO}_{5} / \mathrm{SMO}_{5}\right]_{10}$ & Übergitter & 5 & 5 & 10 & 37.4 & 0.382 \\
\hline$\left[\mathrm{LMO}_{6} / \mathrm{SMO}_{6}\right]_{10}$ & Übergitter & 6 & 6 & 10 & 42.0 & 0.382 \\
\hline$\left[\mathrm{LMO}_{8} / \mathrm{SMO}_{4}\right]_{10}$ & Übergitter & 8 & 4 & 10 & 46.0 & 0.384 \\
\hline$\left[\mathrm{LMO}_{10} / \mathrm{SMO}_{5}\right]_{10}$ & Übergitter & 10 & 5 & 10 & 57.9 & 0.384 \\
\hline$\left[\mathrm{LMO}_{12} / \mathrm{SMO}_{6}\right]_{10}$ & Übergitter & 12 & 6 & 10 & 69.5 & 0.385 \\
\hline$\left[\mathrm{LMO}_{20} / \mathrm{SMO}_{10}\right]_{10}$ & Übergitter & 20 & 10 & 10 & 116 & 0.385 \\
\hline
\end{tabular}




\subsubsection{Pulsed Laser Deposition}

Die in meiner Dissertation untersuchten $\mathrm{W} /\left[\mathrm{MgO} / \mathrm{ZrO}_{2}\right]$ Multilagen wurden von der Gruppe um Prof. Dr. Krebs mittels der PLD-Methode hergestellt [87]. Bei dieser Depositionsmethode wird ein leistungsstarker, gepulster Laserstrahl in eine Ultrahochvakuum-Anlage auf das zu deponierende Material fokussiert. Die absorbierte Energie führt im Targetmaterial zur Bildung eines Plasmas und so dazu, dass ein Teil das Materials in die Gasphase übergeht. Vom Target bewegt sich das ausgestoßene Material zum Substrat, an dem es kondensiert und eine dünne Schicht bildet. Dieses Verfahren gehört, wie auch das Elektronenstrahlverdampfen, zu der Gruppe der physikalischen Gasphasenabscheidung.

Der für die Proben verwendete KrF Excimer Laser hat eine Wellenlänge von $248 \mathrm{~nm}$, eine Repetitionsrate von $10 \mathrm{~Hz}$ und eine Pulslänge von $20 \mathrm{~ns}$. Durch das computergesteuerte Umlenken des Laserstrahls lassen sich automatisiert und ohne Brechen des Vakuums Multilagen mit mehreren Wiederholungen herstellen. Der Wachstumsprozess wird von einer Schwingquarzwaage überwacht.[86]

Die Wolframdeckschichten mit variabler Dicke wurden durch das Verfahren einer Shutterblende vor der Probe hergestellt [26]. Die Schichtdicke steigt auf der 10/10 Probe von $9 \mathrm{~nm}$ auf $18 \mathrm{~nm}$ und auf der $5 / 5$ Probe von $4.5 \mathrm{~nm}$ auf $9 \mathrm{~nm}$.

\subsubsection{Metallorganische Aerosoldeposition (MAD)}

Zur Herstellung von Schichten mit der Metallorganischen Aerosoldeposition (MAD) wird ein metallhaltiger Präkursor in einem Lösungsmittel (Dimethylformamid) gelöst. Für die LMO/SMO Übergitter kamen dabei Lanthan-, Strontium- und Manganacetylacetonatpräkusoren zum Einsatz. Die Präkursoren werden je nach herzustellender Schicht gemischt ${ }^{1}$ und in ein Mehrspitzensystem gefüllt. Von dort aus werden sie computergesteuert über eine Düse auf das geheizte Substrat gespritzt. Dort findet eine Pyrolysereaktion statt, welche zu einem epitaktischen Wachstum von Oxidschichten aus den Metallen in der Präkursorlösung und dem Sauerstoff der Umgebung führt. Über eine Ellipsometriemessung kann während der Deposition das Schichtwachstum kontrolliert werden. Die computergesteuerte Deposition über

\footnotetext{
${ }^{1}$ Das genaue Mischverhältnis wird experimentell anhand von Toolingproben bestimmt [88].
} 
die Spritzen erlaubt so das Wachsen von oxidischen Filmen mit monolagengenauer Präzision [88].

Für die Details zur MAD-Methode verweise ich an dieser Stelle auf die Arbeit von Markus Jungbauer et al. [88] oder neuere Arbeiten von Marius Keunecke et al. [85].

\subsubsection{UHV-Elektronenstrahlverdampfen}

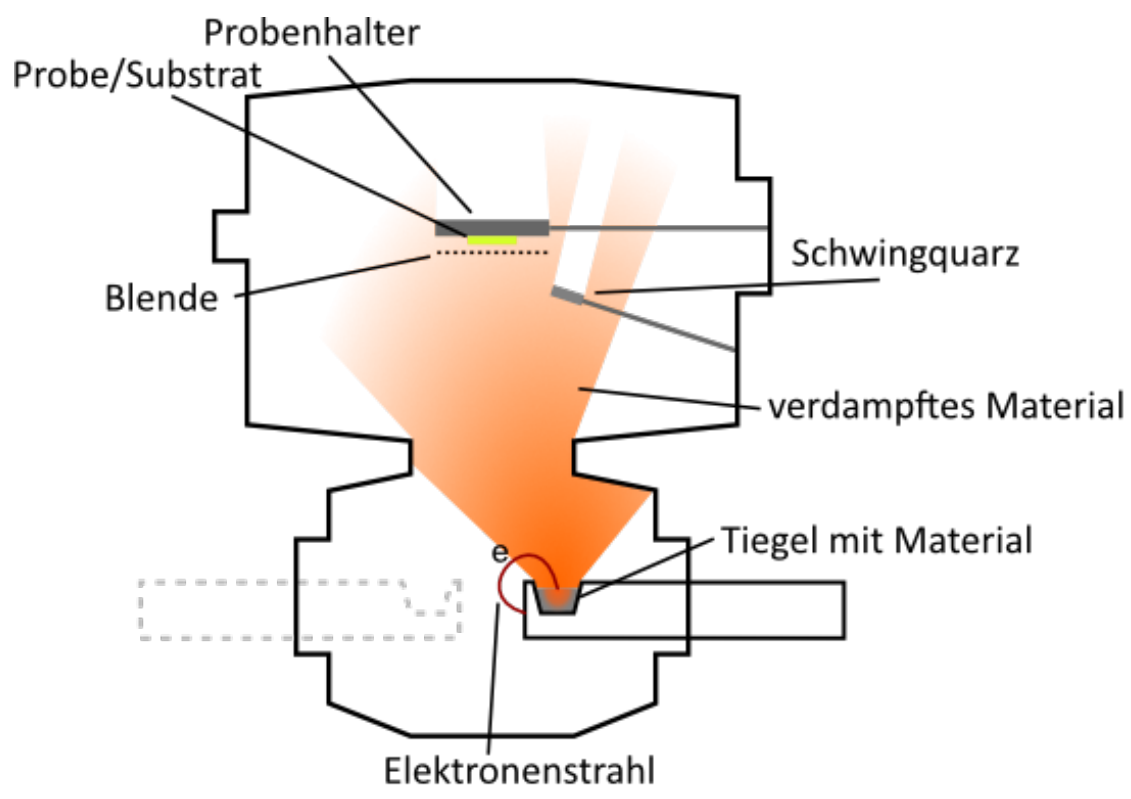

Abbildung 3.2: Vereinfachte Skizze der EBPVD-UHV-Kammer. Dargestellt ist die Probe (gelb) und die quasi ballistische Flugbahn des verdampften Materials (orange). Die Anlage verfügt über drei Elektronenstrahlverdampfer (in der Skizze angedeutet).

Für das Aufbringen der $50 \mathrm{~nm}$ Kupferfilme für das TTR-Experiment verwende ich eine UHV-Anlage, welche ich ursprünglich im Rahmen meiner Masterarbeit [64] für die Deposition von Wolfram aufgebaut habe. Während meiner Promotion habe ich die Anlage nicht nur weiter genutzt und gepflegt, sondern auch Verbesserungen an Anlage und Software vorgenommen. In diesem Kapitel werde ich mich darauf beschränken, den Zustand der Anlage zum Zeitpunkt des Schreibens darzustellen. Die meisten Filme wurden mit der Anlage in dieser Form hergestellt. 
Eine Übersicht aller relevanten Komponenten ist in Abbildung 3.2 dargestellt. Die Hauptkammer besteht aus zwei Töpfen, ein unterer Topf mit drei Elektronenstrahlquellen, und ein oberer Topf mit Probenhalter, Blende und Schwingquarz.

Das Vakuum wird durch zwei Turbomolekularpumpen (Pfeiffer), je eine am oberen und unteren Teil der Kammer, und eine Ionengetterpumpe (Varian Starcell 300) erzeugt. Nach einem mehrtägigen Ausheizzyklus mit einer Höchsttemperatur von etwa $220^{\circ} \mathrm{C}$ wird ein Enddruck von etwa $5 \times 10^{-10}$ mbar erreicht.

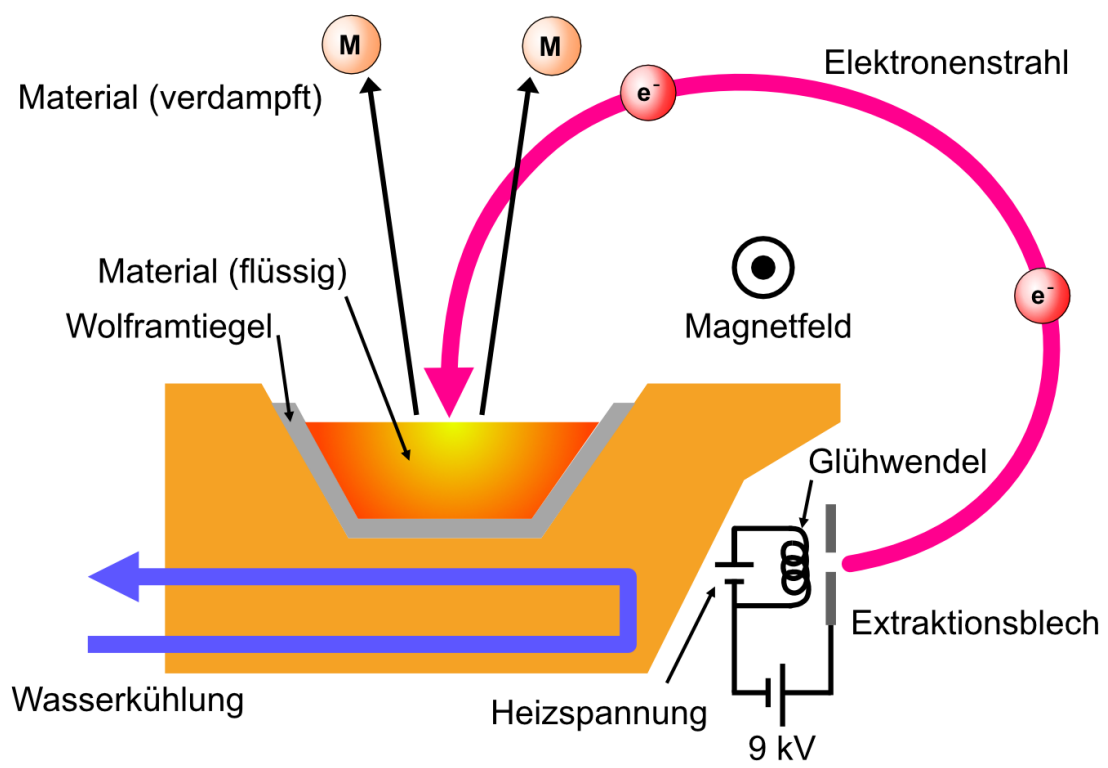

Abbildung 3.3: Funktionsprinzip eines Elektronenstrahlverdampfers.

Die Elektronenstrahlverdampfer sind zwei Ferrotec (AP\&T) und ein älteres Modell eines unbekannten Herstellers. Die Ferrotec Verdampfer haben eine Handsteuerung und die Möglichkeit, den Elektronenstrahl über zwei Achsen (x und y) über den Tiegel zu bewegen. Der ältere Verdampfer erlaubt nur ein Verschieben des Elektronenstrahls entlang der Hauptachse des Verdampfers.

Das technische Prinzip des Elektronenstrahlverdampfens ist in der Abbildung 3.3 dargestellt: Aus einer geheizten Glühwendel werden durch Anlegen einer Beschleunigungsspannung von mehreren Kilovolt gegen ein Extraktionsblech Elektronen herausgelöst und beschleunigt. Ein Stabmagnet im Inneren des Verdampfers (in der Skizze bis auf das Magnetfeld nicht eingezeichnet) lenkt die Elektronen auf eine 
Kreisbahn und in den Tiegel. Im Fall von Kupfer als Verdampfungsmaterial besteht der Tiegel aus Wolfram, um Material und den wassergekühlten Kupferkörper des Verdampfers thermisch zu trennen. Durch den Leistungseintrag des Elektronenstrahls erhitzt sich das Material im Tiegel und schmilzt. Von der Schmelze in die Vakuumkammer evaporierter Dampf fliegt quasi ballistisch und setzt sich auf allen Oberflächen, darunter Schwingquarz und Probe, ab. Über den Schwingquarz lässt sich die Aufdampfrate überwachen und mittels einer Leistungssteuerung des Elektronenstrahls nachregeln.

Der von mir genutzte Beschichtungsprozess ist im Anhang A.4 als Anleitung skizziert.

\subsection{Numerische Simulationen}

In Kapitel 2.2.1 haben wir mit der Rytov-Gleichung eine elegante, analytische Lösung zur Berechnung des Spektrums longitudinaler akustischer Schallwellen einer unendlich ausgedehnten Multilage hergeleitet. Um ein Verständnis für die im optischen Experiment verborgene Dynamik der Probe zu erhalten, sind solche analytischen Lösungen nur bedingt geeignet. Wie wir an dem Beispiel der Multilage mit Deckschicht gesehen haben, werden die Lösungen schnell sehr komplex, wenn überhaupt eine analytische Lösung für das aufgestellte System existiert.

Numerische Verfahren, wie zum Beispiel die Finite-Differenzen-Simulation (FDTD), sind hier ein guter Ersatz: Sie können auch für komplexere Systeme in durchführbarer Zeit eine hinreichende Näherungslösung der Systemdynamik finden. Bei geeigneter Wahl der Diskretisierungen der Simulation kann sogar eine Liveansicht der Probendynamik während der Simulation gerendert werden ${ }^{2}$. Hinzu kommt die relativ einfache Manipulation des simulierten Systems, zum Beispiel durch das systematische Auslenken bestimmter Simulationszellen.

Die während meiner Promotion genutzte FDTD-Simulation ist als Teil meiner Masterarbeit entstanden und in dieser ausführlich beschrieben [64]. An dieser Stelle verzichte ich daher auf eine erneute Beschreibung dieser Methode und meiner Masterarbeit sowie der zugehörigen Publikation [63].

${ }^{2}$ Dies ist von Vorteil, denn die Zwischenergebnisse der Simulation können direkt geprüft und ggf. vorzeitig abgebrochen und mit angepassten Parametern neu gestartet werden. 


\subsubsection{Born-von-Kármán Simulation}

In Kapitel 2.2.3 habe ich bereits die Grundlagen des BKM vorgestellt. An dieser Stelle möchte ich kurz die Umsetzung der Gleichung (2.32) als Simulation in ein funktionierendes Programm und die damit verbundenen Probleme skizzieren. Eine Kurzübersicht zum Benutzerinterface der Simulation ist im Anhang A.5 zu finden.

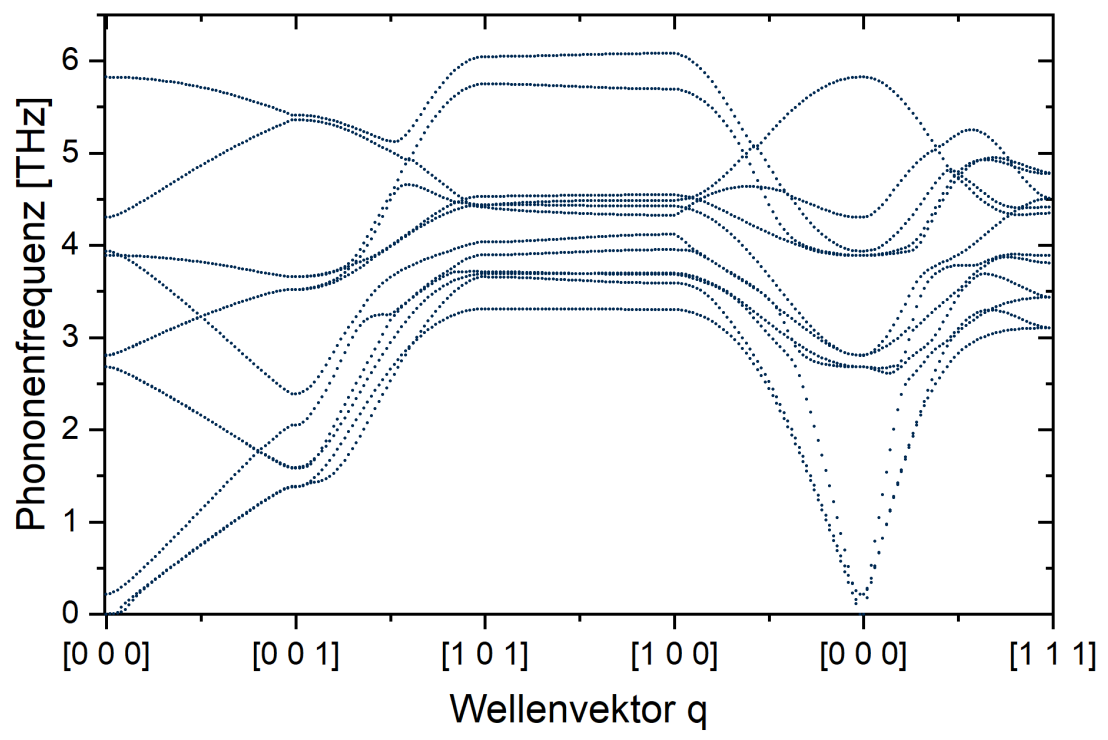

Abbildung 3.4: Schnitt entlang ausgezeichneter Symmetrieachsen durch die Brillouinzone eines $\mathrm{LMO}_{2} / \mathrm{SMO}_{2}$-Übergitters berechnet mit unserem BK-Modell. Weitere Spektren sind im Anhang A.6 aufgeführt.

In Abbildung 3.4 ist die so berechnete, akustische Bandstruktur eines $\mathrm{LMO}_{2} / \mathrm{SMO}_{2}{ }^{-}$ Übergitters mit rein kubischer Gitterstruktur zu sehen. Die Daten im Diagramm sind natürlich nur Schnitte entlang von Symmetrieachsen der 1. Brillouin-Zone (BZ) durch die Daten. Die tatsächliche Datenmenge beträgt $N^{3} \cdot 3 \cdot(m+n)$ Datenpunkte mit dem Datenvektor $\left(q_{x}, q_{y}, q_{z}, \omega\right)$ für jeden der $3 \cdot(m+n)$ Schwingungsäste und den q-Raum-Koordinaten $q_{x}, q_{y}$ und $q_{z}$. Die Simulationsdaten in Abbildung 3.4 umfassen also 1 Mio. Datenpunkte.

Eine Herausforderung bei der numerischen Berechnung der Bandstruktur ist das Sortieren der Eigenwerte zu zusammenhängenden Bändern. Aus der Berechnung selbst erhalten wir aufsteigend nach Größe sortierte Eigenwerte und damit Unste- 
tigkeiten an den Schnittpunkten von zwei oder mehreren Bändern. Für die Berechnung der Wärmeleitfähigkeit aus der Bandstruktur nach Gleichung (2.28) müssen wir die Phononengruppengeschwindigkeit bestimmen. Bei unsortierten Bändern würden diese unphysikalischen Unstetigkeiten in der Bandstruktur zu erhöhten Gruppengeschwindigkeiten und damit zu überhöhten Werten der Wärmeleitfähigkeit führen. Ein Zuordnen der Eigenwerte zu einzelnen, zusammenhängenden Bändern ist daher wichtig. Bei meinen Recherchen bin ich jedoch auf keine anwendbare Lösung für diese Aufgabe gestoßen. Aus diesem Grund habe ich einen Satz an parametrischen Sortieralgorithmen entwickelt, der Eigenwerte auf die einzelnen Phononenbänder aufteilen kann. Die Parameter im Benutzerinterface dienen zum Feineinstellen dieses Algorithmus und sind im Anhang A.5 genauer beschrieben.

Im ersten Schritt werden die Eigenwerte zunächst ohne eigentliches Sortieren auf Bänder aufgeteilt ${ }^{3}$. Von hier aus gehen wir dann in $q_{z}$ Richtung durch jedes Band, bestimmen den Gradient der letzten Punkte in z-Richtung und führen hiermit eine lineare Interpolation des nächsten Punktes durch. Von diesem Punkt aus suchen wir auf allen unsortierten Bändern den nächstgelegenen Punkt und sortieren ihn auf das aktuelle Band um. Dieses Vorgehen ist auf den ersten Blick für den Schnitt entlang der Hochsymmetrieachsen der 1. BZ ausreichend. Die dreidimensionale Darstellung der Bandstruktur zeigt jedoch, dass es abseits der Symmetrieachsen noch zu Sprüngen und Kanten in $q_{x}$ und $q_{y}$ kommt.

Ein dritter Schritt des Sortieralgorithmus basiert daher auf einem Bewertungssystem der Eigenwerte. Für jeden Eigenwert wird zu jedem Band eine Punktzahl berechnet. Der Eigenwert mit der jeweils höchsten Punktzahl wird auf ein bestimmtes Band umsortiert. Die Punkte stammen aus verschiedenen Bewertungsalgorithmen, dazu gehört z.B. die lineare Interpolation und Vergleich der Werte in je x, y und z Richtung, eine Bewertung für die direkte Nähe von einem Eigenwert zum nächsten, bereits einsortierten Punkt auf einem Band und ein Punktabzug, wenn das Einsortieren eines Wertes zu einer Unstetigkeit auf dem Band führt. Die Punkte für einzelne Kriterien können für jede Richtung individuell in der Benutzeroberfläche eingestellt werden. Die Standardwerte haben sich als guter Kompromiss für alle Modelle herausgestellt. Kleinere Unstetigkeiten, vor allem in Modellen mit vielen

${ }^{3}$ Als Bandindex eines Eigenwertes diente seine Position im Lösungsvektor des Eigenwertproblems. 
Bändern, lassen sich jedoch nur schwer vermeiden (siehe z.B. Sprünge in Abbildung 3.4). Die verbleibenden Sprünge treten gehäuft an den Zonengrenzen auf. Bereiche mit hoher Gruppengeschwindigkeit, welche den größten Beitrag zur Wärmeleitfähigkeit liefern, sind weitgehend sprungfrei. Wird eine ausreichend hohe Zahl $N$ an Punkten je Raumrichtung berechnet, wird der Beitrag von falsch einsortierten Eigenwerten klein gegenüber dem Beitrag der restlichen Bandstruktur. Dies ist z.B. dadurch zu erkennen, dass beim Erhöhen der Auflösung von $N$ die berechnete Wärmeleitfähigkeit aus der Bandstruktur schnell zu einem konstanten Wert konvergiert. $N=50$ hat sich als guter Kompromiss zwischen Berechnungsaufwand und Konvergenz von $\kappa$ herausgestellt und wurde für alle Berechnungen in dieser Arbeit verwendet.

\subsection{Pump-Probe-Reflektometrie (PPR)}

Die Pump-Probe-Reflektometrie (PPR) ist eine gängige Methode zur Untersuchung von Dynamik in dünnen Schichten, die sich auf der Piko- bis Nanosekundenzeitskala abspielen [68]. Für die PPR-Messungen in dieser Arbeit kamen mehrere verschiedene optische Aufbauten zum Einsatz, die sich in ihrer Funktionsweise weitgehend gleichen: Ein Femtosekundenlaser erzeugt einen Laserpuls, der an einem Strahlteiler meist in zwei unterschiedlich starke Teilpulse getrennt wird. Wir nennen die Pulse Probe-Puls (mit weniger Energie) und Pump-Puls (mit mehr Energie). Die Pulse durchlaufen gegebenenfalls verschiedene optische Elemente zur Modulation, Kollimation, Abschwächung, Polarisierung oder Frequenzverdoppelung für Zwei-Farb-Experimente und werden anschließend auf eine Probe gelenkt. Die Reflextion des Pump-Pulses wird in der Regel in einem Strahlblocker absorbiert, während die Reflexion des Probepulses über eine Photodiode in ein elektrisches Signal umgewandelt wird.

In einem der beiden Strahlwege wird eine motorisierte Schiene (Delaystage) mit einem Retroreflektor (oder zwei Spiegeln) verbaut, über welche die Weglänge dieses Strahlarms variiert werden kann. Wird nun diese Delaystage verfahren, verlängert oder verkürzt sich die Weglänge des einen relativ zum anderen Puls. Bei einer Verlängerung der Probe-Strecke verlängert sich folglich auch die Laufzeit des Pulses. Dieser kommt somit nach dem Pump-Puls an der Probe an. Trifft der energie- 
reiche Pump-Puls auf die Probe, wird ein Teil des Lichtes transmittiert, ein Teil reflektiert und der restliche Anteil absorbiert. Zur Anregung von akustischer Pikosekundendynamik spielt vor allem der absorbierte Teil des Pump-Pulses eine Rolle: Die Probenoberfläche wird erhitzt und das Material dehnt sich aus. Dies wiederum verursacht eine mechanische Verspannung in der Probe, die als mechanische Welle durch die Probe läuft.

a)

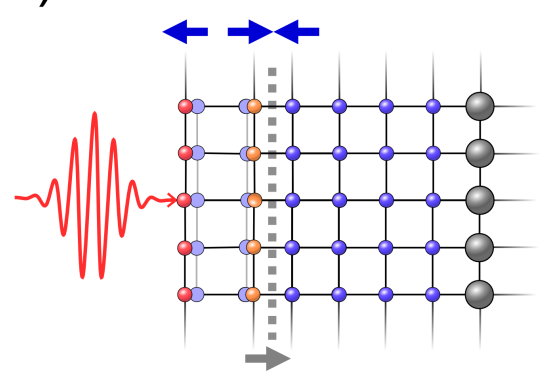

b)

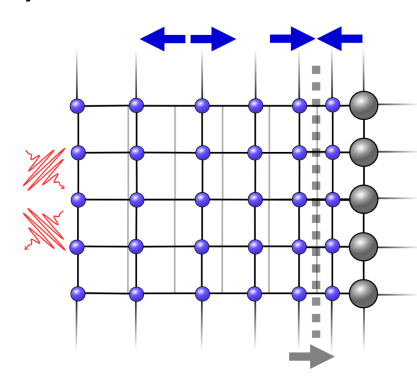

c)

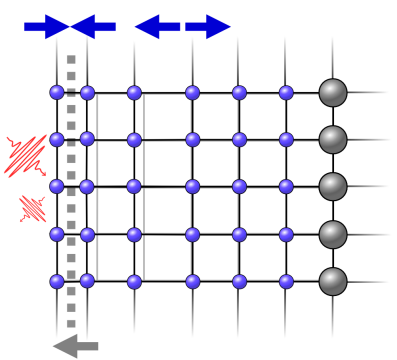

Abbildung 3.5: Vereinfachte Darstellung der Anregung und Messung von akustischen Wellen mit Hilfe des PPR-Setups. a) Intensiver Pump-Puls regt das Gitter an, welches sich ausdehnt und mechanische Spannung erzeugt. b) Die Verspannung des Gitters bewegt sich als Puls durch das Gitter und wird an einer akustischen Grenzfläche (teilweise) reflektiert. Ein Probepuls an der Oberfläche detektiert die Änderung der Verspannung als Änderung der Reflektivität. c) Der Puls erreicht die Oberfläche (und wird an der Grenzfläche zurückgeworfen). Ein Probepuls an der Oberfläche detektiert die Änderung der Verspannung als Änderung der Reflektivität.

Im Folgenden möchte ich die auftretende Dynamik nach Matsuda et al. [68] und Ruello et al. [89] skizzieren (siehe auch Abb. 3.5). Trifft ein ultrakurzer Laserpuls ein metallisches Probensystem, wird ein Teil seiner Energie im Elektronensystem absorbiert. Das angeregte Elektronensystem thermalisiert dann in wenigen Picosekunden (abhängig von der Elektron-Phonon-Kopplung des Materials) mit dem Atomgitter. Mit der Thermalisierung zwischen Elektronen und Gitter dehnt sich letzteres durch die Erhöhung der Gittertemperatur aus. Der mechanische Druck dieser lokalen Deformation läuft als kompakter Schallpuls vom Auftreffpunkt des Lasers aus in die Probe. Aufgrund der vielfältigen Wechselwirkungen zwischen 
Licht, Elektronen und Gitter ist die Berechnung des Spektrums dieses Pulses sehr komplex [90]. Aus Experimenten und Berechnungen ist bekannt, dass der akustische Puls durch fs-Laseranregung typischerweise Schwingungsfrequenzen im Bereich $10-1000 \mathrm{GHz}$ [68] enthält.

Reflexion und Transmission hängen mit dem Brechungsindex $n$ eines Festkörpers zusammen, welcher wiederum im Allgemeinen abhängig von Temperatur und mechanischer Spannung des Festkörpers ist. Für die Reflektivität des Films können wir

$$
\Delta R \propto \int_{0}^{\infty} d z\left(\frac{\partial n}{\partial T} \Delta T(z)+\frac{\partial n}{\partial \sigma} \Delta \sigma(z)\right) E^{2}
$$

schreiben [64, 68, 91]. Hier ist $E$ das elektrische Feld des Probestrahls, $T(z)$ die Temperatur und $\sigma(z)$ die mechanische Spannung als Funktion der Probentiefe in $z$-Richtung senkrecht zur Oberfläche. Für den Fall, dass der Probe-Puls zeitlich vor dem Pump-Puls auf der Probe ankommt, ist sein reflektiertes Signal noch unbeeinflusst von möglichen Effekten. Voraussetzung dafür ist, dass zwischen zwei Anregungen genug Zeit vergeht, dass die Probe wieder in ihren Ursprungszustand zurückkehren kann. Trifft der Probe-Puls zeitlich nach dem Pump-Puls auf die Probe, bewirken Änderungen der Probentemperatur und der mechanischen Verspannung der Probe eine Änderung in der Reflektivität. Diese Änderungen sind im Vergleich zur Gesamtreflektivität $R$ klein [68], weshalb im Experiment Pumpoder Probe-Puls mit Hilfe eines Choppers oder elektrooptischen Modulators zeitlich moduliert wird. Dem Photodetektor wird ein Lock-In-Verstärker nachgeschaltet, welcher das Messignal demoduliert und so die kleine Reflektivitätsänderung $\Delta R(t, \Delta \sigma, \Delta T)$ sichtbar macht. Die Dynamikmessungen an $\mathrm{W} / \mathrm{MgO} / \mathrm{ZrO}_{2}$ Multilagen wurden im PPR-Setup der Arbeitsgruppe von Prof. Dr. Münzenberg an der Universität Greifswald durchgeführt. Ein Ti:Sa fs-Laser erzeugt einen 60 fs Puls, welcher an einem Strahlteiler in einen Pump- und einen Probe-Puls aufgeteilt wurde. Der Pump-Puls wurde für jede Messung auf eine Leistung von $130 \mathrm{~mW}$ vor der Probe eingestellt. Auf der Probe wurde der Pump-Puls auf eine Halbwertsbreite von $60 \mu \mathrm{m}$ und der Probepuls auf eine Halbwertsbreite $30 \mu \mathrm{m}$ mittels des Verfahrens der Spots über eine Probenkante justiert. Der Überlapp der beiden Strahlen erfolgte mit Hilfe einer CCD-Kamera und der Maximierung des Messsignals. Zur Messung kommt ein Lock-In-Verstärker (SR830) und ein Chopper (Modulation 


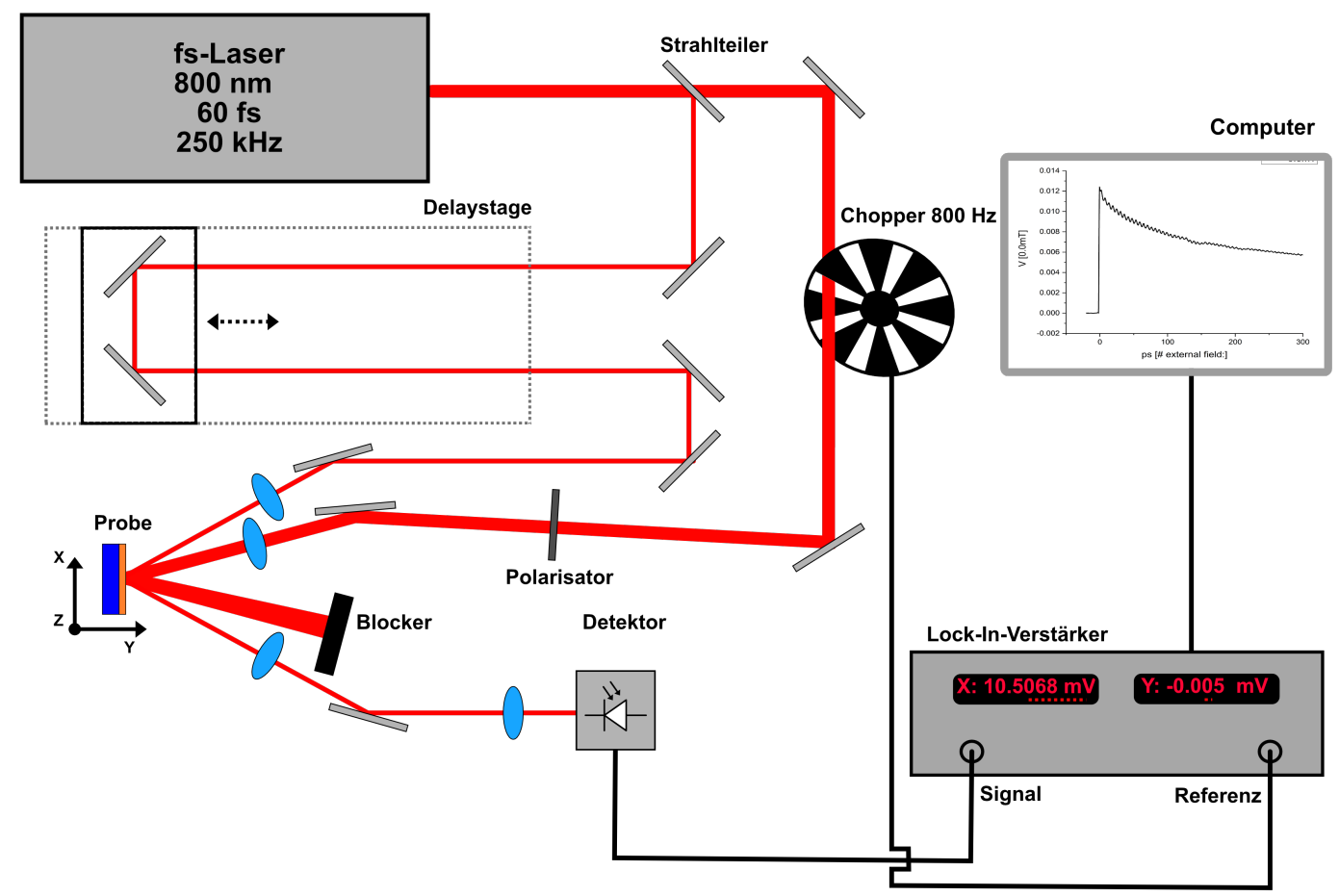

Abbildung 3.6: Schematischer Aufbau eines PPR-Experimentes.

des Strahls mit $800 \mathrm{~Hz}$ ) im Pumpstrahl zum Einsatz. Der Aufbau ist in Abbildung 3.6 vereinfacht dargestellt.

\subsection{Messmethode zur Wärmeleitfähigkeitsmessung}

Eine gängige Methode zur Bestimmung der Wärmeleitfähigkeit ist die sogenannte 3-Omega-Methode [92-94]. Diese Methode erfordert das Aufbringen einer elektrisch von der Probe isolierten Metallschicht, welche über optische Lithographie in eine Heizstruktur lithographiert werden muss. Die Messung der Wärmeleitfähigkeit erfolgt über eine gepulste Messung des Heizstroms mittels Lock-In-Messung und macht eine aufwendige Auswertung erforderlich [94].

Eine andere etablierte Methode zur Bestimmung der Wärmeleitfähigkeit von dünnen Schichten ist die sogenannte Time-Domain-Thermoreflektivität (TDTR). Bei dieser kontaktlosen Methode wird über die temperaturabhängige Reflektivität einer Probe die Temperaturänderung nach der Anregung mit einem Laser-Pump- 
Puls gemessen. Über einen zweiten, zeitverzögerten Probe-Puls wird die temperaturabhängige Reflektivität der Probe gemessen [95]. Besonders metallische Filme zeigen eine hohe Abhängigkeit der Reflektivität von der Temperatur [96, 97]. Tatsächlich kennen wir die Messsignatur der Probenoberflächentemperatur bereits aus den PPR-Messungen aus Kapitel 3.3. Für die elastische ps-Dynamik ziehen wir den thermischen Hintergrund jedoch normalerweise von den Messungen ab.

Aufgrund des Funktionsprinzips von Pump-Probe-Experimenten mit fs-Lasern sind wir bei der Zeitauflösung der Thermoreflektivitätsmessungen jedoch auf wenige Nanosekunden limitiert, genauer auf die Lichtlaufzeit über die mechanischen Verzögerungsstrecken (Delaystage). Der Wärmetransport in mehrere Nanometer dicken Schichten findet jedoch typischerweise [86, 97] auf Zeitskalen von hunderten Nanosekunden oder Mikrosekunden statt. Eine mechanische Verzögerungsstrecke müsste für solche Experimente mehrere hundert Meter lang sein. Eine Alternative dazu ist die sogenannte Transiente Thermoreflektivität (TTR): Ähnlich wie bei TDTR heizt ein Pump-Puls die Probenoberfläche auf. Anstatt einer stroboskopischen Messung wird jedoch ein CW-Laser kontinuierlich von der Probe reflektiert. Die Intensität des reflektierten Lichtes kann elektronisch mit einem Oszilloskop oder einer schnellen Messkarte aufgenommen werden, was bei einem Oszilloskop mit einer Abtastrate von $500 \mathrm{MHz}$ eine Zeitaufösung ab 2 ns erlaubt. Zusammen erlauben TDTR und TTR die rein optische Messung der Temperaturdynamik einer Probe, auf den Zeitskalen von wenigen fs bis einige Sekunden.

Im Gegensatz zur 3-Omega-Methode erfordert das TTR keine Lithographie der Probe und funktioniert auf isolierenden sowie auf elektrisch leitfähigen Proben (z.B. Lanthanstrontiummanganat). Damit ist es in Hinblick auf die Probenauswahl deutlich flexibler und weniger aufwendig in der Probenpräparation [97].

\subsubsection{Transiente Thermoreflektivität (TTR)}

In der ersten Förderperiode des SFB 1073 wurde ein solcher TTR-Messaufbau in der Arbeitsgruppe von Prof. Dr. Krebs im Rahmen des Projektes A02 aufgebaut und für erste TTR-Messungen genutzt [86, 91, 97]. Als Quelle für den Heizpuls wurde hier ein Excimer-Laser genutzt, welcher auch für die PLD-Beschichtung verwendet wurde (siehe Kapitel 3.1.2). Die Reflektivität der Proben wurde zeitabhängig mit einem $638 \mathrm{~nm}$ Diodenlaser der Firma Toptica, einer schnellen Pho- 
todiode (100 MHz, Firma Femto) und einem Oszilloskop gemessen. Die Proben wurden mit metallischen Filmen beschichtet. Diese Filme dienen zum einen dazu, einen definierten Bereich zu schaffen, in dem der Laserpuls seine thermische Energie deponieren kann. Zum anderen haben viele Metalle eine starke Abhängigkeit des Reflextionskoeffizienten von der Schichttemperatur [98] und dienen somit auch als Thermometer für die Probe. Für die Wellenlänge des Probelasers als besonders geeignet hat sich eine Deckschicht aus $50 \mathrm{~nm}$ Kupfer herausgestellt [97, 98].

Bei meinem Eintritt in das Projekt mit Beginn der 2. Förderperiode des SFB wurde der Excimer-Laser außer Dienst gestellt und das TTR-Experiment abgebaut, weshalb wir uns entschieden haben, einen neuen TTR-Messaufbau in unserem Labor aufzubauen. Details zur Entwicklung dieses Aufbaus sind im Anhang A.3.1 beschrieben.

\subsubsection{TTR-Aufbau}

In Abbildung 3.7 ist die aktuell neuste Revision unseres TTR-Experimentes skizziert. Mit diesem Aufbau wurden sämtliche TTR-Messungen, welche ich in dieser Arbeit zeige, gemessen. Der $515 \mathrm{~nm}$ Heizpuls stammt aus einem diodengepumpten Festkörperlaser der Firma Coherent (FlareNX 515). Die Repetitionsrate beträgt $2000 \mathrm{~Hz}$ und die Pulslänge laut Datenblatt 1.3(2) ns. Wir haben uns speziell wegen der hohen Repetitionsrate für die Anschaffung dieses Lasers entschieden. Die Pulsenergie am Laser beträgt $260 \mu \mathrm{J}$ (Leistung $520 \mathrm{~mW}$ und die Halbwertsbreite auf der Probe zwischen $1 \mathrm{~mm}$ bis $3 \mathrm{~mm}$. Damit sind wir, vor allem wenn noch fokussierende Optiken zum Einsatz kommen sollten, bei Energiedichten, wie sie bei der Laserdeposition zum Verdampfen von Kupfer verwendet werden [97]. Bei einer Halbwertsbreite von $<0.3 \mathrm{~mm}$ wäre die Energiedichte etwa $>0.7 \mathrm{~J} \mathrm{~cm}^{-2}$. Im Vergleich ist die Energiedichte für PLD von Kupfer im Bereich $1 \mathrm{~J} \mathrm{~cm}^{-2}$ bis $5 \mathrm{~J} \mathrm{~cm}^{-2}$ [91]. Aus diesem Grund können wir im Pumpstrahlengang auf fokussierende Optiken verzichten und um Schäden an der Probenoberfläche auszuschließen schwächen wir mit einem ND-Filter die Strahlleistung ab.

Vom Pumpstrahl wird an einem 9:1 Strahlteiler ein kleiner Teil des Pulses auf einen Photodetektor umgelenkt, welcher das Triggersignal für das Oszilloskop liefert. Die restliche Leistung vom Strahl wird über eine Anordnung von Spiegeln auf die 


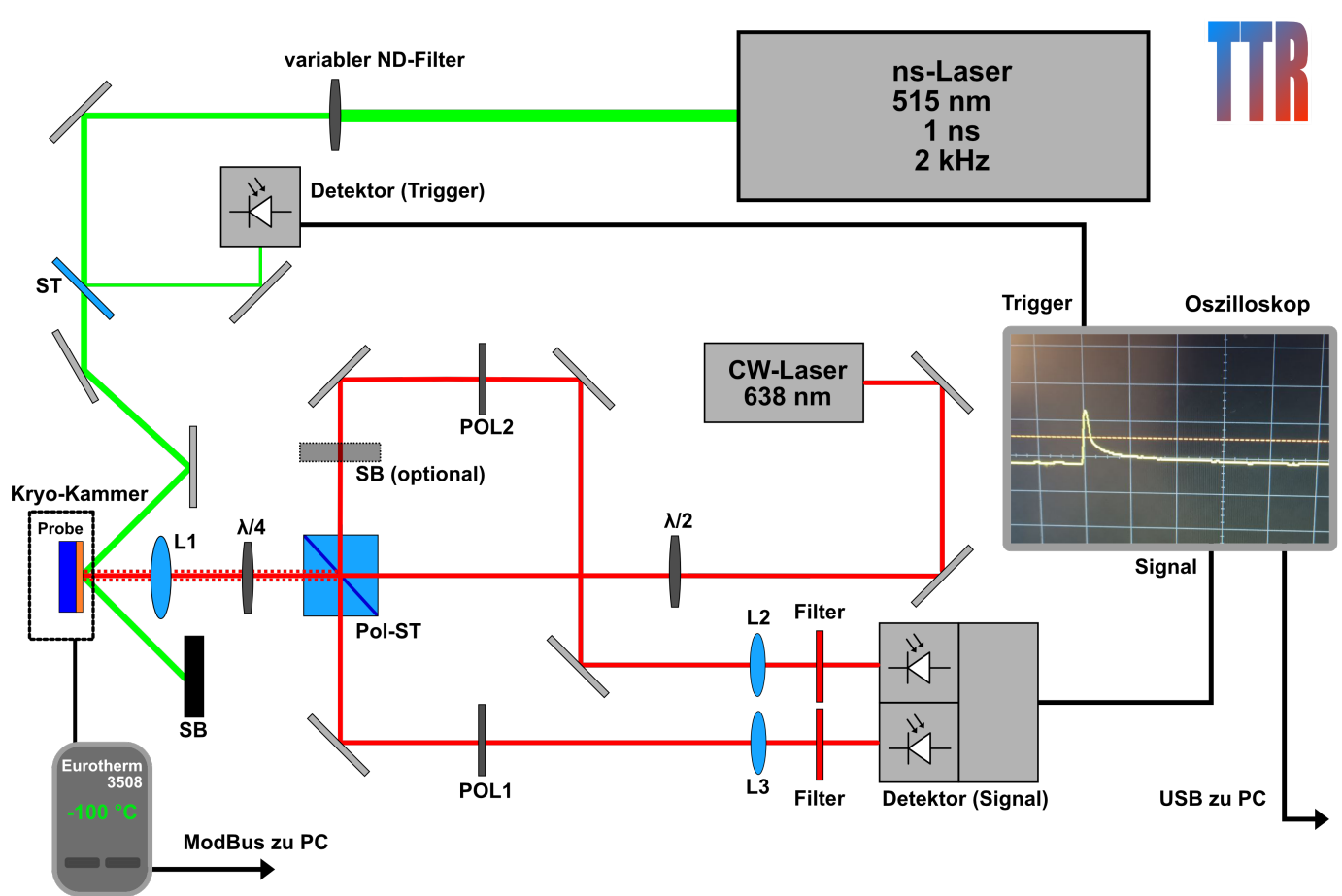

Abbildung 3.7: Skizze des TTR-Versuchs.

Probe gelenkt. Die Reflexion des Pulses von der Probe wird in einem eloxierten Aluminiumblech absorbiert.

Der Probestrahl wird von einem temperaturstabilisierten CW-Diodenlaser mit maximal $250 \mathrm{~mW}$ Leistung erzeugt. Über eine $\lambda / 2$-Platte und einen polarisierenden Strahlteiler (Pol-ST) kann die transmittierte Leistung geregelt werden ${ }^{4}$. Der an Pol-ST 90 reflektierte Teil des Strahls kann wahlweise als Referenzstrahl für den symmetrischen Detektor verwendet werden oder in einem Strahlblocker absorbiert werden. In letzterem Fall muss der Referenzeingang des Photodetektors blockiert werden.

Der an Pol-ST transmittierte Strahl durchläuft eine $\lambda / 4$-Verzögerungsplatte, welche so eingestellt ist, dass der Strahl zirkular polarisiert wird. Über die Objektivlinse L1 wird der Probe-Strahl auf die Probe fokussiert und die Rückreflexion in einen rückläufigen Strahl gebündelt. Der Durchmesser des Probe-Strahls auf der Probe

${ }^{4}$ Der Diodenlaser ist in seiner Leistung per PC einstellbar, doch hat er bei $>50 \mathrm{~mW}$ eine stabile Laseremission. 
beträgt etwa $23 \mu \mathrm{m}$. Beim erneuten Durchlaufen der Verzögerungsplatte wird die Polarisation um $90^{\circ}$ zum einlaufenden Strahl gedreht, woraufhin er am Strahlteiler in den unteren Arm zum Photodetektor reflektiert wird. Über die Polarisatoren POL1 und POL2 lassen sich Probestrahl und Referenzstrahl abschwächen, um ein Überladen der Photodioden zu verhindern. Die Linsen L2 und L3 fokussieren das Licht auf den jeweiligen Eingang des Photodetektors. Das intensive Streulicht des Pump-Lasers wird von zwei Bandpassfiltern (640 nm zentrale Wellenlänge) daran gehindert, in die Eingänge des Detektors zu gelangen.

\subsubsection{Auswertung von TTR-Messdaten}

Das Ziel einer TTR-Messung ist es, die Wärmeleitfähigkeit einer Schicht zwischen Kupferfilm und Substrat zu bestimmen. Die Reflektivität der Kupferoberfläche der Probe folgt in erster Ordnung [96]

$$
\frac{\Delta R}{R}=\frac{1}{R} \frac{\partial R}{\partial T} \Delta T=C \Delta T
$$

mit dem Thermoreflexionskoeffizient $C$. Für Kupfer liegt der absolute Wert laut Literatur bei etwa $C_{\mathrm{Cu}} \approx-8,5 \cdot 10^{-5} \mathrm{~K}^{-1}$ [98]. Trifft der Pulslaser auf den Kupferfilm, wird er von diesem teilweise absorbiert und heizt den Film auf. Die Absorption des 515 nm-Pump-Pulses beträgt bei $45^{\circ}$ Einfallswinkel etwa $50 \%$ [67]. Die absorbierte Energie beträgt damit $E_{\text {absorb. }}=0.5 \cdot 50 \mathrm{~mW} / 2 \mathrm{kHz}=12.5 \mu \mathrm{J}$. Die thermische Eindringtiefe [99]

$$
d_{\mathrm{Th} .}=\sqrt{\frac{\kappa}{\rho C_{\mathrm{p}}} \tau}
$$

ist eine Abschätzung für die Ausdehnung des Temperatureinflusses eines Laserpulses, der in einem Material absorbiert wird. Mit der Wärmeleitfähigkeit $\kappa$, Dichte $\varrho$ und spezifischer Wärme $C_{\mathrm{p}}$ von Kupfer (siehe Tabelle A.1) und der Pulsdauer $\tau=1.3 \mathrm{~ns}$ beträgt sie etwa $d_{\mathrm{Th}} \approx 500 \mathrm{~nm}$. Dies ist eine Größenordnung über der Dicke der Kupferschicht von $d_{\mathrm{Cu}}=50 \mathrm{~nm}$, weshalb wir die Absorption der Laserenergie in der Kupferschicht als gleichmäßig annehmen können. Die Temperaturerhöhung des Kupfers durch den Laserpuls ist dann in einer vereinfachten Abschätzung:

$$
\Delta T=\frac{E_{\text {absorb. }}}{\varrho \cdot d_{\mathrm{Cu}} \pi r^{2} C_{\mathrm{P}}} .
$$


Nehmen wir einen Strahlradius von etwa $r=1 \mathrm{~mm}$ an ergibt sich eine Temperaturänderung im Kupfer von $\Delta T \approx 23 \mathrm{~K}$. Diese Änderung ist in einem initialen Abfall der Reflektivität (Gleichung (3.2)) von $0.25 \%$ zu beobachten. Bei einer Photodiodenspannung entspricht dies einer Änderung von etwa $3 \mathrm{mV}$ auf dem Oszilloskop.

Unter der Annahme, dass die Breite des Pump-Pulses auf der Probenoberfläche deutlich größer ist, als die Breite des Probestrahls und die Gesamtdicke der zu untersuchenden Schicht, können wir im Anschluss an den Pump-Puls von einem eindimensionalem Wärmetransport senkrecht zur Oberfläche ausgehen.

Nach dem initialen Aufheizen der Probenoberfläche fließt nun Wärmeenergie über die zu untersuchende Schicht in das Substrat ab. Ist die Wärmeleitfähigkeit der Schicht klein gegenüber Kupfer und Substrat bildet sie einen „Flaschenhals“ für den Wärmetransport und dominiert das Abklingverhalten der Reflektivität. In seiner Dissertation leitet Florian Döring [97] nach Carslaw und Jaeger [100] für den Fall einer Kupferschicht auf einer Bulkprobe aus der allgemeinen Wärmeleitungsgleichung

$$
\frac{\partial T(x, t)}{\partial t}=\frac{\kappa}{\rho c} \frac{\partial^{2} T(x, t)}{\partial x^{2}}
$$

eine analytische Lösung für die Temperatur der Kupferoberfläche her. Sie lautet:

$$
T(z=0, t)=\int_{0}^{t} \theta\left(t^{\prime}\right) \cdot T\left(z=0, t-t^{\prime}\right) d t^{\prime}=\frac{2 Q}{\tau e \sqrt{\pi}}(\sqrt{t}-\sqrt{t-\tau}) .
$$

$\theta(t)$ ist eine Funktion zur Beschreibung des (als Rechteckpuls angenommenen) Laserpuls, $Q$ die gesamte Wärmeenergie, welche der Puls auf die Probe aufbringt und $\tau$ die Pulslänge. $e=\sqrt{\kappa \rho \cdot c}$ ist die thermische Effusivität des Materials unter der Kupferschicht. In der Herleitung dieser Gleichung wurde das Material unter dem Kupfer als unendlich dick angenommen. Dies ist für die Auswertung von Messungen an sehr dicken Bulkproben (z.B. Kupferfilm direkt auf Substraten oder Bulkschichten) sicherlich eine akzeptable Näherung. Für Systeme aus Kupferfilm, Schicht und Substrat stellt F.D. die Bedingung auf, dass für die Dicke $L$ der Schicht

$$
L>\sqrt{\frac{10 \kappa}{\rho c} \tau}
$$


gelten muss [97], also die thermische Eindringtiefe klein im Vergleich zur Schichtdicke ist. Es lässt sich schnell erkennen, dass für viele interessante Materialien die Schicht mehrere hundert Nanometer dick sein muss, z.B. für $\mathrm{SrMnO}_{3}$ gilt nach dieser Formel $L>135 \mathrm{~nm}\left(\kappa_{\mathrm{SMO}}=4.5 \mathrm{~W} \mathrm{~m}^{-1} \mathrm{~K}^{-1}\right.$ [101], Dichte $\rho_{\mathrm{SMO}}=5500 \mathrm{~kg} \mathrm{~m}^{-3}$ [101], Spezifische Wärme $c_{\mathrm{SMO}}=544 \mathrm{~J} \mathrm{~kg}^{-1} \mathrm{~K}^{-1}$ [81], $\left.\tau=1.2 \mathrm{~ns}\right)$.

Sind die zu untersuchenden Schichten dünner, beeinflusst die Wärmeleitung im Substrat und die Absorption des Heizpulses im Kupfer immer stärker das Abklingverhalten der Temperatur im Kupfer und unabhängige Werte für $\kappa$ lassen sich durch Anpassen von Gl. (3.6) nicht mehr aus den Messdaten extrahieren.

Eine Lösung für dieses Problem ist es, den Beitrag der Wärmeleitung aus allen Schichten der Proben in der Herleitung der Oberflächentemperatur zu berücksichtigen. In einer Arbeit von Balageas et al. [102] wird der Fall eines Drei-SchichtSystems beschrieben. In unserer Arbeitsgruppe haben wir jedoch eine allgemeine Formulierung des Wärmetransportes nach dem Beispiel von Balageas et al. für ein System aus n-Schichten aufgestellt welche zusätzlich die Grenzfächenleitfähigkeiten zwischen diesen Schichten beinhaltet. Die Herleitung dieses Modells möchte ich hier als Grundlage unserer TTR-Auswertungsprogramme kurz skizzieren.

Ausgehend von Gleichung (1.2) definieren wir für die i-te Schicht von insgesamt $N$ Schichten die Wärmediffusiongleichung

$$
\frac{\partial T_{i}(z, t)}{\partial t}=\alpha_{i} \frac{\partial^{2} T_{i}(z, t)}{\partial z^{2}}
$$

mit der Zusammenfassung der materialspezifischen Parameter zu $\alpha=\frac{\kappa}{\rho c}$. An den Übergängen von Schicht $i$ zu Schicht $i+1$ (am Ort $z_{i}$ ) führen wir die Grenzflächenleitfähigkeit $h_{i}\left(\right.$ Einheit $\left.\mathrm{W} \mathrm{m}^{-2} \mathrm{~K}^{-1}\right)$ ein. Mit

$$
-\kappa_{i} \frac{\partial T_{i}\left(z_{i}, t\right)}{\partial z}=-\kappa_{i+1} \frac{\partial T_{i+1}\left(z_{i}, t\right)}{\partial z}=h_{i}\left[T_{i}\left(z_{i}, t\right)-T_{i+1}\left(z_{i}, t\right)\right]
$$

stellt diese Randbedingung die Energieerhaltung an den Grenzflächen sicher. Da wir von nur geringen Temperaturen im Bereich von Raumtemperatur ausgehen, vernachlässigen wir den Wärmeverlust durch Abstrahlung von der Kupferoberfläche und der Rückseite des Substrats. Für die Grenzflächen an Proben Ober- und Unterseiten legen wir die Grenzflächenleitfähigkeiten daher auf $h_{0}=h_{N}=0$ fest. 
Eine Wärmeabgabe an die Umgebung wird in unserem Modell somit vernachlässigt. Zusätzlich modellieren wir die Anregung durch den Laser als Delta-Funktion zum Zeitpunkt $t=0$. Die Randbedingungen sind zusammengefasst also

$$
\begin{aligned}
\text { Oberseite } z=0: & -\kappa_{1} \frac{\partial T_{1}(0, t)}{\partial z}=\delta(t) \\
\text { Unterseite } z=z_{N}: & -\kappa_{N} \frac{\partial T_{N}\left(z_{N}, t\right)}{\partial z}=0 \\
\text { Grenzflächen } z_{i}: & -\kappa_{i} \frac{\partial T_{i}\left(z_{i}, t\right)}{\partial z}=-\kappa_{i+1} \frac{\partial T_{i+1}\left(z_{i}, t\right)}{\partial z}=h_{i}\left[T_{i}\left(z_{i}, t\right)-T_{i+1}\left(z_{i}, t\right)\right]
\end{aligned}
$$

Bevor das gesamte Gleichungssystem aufgestellt wird, transformieren wir Diffusionsgleichung und Randbedingungen zunächst mittels einer Laplace-Transformation in den Laplace-Raum. Durch diesen Schritt vereinfachen sich die zeitlichen Ableitungen zu Produkten aus Laplacefaktor $s$ und der transformierten Temperatur $\tilde{T}$.

Wir erhalten folgende Diffusionsgleichung und Randbedingungen im Laplace-Raum:

Diffusionsgleichung : $\quad s \tilde{T}_{i}(z, s)=\alpha_{i} \frac{\partial^{2} \tilde{T}_{i}(z, s)}{\partial z^{2}}$

$$
\begin{aligned}
\text { Oberseite } z=0: & -\kappa_{1} \frac{\partial T_{1}(0, s)}{\partial z}=1 \\
\text { Unterseite } z=z_{N}: & -\kappa_{N} \frac{\partial \tilde{T}_{N}\left(z_{N}, s\right)}{\partial z}=0 \\
\text { Grenzflächen } z_{i}: & -\kappa_{i} \frac{\partial \tilde{T}_{i}\left(z_{i}, s\right)}{\partial z}=-\kappa_{i+1} \frac{\partial \tilde{T}_{i+1}\left(z_{i}, s\right)}{\partial z}=h_{i}\left[\tilde{T}_{i}\left(z_{i}, s\right)-\tilde{T}_{i+1}\left(z_{i}, s\right)\right]
\end{aligned}
$$

Die transformierte Diffusionsgleichung wird von

$$
\tilde{T}_{i}(z, s)=X_{i}(s) e^{ \pm \sqrt{\frac{s}{\alpha_{i}}} z}
$$

gelöst. Eine allgemeine Lösung ist somit die Linearkombination

$$
\tilde{T}_{i}(z, s)=X_{i}(s) e^{+\sqrt{\frac{s}{\alpha_{i}}} z}+Y_{i}(s) e^{-\sqrt{\frac{s}{\alpha_{i}}} z}
$$


mit den durch die Randbedingung bestimmten Amplituden $X_{i}(s)$ und $Y_{i}(s)$. Analog zu meiner Herleitung der Rytov-Gleichung (Gleichung (A.8) im Anhang) können wir diesen Ausdruck durch trigonometrische Funktionen darstellen als

$$
\tilde{T}_{i}(z, s)=A_{i}(s) \sinh \left(\sqrt{\frac{s}{\alpha_{i}}} z\right)+B_{i}(s) \cosh \left(\sqrt{\frac{s}{\alpha_{i}}} z\right)
$$

mit $A_{i}(s)=X_{i}(z)+Y_{i}(z)$ und $B_{i}(s)=X_{i}(z)-Y_{i}(z)$. Zusammen mit den vorher genannten Randbedingungen erhalten wir ein Gleichungssystem aus 2N Gleichungen, welches in der Matrixform $M$ als

$$
M \cdot\left(A_{1}, B_{1}, \ldots, A_{i}, B_{i}, \ldots, A_{N}, B_{N}\right)^{T}=(1,0, \ldots, 0)^{T}
$$

dargestellt werden kann. Durch Einsetzen von $z=0$ für den Ort der Probenoberflächen in Gleichung (3.14) finden wir $\tilde{T}_{1}(0, s)=\tilde{T}_{\text {Oberfl. }}(s)=B_{1}(s) . B_{1}(s)$ stellt also die von uns gesuchte Oberflächentemperatur im Laplace-Raum mit der Spektralkoordinate $s$ dar. Die Oberflächentemperatur $\tilde{T}_{\text {Oberfl. }}(s)$ lässt sich nun mit Hilfe der Cramerschen Regel und dem Laplaceschen Entwicklungssatz berechnen. Für $\tilde{T}_{\text {Oberfl. }}(s)$ folgt

$$
\tilde{T}_{\text {Oberfl. }}(s)=\frac{\operatorname{det} M_{12}^{\downarrow}}{\operatorname{det} M}
$$

$M_{12}^{\downarrow}$ ist die Matrix $M$ mit der gestrichenen ersten Reihe und der zweiten Spalte durch den Lösungsvektor aus Gl. (3.15) ersetzt. Um diesen analytischen Ausdruck an die TTR-Messdaten anpassen zu können, müssen wir $\tilde{T}_{\text {Oberfl. }}(s)$ zunächst wieder aus dem Laplace-Raum in den Zeitraum zurück transformieren:

$$
T_{\text {Oberfl. }}(t)=\frac{1}{2 \pi i} \lim _{x \rightarrow \infty} \int_{\xi-i x}^{\xi+i x} \tilde{T}_{\text {Oberfl. }}(s) e^{(s t)} d s .
$$

$\xi$ muss dafür so gewählt werden, dass es größer ist als alle realen Polstellen in $\tilde{T}$. Das Aufösen des Integrals kann über den Residuuensatz erfolgen [80]. Wir erhalten letztendlich die zeitabhängige Evolution der Oberflächentemperatur:

$$
T_{\text {Oberfl. }}(t)=T_{\mathrm{GW}}+\sum_{k=1}^{\infty}\left(\frac{\operatorname{det} M_{12}^{\downarrow}}{\partial \operatorname{det} M / \partial s}\right) e^{\left(s_{k} t\right)} .
$$


Hier sind $s_{k}$ die Polstellen in Gl. (3.16), also Punkte, an denen $\operatorname{det} M=0$ gilt. $T_{\mathrm{GW}}=1 / \sum_{i}^{N} \rho_{i} c_{i} d_{i}$ ist der Wert des Residuums bei $s_{0}=0$ und stellt die Gleichgewichtstemperatur für $t \rightarrow \infty$ dar.

Gleichung (3.18) lässt sich mit Hilfe der Matrixelemente von $M_{12}^{\downarrow}$ vergleichsweise unkompliziert in Programmcode übersetzen. Angepasst an unsere typischen Proben aus Kupferschicht, Übergitter und Substrat wählen wir $N=3$, also eine $6 \mathrm{x} 6$ Matrix mit 17 nicht verschwindenden Termen. Die größte Herausforderung hierbei ist die Summe von $k=1$ bis $\infty$, für welche wir eine sinnvolle obere Grenze finden müssen: Damit $e^{\left(s_{k} t\right)}$ nur zeitlich abklingende Summanden besitzt, können wir ein oberes Limit für $k$ finden: Die spektrale Auflösungsgrenze unseres Setups liegt durch die Laserpulslänge von 1 ns und der Wahl des Photodetektors bei etwa $500 \mathrm{MHz}$. Folglich reicht es zum Anpassen von $T_{\text {Oberfl. }}(t)$ an die Messdaten aus, alle Summanden mit $\left|s_{k}\right|<500 \mathrm{MHz}$ zu berücksichtigen. Die Beiträge von $\left|s_{k}\right|<500 \mathrm{MHz}$ zu $T_{\text {Oberfl. }}(t>2 \mathrm{~ns})$ können vernachlässigt werden, da dieser Bereich durch das Experiment nicht abgebildet wird. Aus praktischen Gründen wählen wir generell $k_{\max }=2000$.

Das Fitprogramm wurde von mir in C\# geschrieben. Mit Hilfe der frei verfügbaren Bibliothek MathNet lassen sich sämtliche (komplexen) Matrizen und Vektoren einfach eingeben. Die implementierten Gleichungen wurden speziell an unsere Proben mit $N=3$ an unser typisches Probensystem aus Kupfer, zu untersuchende Schicht (Übergitter, PMMA, usw.) und Substrat angepasst. Die Nullstellen Suche erfolgt über das Brent-Suchverfahren welches in MathNet integriert ist. Für die Wahl der Startwerte für das Suchverfahren verwende ich einen einfachen Algorithmus, der verhindern soll, dass das Brent-Verfahren zu bereits berechneten Nullstellen konvergiert und eventuell Nullstellen bei zu großem Testintervall überspringt. Dies ist im Folgenden als Pseudocode skizziert: 


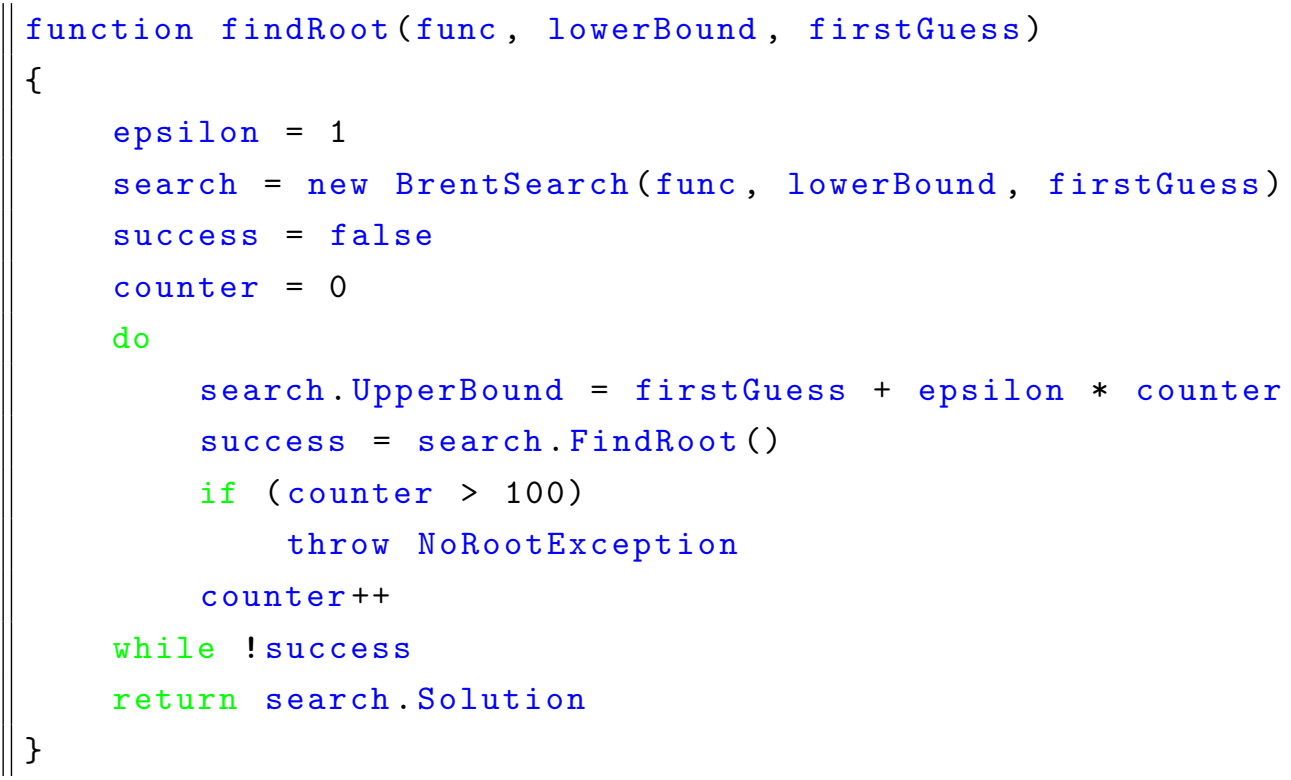

Vergleiche mit anderen Bibliotheken und Programmen zur Nullstellensuche haben gezeigt, dass diese Methode im direkten Vergleich sehr schnell ist und dank dem größer werdenden Testintervall weder Nullstellen überspringt noch zurück zu bekannten Nullstellen springt. Unter Ausnutzung der Tatsache, dass die Nullstellen in ähnlich großen Schritten verteilt sind, lässt sich die Nullstellensuche weiter beschleunigen, indem wir eine sinnvolle Startposition der nächsten Nullstelle raten. Hierzu definieren wir $\Delta s=s_{i-1}-s_{i-2}$ als die Differenz der Position der letzten beiden Nullstellen. Die Position der nächsten Nullstelle schätzen wir als $s_{i, \text { Guess }}=s_{i-1}+\Delta s$ ab und übergeben diese an die Funktion findRoot. Die untere Grenze (lowerBound) für die Suche wählen wir als $s_{i, \text { Start }}=s_{i-1}+0.1$, die obere Grenze der Suche wird dynamisch angepasst. Es gilt $s_{i \text {,Ende }}=s_{i \text {, Guess }}+j \cdot 1$. Wir lassen dabei j von 1 bis 100 laufen, solange das MathNet Brent-Suchverfahren keine Nullstelle findet. Typischerweise ist eine Nullstelle bereits bei $j<5$ gefunden. Die zusätzlichen Durchgänge sind für Spezialfälle von extremen Fit-Parametern eingebaut, z.B. in Vergleich zu den anderen Schichten sehr dünne Kupferfilme.

Die Kombination aus MathNet-Klassen und optimierter Nullstellensuche erlaubt die Berechnung einer TTR-Kurve in wenigen Millisekunden ${ }^{5}$ und ermöglicht damit ein Anpassen der Kurven an die Messdaten innerhalb weniger Minuten.

\footnotetext{
${ }^{5}$ Auf meinem Desktop-PC im Durchschnitt 12 ms pro 2000 Nullstellen.
} 
Das Berechnen der theoretischen TTR-Kurve ist in folgendem Pseudocode skizziert:

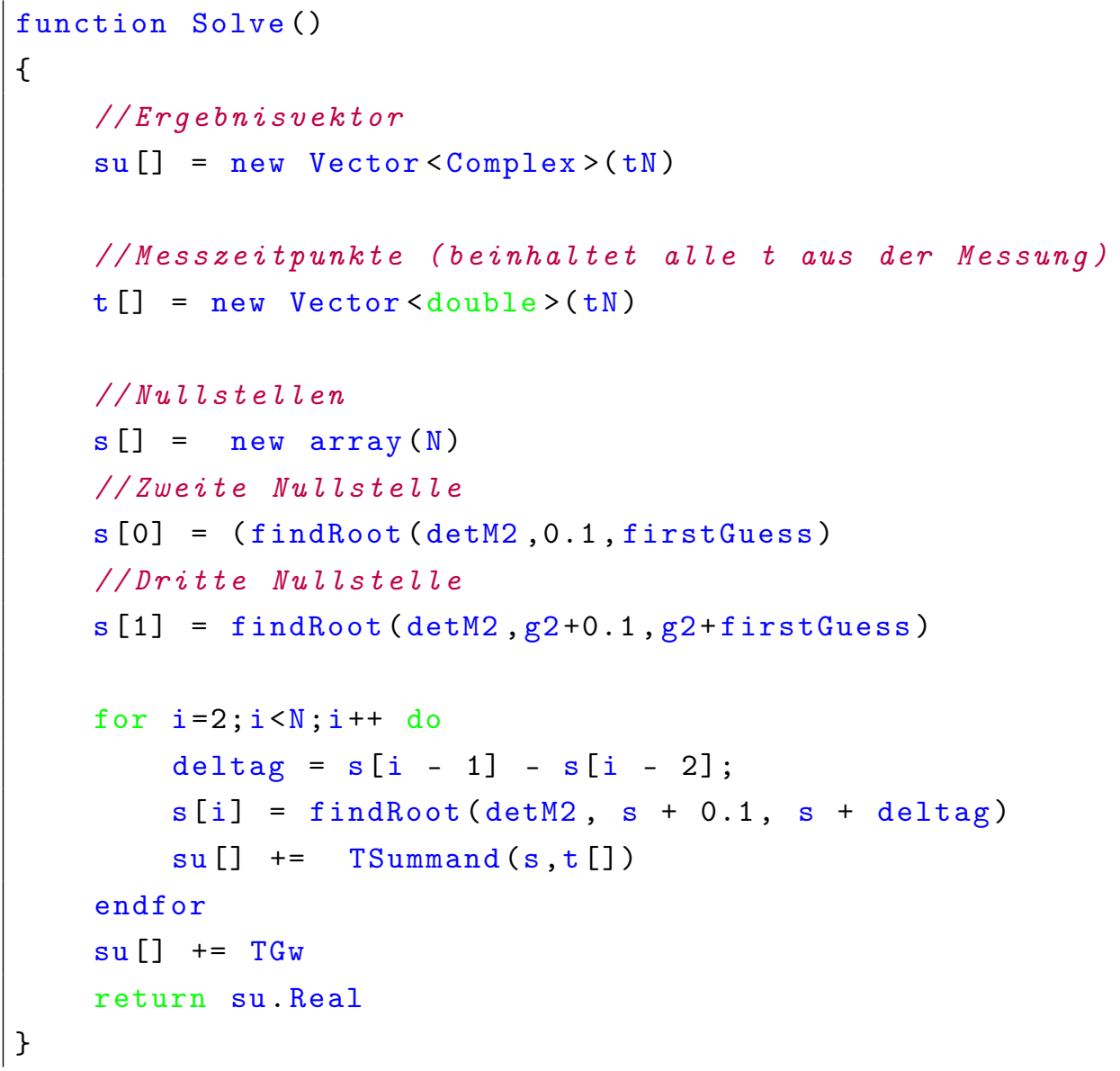

In diesem Codebeispiel ist tN die Anzahl der Messpunkte bzw. Zeitschritte, N die Anzahl der zu bestimmenden Nullstellen und detM2 die Determinante der Matrix $M_{2}$ (siehe Gl. (3.18)). su [] ist ein Vektor zum Speichern unserer Kurve und TSummand eine Funktion, welche den Summanden in Gleichung (3.18) als Vektor und über alle Elemente in $\mathrm{tN}$ abbildet.

Zum Anpassen haben wir uns für das Nelder-Mead-Verfahren [103] entschieden. Dieses Verfahren kommt ohne die (händische) Berechnung der Ableitung der zu minimierenden Funktion aus und ist in MathNet für eine beliebige Anzahl anzupassender Parameter implementiert. Im Programm nutzen wir dieses Nelder- 
Mead-Verfahren um die Parameter von $T_{\text {Oberfl. }}$ zu finden, welche das Fehlerquadrat zwischen berechneter Abklingkurve und Messwerten $T_{\text {Daten }}(t)$ minimiert:

$$
\operatorname{Min}\left\{\chi^{2}\right\}=\operatorname{Min}\left\{\sum_{t=0 \text { ns }}^{t_{\text {max. }}}\left(T_{\text {Daten }}(t)-T_{\text {Oberfl. }}(t)\right)^{2} / N\right\} \text {. }
$$

Die Gesamtreflektivität $R$ und der in erster Näherung lineare Zusammenhang zwischen Temperatur und relativer Reflektivitätsänderung (siehe Gl.(3.2)) können wir als konstante Faktoren vernachlässigen, wodurch wir die einfache Proportionalität $\Delta R \propto \Delta T$ erhalten. Somit können wir die Minimierung in Gl. (3.19) direkt auf die auf 1 normierte Spannungsänderung der Photodiode im TTR-Messaufbau übertragen. Im Programm können nun wahlweise die Wärmeleitfähigkeit $\kappa_{2}$, die spezifische Wärme $C_{2}$ oder die Grenzflächenwiderstände $h_{1}$ und $h_{2}$ oder eine beliebige Kombination aus diesen an diese Messdaten angepasst werden. Der Index bezieht sich dabei auf die zweite Schicht in unserem Dreischichtmodell.

Es wäre ohne weiteres möglich, das Programm darin zu erweitern, auch andere Parameter, wie beispielsweise die Materialparameter der anderen Schichten oder die Schichtdicken anzupassen. Da wir für unsere Messungen jedoch die Parameter von Substrat und Kupferschicht aus Literaturangaben heranziehen können und die Schichtdicken generell gut durch Röntgenreflektometrie bestimmt werden können, haben wir auf diese Funktion im Programm verzichtet.

In Abbildung 3.8 ist die Oberfläche des Auswertungsprogramms dargestellt. 


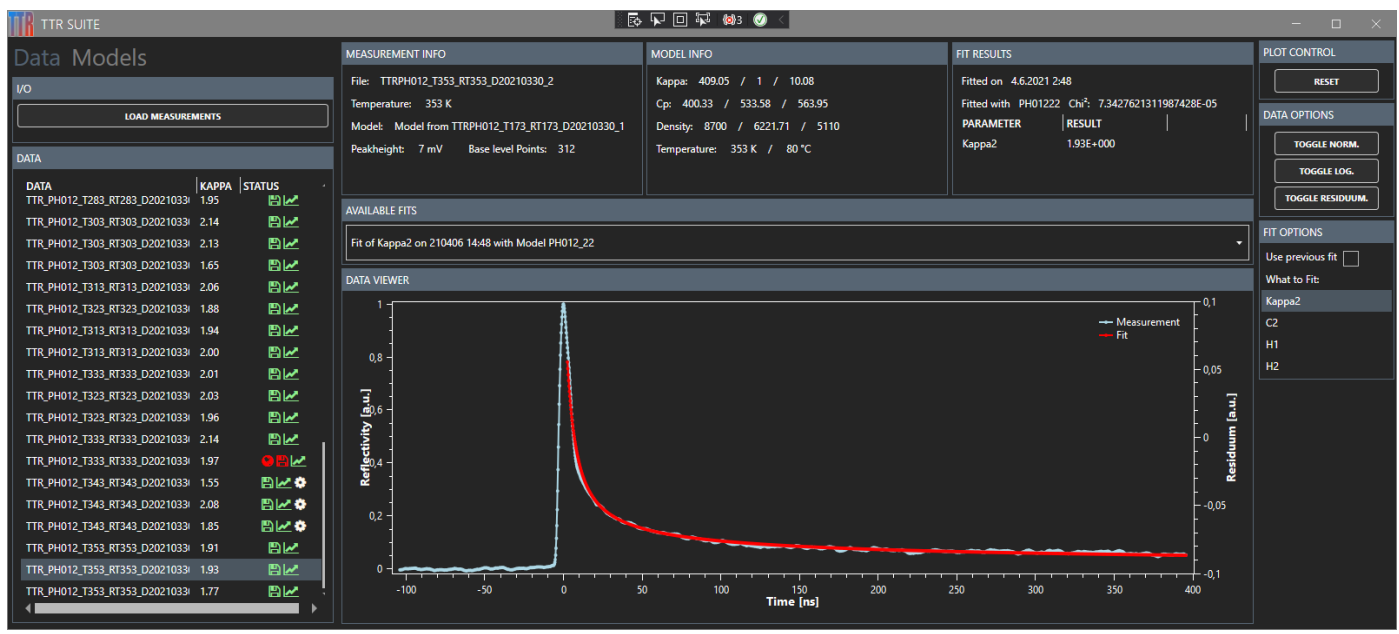

Abbildung 3.8: Die Oberfläche des Auswertungsprogramms für TTR-Messkurven.

Da der TTR-Aufbau mit dem neuen Laser sehr viele Einzelmessungen erzeugen kann (etwa 5 Minuten pro Messkurve mit großer Mittelung) und dies vor allem für temperaturabhängige Messungen schnell einen Datensatz von mehr als 50 einzelnen Messkurven pro Messtag generiert, war es mir wichtig, das Auswertungsprogramm so benutzerfreundlich und effizient wie möglich zu gestalten. Die Messdaten können per Dialog oder Drag-and-Drop direkt vom Messrechner in das Programm gezogen werden. Parameter wie Temperatur, Signalstärke und Anzahl der Messpunkte werden direkt aus den Messdaten ausgelesen. Die Messdaten werden außerdem beim Laden normiert und das Maximum der Reflektivitätsänderung wird auf den Zeitnullpunkt verschoben. Zum Anpassen der Kurven wird ein sogenanntes Model im Programm angelegt oder aus einer Datei geladen. In diesem Model können die Parameter der einzelnen Schichten und der Grenzflächen eingegeben werden. Für alle materialspezifischen Parameter lassen sich auch temperaturabhängige Datensätze laden. Diese Modelle können einzelnen oder allen Datensätzen gleichzeitig zugewiesen werden. Per Rechtsklick können dann die Kurven an die Messdaten angepasst werden, welche die Auswahl der freien Parameter minimiert.

Alle erzeugten Daten können im BSON-Format ${ }^{6}$ abgespeichert und später erneut in das Programm geladen werden. Über das Rechtsklickmenü bei der Auswahl mehrerer Daten mit angepasster Kurve kann ein Ergebnisfenster geöffnet werden. Aus diesem lassen sich alle angepassten Parameter z.B. als Funktion der Temperatur

\footnotetext{
${ }^{6}$ Das Binärformat des von Menschen lesbaren JSON-Format.
} 
exportieren. Mehrere Kurven an einer Temperatur lassen sich gemittelt und mit dem Fehler aus der Standardabweichung exportieren.

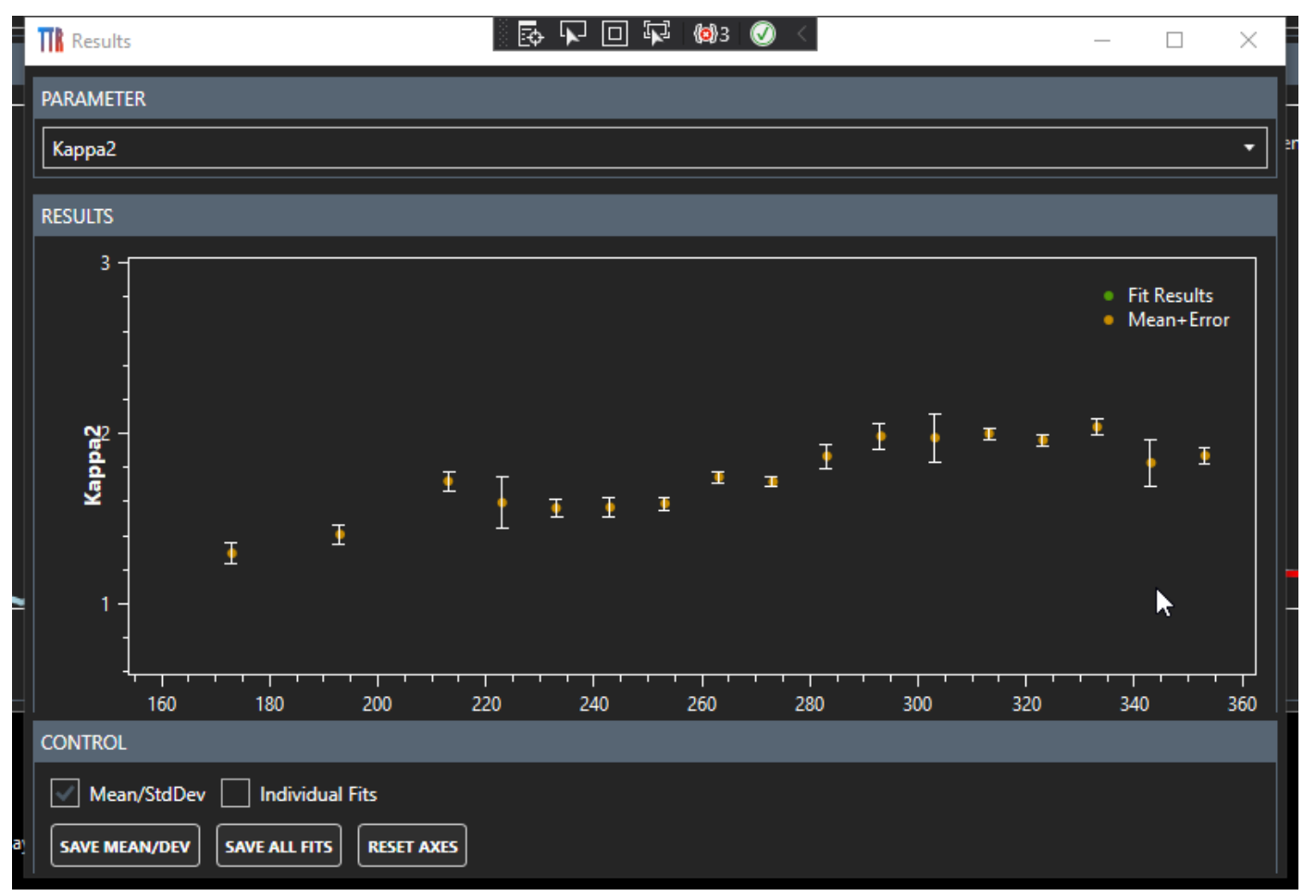

Abbildung 3.9: Ergebnisfenster für Datensätze mit erfolgreich angepasstem $\kappa$. Der Fehler ist die Standardabweichung der Mittelung aus mehreren Messkurven. 


\subsubsection{Temperaturabhängige Transiente Thermoreflektivität (TTTR)}

Für temperaturabhängige Messungen (temperaturabhängige transiente Thermoreflektivitiät (TTTR)) habe ich in Zusammenarbeit mit Kai Dörner einen Probenhalter mit einfachem Kryostat konstruiert. Dieser Probenhalter besteht aus einem Kupferturm mit einem eingefrästen Kanal, durch welchen flüssiger Stickstoff gepumpt werden kann. Am oberen Teil dieses Kupferturms sind zwei $40 \mathrm{~W}$ Heizpatronen neben einer Gewindebohrung eingesetzt. In diese Gewindebohrung wird ein kupferner Teller geschraubt, auf welchem die Probe und ein Pt1000Thermoelement festgeklemmt wird. Der Kupferturm ist über eine isolierende Keramik in einer kleinen Kammer auf einem X-Y-Z-Positionierer angebracht. Alle Kabel werden durch eine gedichtete Durchführung aus der Kammer herausgeführt. Die Stickstoffleitung wird über Teflonschläuche und einen Messingflansch herausgeführt. Zwischen Flansch und Kammer ist eine etwa $5 \mathrm{~cm}$ langer KF-Wellbalg, welcher die Durchführung thermisch von der Kammerwand trennt. Über den Turm kann eine eloxierte Haube mit Laserfenster aufgesetzt werden, dies erlaubt einen optischen Zugang zum Pump- und Probe-Laser. Die in Reihe geschalteten Heizpatronen werden über ein Netzteil (Delta Elektronika) mit Strom versorgt. Die Steuerung des Netzteils wird von einem Temperaturregler (Schneider Elektronik Eurotherm 3508) übernommen, welches über das Pt1000 die Temperatur des Probentellers misst. Mit Hilfe eines Pumpstands kann die Kammer auf etwa $5 \times 10^{-5} \mathrm{mbar}$ abgepumpt werden. Dadurch soll Kondensation von Wasser am Fenster und auf der Probe verhindert werden.

In Testläufen konnte ich den Probenteller durch Unterdruckpumpen von flüssigem Stickstoff aus einem Dewargefäß auf $100 \mathrm{~K}\left(-173^{\circ} \mathrm{C}\right)$ abkühlen und mit den Heizpatronen auf $373 \mathrm{~K}\left(100^{\circ} \mathrm{C}\right)$ erwärmen. Die maximale Temperatur liegt vermutlich noch höher, aus Rücksicht auf Dichtungen und Vakuumfett wurde jedoch nicht über $100^{\circ} \mathrm{C}$ geheizt.

Alle Schritte für eine TTR-Messung sind im Anhang A.3.2 als Messanleitung aufgeführt. 


\subsubsection{Evaluation des TTR-Aufbaus}

Um eine allgemeine Einschätzung der Leistungsfähigkeit unseres TTR-Experimentes zu bekommen, habe ich vier Proben aus je einer $50 \mathrm{~nm}$ Kupfer und einer Zirkondioxidschicht $\left(\mathrm{ZrO}_{2}\right)$ mit verschiedenen Dicken (50 nm, $200 \mathrm{~nm}, 500 \mathrm{~nm}$ und $1 \mathrm{\mu m}$ ) gemessen. Diese Proben wurden von Florian Döring mit Hilfe der PLD (siehe Kapitel 3.1.2) auf Siliziumoxid-Substrat hergestellt. Die Proben dienten bereits für die Gruppe von Prof. Dr. Krebs als Referenz- und Vergleichsproben, zur Entwicklung des dort implementierten TTR-Aufbaus [97].

(a)

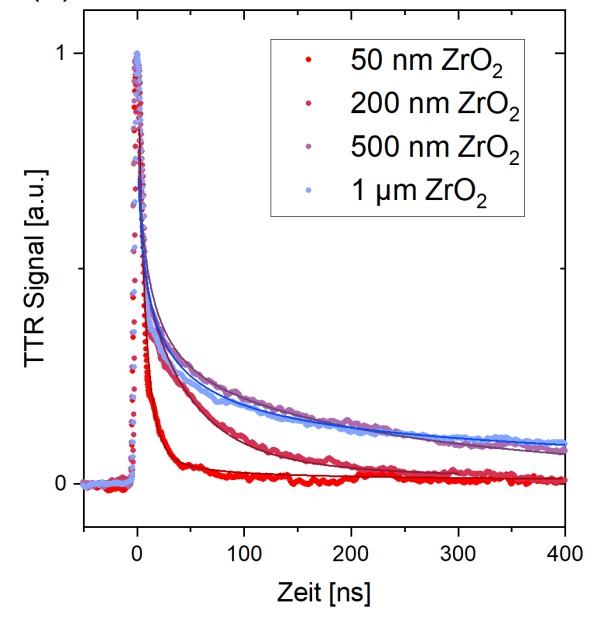

(b)

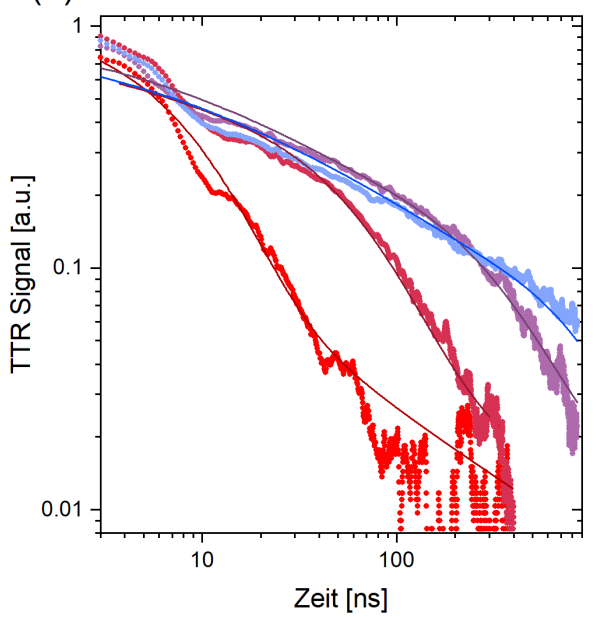

Abbildung 3.10: (a) Auf das Maximum normierte TTR-Messdaten für unterschiedliche Schichtdicken $\mathrm{ZrO}_{2}$. Alle Proben sind mit $50 \mathrm{~nm}$ Kupfer beschichtet. Die angepassten Kurven stammen aus unserem TTR-Auswertungsprogramm, welches in Kapitel 3.4.1.2 vorgestellt wurde. (b) Die selben Daten wie in (a) in doppelt logarithmischer Auftragung. Dies betont die gute Konvergenz der angepassten Kurven im Zeitbereich von 10 ns bis 500 ns.

An den Messungen in Abb. 3.10 (a) sehen wir gut, dass wir die Temperaturdynamik der Kupferschicht über die Reflektivität bereits grob ablesen können. Wie in der Beschreibung des Experimentes erwähnt, ist die Schicht zwischen der Kupferlage und dem Substrat bei hinreichend kleiner Wärmeleitfähigkeit $\left(\kappa_{\text {Schicht }} \ll \kappa_{\mathrm{Cu}}\right)$ der Flaschenhals des Wärmetransportes von der lasergeheizten Kupferschicht in das Substrat. Ist diese Schicht dünn, dann fließst die Wärme aus der Kupferschicht schneller ab, als wenn bei gleichem $\kappa_{\text {Schicht }}$ die Schicht dicker wäre. Dies sehen wir auch in den Messdaten, denn das Kupfer mit der dünnsten $\mathrm{ZrO}_{2}$ Schicht 
$\left(d_{\text {Schicht }}=50 \mathrm{~nm}\right)$ darunter benötigt nur etwa $100 \mathrm{~ns}$ um auf die Temperatur von vor dem Heizpuls (bei $t=0$ ) abzukühlen. Bei $d_{\text {Schicht }}=200 \mathrm{~nm}$ ist diese Zeit schon bei etwa $300 \mathrm{~ns}$ bis $400 \mathrm{~ns}$. Die noch dickeren $\mathrm{ZrO}_{2}$ fallen im gemessenen Zeitfenster gar nicht auf den Wert vor dem Anregen der Probe. Hier zeigt sich sehr gut, dass an der reinen TTR-Messung ein Abschätzen der Wärmeleitfähigkeit qualitativ möglich ist, aber für eine genauere Betrachtung diese Messkurven tiefgehender modelliert werden müssen, als nur durch das reine Anpassen eines Abklingkoeffizienten (vgl. Gleichung (3.6)). Die doppelt logarithmische Darstellung in Abb. 3.10 (b) verdeutlicht das komplizierte Verhalten der Oberflächentemperatur und offenbart unterschiedliche Bereiche:

- Von 0 ns bis 10 ns ist die Dynamik in allen Proben nahezu identisch. In der logarithmischen Auftragung in Abb. 3.10 (b) ist zu erkennen, dass das Modell diesen Bereich nur schlecht beschreibt. Durch den Laserpuls von etwa 1.2 ns Pulslänge, der Abtastrate des Oszilloskops von $500 \mathrm{MHz}$ und der Bandbreite von $100 \mathrm{MHz}$ ( $-3 \mathrm{~dB}$ Bandbreite) des Photodetektors sind wir für einzelne Messpunkte hier in einem Bereich, welcher unter der zeitlichen Auflösungsgrenze des Aufbaus liegt. Die Messdaten zeigen also den verwaschenen ns-Laserpuls, welcher die Probenoberfläche stark aufheizt.

- Ab 12 ns bzw. nachdem der direkte Einfluss des Pump-Pulses im Messsignal abgeklungen ist, wird die Temperaturänderung von der (niedrigen) Wärmeleitfähigkeit der Zwischenschicht dominiert.

- Auf langen Zeiten dominiert die Wärmeleitfähigkeit des Substrates. Dies ist im gewählten Messzeitraum nur für $d_{\text {Schicht }}=50 \mathrm{~nm}$ ab etwa $t=50 \mathrm{~ns} \mathrm{zu}$ beobachten.

In unserem Modell der Oberflächentemperatur (siehe Kapitel 3.4.1.2) berücksichtigen wir die zeitliche Breite des Pump-Pulses [102], vernachlässigen aufgrund der sehr hohen Wärmeleitfähigkeit aber das Absorptionsprofil im Inneren der Kupferschicht. Da dieser Abschnitt der Messdaten außerhalb der zeitlichen Auflösungsgrenze liegt, erwarten wir von dort auch keine Rückschlüsse auf die Wärmeleitfähigkeit der Zwischenschicht. Für das Anpassen des Modells können wir die ersten 10 ns vernachlässigen. Gleiches gilt für lange Zeitskalen. Hier dominieren die Eigenschaften des Substrats und der letzten Grenzfläche. Außerdem erreichen wir bei 
niedrigen Änderungen der Reflektivität das vertikale Auflösungsvermögen (Spannungsauflösung) des Oszilloskops.

Der Verlauf des Bereich zwischen 20 ns und 800 ns, welcher von der Wärmeleitfähigkeit der Zwischenschicht geprägt ist, wird von den angepassten Kurven gut erfasst. Die über $\chi^{2}$ minimierten Wärmeleitfähigkeiten der Zwischenschicht $\kappa_{\text {Schicht }}=\kappa_{\mathrm{ZrO}_{2}}$ sind in Abb. 3.11 gezeigt.

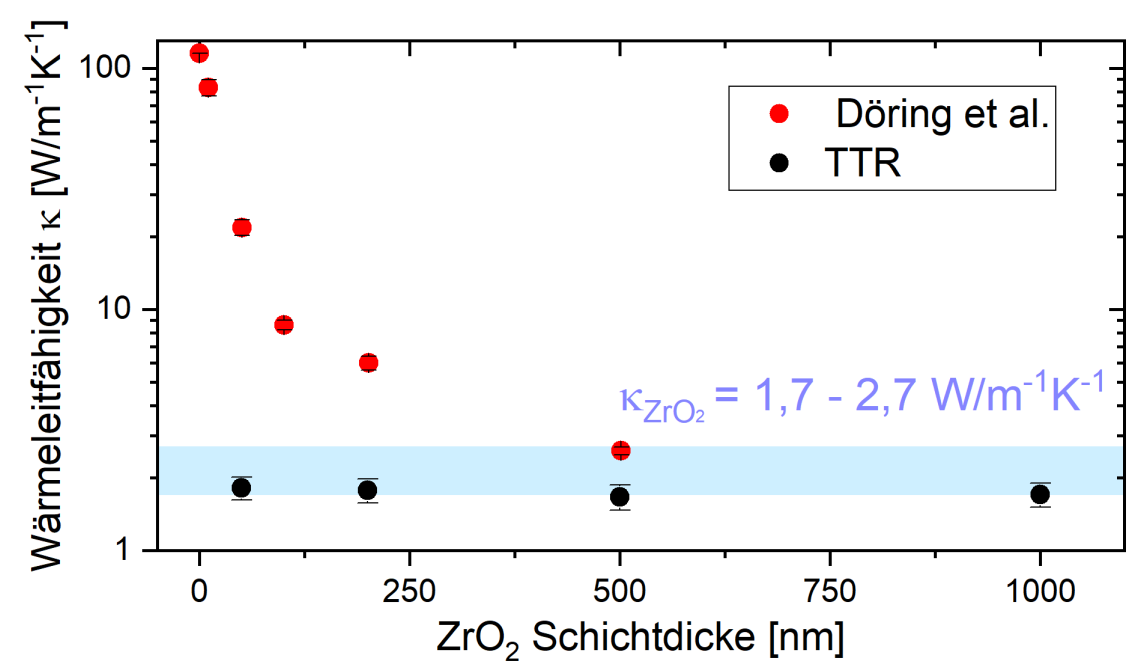

Abbildung 3.11: Vergleich von angepassten Wärmeleitfähigkeiten an $\mathrm{Cu} / \mathrm{ZrO}{ }_{2}$ Proben mit unserem Aufbau und unserer Modellierung (schwarz) und dem Aufbau und Auswertungsmethode aus der 1. Förderperiode (rot) [97]. Der Literaturwert für $\kappa_{\mathrm{ZrO}_{2}}$ [104] ist als Bereich (hellblau) eingezeichnet. Es handelt sich bei gleicher Schichtdicke um dieselben Proben. Die Messungen fanden bei Raumtemperatur statt.

Es ist gut zu erkennen, dass unsere TTR-Methode Literaturwerte für die Wärmeleitfähigkeit einer bekannten Schicht reproduzieren kann. Auch sehen wir, dass unsere Modellierung des Experimentes die komplexen Einflüsse von Kupferschicht, Grenzflächen und Substrat auf die zeitliche Entwicklung der Oberflächentemperatur gut modelliert und so die verschiedenen Einflüsse auf die zu bestimmende Wärmeleitfähigkeit reduziert. Im Gegensatz zur ersten Förderperiode des SFB 1073 können wir mit unserer Methode auch die Wärmeleitfähigkeit von dünnen Proben bestimmen. Dank den Verbesserungen am optischen Aufbau und der hohen Repe- 
titionsrate des neuen Lasers konnten wir außerdem das Signal-Rausch-Verhältnis verbessern. 



\section{Kapitel 4}

\section{Ergebnisse}

\subsection{Ultraschall in $\mathrm{W}\left[\mathrm{MgO} / \mathrm{ZrO}_{2}\right]$}

Zum Bestimmen der Schallgeschwindigkeit in Wolframfilmen habe ich mit Hilfe der EBPVD-UHV-Anlage vier dünne Wolframfilme der Dicken 20.8(8) nm, 30.0(8) nm, $45.0(8) \mathrm{nm}$ und 58.6(8) $\mathrm{nm}$ auf thermisch oxidiertem Siliziumsubstrat hergestellt und mit dem PPR-Aufbau die Oberflächendynamik untersucht. An diesen Messungen möchte ich sowohl die generellen Auswertungsschritte für die PPR-Messungen zeigen als auch die Schallgeschwindigkeit von Wolfram für die Interpretation späterer Messungen bestimmen. Die Frequenzen in Abb. 4.1 wurden aus dem Reflexionssignal (Inset in Abb. 4.1) bestimmt, indem an die Fouriertransformation des Signals eine Voigt-Funktion der Form

$$
y(f)=y_{0}+\frac{A \ln (2)}{\pi^{\frac{3}{2}}} \frac{w_{\mathrm{L}}}{w_{\mathrm{G}}^{2}} \int_{-\infty}^{\infty} \frac{e^{-t^{2}}}{\left(\frac{\sqrt{(\ln (2)} w_{\mathrm{L}}}{w_{\mathrm{G}}}\right)^{2}+\left(\frac{\sqrt{4 \cdot \ln (2)}\left(f-f_{0}\right)}{w_{\mathrm{G}}}-t\right)^{2}} \mathrm{~d} t
$$

angepasst wurde. Der Parameter $f_{0}$ ist dabei die Mitte dieser Voigt-Kurve und die mittlere Frequenz des FFT-Signals im Spektrum der Signal-Oszillation. An die so ermittelten schichtdickenabhängigen Resonanzfrequenzen wird nun die folgende Gleichung für die Resonanzfrequenz eines freien Wolframfilms selber Dicke angepasst:

$$
f\left(v_{\mathrm{W}}, d_{\mathrm{W}}\right)=\frac{v_{\mathrm{W}}}{2 d_{\mathrm{W}}} .
$$

Hier ist $v_{\mathrm{W}}$ die gesuchte Schallgeschwindigkeit des Wolframfilms und $d_{\mathrm{W}}$ seine über Röntgenreflektometrie bestimmte Schichtdicke. Aus der Anpassung finden wir für 
(a)

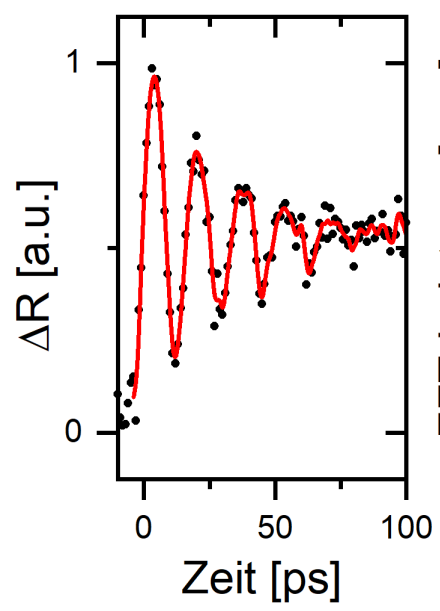

(b)

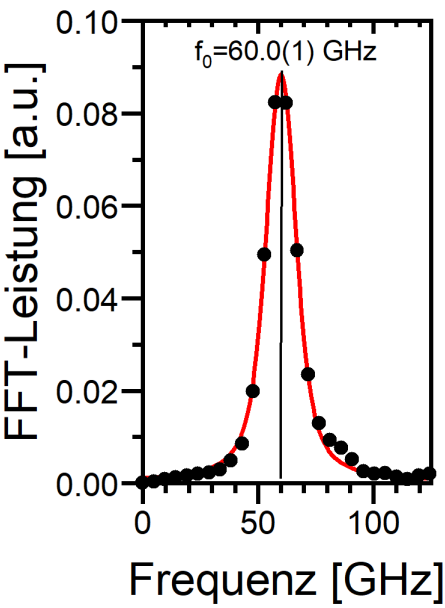

(c)

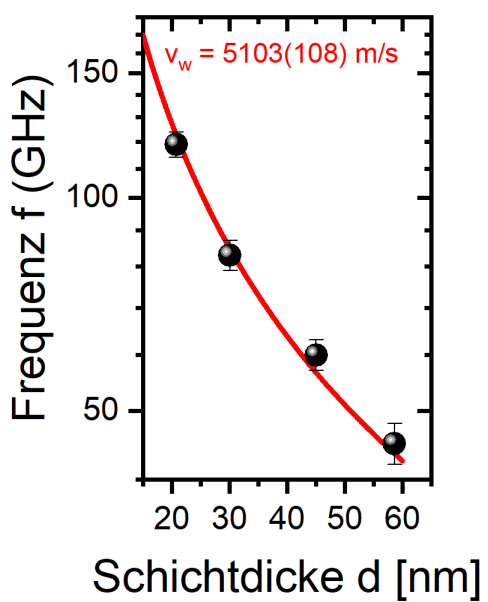

Abbildung 4.1: (a) PPR-Messsignal an einer $45 \mathrm{~nm}$ dicken Wolframschicht auf Siliziumdioxid-Substrat. Die rote Linie ist eine Glättung der Messdaten und dient als Sichthilfe. (b) FFT der Messkurve aus (a) mit der angepassten Voigt-Funktion (Gl. (4.1), rot). Die mittlere Frequenz $f_{0}=60.0(1) \mathrm{GHz}$ aus der Anpassung ist als schwarze Linie eingezeichnet. (c) Auftragung der, wie in (a) und (b) ermittelten, Eigenfrequenzen von vier unterschiedlich dicken Wolfram-Schichten. In Rot ist die Anpassung der Funktion (4.2) dargestellt. Die aus der Anpassung bestimmte mittlere Schallgeschwindigkeit für Wolfram beträgt $v_{\mathrm{W}}=5103(108) \mathrm{m} \mathrm{s}^{-1}$.

unsere Wolfram-Filme $v_{\mathrm{W}}=5103(108) \mathrm{m} \mathrm{s}^{-1}$. Ein Wert der vergleichbar mit der bekannten Schallgeschwindigkeit von Wolfram $v_{\mathrm{W}, \mathrm{Lit}}=5220 \mathrm{~m} \mathrm{~s}^{-1}$ [73] ist.

\subsubsection{PPR Ergebnisse}

Wie bereits in einer früheren Veröffentlichung gezeigt [63], können mit Hilfe eines Ultrakurzzeitlaserpulses dynamische Schwingungen in einer Metall-IsolatorMultilage angeregt werden. In einer periodischen Struktur entspricht das Spektrum der Dynamik an der Oberfläche in etwa den Frequenzen in der Bandlücke nach Rytov (siehe Gl. (2.10)). Um die spektralen Eigenschaften der Strukturierung von dem Ort der Anregung zu trennen und die Dynamik genauer zu erforschen, habe ich in dieser Arbeit $\mathrm{MgO} / \mathrm{ZrO}_{2}$ Multilagen untersucht. Diese sind für die $800 \mathrm{~nm}$ Wellenlänge des Lasers transparent und absorbieren dementsprechend nur wenig Energie aus dem Laser. Als Ort der Laserabsorption und damit der Anregung der Dynamik, dient eine Wolframschicht auf der Multilage. Diese wurde 
mit Hilfe einer Blende in der PLD mit einer lateral variablen Dicke aufgewachsen. Mit dem PPR Aufbau (siehe Kapitel 3.3) wurden Dynamikmessungen auf der Wolframschicht von zwei Multilagen durchgeführt. Die eine mit der Struktur $\mathrm{W}+\left[(\mathrm{MgO})_{10 \mathrm{~nm}} /\left(\mathrm{ZrO}_{2}\right)_{10 \mathrm{~nm}}\right]_{50}$ und die andere mit halber Doppellagendicke $\mathrm{W}+\left[(\mathrm{MgO})_{5 \mathrm{~nm}} /\left(\mathrm{ZrO}_{2}\right)_{5 \mathrm{~nm}}\right]_{50}$.

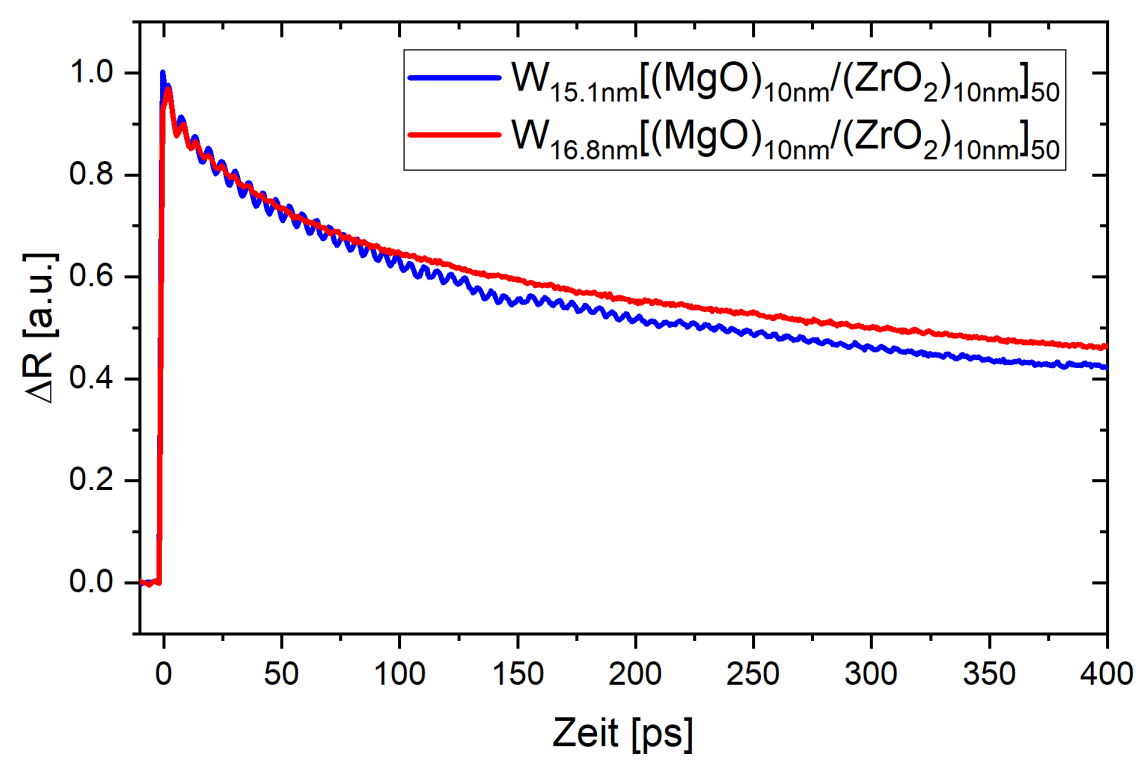

Abbildung 4.2: Änderung der PPR-Reflexion an einer Multilagenprobe aus $\left[(\mathrm{MgO})_{10 \mathrm{~nm}} /\left(\mathrm{ZrO}_{2}\right)_{10 \mathrm{~nm}}\right]_{50}$. Die rote Kurve wurde an einer Stelle mit $16.8 \mathrm{~nm}$ und die blaue Kurve an einer Stelle mit $15.1 \mathrm{~nm}$ Schichtdicke des Wolframs gemessen.

Zwei Beispielmessungen sind in Abb. 4.2 zu sehen. Um an die elastische Dynamik an der Probenoberfläche zu gelangen, wird nun der thermische Hintergrund (also Änderung der Reflektivität durch Erhöhung der Temperatur durch den PumpPuls, siehe Kapitel 3.4) mit einem doppelt exponentiellen Abfall von den Messdaten abgezogen. In Abb. 4.3 ist die so extrahierte Probenoberflächendynamik für die 10/10 Probe (a) und 5/5 Probe (b) als Wasserfalldiagramm für Messungen an verschiedenen Probenpositionen dargestellt. Die über PLD-Aufdampfrate und 
Geschwindigkeit der Blende bestimmten sowie mittels Röntgenbeugung geprüften Schichtdicken sind über die Umrechnungsfunktionen

$$
\begin{aligned}
10 / 10: & d(x)=-0.29(10) \mathrm{nm} \mathrm{mm}^{-1} \cdot x+19.4(2) \mathrm{nm} \\
5 / 5: & d(x)=0.45(10) \mathrm{nm} \mathrm{mm}^{-1} \cdot x+4.5(2) \mathrm{nm}
\end{aligned}
$$

im Experiment zurückverfolgbar.

(a)

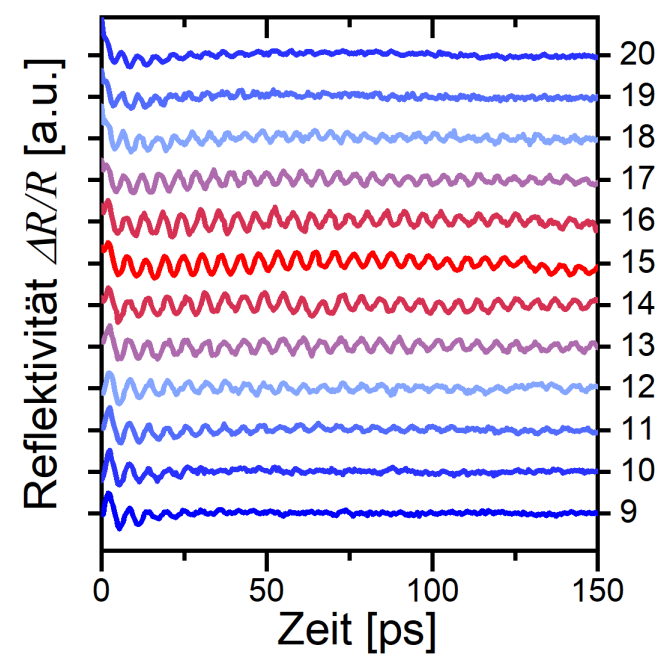

(b)

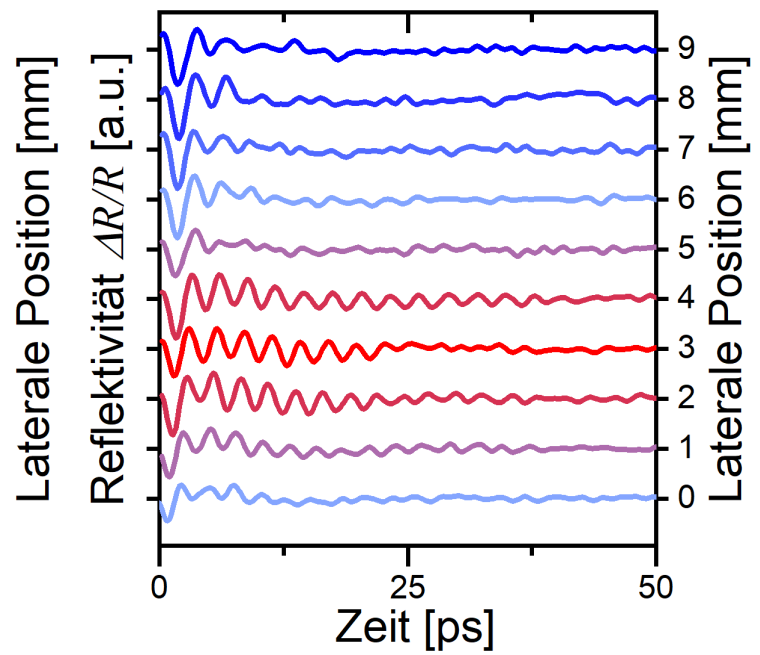

Abbildung 4.3: Änderung der PPR-Reflektivität nach Abzug des thermischen Hintergrunds (vgl. Kap. 3.3) für ein Übergitter aus (a) $\mathrm{W}+\left[(\mathrm{MgO})_{10 \mathrm{~nm}} /\left(\mathrm{ZrO}_{2}\right)_{10 \mathrm{~nm}}\right]_{50}$ und (b) $\mathrm{W}+\left[(\mathrm{MgO})_{5 \mathrm{~nm}} /\left(\mathrm{ZrO}_{2}\right)_{5 \mathrm{~nm}}\right]_{50}$ an Positionen mit verschiedenen Schichtdicken des Wolframfilms (jeweils rechte Achse).

An den Messungen ist gut zu erkennen, dass wir bei jeder Messung direkt nach $t=0$ eine Oszillation an der Probenoberfläche beobachten können. An den Randpositionen der Proben sind diese Oszillationen stark gedämpft und schwingen innerhalb weniger Pikosekunden aus (blauer Bereich in Abb. 4.3). Um eine bestimmte Position herum jedoch beobachten wir, dass die Lebenszeit der Oszillation deutlich zunimmt und bei der 10/10-Probe auf über 150 ps wie auch bei der 5/5-Probe auf immerhin etwa 45 ps anwächst (rot eingefärbt).

Zum Bestimmen des Frequenzspektrums der Schwingungen berechnen wir zunächst die Autokorrelation der Messung und bilden anschließend das Spektrum über eine Fouriertransformation. Die Spektren der Messungen aus Abb. 4.2 sind ex- 
emplarisch in der Abb. 4.4 dargestellt. Für die Position mit $d_{\mathrm{W}}(15 \mathrm{~mm})=15.1 \mathrm{~nm}$

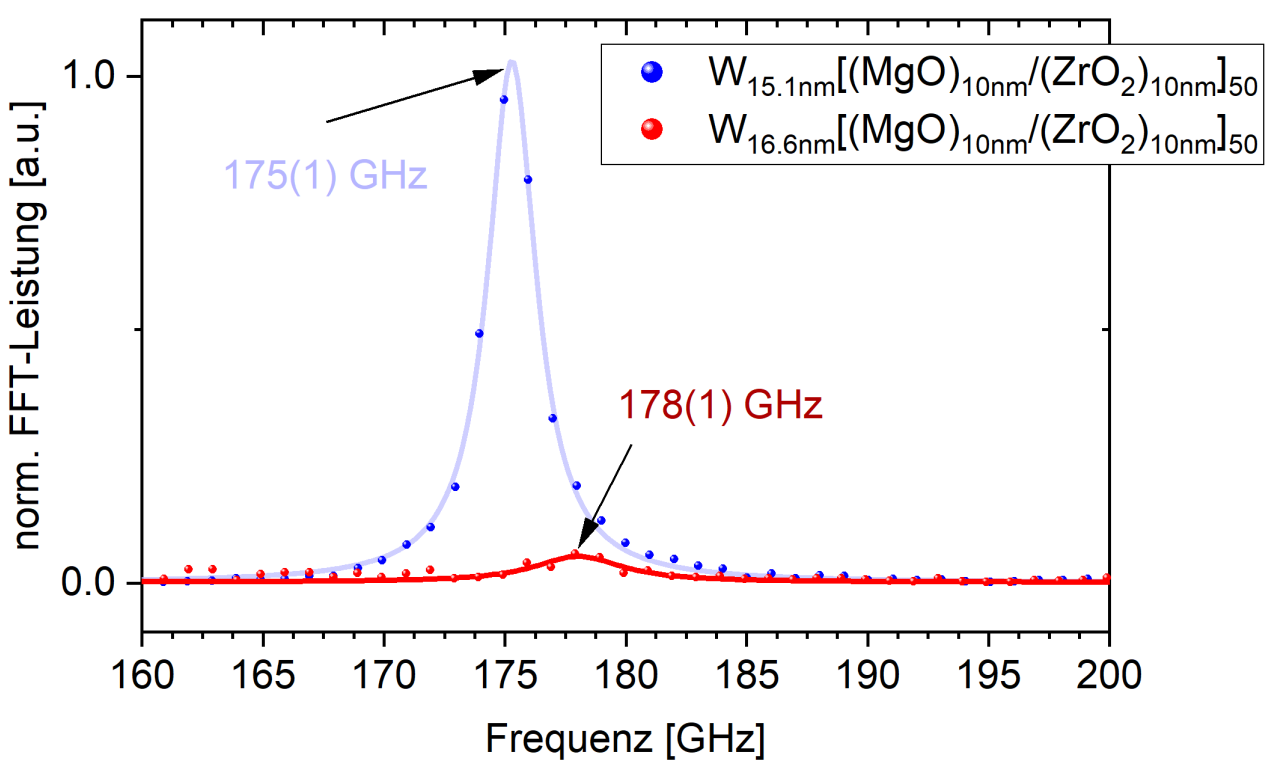

Abbildung 4.4: FFT-Spektrum der Messungen aus Abb. 4.2 (10/10 Probe). Die blauen Punkte sind an einer Stelle mit etwa $15.1 \mathrm{~nm}$ und die roten Punkte an einer Stelle mit $16.6 \mathrm{~nm}$ Dicke der Wolfram-Deckschicht gemessen worden. Vor dem Berechnen des FFT-Spektrums (hellblau und dunkelrot) wurde der thermische Hintergrund der Messung subtrahiert. Die eingezeichneten Mittelpunkte sind über das Anpassen einer Lorentzkurve bestimmt.

sehen wir eine deutliche Oszillation mit etwa $f=175(1) \mathrm{GHz}$. Für die Wolframdicke von $d_{\mathrm{W}}(10 \mathrm{~mm})=16.6 \mathrm{~nm}$ fällt die FFT-Amplitude aufgrund der Kurzlebigkeit der Oszillation im Messsignal im Vergleich sehr klein aus. Über das Anpassen einer Lorentzfunktion finden wir als zentrale Frequenz etwa $f=178(1)$ GHz. Die Spektren für beide Multilagen sind als Funktion der Wolframschichtdicke in Abb. 4.5 dargestellt. An den Spektren stellen wir zunächst fest, dass nicht nur die Stärke der Oszillation (Fourier-Leistung), sondern auch die Frequenz der beobachteten Oszillation stark von der Schichtdicke des Wolframs abhängt. Dies sehen wir besonders gut an Abb. 4.5 (a), aber auch in (b) ist derselbe Trend zu erkennen. Um dies noch ein wenig genauer zu untersuchen, sind die Mittenfrequenzen und die Spitzenfourierleistung der angepassten Lorentzkurven an alle Messungen in Abb. 4.6 aufgetragen. Die aus diesen Daten abgelesenen minimalen und maximalen Fre- 
(a)

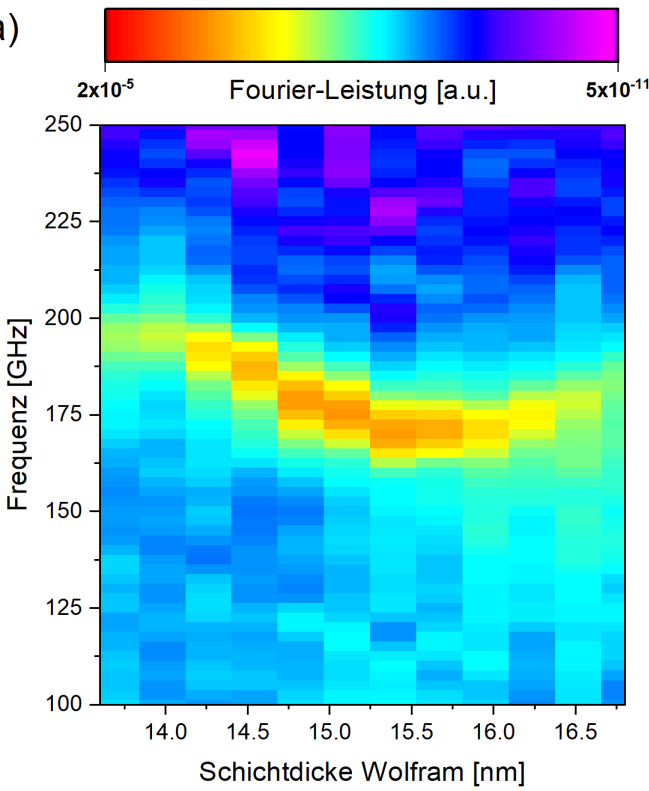

(b)
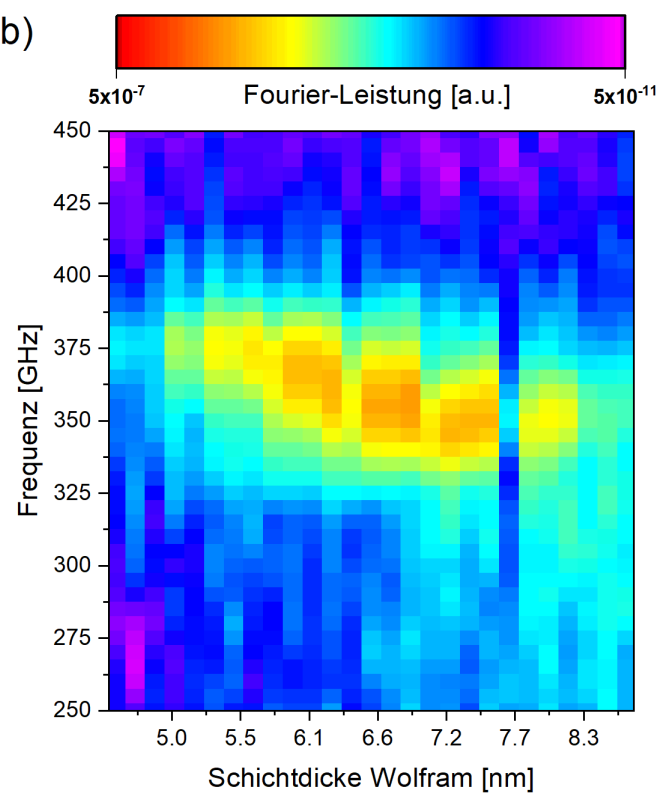

Abbildung 4.5: FFT-Spektren aller PPR-Messungen als Funktion der Wolframschichtdicke für (a) $\mathrm{W}+\left[(\mathrm{MgO})_{10 \mathrm{~nm}} /\left(\mathrm{ZrO}_{2}\right)_{10 \mathrm{~nm}}\right]_{50}$ und (b) $\mathrm{W}+\left[(\mathrm{MgO})_{5 \mathrm{~nm}} /\left(\mathrm{ZrO}_{2}\right)_{5 \mathrm{~nm}}\right]_{50}$. Die Farbskala ist logarithmisch.

quenzen, Resonanzfrequenz $f_{\mathrm{R}}$ und Wolframschichtdicke $\mathrm{d}_{\mathrm{R}}$ am Ort der stärksten Resonanz sind in Tabelle 4.1 aufgelistet. Ergänzend sind Werte aus Berechnungen der Bänder nach Rytov (Gleichung (2.10)) eingetragen. Dabei sind $f_{\text {Min. }}$ und $f_{\text {Max. }}$ jeweils die Frequenzen am Rand der ersten Bandlücke. $\Delta f$ ist die Differenz dieser Frequenzen, $f_{\mathrm{R}}$ die Frequenz in der Mitte der Lücke und $d_{\mathrm{R}}=\frac{v_{\mathrm{W}}}{2 f_{\mathrm{R}}}$ die Resonanzdicke im Wolfram für eine Schallwelle mit Geschwindigkeit $v_{\mathrm{W}}=5220 \mathrm{~m} \mathrm{~s}^{-1}$.

Wir sehen, dass die beobachteten Frequenzen in den Messungen denen entsprechen, die wir mit der theoretischen Berechnung der Rytov-Gleichung und den Literaturwerten für Dichte und Schallgeschwindigkeit für unsere $\mathrm{MgO}$ und $\mathrm{ZrO}_{2}$ Multilagen erhalten.

Wir haben dadurch nicht nur die Vorhersage der Rytov-Gleichung für die erste Bandlücke unserer Multilagen bestätigt (vgl. Kapitel 2.1), wir sehen auch, dass wir die Bandlücken der Multilagen als Funktion der Wolfram-Schichtdicke ausmessen können. Ist die Resonanzfrequenz der Wolframschicht auf die Bandlückenfrequenz angepasst, erhalten wir nach Anregung eine starke und vergleichsweise schwach 
(a)

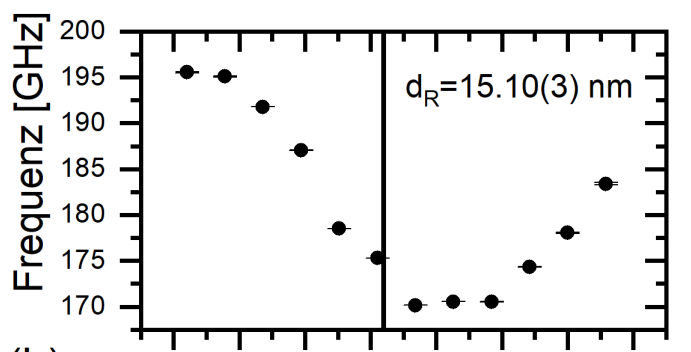

(b) $\quad 13.514 .014 .515 .015 .516 .016 .517 .0$

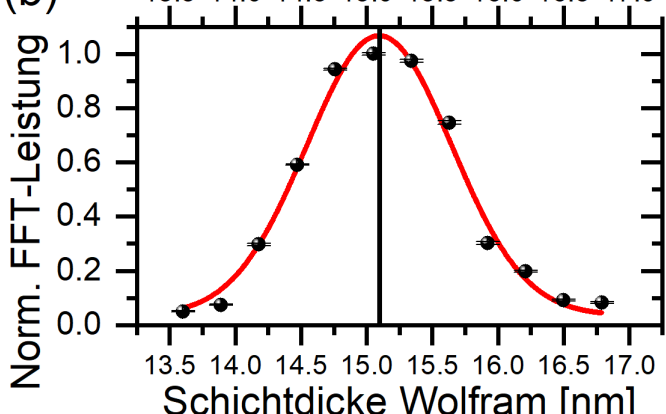

(c)

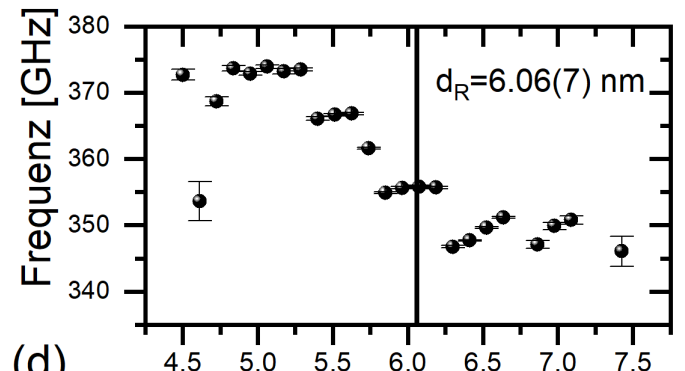

(d)

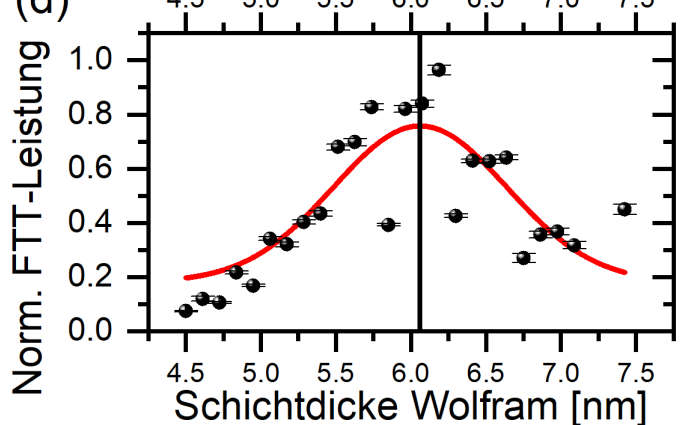

Abbildung 4.6: Parameter aus den Spektren durch das Anpassen einer Lorentzfunktion an die jeweilige FFT des Messungen. (a) Frequenz der dominanten Oszillation von $\mathrm{W}+\left[(\mathrm{MgO})_{10 \mathrm{~nm}} /\left(\mathrm{ZrO}_{2}\right)_{10 \mathrm{~nm}}\right]_{50}$ als Funktion der Schichtdicke und (b) die normierte Spitzen-Fourier-Leistung. (c) und (d) analog für die Probe $\mathrm{W}+\left[(\mathrm{MgO})_{5 \mathrm{~nm}} /\left(\mathrm{ZrO}_{2}\right)_{5 \mathrm{~nm}}\right]_{50}$. Die Resonanzschichtdicke $\mathrm{d}_{\mathrm{R}}$ wurde jeweils über den Mittelpunkt einer an die Fourierleistung angepassten Gaussfunktion (rot) bestimmt.

gedämpfte Schwingungsmode im Wolframfilm. Dies legt die Idee nahe, eine Multilage mit angepasster Deckschicht als optisch gepumpten, elastischen Resonator zu verwenden, z.B. um mit Hilfe magnetoelastischer Kopplung die akustische Resonanz zur Erzeugung einer magnetischen Oszillation zu nutzen. Simulationen zu dieser Idee werde ich im Kapitel 5.2 zeigen.

\subsubsection{Finite-Differenzen-Simulation}

Mit Hilfe der Pump-Probe-Spektroskopie habe ich im letzten Kapitel gezeigt, wie die akustische Bandlücke einer Multilage als Funktion der Deckschichtdicke untersucht werden kann. Am Punkt der Frequenzüberschneidung zwischen Bandlücke der Multilage und Resonanzfrequenz der Deckschicht beobachten wir eine nur schwach gedämpfte stehende Welle in der obersten Schicht. Wie bereits in 
Tabelle 4.1: Eigenschaften der Oszillation in den PPR Messungen der Multilagen $\mathrm{W}+\left[(\mathrm{MgO})_{10 \mathrm{~nm}} /\left(\mathrm{ZrO}_{2}\right)_{10 \mathrm{~nm}}\right]_{50}$ und $\mathrm{W}+\left[(\mathrm{MgO})_{5 \mathrm{~nm}} /\left(\mathrm{ZrO}_{2}\right)_{5 \mathrm{~nm}}\right]_{50}$. Bei den Werten in den Rytov-Spalten handelt es sich um die erste Bandlücke aus den Berechnungen wie in Abb. 2.4 für jeweils 10/10 und 5/5 Übergitter.

\begin{tabular}{|l|l|l|l|l|l|}
\hline Probe & $f_{\text {Min. }}$ & $f_{\text {Max. }}$ & $\Delta f$ & $f_{\mathrm{R}}$ & $\mathrm{d}_{R}$ \\
\hline $10 / 10$ & $170.2(1) \mathrm{GHz}$ & $195.5(7) \mathrm{GHz}$ & $25.3 \mathrm{GHz}$ & $175.3(1) \mathrm{GHz}$ & $15.1(3) \mathrm{nm}$ \\
\hline $5 / 5$ & $346.1(2) \mathrm{GHz}$ & $373.9(4) \mathrm{GHz}$ & $27.8 \mathrm{GHz}$ & $355.8(2) \mathrm{GHz}$ & $6.1(1) \mathrm{nm}$ \\
\hline Rytov $10 / 10$ & $170 \mathrm{GHz}$ & $192 \mathrm{GHz}$ & $22 \mathrm{GHz}$ & $181 \mathrm{GHz}$ & $14.4 \mathrm{~nm}$ \\
\hline Rytov $5 / 5$ & $340 \mathrm{GHz}$ & $384 \mathrm{GHz}$ & $44 \mathrm{GHz}$ & $362 \mathrm{GHz}$ & $7.2 \mathrm{~nm}$ \\
\hline
\end{tabular}

Kapitel 4.1.1 erwähnt, dient die Wolframschicht sowohl als Ort einer definierten Anregung der Probendynamik, als auch als Sonde für die Oberflächendynamik der Probe. Dabei blockiert diese Schicht jedoch den optischen Zugang ins Innere der Probe. Ohne die Deckschicht ist es zwar möglich ein Signal von der Dynamik aus dem Inneren der Probe zu bekommen [63], doch kann ohne Oberflächenmode die Bandlücke nicht ausgemessen werden. Um dennoch eine Idee von der Dynamik im Inneren der Probe mit Wolframschicht zu bekommen und damit auch, auf welchen Zeitskalen sich die Resonanz ausbildet, nutzen wir eine in unserer Arbeitsgruppe entwickelte Finite-Differenzen-Simulation. Details zu dieser Simulation sind in unseren Veröffentlichungen von 2016 [63] und 2017 [26] zu finden.

Mit Hilfe der Simulation habe ich die akustische Dynamik einer Multilage mit der Zusammensetzung $\left[(\mathrm{MgO})_{10 \mathrm{~nm}} /\left(\mathrm{ZrO}_{2}\right)_{10 \mathrm{~nm}}\right]_{50}$ und unterschiedlich dicken Wolframschichten berechnet. Für die nötige Diskretisierung wird das System in $0.25 \mathrm{~nm}$ große Zellen unterteilt und die Berechnung erfolgt in $0.1 \mathrm{fs}$ Zeitschritten. Der Zustand der simulierten Probe wird jede 1 ps auf die Festplatte gespeichert. Die genutzten Materialparameter sind im Anhang in Tabelle A.1 aufgeführt. In Abb. 4.7 (a) ist das simulierte Spektrum einer Multilage mit $14 \mathrm{~nm}$ Wolfram als Deckschicht dargestellt. Gut zu erkennen ist die Mode bei $f=182 \mathrm{GHz}$. Ihre Amplitude ist als Funktion der Position innerhalb der Probe mit dem Übergang zwischen Wolfram und Multilage bei $z=0$ dargestellt. Das Modenprofil bei der Resonanzfrequenz ist in Abb. 4.8 in blau dargestellt. In dem logarithmischen Plot gut zu erkennen ist die Schwingung in der Wolframschicht und die exponentiell abklingende Welle in die Multilage hinein. Auch sehen wir, dass die Schwingungsknoten einen Abstand von 


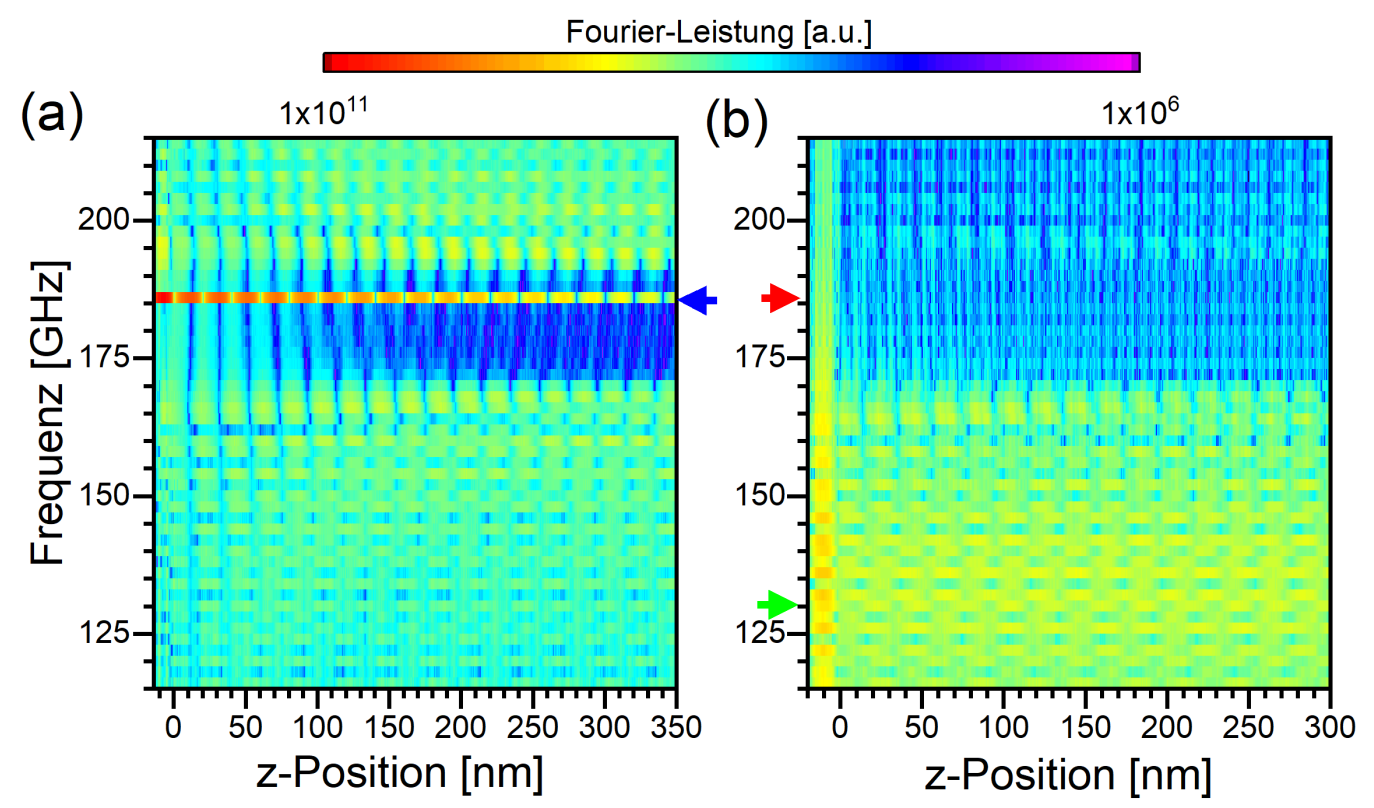

Abbildung 4.7: (a) Ausschnitt des FFT-Spektrums der elastischen Dynamik (elastische Verspannung $\sigma$ ) einer Multilagenprobe mit $14 \mathrm{~nm}$ Wolframschicht als Funktion der Position innerhalb der Probe (senkrecht zur Stapelrichtung). Gut zu erkennen ist die stehende Welle bei etwa $f=182 \mathrm{GHz}$. (b) Ausschnitt des FFTSpektrums für eine Multilage mit $20 \mathrm{~nm}$ Wolframschicht. Es sind im Vergleich mit (a) keine eindeutigen Moden zu erkennen. $z=0$ ist in allen Abbildungen der Übergang zwischen Wolframschicht und Multilage. Die farbigen Pfeile in (a) und (b) markieren die Moden aus Abb. 4.8 mit der selben Farbe.

$20 \mathrm{~nm}$ haben, folglich also die Doppellagen gegeneinander schwingen. Dies ist gerade die erste Bandlücke, wie wir sie in Kapitel 2.1 mit Hilfe der Rytov-Gleichung hergeleitet haben. Die Resonanzfrequenz liegt im Rahmen der FFT-Aufösung von $2 \mathrm{GHz}$ genau auf der Resonanzfrequenz, wie wir sie durch Einsetzen derselben Parameter in die analytische Lösung (2.10) erhalten $\left(f_{\mathrm{R}}=181 \mathrm{GHz}\right.$, siehe Tabelle 4.1).

In Abb. 4.7 (b) ist ein Spektrum wie in (a) abgebildet, jedoch mit Wolframschicht von $20 \mathrm{~nm}$ Dicke. Die theoretische Resonanzfrequenz dieser Wolframschicht liegt bei etwa $f_{\mathrm{R}}=\frac{v_{\mathrm{W}}}{2 \mathrm{~d}_{\mathrm{w}}}=130 \mathrm{GHz}$. Tatsächlich können wir in diesem Frequenzbereich eine spektral unscharfe und amplitudenschwache Oszillation im Wolfram sehen, die in die Probe hinein fast nicht gedämpft zu sein scheint. Bei der ursprünglichen Resonanzfrequenz von $181 \mathrm{GHz}$ ist weder eine ausgezeichnete Oszillation in der 


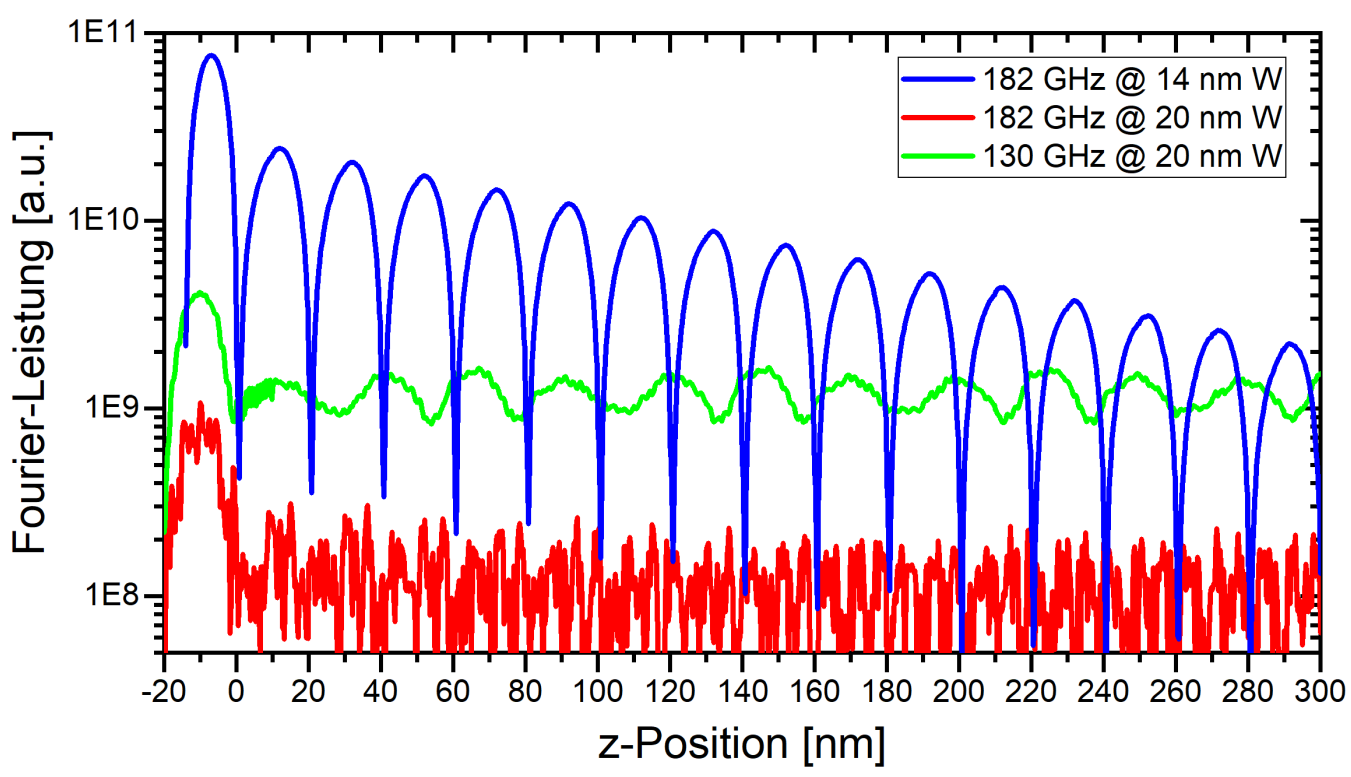

Abbildung 4.8: Ein Schnitt der Fourier-Leistung durch die Probe bei $f=182 \mathrm{GHz}$ für die Probe mit $14 \mathrm{~nm}$ Wolfram (blau) und je ein Schnitt bei $f=182 \mathrm{GHz}$ (rot) und $f=130 \mathrm{GHz}$ (grün) für die Multilage mit $20 \mathrm{~nm}$ Wolfram.

Wolframschicht noch eine Mode im Inneren der Probe zu erkennen. In Abb. 4.8 ebenfalls abgebildet ist ein Schnitt durch das Spektrum der $20 \mathrm{~nm}$ Probe sowohl bei $130 \mathrm{GHz}$ (grün) als auch bei $182 \mathrm{GHz}$ (rot). Wir stellen hier fest, dass bei der Frequenz von $182 \mathrm{GHz}$ keine sichtbare Mode in der Probe im Rahmen der Simulationsaufösung existiert. Einzig eine sehr schwache Eigenschwingung (vgl. Gl. (4.2)) im Wolfram ist zu beobachten. Diese ist zwei Größenordnungen kleiner als im Resonanzfall bei $d_{\mathrm{W}}=14 \mathrm{~nm}$. Bei $130 \mathrm{GHz}$ wiederum erkennen wir eine, dieses mal fast ungedämpfte, Oszillation vom Wolfram bis zum Substrat bei $z=$ $1 \mu \mathrm{m}$ (zwecks Darstellung nicht abgebildet).

Werfen wir nun einen Blick auf die zeitliche Entwicklung der Probendynamik: In Abb. 4.9 ist die elastische Verspannung als Funktion der z-Position in der Probe für verschiedene Zeitpunkte nach Anregung der $14 \mathrm{~nm}$ Wolframschicht dargestellt. In den einzelnen Zeitschritten ist gut zu erkennen, wie der Anregungspuls sich durch die Probe bewegt und dabei an jeder Grenzfläche an Amplitude verliert. Die reflektierten Wellen können konstruktiv interferieren und erzeugen bereits nach wenigen Pikosekunden das bereits bekannte Modenprofil der Oberflächenmode. 
Zum direkten Vergleich ist in Abb. 4.10 dieselbe Auftragung für die Multilage mit $20 \mathrm{~nm}$ Wolfram dargestellt. Auch hier sehen wir wieder den Anregungspuls sich durch die Probe bewegen, doch in diesem Fall kann sich in der Wolframschicht keine stehende Welle ausbilden. Die an den Grenzschichten reflektierten Wellen können in der Wolframschicht nicht mehr konstruktiv interferieren. Die Energie der Anregung fließst bereits zum größten Teil mit dem ersten Puls in das Substrat $\mathrm{ab}$. 


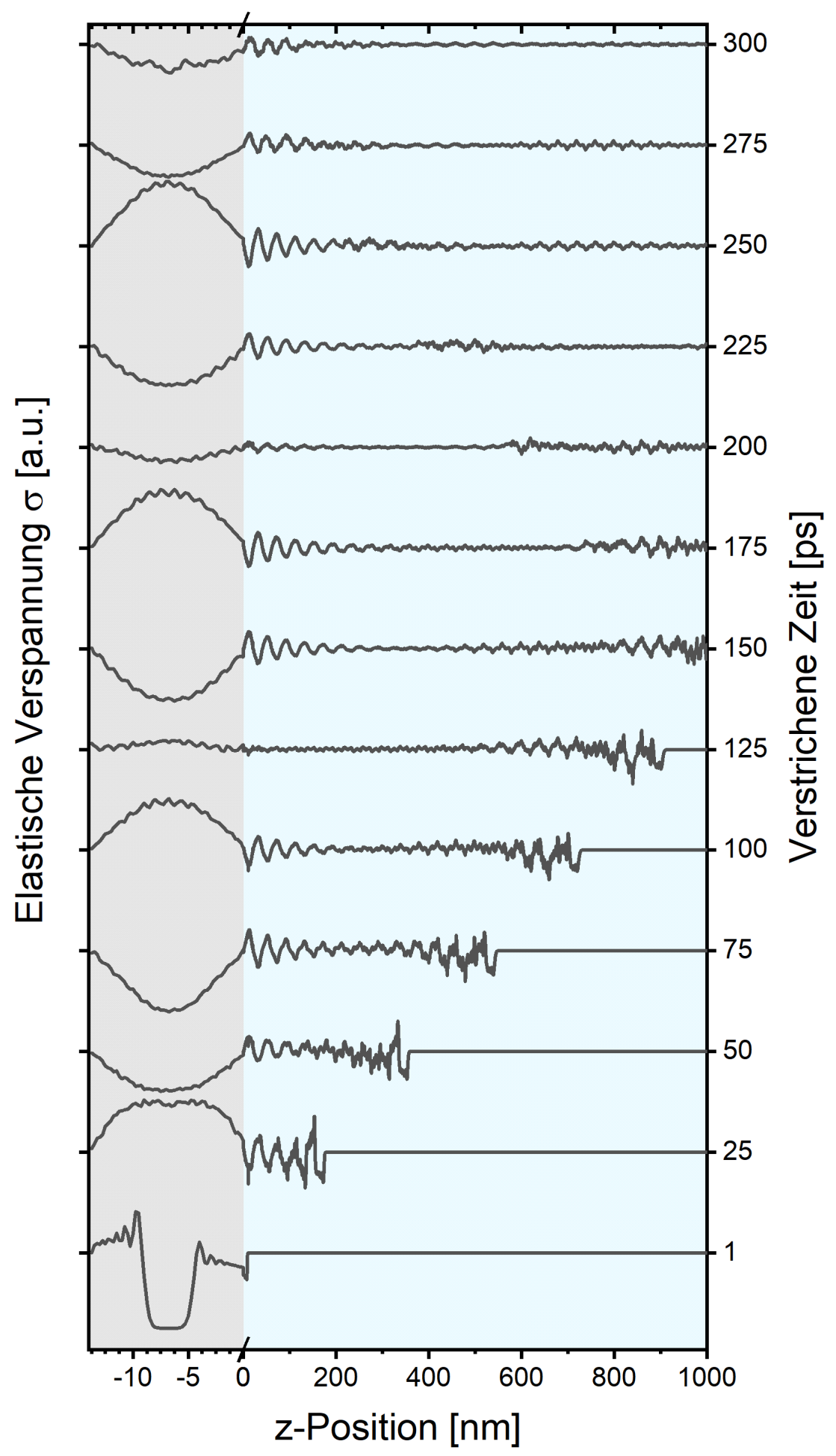

Abbildung 4.9: Elastischer Stress aus FDTD-Simulation für eine $\mathrm{W}_{14 \mathrm{~nm}}+\left[(\mathrm{MgO})_{10 \mathrm{~nm}} /\left(\mathrm{ZrO}_{2}\right)_{10 \mathrm{~nm}}\right]_{50}$ Multilagenprobe $\mathrm{zu}$ verschiedenen Zeitpunkten der Simulation. Die x-Achse ist für die ersten $14 \mathrm{~nm}$ (grau) gestreckt, um die Oberflächenmode im Wolfram hervorzuheben. 


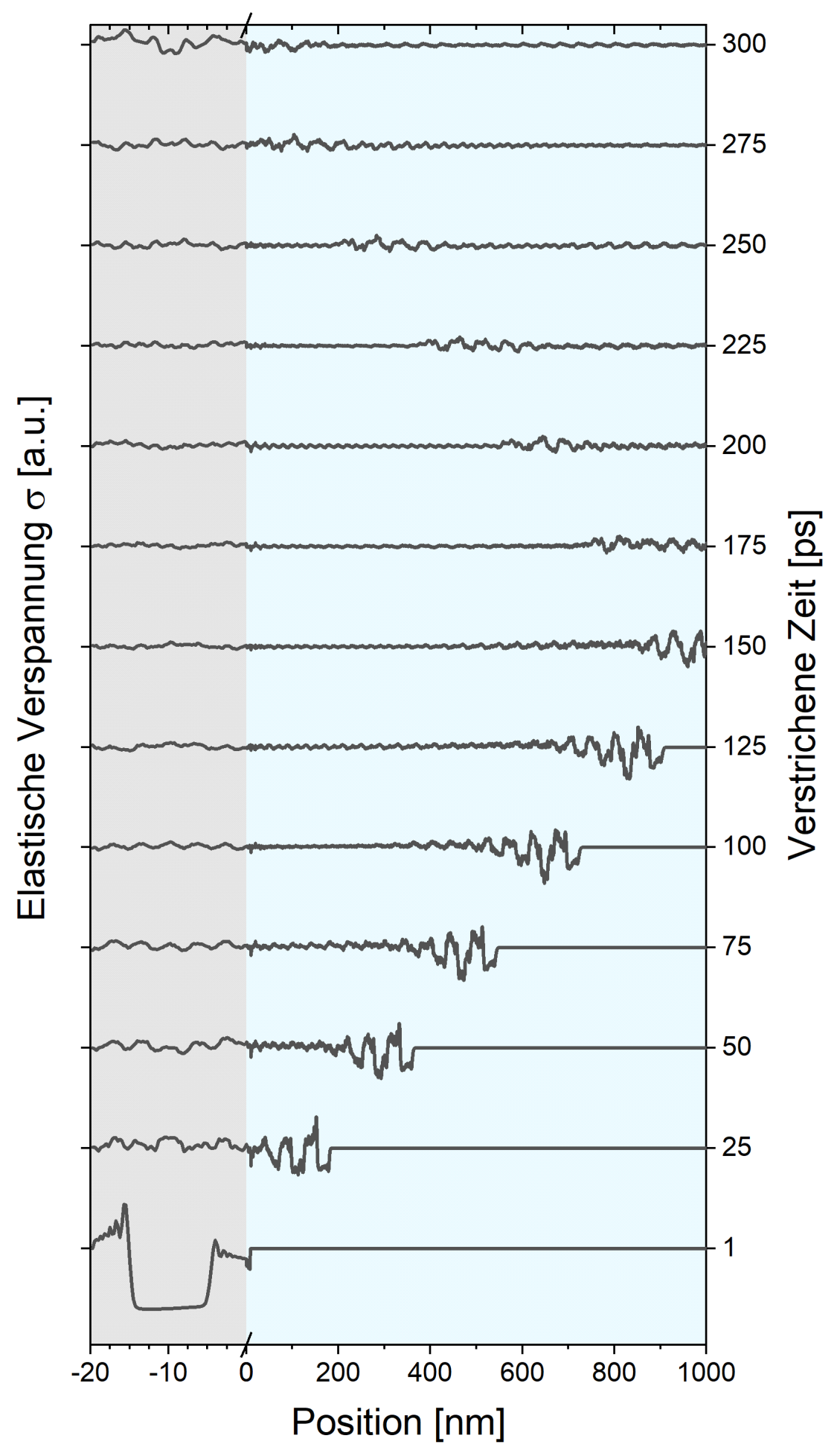

Abbildung 4.10: Elastischer Stress aus FDTD-Simulation für eine $\mathrm{W}_{20 \mathrm{~nm}}+\left[(\mathrm{MgO})_{10 \mathrm{~nm}} /\left(\mathrm{ZrO}_{2}\right)_{10 \mathrm{~nm}}\right]_{50}$ Multilagenprobe $\mathrm{zu}$ verschiedenen Zeitpunkten der Simulation. Die x-Achse ist für die ersten $20 \mathrm{~nm}$ (grau) gestreckt, um das Fehlen der Oberflächenmode im Wolfram hervorzuheben. 


\subsection{Wärmetransport in LMO/SMO}

An den Untersuchungen an $\mathrm{MgO} / \mathrm{ZrO}_{2}$ Multilagen konnten wir über die Resonanz in der Deckschicht aus Wolfram die akustische Bandlücke im oberen GHz Bereich beobachten. Im Fall der Übereinstimmung von Resonanzfrequenz der Wolframschicht und Bandlücke der Multilage, war eine nur schwach gedämpfte Oberflächenmode zu beobachten. Die Multilage hat als eine Art Bragg-Spiegel für die elastische Anregung fungiert. Ein Teil der Energie des Anregungspulses wurde an ihr reflektiert und so an der Probenoberfläche gehalten.

Wie bereits in den Kapitel 2.2.2 erwähnt, sind kohärente Gitterschwingungen, die Phononen, der Hauptträger des Wärmetransports in dielektrischen Materialien. Wir haben uns daher nach den Messungen an $\mathrm{MgO} / \mathrm{ZrO}_{2}$ gefragt, ob wir durch Verkleinern der Schichtdicke und damit verbunden das Hochskalieren der Frequenz in der Bandlücke (siehe Gl. (2.10)), mit Hilfe einer Multilage einen Teil der Wärmeenergie blockieren können. Für diese Untersuchung müssen wir die Multilagenschichtdicken so verkleinern, dass wir mit den Frequenzen in den typischen Bereich für zum thermischen Transport relevante Phononen von mindestens wenigen $\mathrm{THz}$ vorstoßen [65]. Dabei kommen wir in einen Bereich, wo wir nicht mehr nur von Multilagen, sondern von Übergittern sprechen (vgl. Kapitel 2.1). Bei den in diesem Abschnitt untersuchten Proben, handelt es sich um $\mathrm{LaMnO}_{3} / \mathrm{SrMnO}_{3}$-Übergitter, welche mit Hilfe der MAD-Methode hergestellt wurden (siehe Kapitel 3.1.3).

Das Untersuchen der Probendynamik mit dem PPR im THz-Bereich ist aufwendig: Zum einen ist die zeitliche Auflösung durch die Pulslänge limitiert, zum anderen auch die räumliche Auflösung des Experimentes durch die Eindringtiefe des Lasers. Ist die Eindringtiefe des Laserlichtes größer als die einzelnen Schichtdicken im Übergitter, oder genauer gesagt, die Wellenlängen der zu untersuchenden Phononen, so kann aus der Reflektivität nicht mehr auf die eigentliche Phononendynamik geschlossen werden.

Aus diesem Grund haben wir für die Untersuchung des Phononentransportes in den Übergittern statt der Reflektivitätsdynamik eine andere Messgröße gewählt: Für diese Messungen ziehen wir die Oberflächentemperatur der Probe heran. Über sie erhalten wir einen direkteren Zugang zum Wärmetransport der untersuchenten Übergitter. Die Messmethode ist im Detail im TTR-Kapitel 3.4.1 beschrieben. 


\subsubsection{TTR an $\mathrm{LMO}_{m} / \mathrm{SMO}_{n}$-Übergittern}

In diesem Kapitel stelle ich TTR-Messungen der Wärmeleitfähigkeit $\kappa$ von $(\mathrm{LMO})_{m} /(\mathrm{SMO})_{n}$ mit $m / n=1$ und $m / n=2$ vor. Die Auswertung der Messkurven erfolgte dabei wie bereits in Kapitel 3.4.1.4 dargestellt. Die Anzahl der Wiederholungen der $(\mathrm{LMO})_{m} /(\mathrm{SMO})_{n}$-Schichten wurde so gewählt, dass die Gesamtdicke des Übergitters bei allen Proben in einem Bereich von $20 \mathrm{~nm}$ bis $60 \mathrm{~nm}$ liegt. Eine Übersicht der Proben ist in Tabelle $3.1 \mathrm{zu}$ finden.

In Abb. 4.11 (a) sind die über jeweils drei unabhängige Messungen angepassten Wärmeleitfähigkeiten als Funktion der Übergitter-Periode $\Lambda$ dargestellt. Der eingezeichnete Fehler ist die Standardabweichung der Mittelung. Zur vereinfachten Darstellung wird im weiteren Verlauf die Übergitter-Periode auf die mittleren Gitterkonstante $c$ als $\Lambda / c$ normiert. $c$ wurde mit Hilfe von Röntgendiffraktometrie (XRD) zu $c=0.382 \mathrm{~nm}$ für $m / n=1$ und $c=0.384 \mathrm{~nm}$ bis $0.385 \mathrm{~nm}$ für $m / n=2$ bestimmt. In Blau dargestellt sind die Wärmeleitfähigkeiten von $m / n=1$. Bei den Quadraten und Dreiecken handelt es sich jeweils um verschiedene Probenserien, die jedoch mit der gleichen Übergitter-Periode und Gesamtschicktdicke hergestellt wurden.

Die Parameter für das TTR-Modell (Kapitel 3.4.1.2) sind im Anhang in Tabelle A.1 aufgeführt. Das Modell besteht aus einer Kupferschicht, einem Übergitter und dem Substrat (STO). Als freie Parameter des Modells passen wir $\kappa$ und die Grenzflächenleitfähigkeiten $h_{12}$ und $h_{23}$ an die Messdaten an. Für die Probenreihe $m / n=1$ und $m / n=2$ passen wir jeweils ein $h_{12}$ und $h_{23}$ für alle Proben der Reihe an. $\kappa$ bestimmen wir für jede Probe individuell. Die Messdaten sind in Tabelle 4.3 aufgelistet. Als Grenzflächenleitfähigkeiten haben wir für die Grenzfläche ÜG-Substrat $h_{23}=20(1) \mathrm{GW} \mathrm{m}^{-2} \mathrm{~K}^{-1}$, für $m / n=1$ zum Kupferfilm $h_{12}=0.087(1) \mathrm{GW} \mathrm{m}^{-2} \mathrm{~K}^{-1}$ und für $m / n=2 h_{12}=0.009(1) \mathrm{GW} \mathrm{m}^{-2} \mathrm{~K}^{-1}$ bestimmt.

Der Vergleichswert in Abb. $4.11 \kappa_{\mathrm{LMO}}=1.25(14) \mathrm{W} \mathrm{m}^{-1} \mathrm{~K}^{-1}$ wurde mit Hilfe des TTR an einer $30 \mathrm{~nm}$ dicken LMO Schicht gemessen. Für SMO ziehen wir den Literaturwert von $\kappa_{\mathrm{SMO}}=4.5 \mathrm{~W} \mathrm{~m}^{-1} \mathrm{~K}^{-1}$ [101] heran. Mit der Ausnahme des rot umrandeten Datenpunktes können wir sowohl bei $m / n=1$ als auch bei $m / n=2$ eine klare Abhängigkeit der Wärmeleitfähigkeit von der Übergitter-Periode $\Lambda=$ 
$(m+n) \cdot c$ erkennen. Mit kleiner Übergitter-Periode fällt die Wärmeleitfähigkeit der Übergitter. Dabei zeigen $m / n=2$ ÜG eine deutlich geringere Wärmeleitfähigkeit als $m / n=1 \ddot{\mathrm{UG}}$.

(a)

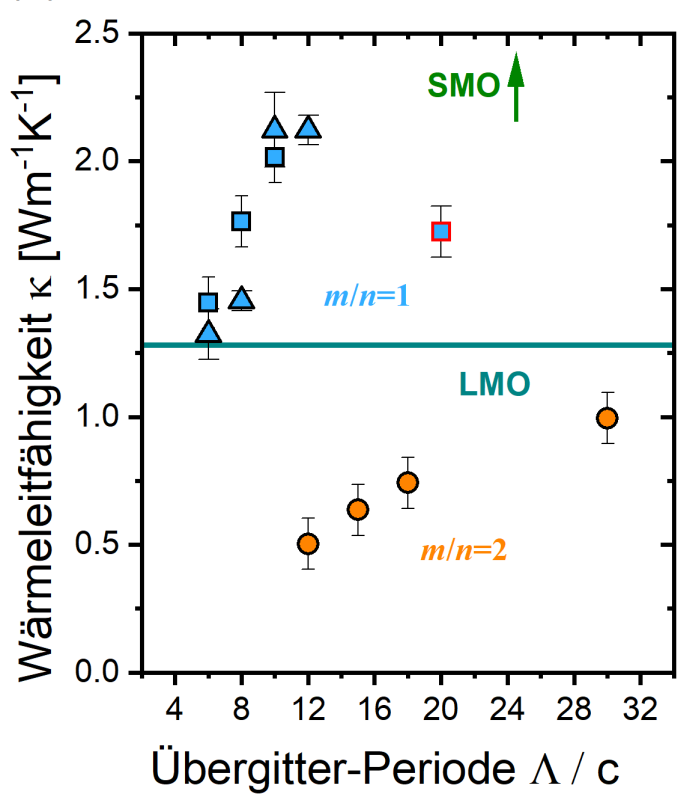

(b)

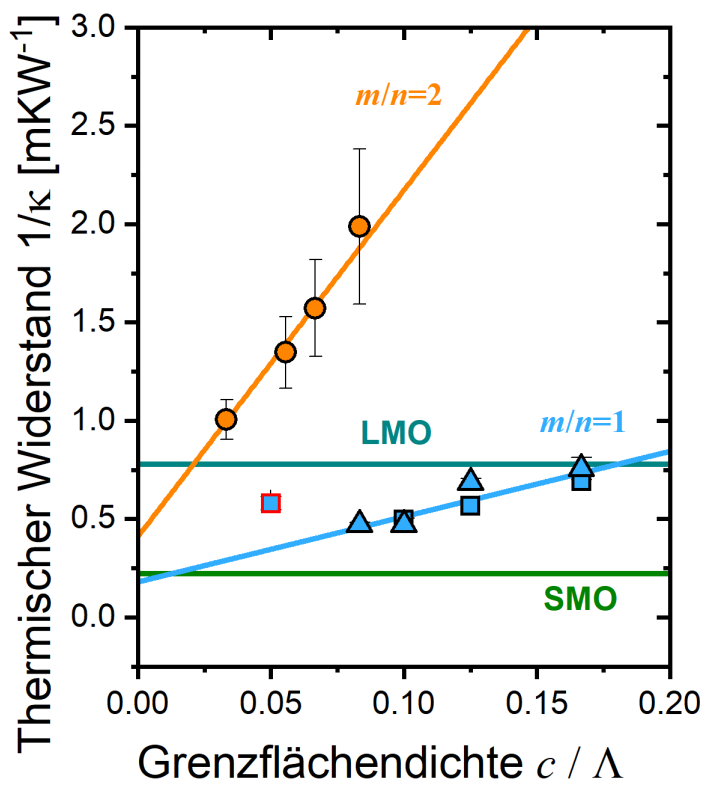

Abbildung 4.11: (a) TTR-Messungen der Wärmeleitfähigkeit $\kappa$ für $m / n=2$ LMO/SMO-Übergitter (orange) und $m / n=1$ ÜG (blau). (b) Thermischer Widerstand für LMO/SMO-Übergitter mit einem LMO/SMO-Verhältnis von 1:1 (blau) und 2:1 (orange) als Funktion der Grenzflächendichte $c / \Lambda$ und den Referenzwerten für LMO (mit TTR gemessen) und SMO [101]. Der lineare Fit für $m / n=1$ wurde an alle Punkte außer $c / \Lambda=0.05$ angepasst.

Als einfaches Modell der Wärmeleitfähigkeit der Übergitter können wir Schichten und Grenzflächen jeweils als einen thermischen Widerstand betrachten. Der Gesamtwiderstand des Übergitters ergibt sich dann als Reihenschaltung [32, 105]:

$$
\frac{1}{\kappa}=\frac{1}{\kappa_{\mathrm{V}}}+\frac{2}{h \Lambda}
$$

Hier ist $h$ die Grenzflächenleitfähigkeit (in $\mathrm{W} \mathrm{m}^{-2} \mathrm{~K}^{-1}$ ) zwischen den Schichten des Übergitters und

$$
\kappa_{\mathrm{V}}=\frac{(m+n)}{\left(m / \kappa_{\mathrm{m}}+n / \kappa_{\mathrm{n}}\right)}
$$


Tabelle 4.2: Resultate der angepassten Gleichung (4.4) an die TTR-Messdaten in Abb. 4.11.

\begin{tabular}{|l|l|l|}
\hline $\mathrm{m} / \mathrm{n}$ & $\kappa_{\mathrm{V}}$ & $h\left[\mathrm{GW} \mathrm{m}^{-2} \mathrm{~K}^{-1}\right]$ \\
\hline 1 & $5.6(26)$ & $1.6(3)$ \\
\hline 2 & $2.4(4)$ & $0.30(3)$ \\
\hline
\end{tabular}

die Wärmeleitfähigkeit eines Übergitterkristalls aus zwei unendlich ausgedehnten Schichten bzw. der gewichtete Mittelwert aus den beiden materialspezifischen Werten für $\kappa$. Tragen wir die Messdaten aus (a) als den thermischen Widerstand $1 / \kappa$ gegen die Grenzflächendichte $c / \lambda$ auf (Abb. $4.11(\mathrm{~b})$ ), sehen wir direkt den linearen Trend aus Gleichung (4.4). Für $m / n=1$ wurde die Gleichung an acht Messpunkte und für $m / n=2$ an vier Messpunkte angepasst. Der rot umrandete Punkt wurde von der linearen Anpassung ausgenommen. Eine Erklärung folgt zu einem späteren Zeitpunkt. Die Ergebnisse der angepassten Gleichung (4.4) sind in Tabelle 4.2 aufgelistet.

Wir erkennen, dass zumindest bei dicken Schichten die Wärmeleitfähigkeit des Gitters von $m / n=1$ bei etwa der doppelten Wärmeleitfähigkeit des $m / n=2$ Gitters liegt. Die Wärmeleitfähigkeit der Grenzflächen $h \mathrm{im} m / n=1$ ist etwa viermal so hoch.

Zur Erklärung dieser Unterschiede haben Dr. Vladimir Roddatis und Dr. Ulrich Roß die atomare Struktur solcher LMO/SMO-Übergitter mit Hilfe von Rastertransmissionselektronenmikroskopie (HAADF-STEM und (iDPC)-STEM [106]) untersucht. Dr. Igor Maznichenko und Dr. Sergey Ostanin, von der Universität Halle, haben DFT-Rechnungen zu diesen Strukturen durchgeführt. Das übereinstimmende Ergebnis [80] dieser Untersuchungen ist, dass der Mn-O-Mn Bindungswinkel von SMO in allen Übergittern etwa $\varphi_{\mathrm{SMO}}=180^{\circ}$ ist. Dies ist auch der charakteristische Wert für Bulk-SMO [107]. In den $m / n=2$ Schichten zeigt das LMO einen Mn-O-Mn Bindungswinkel von $\varphi_{\mathrm{LMO}}=165(3)^{\circ}$, was in etwa dem Wert von rhomboedrischen LMO entspricht [84, 108]. Im $m / n=1$ Übergitter hingegen zeigt das LMO einen Bindungswinkel von $\varphi_{\mathrm{LMO}}=180(3)^{\circ}$ identisch zum SMO. Für eine detaillierte Analyse dieser Ergebnisse verweise ich auf [80]. Eine weitere Veröffentlichung, welche die Ursachen für die Strukturunterschiede in $m / n=1,2$ 
LMO/SMO Übergittern detaillierter untersucht, befindet sich von Prof. Dr. Moshnyaga et al. noch in Vorbereitung.

An dieser Stelle sei nur das für den thermischen Transport relevante Ergebnis kurz festgehalten: Bei einem SMO/LMO Schichtdickenverhältnis von 2:1 wächst die SMO Schicht in einer kubischen $(\mathrm{Pm} \overline{3} \mathrm{~m})$ und die LMO-Schicht in einer rhomboedrischen $(\mathrm{r} \overline{3} \mathrm{c})$ Struktur auf. In den Übergittern mit einem Verhältnis von 1:1 wachsen sowohl SMO als auch LMO in einer kubischen Struktur (Pm̄̄m) auf. Die Grenzfläche zwischen den Schichten unterscheidet sich strukturell daher nicht so stark wie bei $m / n=2$. Das LMO in der Probe $\Lambda / c=20$ (rot umrandeter Punkt in Abb. 4.11) zeigt trotz $m / n=1$ einen Mn-O-Mn Bindungswinkel wie in den $m / n=2$ Proben. Dies legt nahe, dass in Übergittern mit dicken Lagen das LMO auch bei $m / n=1$ wieder in die rhomboedrische Struktur wechselt. Ein Zusammenhang zwischen niedriger Wärmeleitfähigkeit in LMO mit rhomboedrischer Struktur wurde bereits von Cohen et al. aufgestellt [109].

Wir können das Angleichen der Gitterstruktur auch direkt mit der Änderung der Grenzflächenleitfähigkeit $h$ in Verbindung bringen: Ein großer Unterschied in der Gitterstruktur an der Grenzfläche führt offensichtlich zu einer kleinen Grenzflächenleitfähigkeit, während gleiche Gitterstrukturen den thermischen Transport an der Grenzfläche weniger behindern. Erinnern wir uns an die berechnete Phononendispersion von LMO und SMO in Abbildung 2.14, so lässt sich auch vermuten, dass ein angepasstes Phononenspektrum zu einer großen Grenzflächenleitfähigkeit führt, während ein starker Unterschied zwischen den Bändern zu reduzierten Grenzflächenleitfähigkeiten führt. Was wir an der Berechnung des Phononenspektrums außerdem sehen, ist die Erhöhung der Grenzfrequenz der akustischen Phononen zwischen rhomboedrischen und kubischen LMO um fast das Dreifache auf etwa 6.6 THz (Vergleiche Dispersion in Abb. 2.14). Damit erhöht sich die mittlere Geschwindigkeit dieser Phononen, was sich nach Gleichung (2.24) direkt auf die Wärmeleitfähigkeit der LMO-Schicht auswirkt.

Um diese Überlegung zu stützen, berechne ich mit Hilfe unserer BK-Simulation (siehe Kapitel 2.2.3) die Phononenbandstruktur der verschiedenen Übergitter mit kubischem LMO für $m / n=1$ Übergitter und rhomboedrischem LMO für $m / n=2$ Übergitter. Weiterhin bestimme ich über die Gleichung (2.28) die Wärmeleitfähigkeit der Proben. Zwei solcher Bandstrukturen sind exemplarisch in Abbildung 4.13 
(a) und (b) entlang der ( $\left.\begin{array}{lll}0 & 0 & 1\end{array}\right)$-Kristallrichtung abgebildet. Für den Vergleich der beiden Gitter gehen wir zunächst von einer konstanten mittleren freien Weglänge aller beteiligter Phononen von 20 u.c. aus.

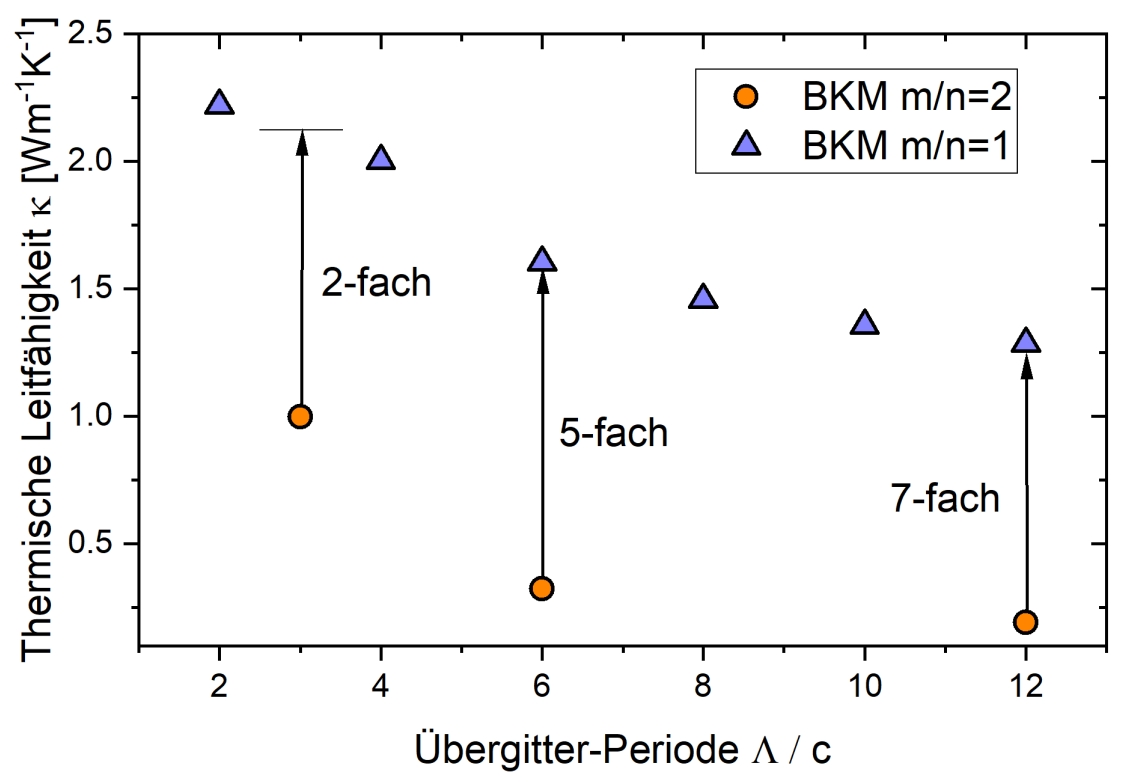

Abbildung 4.12: Thermische Leitfähigkeit für LMO/SMO-Übergitter mit einem LMO/SMO-Verhältnis von 1:1 (blau) und 2:1 (orange) als Funktion der ÜbergitterPeriode $\Lambda / c$ berechnet nach dem BK-Modell (Kapitel 2.2.3) mit konstanter freier Weglänge für alle Phononen von 20 u.c.. Für $m / n=1$ wurde kubisches LMO, für $m / n=2$ rhomboedrisches LMO angenommen. Zusätzlich eingezeichnet sind die relativen Unterschiede der Wärmeleitfähigkeiten bei ähnlicher Übergitter-Periode zwischen $m / n=1$ und 2 .

In Abbildung 4.12 ist am Vergleich der Modelle gut zu erkennen, dass unter Berücksichtigung der LMO-Gitterstruktur auch die Berechnungen der Bandstruktur und die daraus abgeleitete Wärmeleitfähigkeit ein ähnliches Verhältnis wie in den Messungen zeigt.

Bei der für die Simulation als konstant gewählten, mittleren freien Weglänge für alle Phononen von $\xi=20$ u.c., lässt sich aus den Berechnungen bis $\lambda / c=12$ noch kein Minimum der Wärmeleitfähigkeit wie bei CTO/STO [39] erkennen.

Wir nutzen daher nun die Phononenspektren und die Gleichung (2.30) um die mittlere freie Weglänge $\xi$ der am thermischen Transport beteiligten Phononen 
durch Anpassen an die Messdaten abzuschätzen. Diese sind für die $m / n=1$ und $m / n=2$ Messungen in Abbildung 4.13 (c) aufgetragen. Wir sehen, dass wir nur

(a)

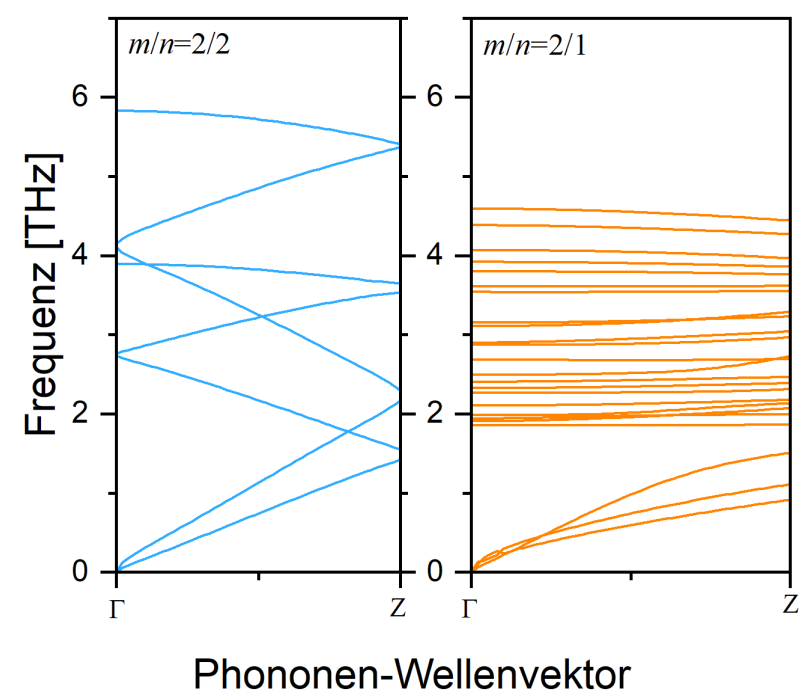

(c)

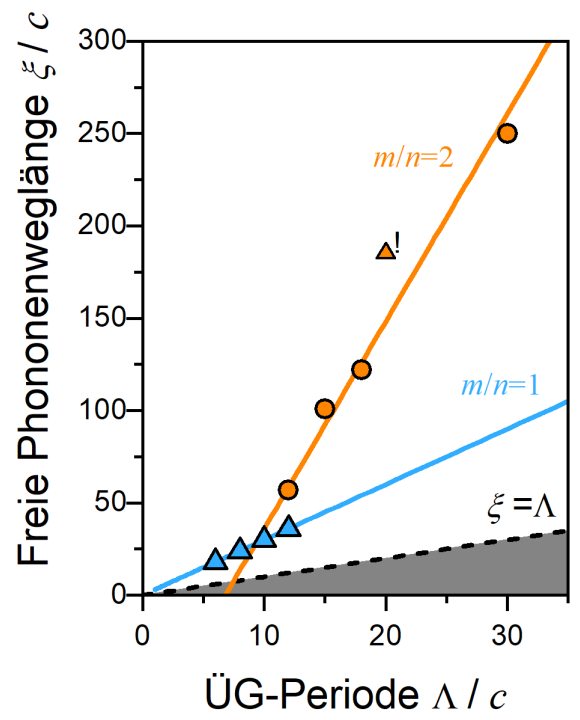

Abbildung 4.13: Phononenbandstrukturen entlang der $\mathrm{q}_{\mathrm{z}}$-Richtung für (a) $(\mathrm{LMO})_{2} /(\mathrm{SMO})_{2}$ und (b) $(\mathrm{LMO})_{2} /(\mathrm{SMO})_{1}$. (c) Durch Anpassen von Gleichung (2.30) an die TTR-Messdaten ermittelte mittlere freie Weglänge $\xi$ als Funktion der Übergitter-Periode $\Lambda / c$. Der Bereich $\xi<\Lambda$ ist schattiert. Das Dreieck in orange mit Ausrufezeichen ist ein Messpunkt des $m / n=1$ Übergitter.

bei $m / n=1$-Übergittern mit hoher Grenzfächenleitfähigkeit (vgl. Tabelle 4.2) einen phononischen Wärmetransport mit $\xi>\Lambda$ bei den dünnsten Übergitterperioden erwarten können (vgl. Abb. 2.10). Der Trend von $\xi$ bei $m / n=2$ legt nahe, dass bei diesen der Abstand der Grenzflächen die mittlere freie Weglänge bis zu den kleinsten Übergitterperioden dominiert und wir daher keinen kohärenten Phononentransport erwarten können.

Aus diesem Grund wählen wir für die weitere Untersuchung des Wärmetransports die $m / n=1$ Übergittern als unser Modellsystem. Weitere Messungen an diesem System folgen im nächsten Kapitel. 
Tabelle 4.3: TTR-Messdaten an $m / n=1$ und $m / n=2$ Übergittern. Für $m / n=1$ wurden zwei Probenserien mit strukturell identischen Proben vermessen. Diese Serien sind nach ihrer Herstellungszeit in der Tabelle in „Neu“ und „Alt" unterteilt.

\begin{tabular}{|c|c|c|c|c|c|c|}
\hline & & & & $\mathrm{eu}$ & & lt \\
\hline $\mathrm{m}: \mathrm{n}$ & $\Lambda / c$ & $c / \Lambda$ & $\kappa$ & $1 / \kappa$ & $\kappa$ & $1 / \kappa$ \\
\hline $3: 3$ & 6 & 0.17 & $1.3(1)$ & $0.76(6)$ & $1.4(1)$ & $0.73(6)$ \\
\hline $4: 4$ & 8 & 0.125 & $1.5(1)$ & $0.69(2)$ & $1.8(1)$ & $0.57(4)$ \\
\hline $5: 5$ & 10 & 0.1 & $2.1(2)$ & $0.47(4)$ & $2.0(1)$ & $0.50(3)$ \\
\hline $6: 6$ & 12 & 0.083 & $2.1(1)$ & $0.47(2)$ & - & - \\
\hline $10: 10$ & 20 & 0.05 & - & - & $1.7(1)$ & $0.58(4)$ \\
\hline $8: 4$ & 12 & 0.083 & - & - & $0.5(1)$ & $2.0(4)$ \\
\hline $10: 5$ & 15 & 0.067 & - & - & $0.6(1)$ & $1.6(3)$ \\
\hline $12: 6$ & 18 & 0.056 & - & - & $0.7(1)$ & $1.3(2)$ \\
\hline $20: 10$ & 30 & 0.033 & - & - & $1.0(1)$ & $1.0(1)$ \\
\hline
\end{tabular}





\subsubsection{TTTR an $\mathrm{LMO}_{n} / \mathrm{SMO}_{n}$}

In Abbildung 4.14 sind die temperaturabhängigen TTR-Messungen (TTTR) für die $m / n=1$ Übergitter von $\Lambda / c=2,4,6,8,10$ und 12 aufgetragen. Für die Übergit-

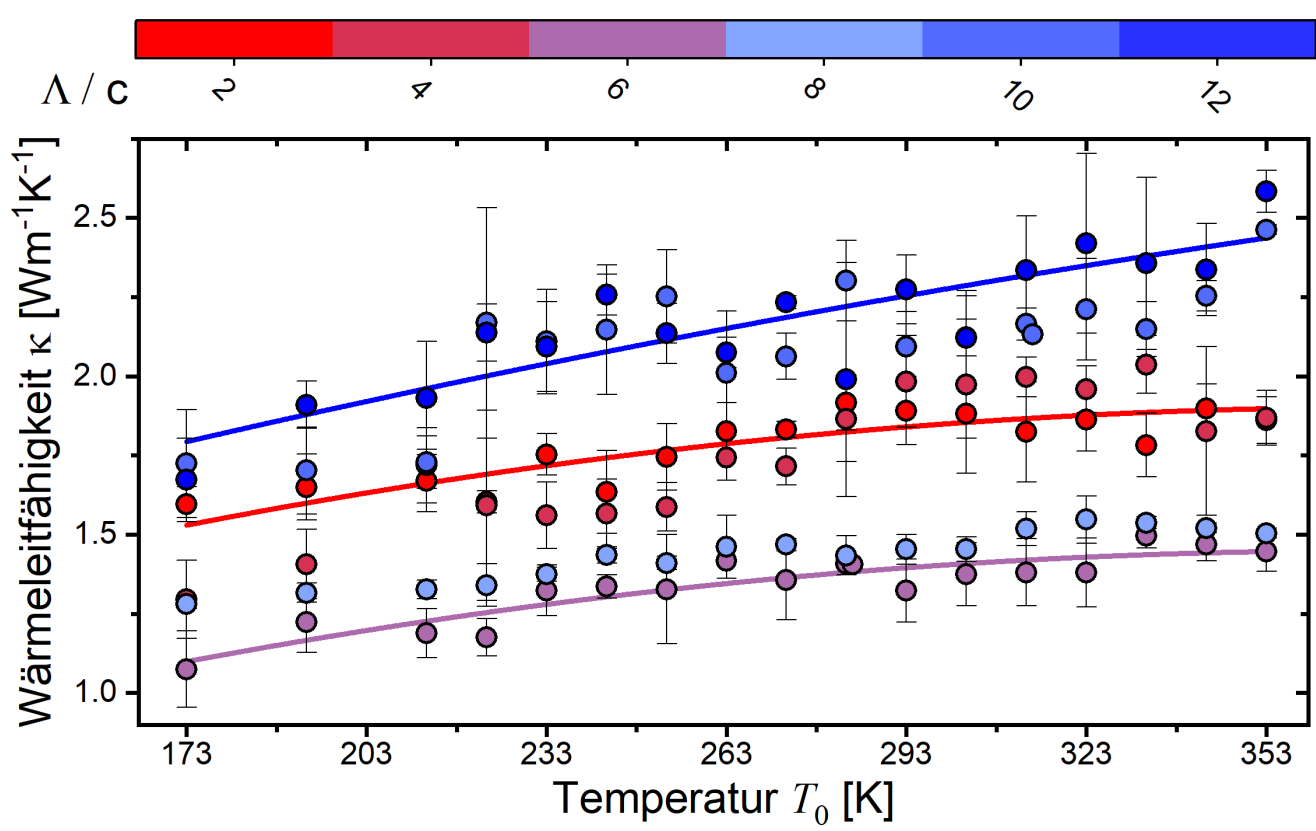

Abbildung 4.14: Temperaturabhängige TTR-Messungen an $\mathrm{LaMnO}_{3} / \mathrm{SrMnO}_{3}$ Übergittern. Die Übergitterperiode $\Lambda / c$ der Messungen ist als Farbskala dargestellt. Neben der messtechnischen Mittelung der TTR-Messungen wurden für jeden Messpunkt der Abbildung drei TTR-Kurven aufgenommen, separat ausgewertet und das Ergebnis für $\kappa$ aus diesen gemittelt. Der dargestellte Fehlerbalken ist die Standardabweichung aus dieser Mittelung. Bei den drei Linien handelt es sich um jeweils eine polynomiale Anpassung an die Messdaten der jeweiligen Farbe. Sie dienen lediglich dazu, den Trend der Messungen zu verdeutlichen.

ter mit $\Lambda / c=2,6,8$ und 10 sind zusätzlich polynomiale Kurven zweiter Ordnung als Orientierungshilfe und zur Betonung des temperaturabhängigen Trends der Messungen dargestellt. Das Ansteigen der Wärmeleitfähigkeit zu höheren Temperaturen im Bereich der Raumtemperatur wurde bereits für eine reine LMO-Schicht gemessen und wurde von Cohn et al. [109] auf eine Temperaturabhängigkeit des Grüneisenparameters $\gamma$ zurückgeführt. Für eine Analyse nutzen wir wieder das BK-Modell.

Für die Berechnung der Wärmeleitfähigkeit $\kappa$ aus den Phononenspektren des BK-Modells nutzen wir Gleichung (2.28) und ersetzen die mittlere freie Weg- 
länge durch die Relaxationszeit $\tau(q, T)=v(q) / l(q, T)$. Die Relaxationszeit der Phononen setzt sich nach dem phänomenologischen Modell von Callaway [110] und der matthiessenschen Regel aus der Relaxationszeit der einzelnen Streuprozessen zusammen[111, 112]:

$$
\frac{1}{\tau}=\frac{1}{\tau_{\mathrm{D}}}+\frac{1}{\tau_{\mathrm{U}}}
$$

Hierbei beschreibt

$$
\frac{1}{\tau_{\mathrm{D}}}=\frac{V \Gamma \omega^{4}}{4 \pi v(q)^{3}}
$$

mit dem Volumen der Einheitszelle $V$, der Gruppengeschwindigkeit $v$ und einem Faktor $\Gamma$, die Streuung der Phononen an Gitterdefekten [111, 113].

$$
\frac{1}{\tau_{\mathrm{U}}}=2 \gamma^{2} \frac{k_{\mathrm{B}} T \omega^{2}}{\mu V \omega_{\mathrm{D}}}
$$

ist die Relaxationszeit durch Phonon-Umklapp-Streuung[112]. Hier ist $\gamma=-\frac{V}{\omega} \frac{\partial \omega}{\partial V}$ der Grüneisen-Parameter als Faktor der Gitteranharmonizität [71], $\mu$ das Schubmodul und $\omega_{\mathrm{D}}$ die Debye-Frequenz. Im Rahmen unseres BK-Modells fassen wir den nicht frequenzabhängigen Anteil der Beiträge in jeweils einen Parameter zusammen:

$$
\begin{aligned}
\tau_{\mathrm{U}}(q, T) & =A(q, T) \cdot\left(\frac{k_{B}}{\hbar \omega(q)}\right)^{2} \\
\tau_{\mathrm{D}}(q) & =B(q) \cdot\left(\frac{k_{B}}{\hbar \omega(q)}\right)^{4}
\end{aligned}
$$

In Gleichung (2.28) können wir diese Faktoren und ihre Abhängigkeiten beim Berechnen der Wärmeleitfähigkeit aus den BKM-Bandstrukturen mit einbeziehen. Um die Temperaturabhängigkeit aus Abb. 4.14 genauer zu analysieren, passen wir den temperaturabhängigen Gewichtungsfaktor $A(T, q)$ an die berechneten Phononenspektren und die Messergebnisse aus dem TTR-Experiment an. $B(q)$ halten wir für diese Betrachtung konstant. Das Resultat ist in Abbildung 4.15 exemplarisch für das $\mathrm{LMO}_{3} / \mathrm{SMO}_{3}$-ÜG gezeigt. Die polynomiale Kurve $\kappa(T)$ aus Abb. 4.14 ist zum Vergleichen der Trends ebenfalls eingezeichnet. Wir erkennen: Mit steigender Temperatur nimmt $A(T, q)$ zu und damit auch die Streuzeit $\tau_{\mathrm{U}}$. Bei hohen Temperaturen begrenzt die Umklapp-Streuung den Wärmetransport in LMO/SMO-ÜG we- 


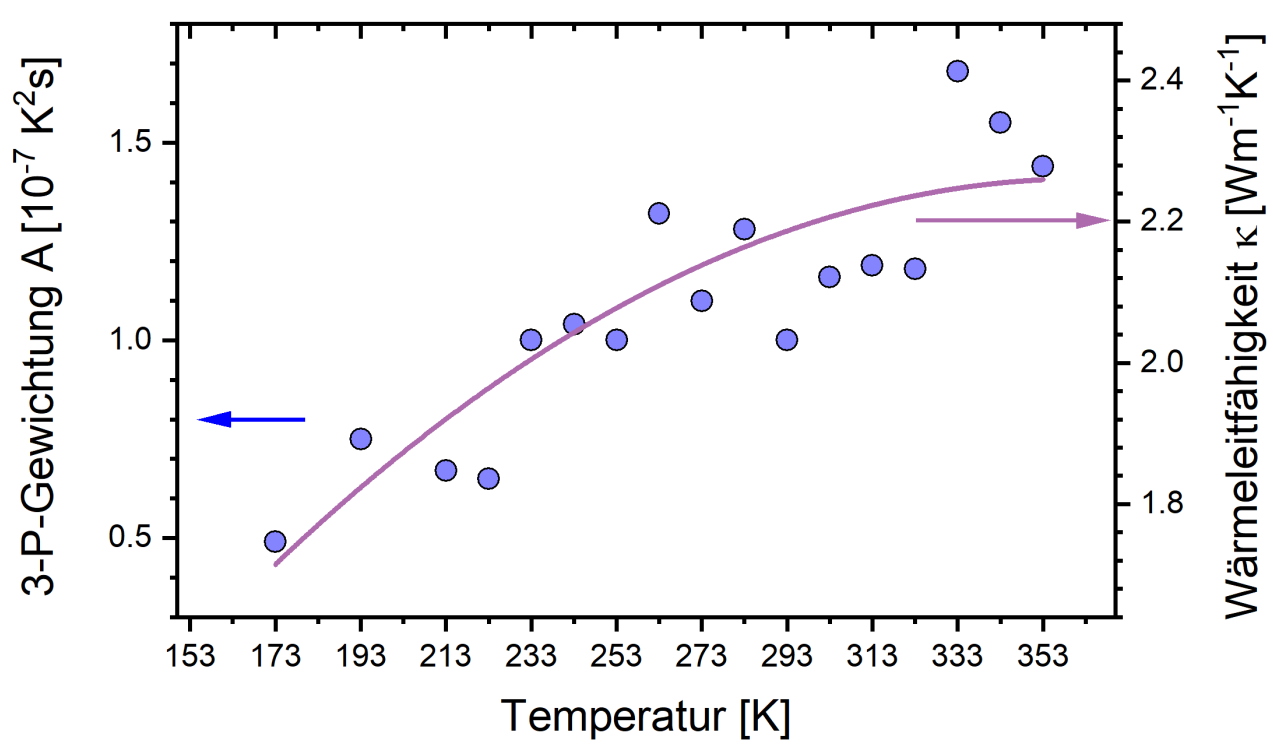

Abbildung 4.15: Durch Anpassen des BK-Modells an die gemessenen Wärmeleitfähigkeiten für $\Lambda / c=6$ ermittelte Temperaturabhängigkeit des UmklappParameters $A(T, q)$ (blau) nach Callaway [110]. $B=0.64 \times 10^{-4} \mathrm{~K}^{4}$ s wurde als nicht temperaturabhängige Größe an die Messdaten angepasst. Auf der rechten Achse ist der Trend der Wärmeleitfähigkeit der $\Lambda / c=6$ aus Abb. 4.14 eingezeichnet.

niger stark. Mit Blick auf die Definition der Relaxationszeit der Umklapp-Streuung (4.7) sehen wir, dass sich die Erklärung der anomalen Temperaturabhängigkeit von LMO (und LMO/SMO-ÜG) von Cohn et al. [109] mit unseren Messungen und unserem theoretischen Modell deckt. Wenden wir uns nun den Wärmeleitfähigkeiten als Funktion der Übergitterperiode $\Lambda / c$ zu. In Abbildung 4.16 ist dies für vier ausgewählte Temperaturen dargestellt. Wir erkennen, dass $\kappa$ von $\Lambda / c=12$ bis $\Lambda / c=6$ stetig abnimmt. Dies ist der erwartete Verlauf im Widerstandsmodell (vgl. Kapitel 4.2.1 und Gl. (4.4)). Für $\Lambda / c=2$ und 4 steigt $\kappa$ hingegen wieder an und liegt bei $\Lambda / c=2$ mit $\kappa(293 \mathrm{~K})=1.9(1) \mathrm{W} \mathrm{m}^{-1} \mathrm{~K}^{-1}$ im Bereich der Literaturwerte für $\mathrm{La}_{x} \mathrm{Sr}_{1-x} \mathrm{MnO}_{3}$ von $2.0 \mathrm{~W} \mathrm{~m}^{-1} \mathrm{~K}^{-1}$ bis $2.8 \mathrm{~W} \mathrm{~m}^{-1} \mathrm{~K}^{-1}$ [109, 114, 115] und unter dem Mittelwert der Wärmeleitfähigkeiten $\kappa_{\text {Mix }}=0.5 \cdot\left(\kappa_{\mathrm{LMO}}+\kappa_{\mathrm{SMO}}\right) \approx$ $2.9 \mathrm{~W} \mathrm{~m}^{-1} \mathrm{~K}^{-1}$. Experimentell haben wir mit dem TTR an $\mathrm{La}_{0.5} \mathrm{Sr}_{0.5} \mathrm{MnO}_{3}$ einen Wert von $\kappa_{\mathrm{LSMO}}=2.5(1) \mathrm{W} \mathrm{m}^{-1} \mathrm{~K}^{-1}$ bei Raumtemperatur gemessen. Das Minimum in der Wärmeleitfähigkeit von Übergittern wurde bereits von Simkin und 


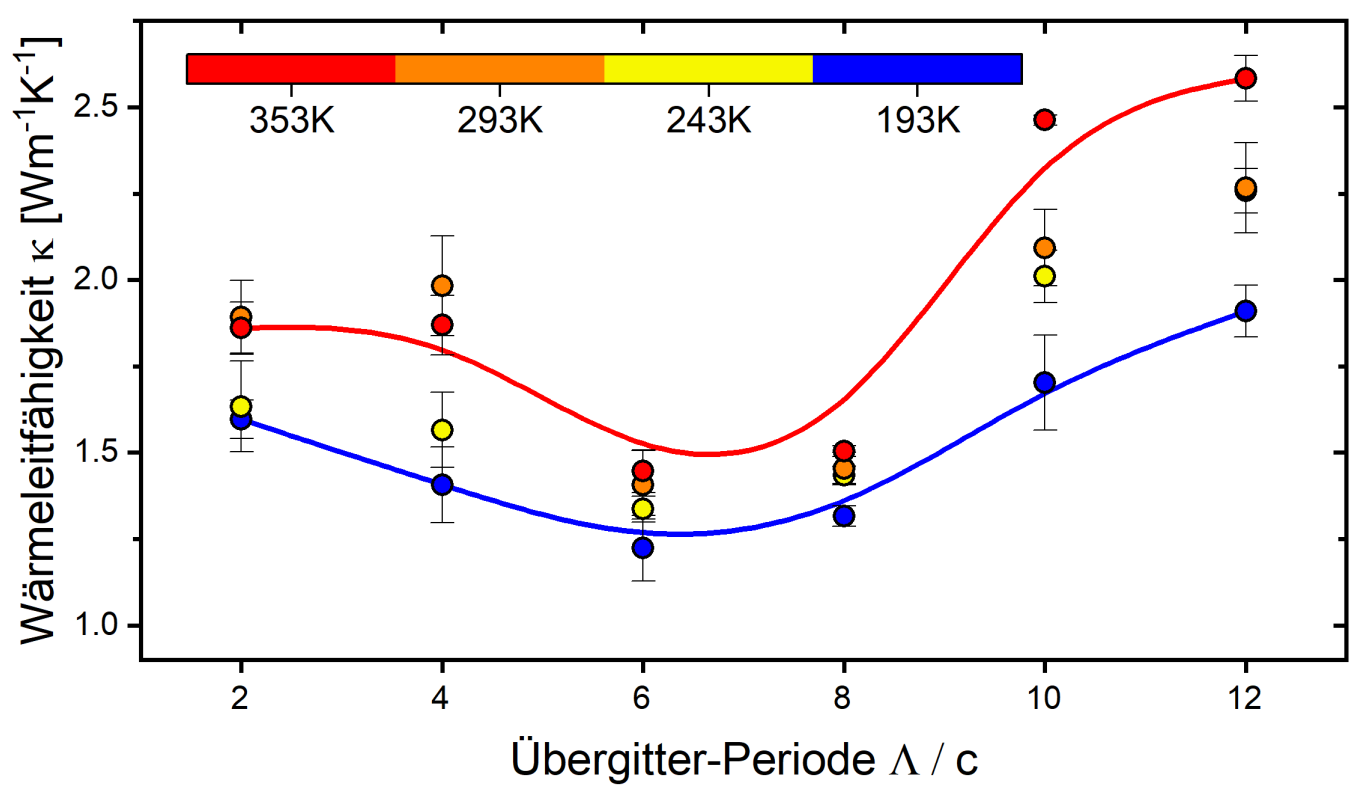

Abbildung 4.16: Wärmeleitfähigkeit von $m / n=1 \mathrm{LMO} / \mathrm{SMO}-$ Übergitter als Funktion der Übergitter-Periode $\Lambda / c$. Dargestellt sind die Messungen an vier unterschiedlichen Temperaturen. Durch die Messungen an $T=353 \mathrm{~K}$ und $T=193 \mathrm{~K}$ wurde ein Spline gelegt um den Trend der Messdaten zu veranschaulichen.

Mahan [32] berechnet und von Ravichandran et al. [39] in fast identischer Ausprägung an $\mathrm{SrTiO}_{3} / \mathrm{CaTiO}_{3}$ Übergittern gezeigt. Erneut nutzen wir unser BK-Modell um ein besseres Verständnis für die gezeigte Wärmeleitfähigkeit der Übergitter zu bekommen. In diesem Fall passen wir unser Modell mit Gl. (2.30) an das gemessene $\kappa$ an und bestimmen hieraus $\xi(T)$. Das Resultat dieser Anpassung ist für verschiedene Übergitter in Abb. 4.17 (a) dargestellt. Mit eingezeichnet sind die Bereiche in welchen die mittlere freie Weglänge $\xi / c$ einem Vielfachen der Übergitter-Periode $\Lambda / c$ entspricht. Wir stellen fest, dass für große Übergitter-Perioden $\xi \approx 3 \cdot \Lambda$ entspricht. Für das Übergitter $\Lambda / c=4$ liegt $\xi$ bei hohen Temperaturen über $4 \Lambda$ und bei niedrigen Temperaturen leicht darunter. Für $\Lambda / c=2$ liegen alle $\xi$ über $4 \Lambda$. Wir können, wie bereits in Kapitel 2.2 diskutiert, $\Lambda \sim \xi$ als den Bereich verstehen, in dem inkohärenter, ballistischer Wärmetransport in einen kohärenten Wärmetransport übergeht. In diesem kann die ÜG-Bandstruktur der Phononen nicht mehr vernachlässigt werden. Da wir in 4.17 nur eine effektive freie Weglänge der akustischen Phononen berechnen (vgl. [15]), ist es ersichtlich, dass die Grenzbedin- 

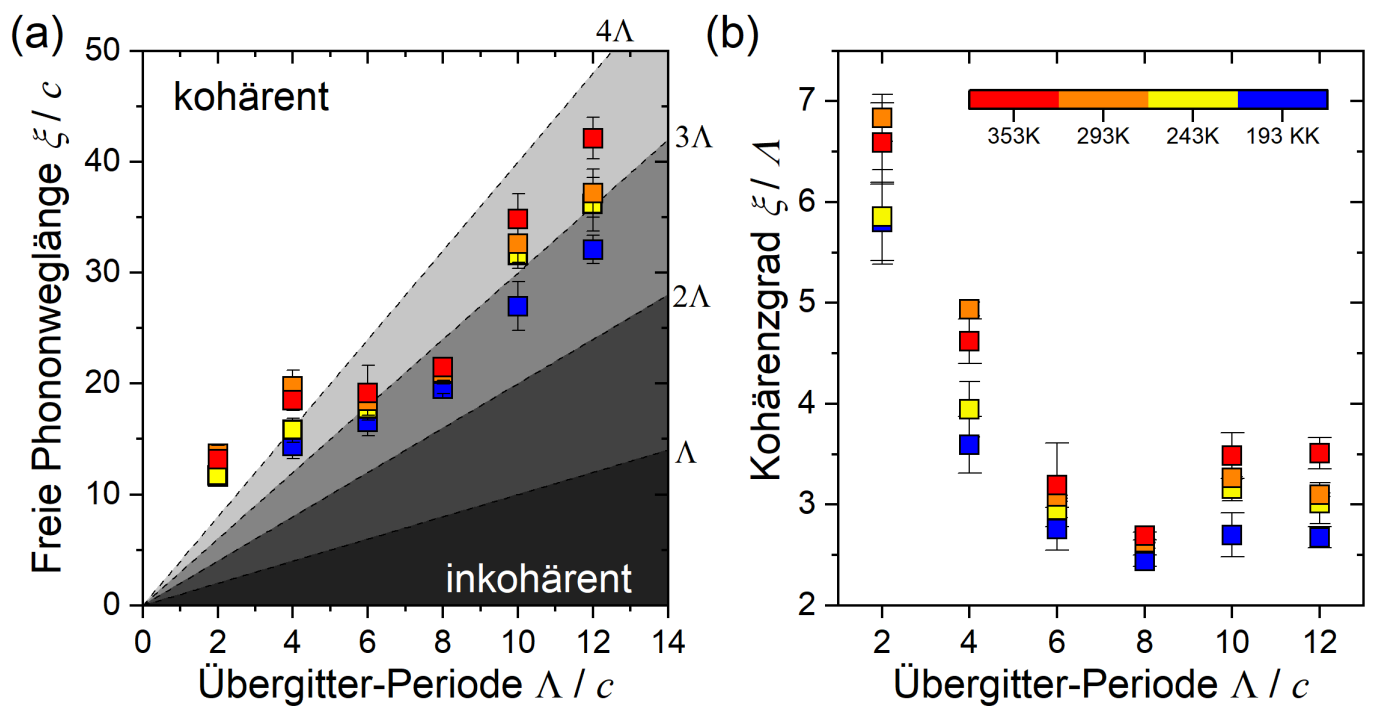

Abbildung 4.17: (a) Die mittlere freie Phononenweglänge $\xi / c$ als Funktion der Übergitterperiode $\Lambda / c$ bestimmt aus dem Anpassen von $\kappa$ aus dem BK-Modell an die TTR-Messungen. Die Temperaturskala ist dieselbe wie in (b). (b) Der Kohärenzgrad der am thermischen Transport beteiligten Phononen, definiert als $\xi / \Lambda$.

gung $\Lambda<\xi$ für kohärenten Wärmetransport keine scharfe Grenze ist, sondern der Übergang dieser beiden Bereiche des thermischen Transports fließend stattfinden. Einfach ausgedrückt: Um dies noch besser zu verdeutlichen, führe ich hier den Kohärenzgrad des phononischen Wärmetransports als $\xi / \Lambda$ ein. Dieser Wert soll uns als einfacher Anhaltspunkt für die Kohärenz der thermischen Phononen dienen. Ist der Kohärenzgrad groß, sind viele kohärente Phononen am thermischen Transport beteiligt und es zeigt sich ein ballistischer Transportcharakter. Ist der Kohärenzgrad klein, sind mehr inkohärente Phononen beteiligt und der thermische Transport wird zunehmend diffusiv. Der Kohärenzgrad der Messungen ist in Abb. 4.17 (b) als Funktion der Übergitter-Periode dargestellt. Wir sehen anhand von beiden Abbildungen 4.17 (a) und (b) sehr gut: Von dicken Übergittern kommend verhält sich die mittlere freie Weglänge $\xi$ zunächst linear als Funktion von $\Lambda$. Zwischen $\Lambda / c=6$ und 4 flacht der Trend von $\xi$ ab. In (b) erkennen wir, dass wir dies als eine Zunahme der Kohärenz des Wärmetransports verstehen können. Dieser Umkehrpunkt fällt auch mit dem Minimum und einer Trendumkehr der Wärmeleitfähigkeit als Funktion von $\Lambda$ zusammen (siehe Abb. 4.16). Ab einer Lagendicke von 3 bis 4 Atomlagen müssen wir die Welleneigenschaften der am Wärmetransport beteilig- 
ten Phononen berücksichtigen. Interferenz der Gitterschwingungen von LMO und SMO bei $\xi>\Lambda$ machen eine Beschreibung des Wärmetransports im Sinne von zwei getrennten Schichten, also zwei eigenen Bandstrukturen und den aus ihnen folgenden Materialkonstanten (z.B. $\kappa_{\mathrm{LMO}}$ und $\kappa_{\mathrm{SMO}}$ ) zwecklos. Der Wärmetransport des ÜG wird von einer eigenen Übergitterbandstruktur beschrieben. Dies lässt sich gut an den Bandstrukturen von $\Lambda / c=2,4$ und 6 erkennen, welche mit dem BK-Modell berechnet und in Abbildung 4.18 nebeneinander dargestellt sind.

(a)

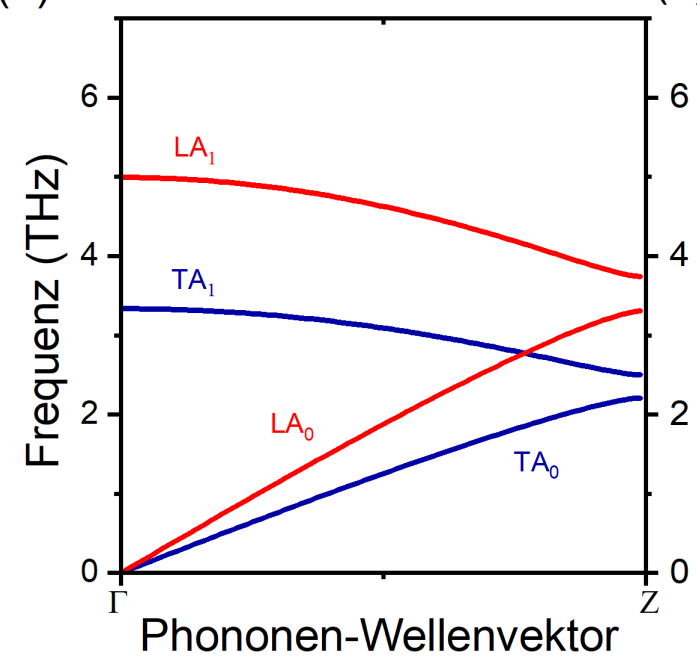

(b)

(c)

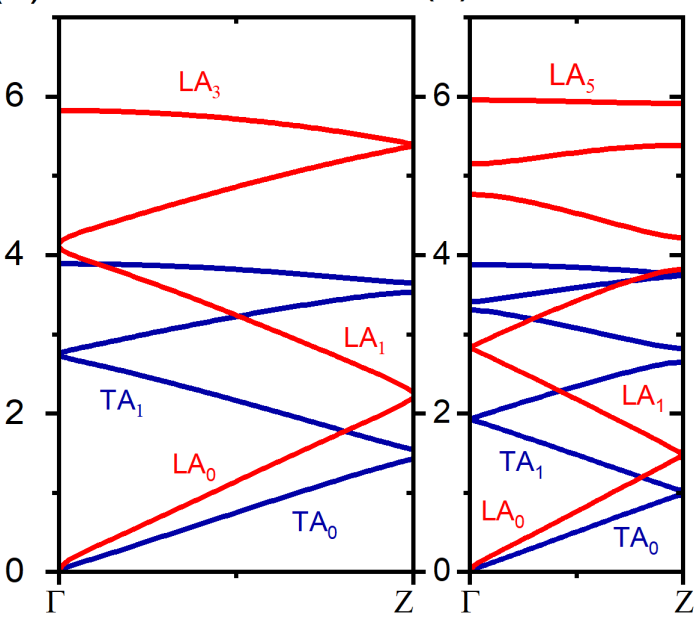

Abbildung 4.18: Mit dem BKM-Modell berechnete phononische Bandstrukturen für $\mathrm{LMO}_{1} / \mathrm{SMO}_{1}$ (a), $\mathrm{LMO}_{2} / \mathrm{SMO}_{2}$ (b) und $\mathrm{LMO}_{3} / \mathrm{SMO}_{3}$ (c) entlang der $\Gamma(0,0,0) \rightarrow Z(0,0, \pi / \Lambda)$ Achse. Longitudinale Zweige (LA) sind rot und transversale Zweige (TA) blau markiert.

In Abb. 4.18 (a) sehen wir die Bandstruktur des ÜG mit der kleinsten Einheitszelle. Der Wärmetransport wird von dem longitudinalen $\left(\mathrm{LA}_{0}\right)$ und dem zweifach entarteten transversalen Zweig $\left(\mathrm{TA}_{0}\right)$ aufgrund ihrer hoher Gruppengeschwindigkeit dominiert. Die durch Faltung an der Zonengrenze entstehenden Zweige $\mathrm{LA}_{1}$ und $\mathrm{TA}_{1}$ haben eine kleine Gruppengeschwindigkeit und tragen weniger zum Wärmetransport bei (siehe Gleichung (2.24)). Bei Verdopplung der Dicke der Doppellage (b) erhalten wir vier longitudinale und vier transversale Zweige von denen Letztere jeweils doppelt entartet sind. Die Bandlücken zwischen $\mathrm{LA}_{0}\left(\mathrm{TA}_{0}\right)$ und $\mathrm{LA}_{1}\left(\mathrm{TA}_{1}\right)$ werden hier im Vergleich mit (a) kleiner, jedoch tun sich auch neue Bandlücken auf und die Gruppengeschwindigkeit auf den einzelnen Zweigen nimmt ab. Diese 
Änderung reduziert die phononische Wärmeleitfähigkeit $(\kappa)$ im Vergleich zu (a). Dieser Trend setzt sich zu $\mathrm{LMO}_{3} / \mathrm{SMO}_{3}$ fort. Die Rückfaltung der Bänder erzeugt neue Bandlücken und die Gruppengeschwindigkeit auf den Zweigen nimmt ab. Der Effekt ist jedoch in diesem Schritt weniger groß. So hat sich im Schritt von (a) und (b) beispielsweise die Mitte der Bandlücke zwischen $\mathrm{LA}_{0}$ und $\mathrm{LA}_{1}$ von $3.5 \mathrm{GHz}$ auf $2.3 \mathrm{GHz}$ reduziert. In (c) liegt sie jedoch bei $1.5 \mathrm{GHz}$. Außerdem können wir in (c) beobachten, dass die hochfrequenten Zweige in sehr flache Äste mit niedriger Gruppengeschwindigkeit und getrennt von großen Bandlücken zerfallen. Phononen auf diesen Bändern tragen folglich nur wenig zum thermischen Transport bei. Gehen wir zu noch größeren Einheitszellen ${ }^{1}$, setzt sich der abflachende Trend durch Rückfaltung fort, jedoch ist der Effekt auf den Wärmetransport so klein, dass die Reduktion der Bandlücken an diesem Punkt die Reduktion der Gruppengeschwindigkeit kompensiert und ein Minimum der Wärmeleitfähigkeit erreicht wird.

Diese stark vereinfachte Betrachtung des Wärmetransports anhand des Phononenspektrums ist an dieser Stelle natürlich nur zur Veranschaulichung der beobachteten Effekte gedacht. Die Berechnung von $\kappa$ mit dem BK-Modell und Gleichung (2.27) (wie z.B. für Abbildung 4.17 verwendet) berücksichtigt Phononen mit Wellenvektor in alle Richtungen und bietet ein umfassenderes Bild der Abhängigkeiten (siehe dazu Abb. 4.17).

Bevor ich mich einem weiteren interessanten Effekt in den LMO/SMO Übergittern zuwende, möchte ich die Erkenntnis der bisherigen Messungen an LMO/SMO noch einmal kurz zusammenfassen:

Im Volumenmaterial oder in Multilagen lässt sich der Wärmetransport klassisch als Reihenschaltung von Wärmewiderständen beschreiben. Diese Widerstände folgen aus der materialspezifischen Wärmeleitfähigkeit und der Grenzflächenleitfähigkeit. Materialspezifische Wärmeleitfähigkeit kann auch aus dem Phononenspektrum des reinen Materials berechnet werden (vgl. Abbildung 2.14). An der Rytov-Formel (Kapitel 2.1) haben wir gesehen, dass eine akustische Fehlanpassung für das Öffnen einer Bandlücke im Übergitter verantwortlich ist und damit zu einer Barriere für die Ausbreitung elastischer Wellen führt. Dasselbe haben wir im direkten Vergleich von $m / n=1$ und $m / n=2$ Übergittern gesehen: Sind die akustischen Dispersionszweige stark verschieden (Fall $m / n=2)$, beobachten wir eine niedrige

\footnotetext{
${ }^{1}$ Weitere Spektren von $\mathrm{LMO}_{4} / \mathrm{SMO}_{4}, \mathrm{LMO}_{5} / \mathrm{SMO}_{5}$ usw. sind im Anhang A.6 abgebildet.
} 
Grenzflächenleitfähigkeit. Sind die akustischen Dispersionszweige ähnlich, sehen wir im Fall $m / n=1$ dass die Grenzflächenleitfähigkeit um mehr als das Vierfache zunimmt.

In Übergittern mit kleiner Übergitter-Periode und großer freier Weglänge der Phononen können wir das Phononenspektrum der einzelnen Materialien nicht mehr trennen und materialspezifische Wärme- und Grenzflächenleitfähigkeiten verlieren ihre Rechtfertigung. Es lässt sich nur noch ein neues $\kappa$ für das gesamte Übergitter definieren. Dieses kann mit Hilfe der ÜG-Bandstruktur und Gl. (2.30) berechnet werden.

\subsubsection{Wärmetransport in LMO/SMO mit AFM-Phase}

Das Auftreten eines komplexen Zusammenspiels von Ferro- und Antiferromagnetismus an den Grenzflächen von LMO/SMO Übergittern wurde 2020 von Keunecke et al. gezeigt [85]. Einen direkten Einfluss der magnetischen Eigenschaften der Übergitter auf den phononischen Wärmetransport konnte in den Messungen im vorherigen Kapitel an idealen LMO/SMO Übergittern nicht gefunden werden. Jedoch zeigt die magnetometrische Messung an einer $\Lambda / c=4$ Probe mit leichter Variation der Wachstumsparameter $(+10 \%$ SMO und $-10 \%$ LMO in der Gesamtstöchiometrie) eine starke Abnahme des magnetischen Momentes. Dies ist in Abbildung 4.19 dargestellt. Die Reduktion des magnetischen Momentes des variierten Übergitters (dunkelrot) im Vergleich zum idealen Übergitter (hellrot) können wir durch einen erhöhten Beitrag der AFM-Phase zur Gesamtmagnetisierung verstehen. Gestützt wird diese Annahme durch einen Anstieg der Magnetisierung bei etwa $T_{\mathrm{AFM}}=323 \mathrm{~K}$ (im Inset vergrößert dargestellt). Wir interpretieren $T_{\mathrm{AFM}}$ als die Temperatur an welcher die AFM-Phase verschwindet, wodurch sich der FM-Beitrag kurzzeitig verstärkt.

In Abbildung 4.20 sind nun TTR-Messungen als Funktion der Temperatur für das Übergitter mit erhöhtem AFM-Beitrag dargestellt. Die eingezeichneten Trends für die $\ddot{\mathrm{UG}} \Lambda / c=2,6$ und 12 sind identisch mit denen in Abbildung 4.14. Wie gut $\mathrm{zu}$ erkennen ist, liegen die Wärmeleitfähigkeiten der AFM-Probe bei fast allen Temperaturen fast $50 \%$ unter der vergleichbaren $\Lambda / c=4$ Probe (rote Linie). Für Temperaturen über $T_{\mathrm{AFM}}$ strebt die Wärmeleitfähigkeit gegen den Wert, den wir auch für das ideale Übergitter $\Lambda / c=4$ gemessen haben. Dies ist ein starker Hinweis 


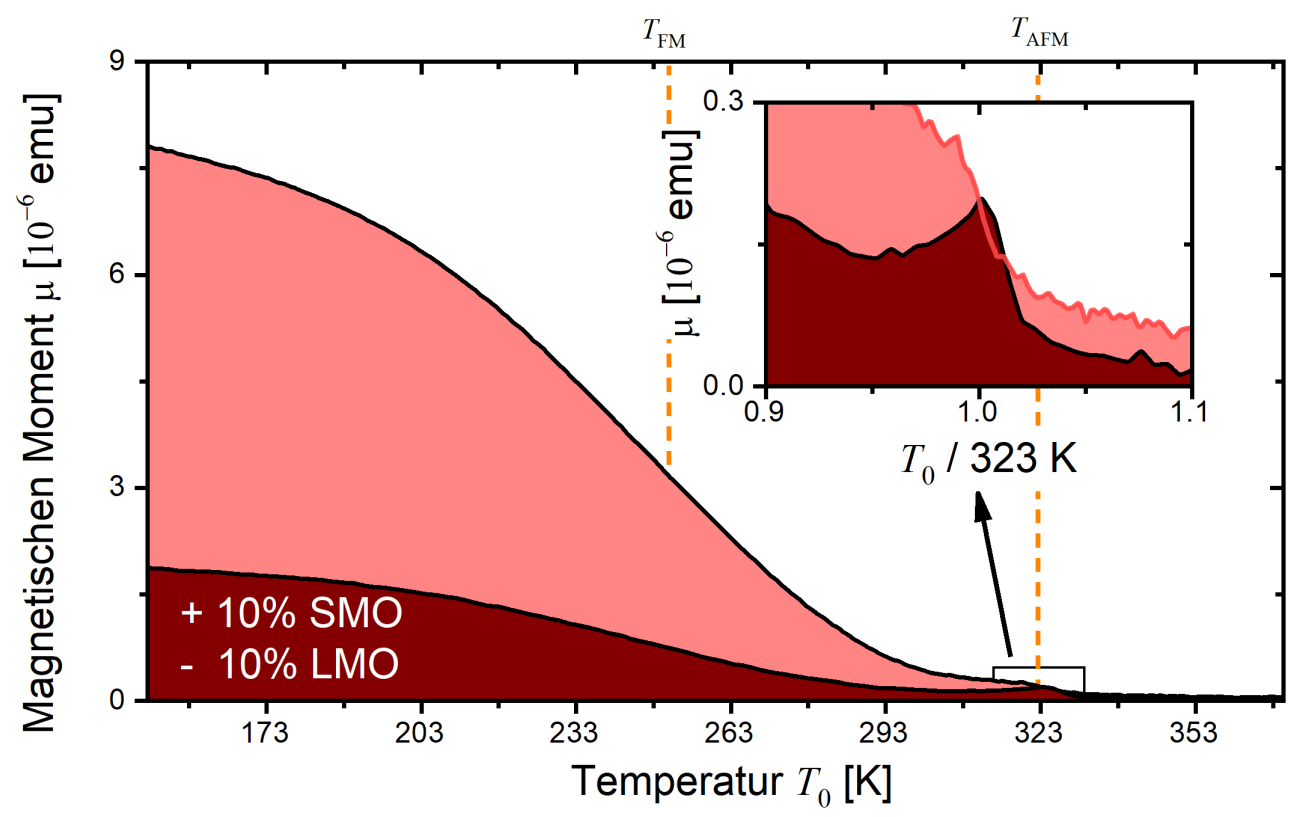

Abbildung 4.19: Magnetisches Moment bei $10 \mathrm{mT}$ als Funktion der Temperatur für ein Übergitter mit $\Lambda / c=4$ mit idealer Stöchiometrie (hellrot) und einem mit 10\% mehr SMO und 10\% weniger LMO in der Gesamtstöchiometrie des Übergitters (dunkelrot) gemessen. Im Inset ist ein Bereich um $323 \mathrm{~K}$ vergrößert dargestellt.

darauf, dass die Reduktion der Wärmeleitfähigkeit um knapp 50 \% nur eine indirekte Folge der Änderung der Wachstumsparameter ist, aber eine direkte Folge des Auftretens der verstärkten AFM-Kopplung in dieser Probe. Im Bereich von etwa $233 \mathrm{~K}$ bis $283 \mathrm{~K}$ sehen wir von niedrigen Temperaturen kommend, zunächst einen starken Anstieg der Wärmeleitfähigkeit, gefolgt von einem ähnlich starken Abfall. Es sei an dieser Stelle noch einmal darauf hingewiesen, dass sowohl $\kappa$ als auch $C_{p}$ freie Parameter in der Auswertung des TTR sind. Mit Blick auf die Magnetisierungsmessungen (Abb. 4.19) sehen wir, dass wir in diesem Temperaturbereich gerade im Bereich der stärksten Änderung der Magnetisierung der Probe sind. Ein Einfluss der magnetischen Ordnung der Probe auf die spezifische Wärme in diesem Bereich ist daher naheliegend, jedoch bis zu diesem Zeitpunkt ungeklärt. Zukünftige magnetfeldabhängige TTR- oder $3 \omega$-Messungen sowie kalorimetrische Messungen bieten sich daher für die genauere Untersuchung dieses Bereichs der Probe mit AFM-Kopplung an. 


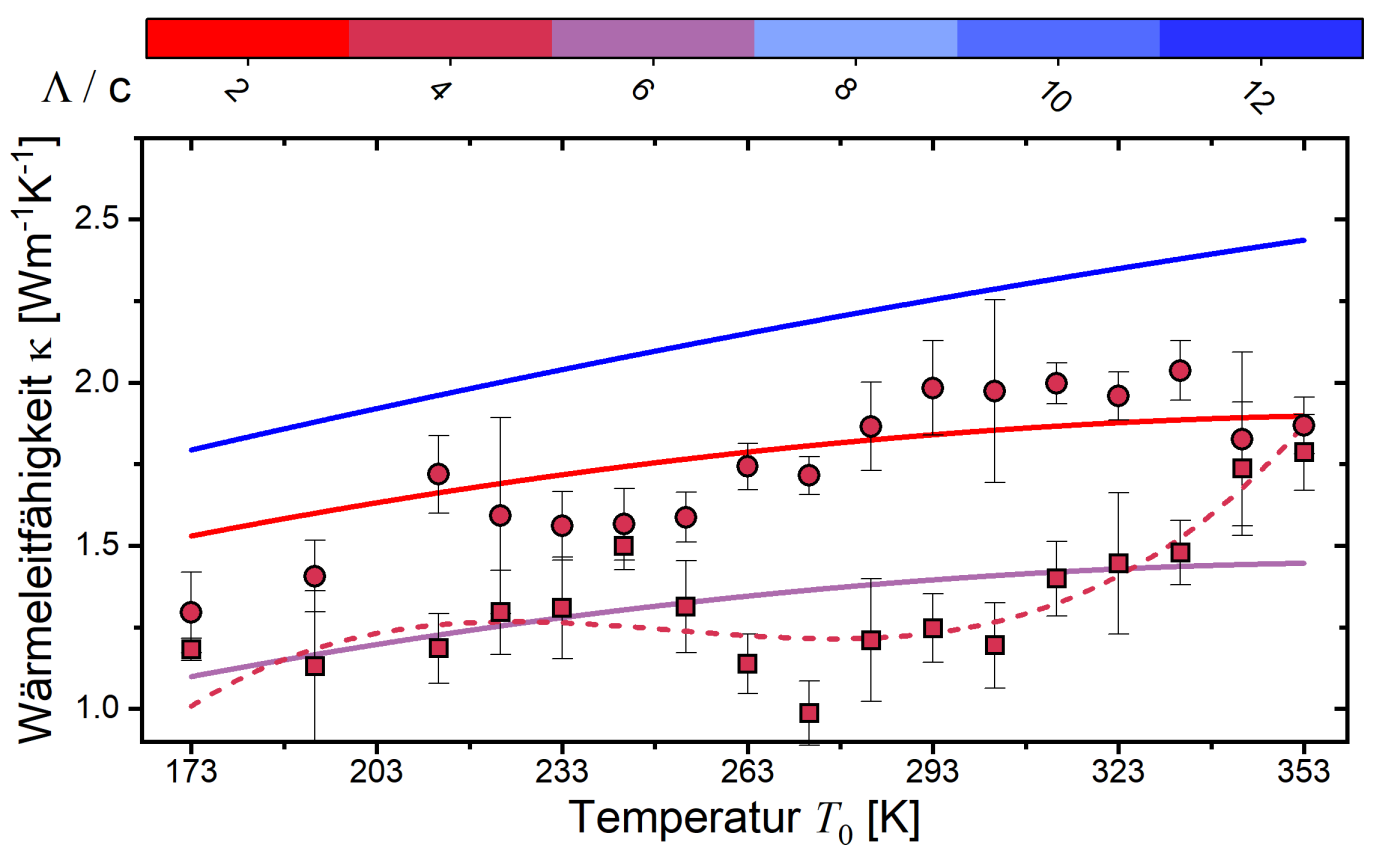

Abbildung 4.20: Mit dem TTR gemessene Wärmeleitfähigkeit $\kappa$ der $\Lambda / c=4$ Probem mit idealer Stöchiometrie (Kreis) und abweichender Stöchiometrie (Quadrat). Außerdem dargestellt sind die angepassten Trendkurven $\Lambda / c=4,6$ und 12, wie in Abb. 4.14. Die gestrichelte Linie soll den Trend der quadratischen Messpunkte verdeutlichen.

Erneut berechnen wir mit unserem BK-Modell die mittlere freie Weglänge der am thermischen Transport beteiligten Phononen und bestimmen daraus den Kohärenzgrad $\xi / \Lambda$. Dies ist für beide $\Lambda / c=4$ Proben in Abbildung 4.21 dargestellt. Wir stellen für die Probe mit AFM-Kopplung fest, dass auch ungeachtet des lokalen Maximums bei $T=243 \mathrm{~K}$ (knapp unter $T_{\mathrm{FM}}=250 \mathrm{~K}$ ) der Kohärenzgrad der Phononen dieser Probe für den gesamten Temperaturbereich unter dem der Probe mit dominierender FM-Kopplung liegt. Für die Temperaturen $343 \mathrm{~K}$ und $353 \mathrm{~K}$, nur wenig über $T_{\mathrm{AFM}}=323 \mathrm{~K}$, und für die tiefeste Temperature $173 \mathrm{~K}$ ist der Kohärenzgrad und die Wärmeleitfähigkeit (Vergleich von Abb. 4.14 und 4.20) nahezu gleich.

Eine mögliche Erklärung wäre, dass Magnonen und Phononen hybridisieren und neue Magnon-Polaronen-Zustände [116] bilden. Diese Zustände würden als zusätzlicher Streukanal die mittlere freie Weglänge der Phononen herabsetzen und dadurch die Reduktion der Wärmeleitfähigkeit in Anwesenheit der AFM-Phase 


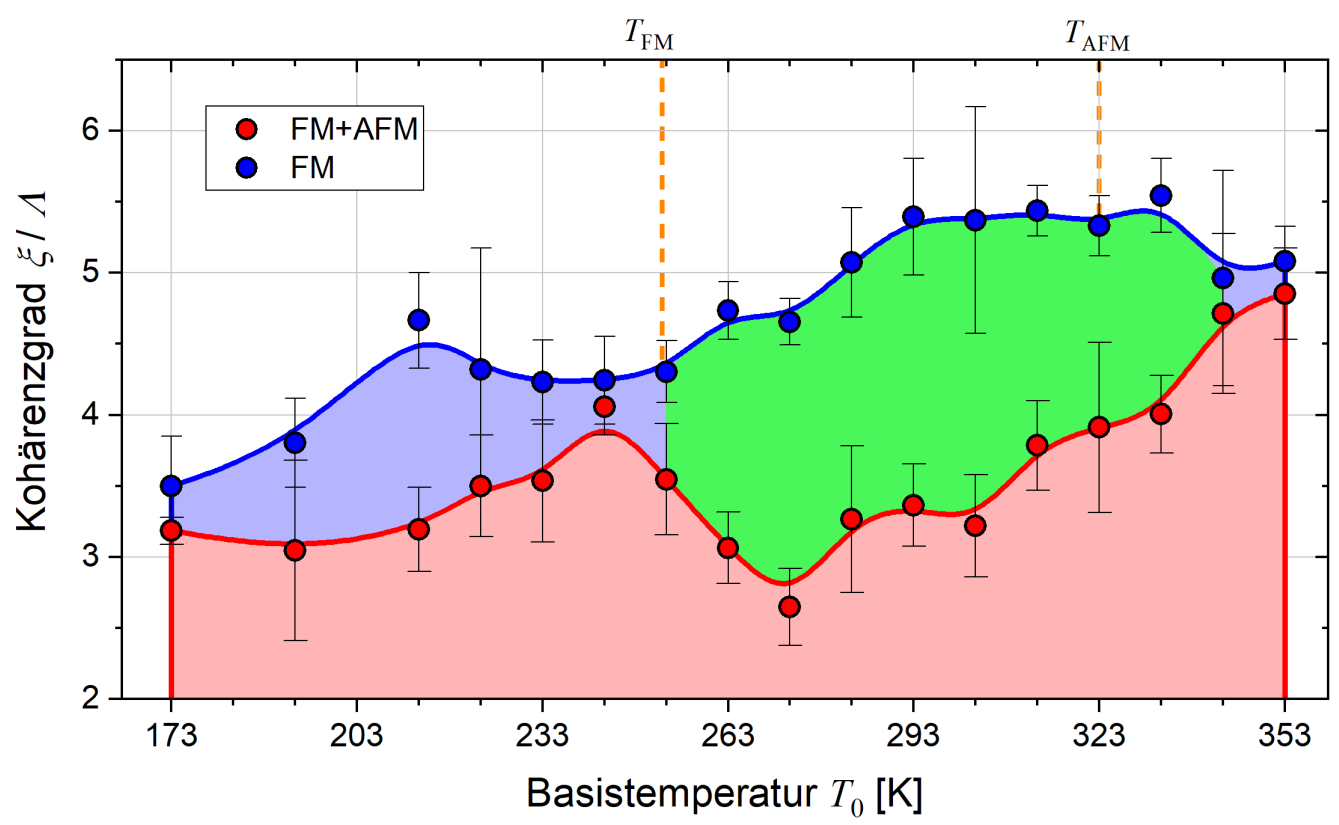

Abbildung 4.21: Vergleich des Kohärenzgrades $\xi / \Lambda$ der $\Lambda / c=4$ Proben mit starker AFM-Kopplung (rot) und mit dominierender FM-Kopplung (blau). Grün hervor gehoben ist ein Bereich zwischen $T_{\mathrm{FM}}$ und $T=343 \mathrm{~K}$ in dem sich der Kohärenzgrad zwischen den beiden Proben besonders stark unterscheidet.

erklären. Der Einfluss einer antiferromagnetischen Phase auf die Dämpfung von Ultraschall ist bereits bekannt [117, 118]. Im Falle einer periodischen Verteilung der Bereiche mit Phonon-Magnon-Hybridisierung in der Probe (z.B. an jeder Grenzfläche innerhalb des Übergitters), würde diese zur Öffnung neuer Bandlücken im Phononespektrum führen. Der Einfluss einer solchen Kopplung auf das Phononenspektrum wurde bereits in den 70ern theoretisch beschrieben [119] und Hinweise auf die so erzeugten Bandlücken wurden bereits an KCl-CN Kristallen mittels inelastischer Neutronenstreuung gefunden [120]. Wie bereits im Kapitel 2.2.2 und von Simkin und Mahan gezeigt [32] führt das Öffnen neuer Bandlücken zu einer Reduktion der Wärmeleitfähigkeit. Mit typischen Spinwellenfrequenzen von $1 \mathrm{THz}$ [121], liegt die neue Bandlücke in einem für den thermischen Transport wichtigen Frequenzbereich. Die Änderung des Kohärenzgrades bzw. der mittleren freien Weglänge kann so erklärt werden, dass unser BK-Modell keine zusätzlichen Bandlücken im Phononenspektrum berücksichtigt. Das Anpassen unseres Modells führt so zu einer unterschätzten mittleren freien Weglänge. Trotzdem sehen wir durch 
Anpassen des Modells und Bestimmung des Kohärenzgrades in Abb. 4.21 zwischen $T_{\mathrm{FM}}=250 \mathrm{~K}$ und $T_{\mathrm{AFM}}=323 \mathrm{~K}$ (grün) einen Bereich, in dem sich die Kohärenz des thermischen Transporten in den beiden Proben deutlich unterscheidet.

Um die Ursache der Reduktion von $\kappa$ genauer zu untersuchen, sind jedoch weitere Messungen nötig, z.B. magnetfeldabhängige TTR-Messungen. Ein kritisches, externes Feld sollte die Probe auch bei Temperaturen unter $T_{\mathrm{AFM}}$ von der FM+AFMPhase in eine reine FM-Phase bringen. Im Einklang mit den obigen Erklärungen sollte dann die Wärmeleitfähigkeit der Probe ansteigen. Dies wäre ein weiterer starker Hinweis darauf, dass die Reduktion von $\kappa$ in dieser Probe mit dem Magnetismus in Verbindung steht und nicht etwa mit erhöhter Streuung an Grenzflächen oder Defekten aufgrund der leicht unterschiedlichen Wachstumsparameter. Solche Messungen konnte ich aufgrund zeitlicher Limitierung und den hierzu nötigen Umbauten am TTR-Experiment leider nicht mehr umsetzen. Weitere Gedanken zu möglichen zukünftigen Messungen werde ich im Ausblick meiner Dissertation notieren. 


\section{Kapitel 5}

\section{Zusammenfassung und Ausblick}

In diesem Kapitel möchte ich die Erkenntnisse aus meiner Dissertation noch einmal zusammengefasst darstellen. Darüber hinaus möchte ich in einem Ausblick Ansatzpunkte für weitere Messungen vorstellen, die dazu genutzt werden könnten, die Untersuchung des Wärmetransportes und speziell auch der magnetoelastischen Kopplung in LMO/SMO-Übergittern fortzusetzen.

Auch möchte ich in einem Unterkapitel meine Bemühungen zum Koppeln der elastischen Dynamik an eine magnetische Oszillation vorstellen und die möglichen Fehler in meinem Ansatz darstellen.

\subsection{Ultraschalldynamik in Multilagen}

Mit der Herleitung der Rytov-Formel für eine Multilage und der Erweiterung für eine Multilage mit Deckschicht habe ich in Kapitel 2.1 gezeigt, dass wir die akustischen Bandlücken in einer Multilage vorab berechnen können und dass durch das Abstimmen von Resonanzfrequenz der Deckschicht und der Bandlücke eine schwach gedämpfte Oberflächenmode zu erwarten ist.

Mit den Pump-Probe-Reflextions-Messungen in Kapitel 4.1.1 haben wir diese Oberflächenmode über eine Änderung der Reflektivität der Wolframschicht auf zwei unterschiedlichen $\mathrm{MgO} / \mathrm{ZrO}_{2}$-Übergittern nachgewiesen. Durch eine Variation der Wolframschichtdicke konnten wir diese darüber hinaus nutzen, um die Bandlücke der Multilage zu vermessen und so die Vorhersagen der Rytov-Formel zu bestätigen. Mit einer Halbierung der Doppellagendicke hat sich die Frequenz der ersten Bandlücke wie erwartet in etwa verdoppelt. 
Mit Hilfe der Finite-Differenzen-Simulation 4.1.2 haben wir zusätzlich einen Einblick in die (theoretische) elastische Dynamik im Inneren der Probe erlangt, welcher uns im Reflektivitäts-Experiment normalerweise verborgen bleibt.

Aus den Messungen, Simulationen und Berechnungen können wir festhalten, dass die Kontrolle über die Zusammensetzung und Nanostrukturierung einer solchen Probe genutzt werden kann, um bestimmte Frequenzen im elastischen Spektrum an der Struktur zu reflektieren, während andere Frequenzen sie noch ungehindert passieren können.

Auch wenn wir mit den Multilagen erst den niederfrequenten Bereich des Spektrums der elastischen Dynamik $(<1 \mathrm{THz})$ erschlossen haben, konnten wir hier bereits sehen, dass wir durch die periodische Strukturierung einer Probe einen Teil der elastischen Energie an der Transmission durch die Struktur hindern können um sie beispielsweise in einer Oberflächenmode zu lokalisieren.

Bevor ich mich in dieser Zusammenfassung dem Wärmetransport widme, möchte ich im folgenden Kapitel noch kurz das Thema im Zusammenhang mit der Ultraschalldynamik ansprechen, welches ich leider in der mir gegebenen Zeit nicht mehr abschließen konnte.

\subsection{Magnetoelastische Kopplung in LSMO/(LMO/SMO)}

Neben der Analyse der Bandlücke war es eine weitere Idee, die Oberflächenmode in Multilagen mit Deckschicht (Kapitel 4.1) zur Erzeugung hochfrequenter Spinwellen zu nutzen. Diese Idee möchte in den folgenden beiden Unterkapiteln anhand von Vorüberlegungen und mikromagnetischen Simulationen veranschaulichen.

Wie wir bereits wissen, entspricht die Frequenz der Oberflächenmode $f_{\mathrm{A}}$ in einer angepassten Multilage mit Deckschicht der Dicke $d$

$$
f_{\mathrm{A}}=\frac{v_{\mathrm{L}}}{2 d}
$$

Die Frequenz einer Spinwelle $f_{\mathrm{S}}$ ist in Abhängigkeit der Schichtdicke $d$, der Ordnung der Spinwelle $n_{\mathrm{S}}$ und dem externen magnetischen Feld $H$ in erster Näherung [26]

$$
f_{\mathrm{S}}=\gamma \frac{A \pi n_{\mathrm{S}}^{2}}{M_{\mathrm{S}} d^{2}}+\gamma \frac{\mu_{0} H}{2 \pi}
$$


mit der Austauschkonstante $A$, der Sättigungsmagnetisierung $M_{\mathrm{S}}$ und dem gyromagnetischen Verhältnis $\gamma$. Für die resonante Kopplung zwischen akustischer und magnetischer Oszillation innerhalb eines Films gilt folglich:

$$
\frac{v_{\mathrm{L}}}{2 d}=\gamma \frac{A \pi n_{\mathrm{S}}^{2}}{M_{\mathrm{S}} d^{2}}+\gamma \frac{\mu_{0} H}{2 \pi}
$$

Diese Bedingung schränkt die Material- und Frequenzwahl sehr stark ein, da es nur eine Schichtdicke für jedes Material gibt, an denen die Frequenzen von magnetischer und akustischer Mode überlappen. Einsetzen von typischen Materialparametern in Gl. 5.3 zeigt zudem, dass die resonante Schichtdicke für magnetische Deckschichten wie beispielsweise Cobalt-Eisen-Bor (CoFeB) bei $6 \mathrm{~nm}$ und damit auch bei einer relativ hohen Resonanzfrequenz von $450 \mathrm{GHz}$ liegt. Eine weitere Idee war es deshalb, eine Deckschicht aus einer Wolfram-LSMO oder WolframCoFeB Doppellage auf einer Multilagenprobe herzustellen. In diesem Fall könnte die akustische Resonanz ohne Rücksicht auf Spinwellenfrequenz auf die Bandlücke der Multilage angepasst werden. Daraufhin kann dann die Schichtdicke des magnetischen Films auf die Dicke für eine magnetische Resonanz eingestellt werden.

Um vorab zu prüfen, inwieweit eine akustische Oberflächenmode im GHz-Bereich geeignet ist, eine magnetische Oszillation anzuregen, haben wir das zeitliche Profil der Oszillation bei $15.1 \mathrm{~nm}$ Wolframdicke aus Abb. 4.3 genommen und als elastische Anregung für eine CoFeB-Schicht in eine Simulation mit dem Programm MuMax [122] eingeben. Die Schichtdicke dieser CoFeB-Schicht haben wir so angepasst, dass die akustische Resonanzfrequenz der Anregung von $182 \mathrm{GHz}$ entspricht. Das externe Feld $\mu_{0} H_{\text {Ext }}=600 \mathrm{mT}$ wurde so gewählt, dass die Resonanzbedingung 5.3 für $n_{\mathrm{S}}=2$ erfüllt ist. Die Wahl von $n_{\mathrm{S}}=2$ folgt auf Grund von einer Symmetriebetrachtung: Die akustische Mode erster Ordnung hat je einen Schwingungsknoten an der Probenoberfläche und der Grenzfläche zwischen Schicht und Multilage. Die größte Amplitude dieser Schwingung ist in der Mitte des Films (siehe z.B. Abb. 4.7). Die Schwingungsknoten einer magnetischen Oszillation hingegen liegen im Inneren der Schicht, mit der 0. Ordnung (FMR-Mode) ohne Schwingungsknoten, der 1. Ordnung mit einem Knoten in der Mitte des Films, der 2. Ordnung mit zwei Knoten auf $1 / 4$ und 3/4 der Schichtdicke, usw. Demnach findet die stärkste Kopp- 
lung zwischen akustischer Welle (1. Ordnung) und Spinwelle 0. und 2. Ordnung in der Mitte der Probe statt.

In Abbildung 5.1 ist die Anregungsdynamik (a) und die resultierende magnetische Dynamik (b) aus der mikromagnetischen Simulation $\operatorname{MuMax}^{3}[122]$ dargestellt.

(a)
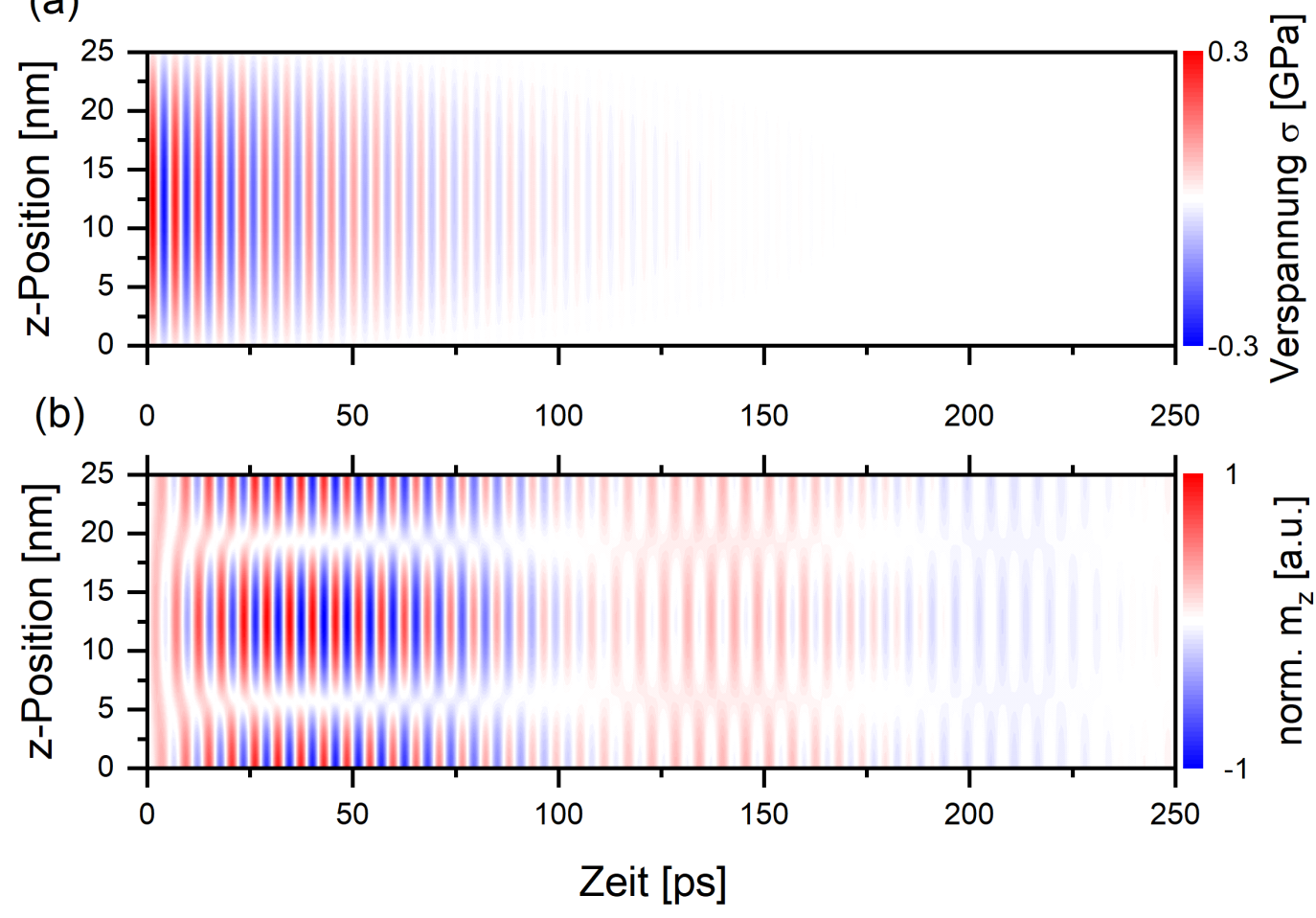

Abbildung 5.1: (a) Zeit- und Ortsprofil der akustischen Verspannung, welche wir als dynamische Anregung für die mikromagnetische Simulation nutzen. (b) Zeit- und Ortsprofil der Magnetisierung $\left(m_{\mathrm{Z}}\right)$ in z-Richtung, welche wir mit Hilfe der dynamischen Anregung aus der mikromagnetischen Simulation MuMax ${ }^{3}$ [122] gewonnen haben.

In der Tat sehen wir in der magnetischen Simulation (b) nach einem kurzen Einschwingvorgang von etwa 35 ps die Ausbildung einer magnetischen Oszillation mit dem Profil einer Mode 2. Ordnung und einer Frequenz von 180(1) GHz. Im Hintergrund ist eine FMR-Mode mit einer Frequenz von wenigen $\mathrm{GHz}$ zu beobachten. Eine magnetische Oszillation 1. Ordnung (Wellenknoten in der Mitte der Schicht) ist wie erwartet nicht zu sehen. 
Aufgrund der Verfügbarkeit von LMO/SMO-Proben haben wir uns für die Demonstration der magnetoelastischen Kopplung für eine Multilage aus LMO/SMO und einer magnetischen Schicht aus LSMO entschieden. In ersten Testmessung konnte, vermutlich durch die Wolfram-Deckschicht, kein magnetisches Signal gemessen werden.

Eine weitere Probenreihe aus CoFeB-Schicht und LMO/SMO-ÜG wurde hergestellt aber aufgrund der Sperrung der Universität im Zuge der aufkommenden Pandemie im Frühjahr 2020 leider nicht mehr vermessen.

\subsection{Wärmetransport in Übergittern}

Um den Einfluss der Bandstruktur eines Übergitters auf den Wärmetransport zu untersuchen, habe ich in Kapitel 2.2.2 eine auf den Vorarbeiten von Simkin und Mahan [32] und Ziman [31] basierende Beschreibung der phononischen Wärmeleitung als Funktion der mittleren freien Weglänge der Phononen und ihrem Dispersionspektrum vorgestellt. Dieses Modell beschreibt nicht nur die Wärmeleitfähigkeit $\kappa$ von nicht dielektrischen Festkörpern als Funktion ihrer phononischen Bandstruktur, es erlaubt auch die Beschreibung der erwarteten Wärmeleitfähigkeit eines Übergitters aus unterschiedlichen Materialien. Damit liefert es uns das theoretische Werkzeug, die Erkenntnisse aus den Experimenten an $\mathrm{MgO} / \mathrm{ZrO}_{2^{-}}$ Multilagen (4.1.1) auf Größenordnung von typischen Gitterkonstanten zu skalieren. Damit können wir das Konzept, den elastischen Energietransport durch das Erzeugen künstlicher Bandlücken zu beeinflussen in den spektralen Bereich von THz-Phononen und damit in den Bereich des Wärmetransportes bringen.

Mit dem TTR-Aufbau (3.4.1) habe ich dann auch einen experimentellen Zugang zum Wärmetransport in Übergittern vorgestellt. Die Leistungsfähigkeit des Aufbaus und unserer Auswertungsroutine (Kapitel 3.4.1.2) der Messdaten habe ich anhand von $\mathrm{ZrO}_{2}$-Testproben demonstriert. Dieselben Proben wurden bereits in der ersten Förderperiode des SFB zur Beurteilung der Leistungsfähigkeit eines ähnlichen Aufbaus genutzt. Der direkte Vergleich der ersten und zweiten Förderperiode verdeutlicht die Fortschritte die wir mit unserem Experiment erreicht haben. 
Am Vergleich der Wärmeleitfähigkeit von $m / n=2$ und $m / n=1 \mathrm{LMO} / \mathrm{SMO}$ Übergittern konnten wir ableiten, dass die zuvor beobachtete [80] Eigenschaft des LMO in $m / n=2$ in rhomboedrischer Struktur und in $m / n=1$ mit der kubischen Struktur (Struktur der SMO-Schichten) einen großen Einfluss auf die Wärmeleitfähigkeit des Übergitters hat. Die Volumenwärmeleitfähigkeit der $m / n=2$ Proben mit $\kappa_{\mathrm{V}}=2.4(4) \mathrm{W} \mathrm{m}^{-1} \mathrm{~K}^{-1}$ liegt nahe am Mittelwert der Wärmeleitfähigkeit $\kappa_{\mathrm{Mix}} \approx 2.7 \mathrm{~W} \mathrm{~m}^{-1} \mathrm{~K}^{-1}$ seiner Bestandteile liegt $\left(\kappa_{\mathrm{LMO}}=1.3(2) \mathrm{W} \mathrm{m}^{-1} \mathrm{~K}^{-1}\right.$ und $\kappa_{\mathrm{SMO}}=4.5 \mathrm{~W} \mathrm{~m}^{-1} \mathrm{~K}^{-1}$ [101]). Die Wärmeleitfähigkeit der $m / n=1$ Übergitter mit $\kappa=5.6(26) \mathrm{W} \mathrm{m}^{-1} \mathrm{~K}^{-1}$ ist etwa dreimal so hoch. Auch die Grenzflächenleitfähigkeit steigt in $m / n=1 \mathrm{um}$ fast das Fünffache an und liegt mit $h=$ 1.6(3) $\mathrm{GW} \mathrm{m}^{-2} \mathrm{~K}^{-1}$ deutlich über üblichen Werten für Isolator-Isolator-Grenzflächen von $<1 \mathrm{GW} \mathrm{m}^{-2} \mathrm{~K}^{-1}$ [123-125]. Es liegt sogar im Bereich der Rekordgrenzflächenleitfähigkeit der metallischen Al-Cu-Grenzflächen von $h_{\mathrm{Cu}-\mathrm{Al}}=4 \mathrm{GW} \mathrm{m}^{-2} \mathrm{~K}^{-1}$ [126].

Diese Ergebnisse können wir als ersten Hinweis sehen, dass die Gitterstruktur und damit die Bandstruktur der Phononen auf der Größenskala unserer Übergitter eine bedeutende Rolle spielt. Deshalb haben wir uns eine weitere Reihe $m / n=1$-Proben mit Hilfe des TTR-Experimentes angesehen. Dass dabei LMO und SMO in der gleichen, kubischen Struktur vorliegt macht diese Übergitter zu einem ausgezeichneten Modell-System um den Einfluss kohärenter Phononen und der Bandstruktur auf den Wärmetransport zu beobachten. Und so haben wir an LMO/SMO-Übergittern den von Simkin und Mahan berechneten [32] und bereits an CTO/STO-ÜG von Ravichandran et al. gemessenen [39] Verlauf der Wärmeleitfähigkeit beobachtet (siehe Abbildung 4.16). Von dünnen Doppellagen aus kommend messen wir bei $\Lambda / c=1$ zunächst in etwa die Wärmeleitfähigkeit, wie wir sie auch in stöchiometrisch gleichem LSMO beobachten. Zu größeren Doppellagen sehen wir dann einen Abfall der Wärmeleitfähigkeit und ein Minimum zwischen $\Lambda / c=6$ und 8. Die Verschiebung des Minimums in Abhängigkeit der Temperatur, wie sie Ravichandran et al. gesehen haben [39], konnten wir nicht beobachten. Dies lässt sich allerdings einfach durch die anomale Temperaturabhängigkeit des LMO [109] bzw. der LMO/SMO-ÜG (siehe Kapitel 4.2.2) erklären. Bei noch größeren Doppellagen $\Lambda / c=10$ und $\Lambda / c=12$ sehen wir, dass sich die Wärmeleitfähigkeit wieder der Beschreibung durch den Kapitzawiderstand [124, 127] annähert (ver- 
gleiche Abb. 4.16 mit 4.11). Für noch größere Übergitter liegt das LMO wieder in der rhomboedrischen Struktur vor [80] und ein Vergleich der Wärmeleitfähigkeit zu den anderen ÜG ist nicht mehr sinnvoll.

Durch das Anpassen des Born-von-Kármán-Modells an unsere Messungen habe ich dann versucht, den Verlauf der gemessenen Wärmeleitfähigkeit anhand der mittleren freien Weglänge der Phononen und der phononischen Bandstruktur zu beschreiben. Das Bild des phononischen Wärmetransportes, welches sich hieraus ergibt, möchte ich in den nächsten Sätzen kurz zusammenfassen möchte:

Die phononische Wärmeleitfähigkeit $\kappa_{\mathrm{V}}$ eines Festkörpers kann über seine PhononenBandstruktur berechnet werden (siehe Gleichung 2.27). Bringt man zwei unterschiedliche Festkörper aneinander, entsteht an der Grenzfläche eine Fehlanpassung der Gitterparameter [123, 128] und der Energietransport durch Phononen erfährt einen zusätzlichen Widerstand. Dies konnten wir in ähnlicher Weise im GHz-Spektrum an den $\mathrm{MgO} / \mathrm{ZrO}_{2}$-Mulitlagen beobachten (siehe Kapitel 2.1 und 4.1.1). Dass diese Fehlanpassung nicht nur in makroskopischen Größen durch die elastischen Parameter wie Dichte und Schallgeschwindigkeit definiert werden kann, sondern eine Folge der Fehlanpassung der Bandstrukturen ${ }^{1}$ ist, lässt sich anschaulich bei einem Vergleich der $m / n=1$ und $m / n=2$ Wärmeleitfähigkeiten (Abb. 4.11) mit der jeweiligen longitudinalen Dispersion in Abb. 2.14 sehen. Der Wärmetransport als Folge der phononischen Bandstruktur wird noch offensichtlicher, wenn wir uns die Messungen von $m / n=1$ (Abb. 4.16) anschauen. Die Betrachtung eigenständiger Bandstrukturen für LMO und SMO sind für Lagendicken von wenigen Atomen, bzw. von Schichtdicken unterhalb der freien Weglänge der Phononen, nicht mehr gerechtfertigt. In diesem Bild verlieren auch die materialspezifischen Wärmeleitfähigkeiten $\kappa_{\mathrm{LMO}}$ und $\kappa_{\mathrm{SMO}}$ und auch die Beschreibung des Übergitters als Reihenschaltung von Wärmewiderständen (Gleichung 4.4) ihre Bedeutung. Eine Bandstruktur des gesamten Übergitters definiert uns eine neue ÜG-Wärmeleitfähigkeit. So lässt sich schlussendlich auch der Anstieg der Wärmeleitfähigkeit für $\Lambda / c=2$ und 4 erklären: Größe und Anzahl der Bandlücken und die Gruppengeschwindigkeit $\partial \omega / \partial q$ verändern sich als Funktion von $\Lambda$ und nehmen so direkt Einfluss auf die phononische Wärmeleitfähigkeit des Übergitters.

\footnotetext{
${ }^{1}$ Diese steht natürlich in direktem Zusammenhang mit der Masse der Atome und Schallgeschwindigkeit.
} 
Auch wenn es sehr schwierig ist, allgemeine Aussagen anhand der komplizierten, dreidimensionalen Bandstruktur zu treffen, so sehen wir an der angepassten mittleren freien Weglänge der Phononen (Abb. 4.17 (a) und (b)), dass der Anstieg bei dünnen Übergittern mit einer Erhöhung der mittleren freien Weglänge aller beteiligten Phononen einhergeht. Mit Blick auf die Arbeit von Simkin und Mahan [32] und der Theorie aus Kapitel 2.2.2 können wir dies so verstehen, dass durch das Falten und Verschieben der Bandstruktur die Bandlücken gerade in den Bereich von Phononen mit niedriger freien Weglänge geschoben werden. Phononen mit diesen Frequenzen nehmen nicht mehr am thermischen Transport teil und die MFP $\xi$ steigt ohne den Beitrag dieser Phononen an. In Analogie zu den Experimenten an $\mathrm{MgO} / \mathrm{ZrO}_{2}$ können wir das ÜG auch als Bragg-Reflektor für diese Phononenmoden verstehen.

Zusammengefasst interpretiere ich die Änderung von $\xi$ wie folgt: Der Wärmetransport setzt sich aus kohärenten Phononen $(\xi \gg \Lambda)$ und inkohärenten Phononen $(\xi \leq \Lambda)$ zusammen. Schließt die Bandstruktur eines Übergitters einen Anteil der inkohärenten Phononen vom Transport aus, nimmt der kohärente Charakter des Transports zu und die Wärmeleitfähigkeit steigt an. Für große $\Lambda$ dominiert die intrinsische mittlere freie Weglänge der einzelnen Materialien im Übergitter und der Wärmetransport geht in das klassische inkohärente Modell (Gl. (2.24) und (4.4)) über.

Ergänzend möchte ich noch die $\lambda / c=4$ Probe mit antiferromagnetischer Kopplung (siehe magnetometrische Messungen in Abb. 4.19) erwähnen, welche durch leichte Variation der Probenstöchiometrie hergestellt wurde. Im Bereich zwischen $T_{\mathrm{FM}}$ und $T_{\mathrm{AFM}}$ beobachten wir im direkten Vergleich mit einer Probe ohne AFM-Kopplung ein Absinken der Wärmeleitfähigkeit um fast 50\%. Durch Anpassen unseres Modells an die Messdaten äußert sich dieser Abfall in einer ähnlich großen Abnahme der mittleren freien Weglänge bzw. des Kohärenzgrades der Phononen. Dies können wir so interpretieren, als dass AFM-Magnonenmoden mit Phononenmoden hybridisieren und neue Magnon-Polaronen-Moden entstehen [116]. Als neuer Streukanal für die Phononen reduziert dies $\xi$ und damit auch den Wärmetransport in unserem Übergitter. Im Bild des kohärenten Wärmetransports können wir dies auch als das Öffnen neuer Bandlücken im Spektrum der Phononen [119, 120] verstehen. Die neuen Bandlücken blockieren einen Teil der Phononenmoden. Da diese Bandlücken 
in unserem BK-Modell nicht berücksichtigt sind, führt Anpassen des Modells an die Messdaten zu scheinbar kleineren freien Weglängen $\xi$ der Phononen.

Diese Interpretationen der Messergebnisse folgt hauptsächlich aus dem Vergleich von experimentell bestimmten Wärmeleitfähigkeiten und den kritischen Temperaturen der magnetischen Phasen. Einen direkten Nachweis des Einflusses der AFM-Phase auf die Wärmeleitfähigkeit bleibe ich an dieser Stelle leider schuldig und muss an zukünftige Experimente verweisen. Ein starker Hinweis auf die Wechselwirkung zwischen Magnonen und Phononen in diesen Übergittern wäre beispielsweise ein Anstieg der Wärmeleitfähigkeit wenn ein ausreichend starkes äußeres Magnetfeld die AFM-Phase aufhebt. In einfachen Versuchen mit NeodymMagneten im TTR-Probenhalter konnte keine Änderung der Wärmeleitfähigkeit gemessen werden, jedoch liegt es nahe, dass die Feldstärke eines Neodymmagneten nicht ausreicht, um die kritische Feldstärke der AFM-Phase aufzubrechen [121]. Eine Erweiterung des TTR-Experimentes mit Magnetspulen war aufgrund der Pandemie zeitlich nicht mehr möglich. Eine weitere Möglichkeit wären auch magnetfeldabhängige 3w-Messungen in einem PPMS-System. Ein anderer Ansatz, die hier präsentierten Ergebnisse und Schlussfolgerungen zu überprüfen, wäre eine Validierung der von uns mit dem BK-Modell berechneten Phononenspektren. Dies könnte zum Beispiel mit Hilfe von DFT-Berechnungen [79, 129, 130] erfolgen. Experimentell könnte das Spektrum der Phononen mit inelastischer Neutronenstreuung [77, 78] bestimmt werden. Die niederfrequenten Zweige der Phononendispersion und Magnonenmoden in diesem Frequenzbereich könnten mit BrillouinLichtstreuung [131] experimentell untersucht werden.

Sollten diese Untersuchungen positiv ausfallen, könnte der Wärmetransport in LMO/SMO-ÜG mit AFM-Kopplung auch dahin funktionalisiert werden, dass mit einem externen Feld die AFM-Phase unterdrückt und damit die Wärmeleitfähigkeit zwischen verschiedenen Werten hin und her geschaltet werden kann. Solch ein Wärmeschalter eröffnet spannende Anwendungsszenarien, z.B. zum Wärmemanagment von Mikrochips oder für umschaltbare Wärmedämmschichten. 


\subsubsection{Ausblick: Phononenisolator}

Als Ausblick möchte ich noch ein Übergitter vorstellen, welches wir mit Hilfe unseres BK-Modells berechnet haben. Bis jetzt konnte dieses Übergitter jedoch noch nicht experimentell untersucht werden.

Die Idee war es hier, alle Erkenntnisse aus den Messungen der LMO/SMO-ÜG und unseren Simulationen zu kombinieren und die phononische Bandstruktur im Hinblick auf eine minimale Wärmeleitfähigkeit zu optimieren. Die so berechnete Bandstruktur ist in Abbildung 5.2 dargestellt.

(a)

(b)

(c)

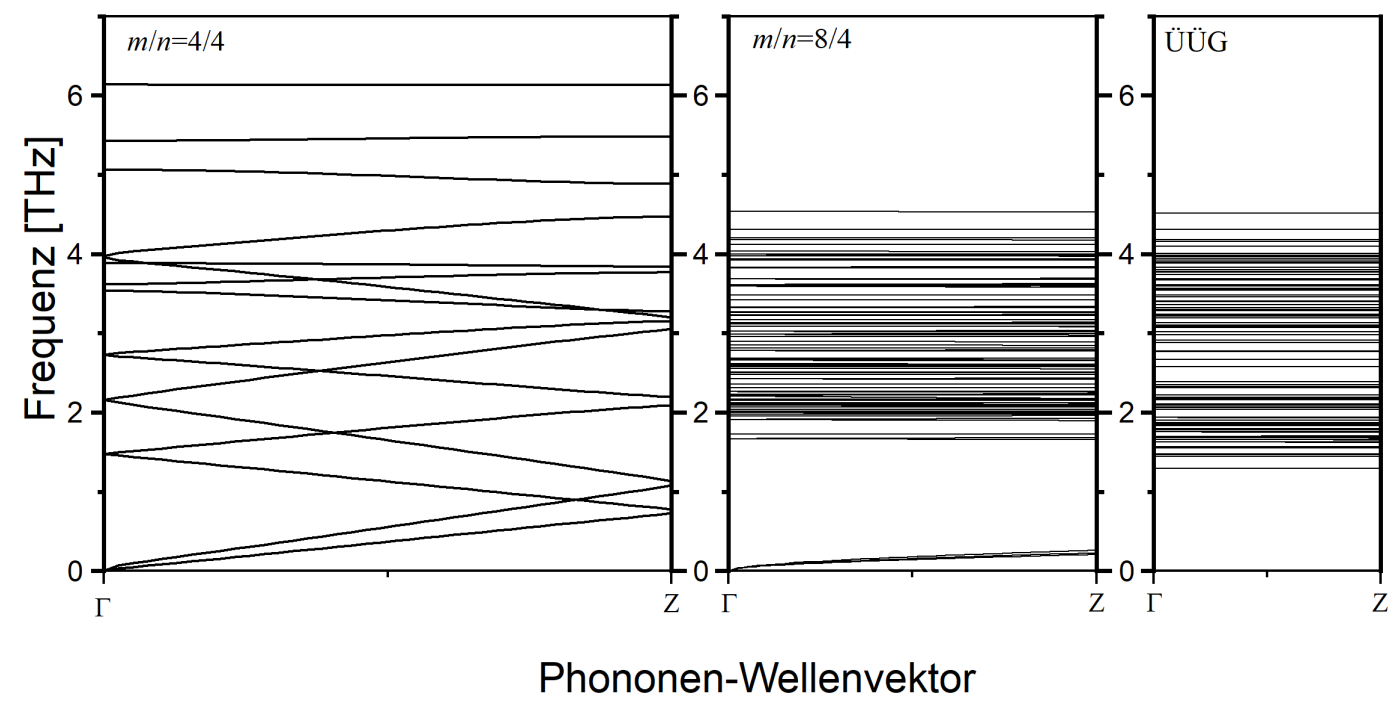

Abbildung 5.2: Bandstrukturen [80] von (a) $(\mathrm{LMO})_{4} /(\mathrm{SMO})_{4}$ berechnet mit kubischem Gitter, (b) $(\mathrm{LMO})_{8} /(\mathrm{SMO})_{4}$ mit rhomboedrischem LMO und (c) $(\mathrm{LMO})_{10} /(\mathrm{SMO})_{5} /(\mathrm{LMO})_{5} /(\mathrm{SMO})_{5}$ mit einer 10 Monolagen dicken Schicht rhomboedrischem LMO und einer 5 Monolagen dicken kubischen LMO-Schicht.

Die Bandstruktur in (c) ist das Resultat, wenn wir die Eigenschaften von $m / n=1$ (a) und $m / n=2$ (b) Übergittern miteinander vereinen. Das dargestellte Übergitter besteht aus einer $(\mathrm{LMO})_{10} /(\mathrm{SMO})_{5}$ Doppellage mit rhomboedrischem LMO, gefolgt von einer $(\mathrm{LMO})_{5} /(\mathrm{SMO})_{5}$ mit kubischem LMO. Aufgrund seiner Strukturierung nennen wir dieses $m / n / n / n=10 / 5 / 5 / 5$ Übergitter auch Überübergitter (ÜÜG). Wir sehen in dieser Bandstruktur, dass die akustischen Zwei- 
ge zu sehr niedrigen Frequenzen gedrückt werden und alle Bänder oberhalb der ersten Bandlücke sehr stark abflachen. Die Berechnung der Wärmeleitfähigkeit dieser Bandstruktur nach Gleichung 2.30 ergibt einen theoretischen Wert von $\kappa_{\mathrm{SÜG}}=0.01 \mathrm{~W} \mathrm{~m}^{-1} \mathrm{~K}^{-1}$. An dieser Stelle sei angemerkt, dass nach Berechnungen von Cohn et al. [109] die Wärmeleitfähigkeit von rhomboedrischem LMO mit $\kappa_{\mathrm{LMO}}=0.7 \mathrm{~W} \mathrm{~m}^{-1} \mathrm{~K}^{-1}$ und damit nur knapp über dem amorphen Limit $[132,133]$ der Wärmeleitfähigkeit bei Raumtemperatur von $\kappa_{\text {Limit }}=0.5 \mathrm{~W} \mathrm{~m}^{-1} \mathrm{~K}^{-1}[109,133]$ liegt. Die mittlere freie Weglänge der noch am thermischen Transport beteiligten Phononenmoden berechnen wir mit Hilfe des Callaway-Modells[110] und den an unsere Messdaten angepassten Parametern für Phonon-Phonon- und RayleighStreuung und erhalten für das ÜÜG einen Wert von $\xi=11.41 c$. Damit liegt in dieser Struktur die MFP etwa bei der Hälfte von $\Lambda / c=25$. Mit der berechneten Wärmeleitfähigkeit des ÜÜG unterschreiten wir das amorphe Limit der Wärmeleitfähigkeit. Für die mittlere freie Weglänge $\xi$ gilt im amorphen Limit eigentlich $\xi=1 \cdot c$ [134]. In unserem ÜÜG unterschreiten wir die Wärmeleitfähigkeit im amorphen Limit allerdings dadurch, dass wir die Phononen im Inneren unserer Struktur einschließen.

Eine experimentelle Realisierung dieser Proben steht zum jetzigen Zeitpunkt aufgrund von im Vergleich zu den anderen Übergittern etwas herausfordernder Wachstumsparameter leider noch aus. Bei einer erfolgreichen Umsetzung dieses Konzeptes hätten wir ein System mit einer bis heute unerreicht niedrigen Wärmeleitfähigkeit gefunden, sogar unter der Wärmeleitfähigkeit von Luft $\left(0.026 \mathrm{~W} \mathrm{~m}^{-1} \mathrm{~K}^{-1}\right.$ [135]) und das in der Form einer Dünnschicht.

Ich hoffe, dass ich mit dieser Dissertation und den dazugehörigen Veröffentlichung [26], [80] und [136] einen Beitrag zum Verständnis des phononischen Wärmetransportes in Nanostrukturen beitragen konnte. 



\section{Danksagung}

In diesen nächsten Zeilen möchte ich allen Personen danken, ohne deren Hilfe die vorliegende Dissertation niemals in dieser Form fertig geworden wäre.

An erster Stelle möchte ich meinen Eltern, Sabine und Klaus, danken. Ohne ihre moralische und finanzielle Unterstützung hätte ich mein Studium niemals durchführen und damit auch diese Dissertation nicht schreiben können.

Anschließend möchte ich meinem Betreuer Dr. Henning Ulrichs zuallererst dafür danken, dass er mir dieses Projekt angeboten hat. Ich danke ihm auch für seine herausragende Betreuung, Beratung und seine Hilfe im Labor. Ein besonderer Dank gilt ihm auch noch einmal dafür, dass er mir die Möglichkeit geboten hat an seinem VR-Labor-Projekt mitzuwirken.

Ich danke Prof. Dr. Vasily Moshnyaga für die Betreuung meiner Promotion und für die Hilfe bei Papern und meinen Fragen zu Manganaten und der MAD.

Ich danke Prof. Dr. Christan Jooss dafür, dass er sich als Zweitgutachter für meine Dissertation zu Verfügung gestellt hat und außerdem danke ich ihm dafür, dass er uns während des TTR-Aufbaus seinen ns-Laser zur Verfügung gestellt hat.

Ich danke Dr. Daniel Steil für viele lehrreiche Diskussionen und Hilfe bei konkreten Problemen mit Optik und Lasern.

Ich danke allen Mitgliedern meiner Prüfungskommission, dass sie sich bereit erklärt haben, an meiner Disputation teilzunehmen. Dies sind in keiner besonderen Reihenfolge: Dr. Henning Ulrichs, Prof. Dr. Christian Jooss, Prof. Dr. Vasily Moshnyaga, Prof. Dr. Matthias Krüger, Dr. Daniel Steil und Dr. Oliver Bünermann.

Ich danke dem gesamten I. Physikalischen Institut für die tolle Zeit, interessante Vorträge und eine angenehme Atmosphäre. Ein besonderer Dank gilt auch dem 
technischen Personal, ganz speziell Kai Dörner, Carsten Mahn, Bastian Beyland und Hartmut Eichenberg, für ihre technische Unterstützung bei meinen vielfältigen Aufbauten und Experimenten.

Ich danke der kompletten AG Ulrichs und AG Moshnyaga für die tolle Zusammenarbeit. Besonders danken möchte ich meinen Masteranten Daniel Metternich, Christopher Heins, Sina Ludewig, Tobias Claus und Roland Potthast, sowie meinem Bürokollegen Vitaly Bruchmann-Bamberg.

Ich danke der AG Münzenberg und dabei ganz besonders Prof. Dr. Markus Münzenberg und Dr. Jakob Walowski für die Möglichkeit in ihren Laboren in Greifswald Proben herzustellen und Messungen durchzuführen.

Ich danke allen Mitgliedern des Sonderforschungsbereich 1073 für den tollen Rahmen den der SFB meiner Promotion zur Verfügung gestellt hat. Speziell möchte ich dabei Prof. Dr. Christian Jooss, Dr. Jörg Hoffmann, Carmen Kaspar und Regina Vinnen für ihre organisatorischen Beiträge zum SFB danken.

Ich danke Maike Dawidowski, Uta Schwarzer, Christopher Heins und Vitaly Bruchmann-Bamberg für das Korrekturlesen meiner Dissertation.

$\mathrm{Zu}$ guter Letzt danke ich der Deutschen Forschungsgemeinschaft und dem SFB 1073 für die Finanzierung meines Projektes. 


\section{Abgrenzung}

Wissenschaftliche Forschung ist eine Gruppenaufgabe und so waren auch an der Erhebung und Auswertung der Daten dieser Arbeit neben mir selbst weitere Leute beteiligt. Zwischen den Beiträgen einzelner Personen oder Gruppen möchte ich in diesem Kapitel differenzieren.

Die $\mathrm{MgO} / \mathrm{ZrO}_{2}$ Multilagen wurden von Florian Döring und der Gruppe um HansUlrich Krebs im Institut für Materialphysik der Universität Göttingen hergestellt und mit Röntgenreflektometrie charakterisiert.

Die PPR-Messungen an $\mathrm{MgO} / \mathrm{ZrO}_{2}$ Multilagen wurden von Henning Ulrichs, Markus Müller und mir an einem Aufbau der AG Münzenberg der Universität Greifswald durchgeführt. Jakob Walowski hat bei der Justage des Aufbaus untersützt.

Die FDTD-Simulation wurde unter Anleitung von Henning Ulrichs von mir im Rahmen meiner Masterarbeit programmiert. Das zugehörige Auswertungsprogramm wurde von Markus Müller programmiert. Seitdem wurde das Programm von Henning Ulrichs, Daniel Metternich und mir verbessert und erweitert. Die FDTDSimulationen in dieser Arbeit wurden von mir durchgeführt und analysiert.

Die LMO/SMO Übergitter wurden von der Arbeitsgruppe von Prof. Dr. Moshnyaga hergestellt und mit XRD, XRR, AFM und SQUID charakterisiert. Speziell wurde die $m / n=2$ und die erste $m / n=1$-Probenserie von Marius Keunecke und Jan Philipp Bange an der MAD hergestellt. Die zweite $m / n=1$-Probenreihe wurde von Pia Henning hergestellt und charakterisiert. LMO und LSMO Proben wurden von Vitaly Bruchmann-Bamberg hergestellt.

Die UHV-Anlage zum Aufdampfen der TTR-Kupferschichten wurde von mir mit Unterstützung durch die Studenten Lisa Hanke und Daniel Metternich, sowie unseren Physiklaboranten Kai Dörner aufgebaut.

Der Transiente Thermoreflektivitätsaufbau wurde von mir zusammen mit den Masteranten Roland Potthast und Daniel Metternich entwickelt. Der TTR-Probenhalter 
wurde von mir in Autodesk Inventor mit technischer Unterstützung durch Kai Dörner konstruiert und von der feinmechanischen Werkstatt des I. Physikalischen Instituts umgesetzt.

Die TTR-Messungen an $m / n=2$ und $(\mathrm{LMO})_{10} /(\mathrm{SMO})_{10}$ wurden von Daniel Metternich und Roland Potthast gemessen. Alle weiteren TTR-Messungen wurden von mir durchgeführt.

TEM-Bilder und Bindungswinkelanalyse von LMO/SMO Übergittern stammen von Robert Gruhl, Dr. Ulrich Roß und Dr. Vladimir Roddatis. DFT-Rechnungen wurden von Dr. Igor Maznichenko und Dr. Sergey Ostanin der Universität Halle durchgeführt.

Das TTR-Auswertungsprogramm wurde von mir geschrieben. Die verwendeten Algorithmen basieren teilweise auf früheren Implementationen von Daniel Metternich und einer Anleitung von Henning Ulrichs.

Die Born-von-Kármán-Simulation wurde von mir geschrieben. Sie basiert auf einem Scilab-Script von Henning Ulrichs. Die Modelle für ÜÜG, Bulk-LMO, Bulk-SMO wurden von Henning Ulrichs implementiert. Die Analyse der Simulationsdaten wurde von mir und Henning Ulrichs durchgeführt.

MuMax3-Simulationen wurden von Henning Ulrichs und mir durchgeführt. 


\section{Abkürzungsverzeichnis}

TTR transiente Thermoreflektivitiät

TTTR temperaturabhängige transiente Thermoreflektivitiät

PPR Pump-Probe-Reflektivität

ÜG Übergitter

ÜÜG Überübergitter

ML Multilage

LMO $\mathrm{LaMnO}_{3}$

SMO $\quad \mathrm{SrMnO}_{3}$

LSMO $\mathrm{La}_{x} \mathrm{Sr}_{1-x} \mathrm{MnO}_{3}$

BTE Boltzmann-Transport-Gleichung

TDTR zeitabhängige Thermoreflektivität

MAD Metallorgansiche Aerosoldeposition

UHV Ultrahochvakuum

EBPVD Elektronenstrahlverdampfen

BK Born-von-Kármán

BKM Born-von-Kármán-Modell

DFT Dichtefunktionaltheorie

PLD Pulsed-Laser-Deposition 
FDTD Finite-differences time-domain

CW Continuous wave

SFB Sonderforschungsbereich 1073

FM ferromagnetisch

AFM antiferromagnetisch

PPMS Physical Property Measurement System

SQUID Superconducting Quantum Interference Device

MFP mittlere freie Weglänge 


\section{Literaturverzeichnis}

[1] Thomson, William: XV.- On the Dynamical Theory of Heat, with numerical results deduced from Mr Joule's Equivalent of a Thermal Unit, and M. Regnault's Observations on Steam. In: Transactions of the Royal Society of Edinburgh 20 (1853), Nr. 2, S. 261-288. http://dx.doi.org/10.1017/ S0080456800033172. - DOI 10.1017/S0080456800033172. - ISSN 0080-4568

[2] Schroeder, M. R.: Binaural dissimilarity and optimum ceilings for concert halls: More lateral sound diffusion. In: The Journal of the Acoustical Society of America 65 (1979), Nr. 4, 958-963. http://dx.doi.org/10.1121/1. 382601. - DOI 10.1121/1.382601. - ISSN 0001-4966

[3] Zhu, Yifan ; Fan, Xudong ; Liang, Bin ; Cheng, Jianchun ; Jing, Yun: Ultrathin Acoustic Metasurface-Based Schroeder Diffuser. In: Physical Review X 7 (2017), Nr. 2, S. 021034. http://dx.doi.org/10.1103/PhysRevX.7. 021034. - DOI 10.1103/PhysRevX.7.021034

[4] MusicProducer: Datei:Skylinediffusor EPS Material.jpg - Wikipedia. https://de.wikipedia.org/wiki/Datei:Skylinediffusor_EPS_ Material.jpg. Version: 24.06.2021

[5] GarczyK, Żaneta ; Stach, Sebastian ; ŢĂLu, Ştefan ; Sobola, Dinara ; WróBEL, Zygmunt: Stereometric Parameters of Butterfly Wings. In: Journal of Biomimetics, Biomaterials and Biomedical Engineering 31 (2017), S. 1-10. http://dx.doi .org/10.4028/www. scientific.net/JBBBE.31.1. - DOI 10.4028/www.scientific.net/JBBBE.31.1

[6] LiU, Kesong ; JiAng, Lei: Multifunctional integration: from biological to bio-inspired materials. In: ACS nano 5 (2011), Nr. 9, S. 6786-6790. http: //dx.doi.org/10.1021/nn203250y. - DOI 10.1021/nn203250y 
[7] Narasimhan, Vinayak ; Siddique, Radwanul H. ; Lee, Jeong O. ; Kumar, Shailabh ; Ndjamen, Blaise ; Du, Juan ; Hong, Natalie ; SretaVAN, David ; CHOO, Hyuck: Multifunctional biophotonic nanostructures inspired by the longtail glasswing butterfly for medical devices. In: Nature Nanotechnology 13 (2018), Nr. 6, 512-519. http://dx.doi.org/10.1038/ s41565-018-0111-5. - DOI 10.1038/s41565-018-0111-5. - ISSN 1748-3395

[8] Siddique, Radwanul H. ; Gomard, Guillaume ; Hölscher, Hendrik: The role of random nanostructures for the omnidirectional anti-reflection properties of the glasswing butterfly. In: Nature Communications 6 (2015), Nr. 1, 6909. http://dx.doi.org/10.1038/ncomms7909. - DOI 10.1038/ncomms7909. - ISSN 2041-1723

[9] Zinth, Wolfgang ; Aumüller, Ursula: Optik: Lichtstrahlen - Wellen Photonen. 4., aktualisierte Aufl. München : Oldenbourg, 2013. http: //dx.doi.org/10.1524/9783486735819. http://dx.doi.org/10.1524/ 9783486735819. - ISBN 9783486735819

[10] Luckyanova, M. N. ; Mendoza, J. ; Lu, H. ; Song, B. ; Huang, S. ; Zhou, J. ; Li, M. ; Dong, Y. ; Zhou, H. ; Garlow, J. ; Wu, L. ; Kirby, B. J. ; Grutter, A. J. ; Puretzky, A. A. ; Zhu, Y. ; Dresselhaus, M. S. ; Gossard, A. ; Chen, G.: Phonon localization in heat conduction. In: Science Advances 4 (2018), Nr. 12, eaat9460. http://dx.doi.org/10. 1126/sciadv .aat9460. - DOI 10.1126/sciadv.aat9460. - ISSN 2375-2548

[11] Khvesyuk, V. I. ; Qiao, W. ; Barinov, A. A.: The effect of phonon diffusion on heat transfer. In: Journal of Physics: Conference Series 1385 (2019), Nr. 1, 012046. http://dx.doi.org/10.1088/1742-6596/1385/1/ 012046. - DOI 10.1088/1742-6596/1385/1/012046. - ISSN 1742-6596

[12] Hunklinger, Siegfried: Festkörperphysik. 3., verb. und aktualisierte Aufl. München : Oldenbourg, 2011 (Mathematik, Physik 10-2012). http: //dx.doi.org/10.1524/9783486711547. http://dx.doi.org/10.1524/ 9783486711547. - ISBN 9783486705478

[13] Narayanamurti, V. ; Dynes, R. C.: Observation of Second Sound in Bismuth. In: Physical Review Letters 28 (1972), Nr. 22, S. 1461-1465. http: 
//dx.doi.org/10.1103/PhysRevLett.28.1461. - DOI 10.1103/PhysRevLett.28.1461. - ISSN 0031-9007

[14] PoHL, Dieter W.: Light Scattering from Second Sound in NaF. Version: 1976. http://dx.doi.org/10.1007/978-1-4613-4271-7\{_\}27. In: Challis, L. J. (Hrsg.) ; Rampton, V. W. (Hrsg.) ; Wyatt, A. F. G. (Hrsg.): Phonon Scattering in Solids. Boston, MA : Springer, 1976. - DOI 10.1007/978-1-4613-4271-7_27. - ISBN 978-1-4613-4271-7, S. 102-104

[15] Mikhail, I. F. I. ; Simons, S.: The Propagation of High Frequency Thermal Waves in Dielectric Crystals. Version: 1976. http://dx.doi.org/10.1007/ 978-1-4613-4271-7\{_\}26. In: Challis, L. J. (Hrsg.) ; Rampton, V. W. (Hrsg.) ; Wyatt, A. F. G. (Hrsg.): Phonon Scattering in Solids. Boston, MA : Springer, 1976. - DOI 10.1007/978-1-4613-4271-7_26. - ISBN 9781-4613-4271-7, S. 99-101

[16] Clarke, David R. ; Oechsner, Matthias ; Padture, Nitin P.: Thermalbarrier coatings for more efficient gas-turbine engines. In: MRS Bulletin 37 (2012), Nr. 10, 891-898. http://dx.doi.org/10.1557/mrs.2012.232. - DOI 10.1557/mrs.2012.232. - ISSN 1938-1425

[17] Masrura, Haque M. ; Kareekunnan, Afsal ; Liu, Fayong ; Ramaraj, Sankar G. ; Ellrott, Günter ; Hammam, Ahmmed M. M. ; MuruganaThan, Manoharan ; Mizuta, Hiroshi: Design of Graphene Phononic Crystals for Heat Phonon Engineering. In: Micromachines 11 (2020), Nr. 7. http://dx.doi.org/10.3390/mi11070655. - DOI 10.3390/mi11070655. ISSN 2072-666X

[18] Hu, Ming ; YANG, Zhonghua: Perspective on multi-scale simulation of thermal transport in solids and interfaces. In: Physical Chemistry Chemical Physics 23 (2021), Nr. 3, 1785-1801. http://dx.doi.org/10.1039/ D0CP03372C. - DOI 10.1039/D0CP03372C. - ISSN 1463-9084

[19] J. G. Koller ; W. C. Athas ; L. J. Svensson: Thermal logic circuits. In: Proceedings Workshop on Physics and Computation. PhysComp '94, 1994, S. 119-127 
[20] Paolucci, Federico ; Marchegiani, Giampiero ; Strambini, Elia ; GiazotTo, Francesco: Phase-Tunable Thermal Logic: Computation with Heat. In: Physical Review Applied 10 (2018), Nr. 2, S. 024003. http://dx.doi. org/10.1103/PhysRevApplied.10.024003. - DOI 10.1103/PhysRevApplied.10.024003

[21] Vahala, K. ; Herrmann, M. ; Knünz, S. ; Batteiger, V. ; SaAthoff, G. ; HÄnsch, T. W. ; Udem, Th.: A phonon laser. In: Nature Physics 5 (2009), Nr. 9, 682-686. http://dx.doi.org/10.1038/nphys1367. - DOI 10.1038/nphys1367. - ISSN 1745-2481

[22] Anufriev, Roman ; Maire, Jeremie ; Nomura, Masahiro: Review of coherent phonon and heat transport control in one-dimensional phononic crystals at nanoscale. In: APL Materials 9 (2021), Nr. 7, 070701. http: //dx.doi.org/10.1063/5.0052230. - DOI 10.1063/5.0052230

[23] Khelif, A. ; Choujaa, A. ; Benchabane, S. ; Duafari-Rouhani, B. ; LAUDE, V.: Guiding and bending of acoustic waves in highly confined phononic crystal waveguides. In: Applied Physics Letters 84 (2004), Nr. 22, 44004402. http://dx.doi.org/10.1063/1.1757642. - DOI 10.1063/1.1757642. - ISSN 0003-6951

[24] Staff, Science X.: Amplifier for terahertz lattice vibrations in a semiconductor crystal. In: Phys.org (04.04.2019). https://phys.org/news/ 2019-04-amplifier-terahertz-lattice-vibrations-semiconductor . html

[25] Jäger, J. V. ; Scherbakov, A. V. ; Glavin, B. A. ; Salasyuk, A. S. ; Campion, R. P. ; Rushforth, A. W. ; Yakovlev, D. R. ; Akimov, A. V. ; BAYER, M.: Resonant driving of magnetization precession in a ferromagnetic layer by coherent monochromatic phonons. In: Physical Review B 92 (2015), Nr. 2, S. 020404. http://dx.doi.org/10.1103/PhysRevB.92.020404. DOI 10.1103/PhysRevB.92.020404

[26] Ulrichs, Henning ; Meyer, Dennis ; Döring, Florian ; Eberl, Christian ; KREBS, Hans-Ulrich: Spectral control of elastic dynamics in metallic nanocavities. In: Scientific Reports 7 (2017), Nr. 1, 10600. http://dx.doi. 
org/10.1038/s41598-017-11099-y. - DOI 10.1038/s41598-017-11099-y.

- ISSN 2045-2322

[27] Fourier, Jean-Baptiste-Joseph: The analytical theory of heat. Cambridge and Cambridge : At the University Press Printed by C.J. Clay M.A. at the University Press, 1878 http://worldcatlibraries.org/wcpa/oclc/ 1178829

[28] Joule, J. P.: On the changes of temperature produced by the rarefaction and condensation of air. In: The London, Edinburgh, and Dublin Philosophical Magazine and Journal of Science 26 (1845), Nr. 174, S. 369-383. http://dx.doi.org/10.1080/14786444508645153. - DOI 10.1080/14786444508645153. - ISSN 1941-5966

[29] Mayer, J. R.: Bemerkungen über die Kräfte der unbelebten Natur. In: $A n$ nalen der Chemie und Pharmacie 42 (1842), Nr. 2, S. 233-240. http://dx. doi.org/10.1002/jlac.18420420212. - DOI 10.1002/jlac.18420420212. ISSN 00754617

[30] Frenkel, J.: International Series of Monographs on Physics. Bd. ed. by R.H. Fowler and P. Kapitza: Wave Mechanics: Elementary Theory. Clarendon Press, 1932 https://books.google.de/books?id=XqvIugEACAAJ

[31] Ziman, John M.: Electrons and Phonons: The Theory of Transport Phenomena in Solids: The theory of transport phenomena in solids. Oxford : Oxford Press and Clarendon Press, 2001 (Oxford classic texts in the physical sciences). https://oxford.universitypressscholarship.com/view/10. 1093/acprof : oso/9780198507796.001.0001/acprof-9780198507796.

ISBN 0198507798

[32] Simkin ; Mahan: Minimum thermal conductivity of superlattices. In: Physical Review Letters 84 (2000), Nr. 5, S. 927-930. http://dx.doi.org/10. 1103/PhysRevLett.84.927. - DOI 10.1103/PhysRevLett.84.927. - ISSN 0031-9007 
[33] Holland, M. G.: Analysis of Lattice Thermal Conductivity. In: Physical Review 132 (1963), Nr. 6, S. 2461-2471. http://dx.doi.org/10.1103/ PhysRev.132.2461. - DOI 10.1103/PhysRev.132.2461

[34] Asheghi, M. ; Touzelbaev, M. N. ; Goodson, K. E. ; Leung, Y. K. ; Wong, S. S.: Temperature-Dependent Thermal Conductivity of SingleCrystal Silicon Layers in SOI Substrates. In: Journal of Heat Transfer 120 (1998), Nr. 1, S. 30-36. http://dx.doi.org/10.1115/1.2830059. - DOI 10.1115/1.2830059. - ISSN 0022-1481

[35] Uma, S. ; McConnell, A. D. ; Asheghi, M. ; Kurabayashi, K. ; GoodSON, K. E.: Temperature-Dependent Thermal Conductivity of Undoped Polycrystalline Silicon Layers. In: International Journal of Thermophysics 22 (2001), Nr. 2, 605-616. http://dx.doi.org/10.1023/A:1010791302387. DOI 10.1023/A:1010791302387. - ISSN 1572-9567

[36] Cahill, David G. ; Ford, Wayne K. ; Goodson, Kenneth E. ; Mahan, Gerald D. ; Majumdar, Arun ; Maris, Humphrey J. ; Merlin, Roberto ; Phillpot, Simon R.: Nanoscale thermal transport. In: Journal of Applied Physics 93 (2003), Nr. 2, S. 793-818. http://dx.doi.org/10.1063/1. 1524305. - DOI 10.1063/1.1524305. - ISSN 0021-8979

[37] Sverdrup, P. G. ; Sinha, S. ; Asheghi, M. ; Uma, S. ; Goodson, K. E.: Measurement of ballistic phonon conduction near hotspots in silicon. In: Applied Physics Letters 78 (2001), Nr. 21, 3331-3333. http://dx.doi.org/ 10.1063/1.1371536. - DOI 10.1063/1.1371536. - ISSN 0003-6951

[38] Sverdrup, Per G. ; Sungtaek Ju, Y. ; Goodson, Kenneth E.: SubContinuum Simulations of Heat Conduction in Silicon-on-Insulator Transistors. In: Journal of Heat Transfer 123 (2001), Nr. 1, S. 130-137. http: //dx.doi.org/10.1115/1.1337651. - DOI 10.1115/1.1337651. - ISSN 0022-1481

[39] Ravichandran, Jayakanth; Yadav, Ajay K. ; Cheaito, Ramez ; Rossen, Pim B. ; Soukiassian, Arsen ; Suresha, S. J. ; Duda, John C. ; Foley, Brian M. ; Lee, Che-Hui ; Zhu, Ye ; Lichtenberger, Arthur W. ; Moore, 
Joel E. ; Muller, David A. ; Schlom, Darrell G. ; Hopkins, Patrick E. ; Majumdar, Arun ; Ramesh, Ramamoorthy ; Zurbuchen, Mark A.: Crossover from incoherent to coherent phonon scattering in epitaxial oxide superlattices. In: Nature materials 13 (2014), Nr. 2, S. 168-172. http: //dx.doi.org/10.1038/nmat3826. - DOI 10.1038/nmat3826. - ISSN 14761122

[40] Nomura, Masahiro ; Shiomi, Junichiro ; Shiga, Takuma ; Anufriev, Roman: Thermal phonon engineering by tailored nanostructures. In: $J a$ panese Journal of Applied Physics 57 (2018), Nr. 8, S. 080101. http: //dx.doi.org/10.7567/JJAP.57.080101. - DOI 10.7567/JJAP.57.080101. - ISSN 0021-4922

[41] Lu, Ming-Hui ; Feng, Liang ; Chen, Yan-Feng: Phononic crystals and acoustic metamaterials. In: Materials Today 12 (2009), Nr. 12, 34-42. http: //dx.doi.org/10.1016/S1369-7021(09)70315-3. - DOI 10.1016/S13697021(09)70315-3. - ISSN 1369-7021

[42] Ward, A. ; Broido, D. A.: Intrinsic phonon relaxation times from firstprinciples studies of the thermal conductivities of Si and Ge. In: Physical Review B 81 (2010), Nr. 8, S. 085205. http://dx.doi.org/10.1103/ PhysRevB.81.085205. - DOI 10.1103/PhysRevB.81.085205

[43] Maris, Humphrey J. ; TAmura, Shin-ichiro: Heat flow in nanostructures in the Casimir regime. In: Physical Review B 85 (2012), Nr. 5, S. 054304. http://dx.doi.org/10.1103/PhysRevB.85.054304.- DOI 10.1103/PhysRevB.85.054304

[44] Cahill, David G. ; Braun, Paul V. ; Chen, Gang ; Clarke, David R. ; Fan, Shanhui ; Goodson, Kenneth E. ; Keblinski, Pawel ; King, William P. ; Mahan, Gerald D. ; Majumdar, Arun ; Maris, Humphrey J. ; Phillpot, Simon R. ; Pop, Eric ; ShI, Li: Nanoscale thermal transport. II. 2003-2012. In: Applied Physics Reviews 1 (2014), Nr. 1, 011305. http://dx.doi.org/10.1063/1.4832615. - DOI 10.1063/1.4832615

[45] Minnich, A. J.: Advances in the measurement and computation of thermal phonon transport properties. In: Journal of physics. Condensed matter : an 
Institute of Physics journal 27 (2015), Nr. 5, 053202. http://dx.doi.org/ 10.1088/0953-8984/27/5/053202. - DOI 10.1088/0953-8984/27/5/053202

[46] Käding, O. W. ; Skurk, H. ; Goodson, K. E.: Thermal conduction in metallized silicon-dioxide layers on silicon. In: Applied Physics Letters 65 (1994), Nr. 13, S. 1629-1631. http://dx.doi.org/10.1063/1.112933. DOI 10.1063/1.112933. - ISSN 0003-6951

[47] Bykov, V. P.: Spontaneous Emission in a Periodic Structure. In: Soviet Journal of Experimental and Theoretical Physics 35 (1972), S. 269. - ISSN 1063-7761

[48] John: Strong localization of photons in certain disordered dielectric superlattices. In: Physical Review Letters 58 (1987), Nr. 23, S. 2486-2489. http: //dx.doi.org/10.1103/PhysRevLett.58.2486. - DOI 10.1103/PhysRevLett.58.2486. - ISSN 0031-9007

[49] YABLONOvitch: Inhibited spontaneous emission in solid-state physics and electronics. In: Physical Review Letters 58 (1987), Nr. 20, S. 2059-2062. http://dx.doi.org/10.1103/PhysRevLett.58.2059. - DOI 10.1103/PhysRevLett.58.2059. - ISSN 0031-9007

[50] Zengerle, R.: Light Propagation in Singly and Doubly Periodic Planar Waveguides. In: Journal of Modern Optics 34 (1987), Nr. 12, S. 1589-1617. http://dx.doi.org/10.1080/09500348714551531. - DOI 10.1080/09500348714551531. - ISSN 0950-0340

[51] BABA, Toshihiko: Slow light in photonic crystals. In: Nature Photonics 2 (2008), Nr. 8, S. 465-473. http://dx.doi.org/10.1038/nphoton.2008. 146. - DOI 10.1038/nphoton.2008.146. - ISSN 1749-4885

[52] Joannopoulos, J. D. ; Villeneuve, Pierre R. ; Fan, Shanhui: Photonic crystals: putting a new twist on light. In: Nature 386 (1997), Nr. 6621, S. 143149. http://dx.doi.org/10.1038/386143a0. - DOI 10.1038/386143a0. ISSN 0028-0836

[53] Joannopoulos, John D. ; Johnson, Steven G. ; Meade, Robert D. ; Johnnopoulos, John D. D. ; Johnson, Steven G. G. ; Winn, Joshua 
N. N. ; Meade, Robert D. D. ; Winn, Joshua N. III. ; Winn, Joshua N.: Photonic Crystals: Molding the Flow of Light. 2nd ed. Princeton : Princeton University Press, 2011. http://dx.doi.org/10.2307/j.ctvcm4gz9. http: //dx.doi.org/10.2307/j.ctvcm4gz9. - ISBN 9781400828241

[54] Ren, Shang Y. ; Dow, John D.: Thermal conductivity of superlattices. In: Physical review. B, Condensed matter 25 (1982), Nr. 6, S. 3750-3755. http://dx.doi.org/10.1103/PhysRevB.25.3750. - DOI 10.1103/PhysRevB.25.3750. - ISSN 0163-1829

[55] CAHIll, David G.: HEAT TRANSPORT IN DIELECTRIC THIN FILMS AND AT SOLID-SOLID INTERFACES. In: Microscale Thermophysical Engineering 1 (1997), Nr. 2, S. 85-109. http://dx.doi.org/10.1080/ 108939597200296. - DOI 10.1080/108939597200296. - ISSN 1091-7640

[56] Hyldgandd, Per ; Mahan, G. D.: Phonon superlattice transport. In: Physical review. B, Condensed matter 56 (1997), Nr. 17, S. 10754-10757. http://dx.doi.org/10.1103/PhysRevB.56.10754. - DOI 10.1103/PhysRevB.56.10754. - ISSN 0163-1829

[57] Chen, G.: Thermal conductivity and ballistic-phonon transport in the crossplane direction of superlattices. In: Physical review. B, Condensed matter 57 (1998), Nr. 23, S. 14958-14973. http://dx.doi.org/10.1103/PhysRevB. 57.14958. - DOI 10.1103/PhysRevB.57.14958. - ISSN 0163-1829

[58] Zhang, Peng ; JiAng, Maohua ; Zhue, Renjiang ; Zhang, Dingke ; Song, Yanrong: Thermal conductivity of GaAs/AlAs distributed Bragg reflectors in semiconductor disk laser: comparison of molecular dynamics simulation and analytic methods. In: Applied Optics 56 (2017), Nr. 15, 4537-4542. http: //dx.doi.org/10.1364/A0.56.004537. - DOI 10.1364/AO.56.004537. ISSN 2155-3165

[59] Luckyanova, Maria N. ; Garg, Jivtesh ; Esfarjani, Keivan ; JandL, Adam ; Bulsara, Mayank T. ; Schmidt, Aaron J. ; Minnich, Austin J. ; Chen, Shuo ; Dresselhaus, Mildred S. ; Ren, Zhifeng ; Fitzgerald, Eugene A. ; CHEn, Gang: Coherent phonon heat conduction in superlattices. 
In: Science 338 (2012), Nr. 6109, S. 936-939. http://dx.doi.org/10.1126/ science.1225549. - DOI 10.1126/science.1225549. - ISSN 1095-9203

[60] Li, Weixuan ; Chen, Xiang ; Zheng, Zexi ; Chen, Youping: Minimum thermal conductivity in periodically twinned SrTiO3. In: Computational Materials Science 112 (2016), 107-112. http://dx.doi.org/10.1016/j. commatsci.2015.10.020. - DOI 10.1016/j.commatsci.2015.10.020. - ISSN $0927-0256$

[61] HoŁuJ, Paulina ; Euler, Christoph ; Balke, Benjamin ; Kolb, Ute ; Fiedler, Gregor ; Müller, Mathis M. ; Jaeger, Tino ; Chávez Angel, Emigdio ; Kratzer, Peter ; JAKOB, Gerhard: Reduced thermal conductivity of TiNiSn/HfNiSn superlattices. In: Physical Review B 92 (2015), Nr. 12, S. 125436. http://dx.doi.org/10.1103/PhysRevB.92.125436. - DOI 10.1103/PhysRevB.92.125436

[62] Saha, Bivas ; Koh, Yee R. ; Feser, Joseph P. ; SAdasivam, Sridhar ; FisHer, Timothy S. ; Shakouri, Ali ; Sands, Timothy D.: Phonon wave effects in the thermal transport of epitaxial $\mathrm{TiN} /(\mathrm{Al}, \mathrm{Sc}) \mathrm{N}$ metal/semiconductor superlattices. In: Journal of Applied Physics 121 (2017), Nr. 1, 015109. http://dx.doi.org/10.1063/1.4973681. - DOI 10.1063/1.4973681. ISSN 0021-8979

[63] Ulrichs, Henning ; Meyer, Dennis ; Müller, Markus ; Wittrock, Steffen ; Mansurova, Maria ; Walowski, Jakob ; Münzenberg, Markus: $\mathrm{THz}$ elastic dynamics in finite-size CoFeB-MgO phononic superlattices. In: Journal of Applied Physics 120 (2016), Nr. 14, S. 142116. http: //dx.doi.org/10.1063/1.4961978. - DOI 10.1063/1.4961978. - ISSN 0021-8979

[64] Dennis Meyer: Elastische Dynamik in $\mathrm{CoFeB} / \mathrm{MgO}$ Übergittern. Göttingen, Georg-August-Universität Göttingen, Masterarbeit, April 2016

[65] Wang, Xinjiang ; Huang, Baoling: Computational study of in-plane phonon transport in Si thin films. In: Scientific Reports 4 (2014), Nr. 1, 6399. http://dx.doi.org/10.1038/srep06399. - DOI 10.1038/srep06399.

- ISSN 2045-2322 
[66] Jain, Ankit ; McGaughey, Alan J. H.: Thermal transport by phonons and electrons in aluminum, silver, and gold from first principles. In: Physical Review B 93 (2016), Nr. 8, S. 081206. http://dx.doi.org/10.1103/ PhysRevB.93.081206. - DOI 10.1103/PhysRevB.93.081206

[67] Johnson, P. B. ; Christy, R. W.: Optical Constants of the Noble Metals. In: Physical Review B 6 (1972), Nr. 12, S. 4370-4379. http://dx.doi.org/ 10.1103/PhysRevB.6.4370. - DOI 10.1103/PhysRevB.6.4370

[68] Matsuda, Osamu ; Larciprete, Maria C. ; Li Voti, Roberto ; Wright, Oliver B.: Fundamentals of picosecond laser ultrasonics. In: Ultrasonics 56 (2015), S. 3-20. http://dx.doi.org/10.1016/j.ultras. 2014.06.005. DOI 10.1016/j.ultras.2014.06.005

[69] Colvard, C. ; Merlin, R. ; Klein, M. V. ; Gossard, A. C.: Observation of Folded Acoustic Phonons in a Semiconductor Superlattice. In: Physical Review Letters 45 (1980), Nr. 4, S. 298-301. http://dx.doi.org/10.1103/ PhysRevLett.45.298. - DOI 10.1103/PhysRevLett.45.298. - ISSN 00319007

[70] C. Colvard ; T. A. Gant ; M. V. Klein ; R. Merlin ; R. Fischer ; H. Morkoc ; And A. C. Gossard: Folded acoustic and quantized optic phonons in (GaAl)As superlattices. (1985). http://dx.doi.org/10.1103/ PhysRevB.31.2080. - DOI 10.1103/PhysRevB.31.2080

[71] Ashcroft, Neil W. ; Mermin, N. D.: Festkörperphysik. 4., verb. Aufl. München : Oldenbourg, 2013. - ISBN 3486713019

[72] Ju, Y. S. ; Goodson, K. E.: Phonon scattering in silicon films with thickness of order 100 nm. In: Applied Physics Letters 74 (1999), Nr. 20, 3005-3007. http://dx.doi.org/10.1063/1.123994. - DOI 10.1063/1.123994. - ISSN 0003-6951

[73] Haynes, W. M.: CRC Handbook of Chemistry and Physics. CRC Press, 2016 https://books.google.de/books?id=VVezDAAAQBAJ. - ISBN 9781498754293 
[74] Song, Min-Kyoo ; Jhang, Kyung-Young: Crack Detection in SingleCrystalline Silicon Wafer Using Laser Generated Lamb Wave. In: Advances in Materials Science and Engineering 2013 (2013), S. 1-6. http: //dx.doi .org/10.1155/2013/950791. - DOI 10.1155/2013/950791. - ISSN 1687-8434

[75] Samsonov, G. V. (Hrsg.): Handbook of the Physicochemical Properties of the Elements. Boston, MA : Springer US, 1968. http://dx.doi.org/10.1007/ 978-1-4684-6066-7. http://dx.doi.org/10.1007/978-1-4684-6066-7. - ISBN 9781468460667

[76] Klemens, P. G.: Theory of lattice thermal conductivity: Role of low-frequency phonons. In: International Journal of Thermophysics 2 (1981), Nr. 1, 55-62. http://dx.doi.org/10.1007/BF00503574. - DOI 10.1007/BF00503574. - ISSN 1572-9567

[77] Dickens, M. H. ; Hutchings, M. T.: Inelastic neutron scattering study of the phonon dispersion relation of PbF 2 at 10K. In: Journal of Physics C: Solid State Physics 11 (1978), Nr. 3, 461-468. http://dx.doi.org/10. 1088/0022-3719/11/3/009. - DOI 10.1088/0022-3719/11/3/009. - ISSN $0022-3719$

[78] Klotz, S.: Phonon dispersion curves by inelastic neutron scattering to 12 Gpa. In: Zeitschrift für Kristallographie - Crystalline Materials 216 (2001), Nr. 8, S. 420-429. http://dx.doi.org/10.1524/zkri.216.8.420.20359. - DOI 10.1524/zkri.216.8.420.20359. - ISSN 2194-4946

[79] Fritsch, J.: Density functional calculation of semiconductor surface phonons. In: Physics Reports 309 (1999), Nr. 4-6, 209-331. http://dx.doi.org/ 10.1016/S0370-1573(98)00034-9. - DOI 10.1016/S0370-1573(98)000349. - ISSN 0370-1573

[80] Meyer, D. ; Roddatis, V. ; Bange, J. P. ; Lopatin, S. ; Keunecke, M. ; Metternich, D. ; Ross, U. ; Maznichenko, I. V. ; Ostanin, S. ; Mertig, I. ; Radisch, V. ; Egoavil, R. ; Lazić, I. ; Moshnyaga, V. ; ULRICHS, H.: Atomic scale spectral control of thermal transport in phononic crystal superlattices. https://arxiv.org/pdf/2009.14532 
[81] SøndenA, Rune ; Stølen, Svein ; Ravindran, P. ; Grande, Tor: Heat capacity and lattice dynamics of cubic and hexagonal SrMnO3 : Calorimetry and density functional theory simulations. In: Physical Review B 75 (2007), Nr. 21. http://dx.doi.org/10.1103/PhysRevB.75.214307. - DOI 10.1103/PhysRevB.75.214307

[82] Jain, Anubhav ; Ong, Shyue P. ; Hautier, Geoffroy ; Chen, Wei ; RIChards, William D. ; Dacek, Stephen ; Cholia, Shreyas ; Gunter, Dan ; Skinner, David ; Ceder, Gerbrand ; Persson, Kristin A.: Commentary: The Materials Project: A materials genome approach to accelerating materials innovation. In: APL Materials 1 (2013), Nr. 1, S. 011002. http://dx.doi.org/10.1063/1.4812323. - DOI 10.1063/1.4812323

[83] Yahia, Mondher ; Batis, Habib: Properties of Undoped and Ca-Doped LaMnO3 - Non-Stoichiometry and Defect Structure. In: European Journal of Inorganic Chemistry 2003 (2003), Nr. 13, 2486-2494. http://dx. doi.org/10.1002/ejic.200300025. - DOI 10.1002/ejic.200300025. - ISSN 14341948

[84] Ghosh, Barnali ; Siruguri, V. ; Raychaudhuri, A. K. ; ChatterJI, Tapan: Effect of size reduction on the structural and magnetic order in LaMnO nanocrystals: a neutron diffraction study. In: Journal of physics. Condensed matter : an Institute of Physics journal 26 (2014), Nr. 2, 025603. http://dx.doi.org/10.1088/0953-8984/26/2/025603. - DOI $10.1088 / 0953-8984 / 26 / 2 / 025603$

[85] Keunecke, Marius ; Lyzwa, Fryderyk ; Schwarzbach, Danny ; RodDatis, Vladimir ; Gauquelin, Nicolas ; Müller-Caspary, Knut ; VerBeeck, Johann ; Callori, Sara J. ; Klose, Frank ; Jungbauer, Markus ; Moshnyaga, Vasily: High- TC Interfacial Ferromagnetism in SrMnO 3 /LaMnO 3 Superlattices. In: Advanced Functional Materials 30 (2020), Nr. 18, S. 1808270. http://dx.doi.org/10.1002/adfm.201808270. - DOI 10.1002/adfm.201808270. - ISSN 1616-301X

[86] Döring, Florian ; Major, Anna ; Eberl, Christian ; Krebs, Hans-Ulrich: Minimized thermal conductivity in highly stable thermal barrier $\mathrm{W} / \mathrm{ZrO} 2$ 
multilayers. In: Applied Physics A 122 (2016), Nr. 10, 1-5. http://dx.doi. org/10.1007/s00339-016-0405-0. - DOI 10.1007/s00339-016-0405-0. ISSN 1432-0630

[87] Krebs, Hans-Ulrich ; Weisheit, Martin ; Faupel, Jörg ; Süske, Erik ; Scharf, Thorsten ; Fuhse, Christian ; Störmer, Michael ; Sturm, Kai ; Seibt, Michael ; KiJewski, Harald ; Nelke, Dorit ; PAnchenko, Elena ; Buback, Michael: Pulsed Laser Deposition (PLD) - A Versatile Thin Film Technique. Version:2003. http://dx.doi.org/10.1007/ 978-3-540-44838-9\{_\}36. In: Kramer, Bernhard (Hrsg.): Advances in Solid State Physics. Berlin and Heidelberg : Springer, 2003 (Advances in Solid State Physics). - DOI 10.1007/978-3-540-44838-9_36. - ISBN 9783-540-44838-9, S. 505-518

[88] Jungbauer, M. ; Hühn, S. ; Egoavil, R. ; Tan, H. ; Verbeeck, J. ; van Tendeloo, G. ; Moshnyaga, V.: Atomic layer epitaxy of RuddlesdenPopper $\mathrm{SrO}(\mathrm{SrTiO} 3$ ) n films by means of metalorganic aerosol deposition. In: Applied Physics Letters 105 (2014), Nr. 25, S. 251603. http://dx.doi. org/10.1063/1.4905055. - DOI 10.1063/1.4905055. - ISSN 0003-6951

[89] Ruello, Pascal ; Gusev, Vitalyi E.: Physical mechanisms of coherent acoustic phonons generation by ultrafast laser action. In: Ultrasonics 56 (2015), S. 21-35. http://dx.doi.org/10.1016/j.ultras.2014.06.004. - DOI $10.1016 /$ j.ultras.2014.06.004

[90] Akhmanov, S. A. ; Gusev, V. E.: Laser excitation of ultrashort acoustic pulses: New possibilities in solid-state spectroscopy, diagnostics of fast processes, and nonlinear acoustics. In: Soviet Physics Uspekhi 35 (1992), Nr. 3, 153-191. http://dx.doi.org/10 .1070/PU1992v035n03ABEH002221. - DOI 10.1070/PU1992v035n03ABEH002221. - ISSN 0038-5670

[91] Döring, Florian ; Ulrichs, Henning ; Pagel, Sinja ; MÜller, Markus ; Mansurova, Maria ; Müller, Matthias ; Eberl, Christian ; Erichsen, Torben ; Huebner, Dennis ; Vana, Philipp ; Mann, Klaus ; MÜnzenberG, Markus ; KREBS, Hans-Ulrich: Confinement of phonon propagation in laser deposited tungsten/polycarbonate multilayers. In: New Journal of Physics 
18 (2016), Nr. 9, 092002. http://dx.doi.org/10.1088/1367-2630/18/9/ 092002. - DOI 10.1088/1367-2630/18/9/092002. - ISSN 1367-2630

[92] CAhill ; POHL: Thermal conductivity of amorphous solids above the plateau. In: Physical review. B, Condensed matter 35 (1987), Nr. 8, S. 4067-4073. http://dx.doi.org/10.1103/PhysRevB.35.4067. - DOI 10.1103/PhysRevB.35.4067. - ISSN 0163-1829

[93] Cahill ; Katiyar ; Abelson: Thermal conductivity of a-Si:H thin films. In: Physical review. B, Condensed matter 50 (1994), Nr. 9, S. 6077-6081. http://dx.doi.org/10.1103/PhysRevB.50.6077. - DOI 10.1103/PhysRevB.50.6077. - ISSN 0163-1829

[94] Borca-Tasciuc, T. ; Kumar, A. R. ; Chen, G.: Data reduction in $3 \$ \backslash$ omega $\$$ method for thin-film thermal conductivity determination. In: Review of Scientific Instruments 72 (2001), Nr. 4, 2139-2147. http://dx. doi.org/10.1063/1.1353189. - DOI 10.1063/1.1353189. - ISSN 0034-6748

[95] Koh, Yee K. ; Singer, Suzanne L. ; Kim, Woochul ; Zide, Joshua M. O. ; Lu, Hong ; Cahill, David G. ; Majumdar, Arun ; Gossard, Arthur C.: Comparison of the $3 \$ \backslash$ omega $\$$ method and time-domain thermoreflectance for measurements of the cross-plane thermal conductivity of epitaxial semiconductors. In: Journal of Applied Physics 105 (2009), Nr. 5, 054303. http://dx.doi.org/10.1063/1.3078808. - DOI 10.1063/1.3078808. ISSN 0021-8979

[96] Guidotti, Daniel ; Wilman, John G.: Novel and nonintrusive optical thermometer. In: Applied Physics Letters 60 (1992), Nr. 5, 524-526. http://dx.doi.org/10.1063/1.107440. - DOI 10.1063/1.107440. - ISSN 0003-6951

[97] FlORIAN DÖRING: Minimizing thermal conductivity in laser deposited multilayers. Göttingen, Georg-August-Universität Göttingen, Dissertation, 2017. https://ediss.uni-goettingen.de/handle/11858/ 00-1735-0000-002B-7D24-B 
[98] Gengler, Jamie J. ; Roy, Sukesh ; Jones, John G. ; Gord, James R.: Two-color time-domain thermoreflectance of various metal transducers with an optical parametric oscillator. In: Measurement Science and Technology 23 (2012), Nr. 5, 055205. http://dx.doi.org/10.1088/0957-0233/23/5/ 055205. - DOI 10.1088/0957-0233/23/5/055205. - ISSN 0957-0233

[99] Ganeev, Rashid A. (Hrsg.): Nanostructured Nonlinear Optical Materials: Formation and Characterization. Amsterdam, Netherlands : Elsevier, 2018. - ISBN 978-0-12-814303-2

[100] Carslaw, Horatio S. ; Jaeger, John C.: Conduction of heat in solids. 2. Ed. Oxford : Clarendon Press, 1986 (Oxford science publications). - ISBN 0198533683

[101] Kamba, S. ; Goian, V. ; Skoromets, V. ; Hejtmánek, J. ; Bovtun, V. ; Kempa, M. ; Borodavka, F. ; Vaněk, P. ; Belik, A. A. ; Lee, J. H. ; PAcherová, O. ; Rabe, K. M.: Strong spin-phonon coupling in infrared and Raman spectra of SrMnO3. In: Physical Review B 89 (2014), Nr. 6, S. 064308. http://dx.doi.org/10.1103/PhysRevB.89.064308. DOI 10.1103/PhysRevB.89.064308

[102] Balageas, D. L. ; Krapez, J. C. ; Cielo, P.: Pulsed photothermal modeling of layered materials. In: Journal of Applied Physics 59 (1986), Nr. 2, 348357. http://dx.doi.org/10.1063/1.336690. - DOI 10.1063/1.336690.ISSN 0021-8979

[103] J. Nelder ; R. Mead: A Simplex Method for Function Minimization. In: undefined (1965). https://www.semanticscholar.org/ paper/A-Simplex-Method-for-Function-Minimization-Nelder-Mead/ 017ddb7e815236defd0566bc46f6ed8401cc6ba6

[104] AZoM.com: Properties: Zirconia - ZrO2, Zirconium Dioxide. https:// wWw. azom.com/properties. aspx?ArticleID=133. Version: 21.07.2021

[105] Aryan, A. ; Gulllet, B. ; Routoure, J. M. ; Fur, C. ; Langlois, P. ; MÉchin, L.: Measurement of thermal conductance of La0.7Sr0.3MnO3 thin films deposited on $\mathrm{SrTiO} 3$ and $\mathrm{MgO}$ substrates. In: Applied Surface Science 
326 (2015), 204-210. http://dx.doi.org/10.1016/j.apsusc.2014.11. 119. - DOI 10.1016/j.apsusc.2014.11.119. - ISSN 0169-4332

[106] Lazić, Ivan ; Bosch, Eric G. T. ; LazAR, Sorin: Phase contrast STEM for thin samples: Integrated differential phase contrast. In: Ultramicroscopy 160 (2016), 265-280. http://dx.doi.org/10.1016/j.ultramic. 2015.10.011. - DOI 10.1016/j.ultramic.2015.10.011. - ISSN 1879-2723

[107] Chmaissem, O. ; Dabrowski, B. ; Kolesnik, S. ; Mais, J. ; Brown, D. E. ; Kruk, R. ; Prior, P. ; Pyles, B. ; Jorgensen, J. D.: Relationship between structural parameters and the Néel temperature in $\mathrm{Sr} 1-\mathrm{xCaxMnO} 3(0<\sim \mathrm{x}<\sim 1)$ and $\mathrm{Sr} 1-\mathrm{yBayMnO} 3(\mathrm{y}<\sim 0.2)$. In: Physical review. B, Condensed matter 64 (2001), Nr. 13, S. 134412. http://dx.doi. org/10.1103/PhysRevB.64.134412. - DOI 10.1103/PhysRevB.64.134412. - ISSN 0163-1829

[108] Norby, P. ; Andersen, I.G.Krogh ; Andersen, E.Krogh ; Andersen, N. H.: The crystal structure of lanthanum manganate(iii), LaMnO3, at room temperature and at $1273 \mathrm{~K}$ under N2. In: Journal of Solid State Chemistry 119 (1995), Nr. 1, 191-196. http://dx.doi.org/10.1016/0022-4596(95) 80028-N. - DOI 10.1016/0022-4596(95)80028-N. - ISSN 0022-4596

[109] Cohn, J. L. ; Neumeier, J. J. ; PopoviCiu, C. P. ; MCClellan, K. J. ; LeVentourI, Th.: Local lattice distortions and thermal transport in perovskite manganites. In: Physical review. B, Condensed matter 56 (1997), Nr. 14, S. R8495-R8498. http://dx.doi .org/10.1103/PhysRevB.56.R8495. DOI 10.1103/PhysRevB.56.R8495. - ISSN 0163-1829

[110] Callaway, Joseph: Model for Lattice Thermal Conductivity at Low Temperatures. In: Physical Review 113 (1959), Nr. 4, S. 1046-1051. http://dx. doi.org/10.1103/PhysRev.113.1046. - DOI 10.1103/PhysRev.113.1046

[111] Asen-Palmer, M. ; Bartkowski, K. ; Gmelin, E. ; Cardona, M. ; Zhernov, A. P. ; Inyushkin, A. V. ; Taldenkov, A. ; Ozhogin, V. I. ; ITOH, K. M. ; Haller, E. E.: Thermal conductivity of germanium crystals with different isotopic compositions. In: Physical review. B, Conden- 
sed matter 56 (1997), Nr. 15, S. 9431-9447. http://dx.doi.org/10.1103/ PhysRevB.56.9431. - DOI 10.1103/PhysRevB.56.9431. - ISSN 0163-1829

[112] Zou, Jie ; Balandin, Alexander: Phonon heat conduction in a semiconductor nanowire. In: Journal of Applied Physics 89 (2001), Nr. 5, 29322938. http://dx.doi.org/10.1063/1.1345515. - DOI 10.1063/1.1345515. - ISSN 0021-8979

[113] Klemens, P. G.: The Scattering of Low-Frequency Lattice Waves by Static Imperfections. In: Proceedings of the Physical Society. Section A 68 (1955), Nr. 12, 1113-1128. http://dx.doi.org/10.1088/0370-1298/68/12/303. - DOI 10.1088/0370-1298/68/12/303. - ISSN 0370-1298

[114] Shayduk, Roman ; Navirian, Hengameh ; Leitenberger, Wolfram ; Goldshteyn, Jevgenij ; Vrejoiu, Ionela ; Weinelt, Martin ; GaAl, Peter ; Herzog, Marc ; Schmising, Clemens von K. ; BArgheer, Matias: Nanoscale heat transport studied by high-resolution time-resolved x-ray diffraction. In: New Journal of Physics 13 (2011), Nr. 9, 093032. http: //dx.doi.org/10.1088/1367-2630/13/9/093032. - DOI 10.1088/13672630/13/9/093032. - ISSN 1367-2630

[115] KIM, Jihoon ; PARK, Daeil ; NAGAno, Hosei ; TACHIKAWA, Sumitaka: Thermophysical Properties of Metal-Insulation Transition Materials as a Functional Thermal Control Device for Microsatellite. 47th International Conference on Environmental Systems, 2017 https://ttu-ir.tdl.org/handle/2346/ 73117

[116] Li, Junxue ; Simensen, Haakon T. ; Reitz, Derek ; Sun, Qiyang ; YuAn, Wei ; Li, Chen ; Tserkovnyak, Yaroslav ; BrataAs, Arne ; Shi, Jing: Observation of Magnon Polarons in a Uniaxial Antiferromagnetic Insulator. In: Physical Review Letters 125 (2020), Nr. 21, S. 217201. http: //dx.doi.org/10.1103/PhysRevLett.125.217201. - DOI 10.1103/PhysRevLett.125.217201. - ISSN 0031-9007

[117] Neighbours, J. R. ; Moss, R. W.: Ultrasonic attenuation near the critical point of $\operatorname{MnF}(2)$. (1968). https://calhoun.nps . edu/handle/10945/47512 
[118] Shapira, Y. ; Foner, S.: Magnetic Phase Diagram of Mn F2 from Ultrasonic and Differential Magnetization Measurements. In: Physical Review B 1 (1970), Nr. 7, S. 3083-3096. http://dx.doi.org/10.1103/PhysRevB.1. 3083. - DOI 10.1103/PhysRevB.1.3083

[119] Sheard, F. W.: Spin-Phonon Interactions. Version: 1976. http://dx. doi.org/10.1007/978-1-4613-4271-7\{_\}39. In: ChAllis, L. J. (Hrsg.) ; Rampton, V. W. (Hrsg.) ; Wyatt, A. F. G. (Hrsg.): Phonon Scattering in Solids. Boston, MA : Springer, 1976. - DOI 10.1007/978-1-4613-4271-7_39. - ISBN 978-1-4613-4271-7, S. 154-162

[120] Walton, D. ; Mook, H. A. ; Nicklow, R. M.: Neutron Inelastic Scattering by Coupled Defect-Phonon Modes in KCl-CN. In: Physical Review Letters 33 (1974), Nr. 7, S. 412-414. http://dx.doi.org/10.1103/PhysRevLett. 33.412. - DOI 10.1103/PhysRevLett.33.412. - ISSN 0031-9007

[121] Rezende, Sergio M. ; Azevedo, Antonio ; Rodríguez-Suárez, Roberto L.: Introduction to antiferromagnetic magnons. In: Journal of Applied Physics 126 (2019), Nr. 15, 151101. http://dx.doi.org/10.1063/1. 5109132. - DOI 10.1063/1.5109132. - ISSN 0021-8979

[122] Vansteenkiste, Arne ; Leliaert, Jonathan ; Dvornik, Mykola ; HelSen, Mathias ; Garcia-Sanchez, Felipe ; van Waeyenberge, Bartel: The design and verification of MuMax3. In: AIP Advances 4 (2014), Nr. 10, 107133. http://dx.doi.org/10.1063/1.4899186. - DOI $10.1063 / 1.4899186$

[123] Swartz, E. T. ; Pohl, R. O.: Thermal resistance at interfaces. In: Applied Physics Letters 51 (1987), Nr. 26, S. 2200-2202. http://dx.doi.org/10. 1063/1.98939. - DOI 10.1063/1.98939. - ISSN 0003-6951

[124] Stoner ; MARIS: Kapitza conductance and heat flow between solids at temperatures from 50 to $300 \mathrm{~K}$. In: Physical review. B, Condensed matter 48 (1993), Nr. 22, S. 16373-16387. http://dx.doi.org/10.1103/PhysRevB. 48.16373. - DOI 10.1103/PhysRevB.48.16373. - ISSN 0163-1829 
[125] Costescu, Ruxandra M. ; Wall, Marcel A. ; Cahill, David G.: Thermal conductance of epitaxial interfaces. In: Physical review. B, Condensed matter 67 (2003), Nr. 5, S. 054302. http://dx.doi.org/10.1103/PhysRevB. 67. 054302. - DOI 10.1103/PhysRevB.67.054302. - ISSN 0163-1829

[126] Gundrum, Bryan C. ; Cahill, David G. ; Averback, Robert S.: Thermal conductance of metal-metal interfaces. In: Physical Review B 72 (2005), Nr. 24, S. 245426. http://dx.doi.org/10.1103/PhysRevB.72.245426. - DOI 10.1103/PhysRevB.72.245426

[127] Young ; MARIS: Lattice-dynamical calculation of the Kapitza resistance between fcc lattices. In: Physical review. B, Condensed matter 40 (1989), Nr. 6, S. 3685-3693. http://dx.doi.org/10.1103/PhysRevB.40.3685. DOI 10.1103/PhysRevB.40.3685. - ISSN 0163-1829

[128] Swartz, E. T. ; Pohl, R. O.: Thermal boundary resistance. In: Rev. Mod. Phys. 61 (1989), Nr. 3, 605-668. http://dx.doi.org/10.1103/ RevModPhys.61.605. - DOI 10.1103/RevModPhys.61.605

[129] Togo, Atsushi ; TANAKA, Isao: First principles phonon calculations in materials science. In: Scripta Materialia 108 (2015), 15. http://dx.doi.org/10.1016/j.scriptamat.2015.07.021. - DOI 10.1016/j.scriptamat.2015.07.021. - ISSN 1359-6462

[130] Aytan, E. ; Debnath, B. ; Kargar, F. ; Barlas, Y. ; Lacerda, M. M. ; Li, J. X. ; Lake, R. K. ; Shi, J. ; Balandin, A. A.: Spin-phonon coupling in antiferromagnetic nickel oxide. In: Applied Physics Letters 111 (2017), Nr. 25, 252402. http://dx.doi.org/10.1063/1.5009598. - DOI 10.1063/1.5009598. - ISSN 0003-6951

[131] Sebastian, Thomas ; Schultheiss, Katrin ; Obry, Björn ; Hillebrands, Burkard ; Schultheiss, Helmut: Micro-focused Brillouin light scattering: imaging spin waves at the nanoscale. In: Frontiers in Physics 3 (2015). http: //dx.doi.org/10.3389/fphy.2015.00035. - DOI 10.3389/fphy.2015.00035

[132] Cahill, D. G. ; Pohl, R. O.: Lattice Vibrations and Heat Transport in Crystals and Glasses. In: Annual Review of Physical Chemistry 39 (1988), 
Nr. 1, S. 93-121. http://dx.doi.org/10.1146/annurev.pc.39.100188. 000521. - DOI 10.1146/annurev.pc.39.100188.000521. - ISSN 0066-426X

[133] Cahill ; Watson ; Pohl: Lower limit to the thermal conductivity of disordered crystals. In: Physical review. B, Condensed matter 46 (1992), Nr. 10, S. 6131-6140. http://dx.doi.org/10.1103/PhysRevB.46.6131. - DOI 10.1103/PhysRevB.46.6131. - ISSN 0163-1829

[134] Mizuno, Hideyuki ; Mossa, Stefano ; Barrat, Jean-Louis: Beating the amorphous limit in thermal conductivity by superlattices design. In: Scientific Reports 5 (2015), Nr. 1, 14116. http://dx.doi.org/10.1038/ srep14116. - DOI 10.1038/srep14116. - ISSN 2045-2322

[135] CAPs, R. ; Fricke, J.: Aerogels for Thermal Insulation. Version: 2004. http://dx.doi.org/10.1007/978-0-387-88953-5\{_\}46. In: AEGERTER, Michel A. (Hrsg.) ; Mennig, Martin (Hrsg.): Sol-Gel Technologies for Glass Producers and Users. Boston, MA and s.l. : Springer US, 2004. - DOI 10.1007/978-0-387-88953-5_46. - ISBN 978-0-387-88953-5, S. 349-353

[136] Meyer, Dennis ; Metternich, Daniel ; Henning, Pia ; Bange, Jan P. ; Gruhl, Robert ; Bruchmann-Bamberg, Vitaly ; Moshnyaga, Vasily ; UlRICHS, Henning: Coherent phonon transport and minimum of thermal conductivity in $\mathrm{LaMnO}_{3} / \mathrm{SrMnO}_{3}$ superlattices. https://arxiv.org/pdf/ 2108.05860

[137] Binder, Harry H.: Lexikon der chemischen Elemente: Das Periodensystem in Fakten, Zahlen und Daten; mit vielen tabellarischen Zusammenstellungen. Stuttgart and Leipzig : Hirzel, 1999. - ISBN 3777607363

[138] Ghivelder, L. ; Abrego Castillo, I. ; Gusmão, M. A. ; Alonso, J. A. ; Cohen, L. F.: Specific heat and magnetic order in LaMnO3. In: Physical review. B, Condensed matter 60 (1999), Nr. 17, S. 12184-12190. http://dx.doi.org/10.1103/PhysRevB.60.12184. - DOI 10.1103/PhysRevB.60.12184. - ISSN 0163-1829

[139] Choithrani, Renu ; Gaur, N. K. ; Singh, R. K.: Specific heat and transport properties of La1-xGdxMnO3 at. In: Solid State Communications 147 
(2008), Nr. 3-4, 103-106. http://dx.doi.org/10.1016/j.ssc.2008.05. 005. - DOI 10.1016/j.ssc.2008.05.005. - ISSN 0038-1098

[140] Jang, Yun H. ; Gervais, François ; LansaC, Yves: A-site ordering in colossal magnetoresistance manganite $\mathrm{La}(1-\mathrm{x}) \mathrm{Sr}(\mathrm{x}) \mathrm{MnO}$ ? Molecular dynamics simulations and quantum mechanics calculations. In: The Journal of Chemical Physics 131 (2009), Nr. 9, 094503. http://dx.doi.org/10.1063/1. 3190533. - DOI 10.1063/1.3190533. - ISSN 0021-9606

[141] Rini, E. G. ; RaO, Mala N. ; Chaplot, S. L. ; Gaur, N. K. ; Singh, R. K.: Phonon dynamics of lanthanum manganite LaMnO3 using an interatomic shell model potential. In: Physical Review B 75 (2007), Nr. 21, S. 214301. http://dx.doi.org/10.1103/PhysRevB.75.214301. - DOI 10.1103/PhysRevB.75.214301

[142] Reichardt, W. ; Braden, M.: Anomalous features in the bond stretching vibrations of La1-xSrxMnO3. In: Physica B Condensed Matter 263-264 (1999), Nr. 1-4, S. 416-420. http://dx.doi.org/10.1016/S0921-4526(98) 01400-8. - DOI 10.1016/S0921-4526(98)01400-8. - ISSN 0921-4526

[143] Zinenko, V. I. ; PAVlovskil̆, M. S.: Lattice dynamics and the phase transition from the cubic phase to the tetragonal phase in the LaMnO3 crystal within the polarizable-ion model. In: Physics of the Solid State 49 (2007), Nr. 9, 1749-1758. http://dx.doi.org/10.1134/S1063783407090235. - DOI 10.1134/S1063783407090235. - ISSN 1090-6460

[144] Sakai, H. ; Fujioka, J. ; Fukuda, T. ; Bahramy, M. S. ; OKuyama, D. ; Arita, R. ; Arima, T. ; Baron, A. Q. R. ; Taguchi, Y. ; Tokura, Y.: Soft phonon mode coupled with antiferromagnetic order in incipient-ferroelectric Mott insulators Sr 1-x Ba x MnO 3. In: Physical Review B 86 (2012), Nr. 10, S. 104407. http://dx.doi.org/10.1103/PhysRevB.86.104407. - DOI 10.1103/PhysRevB.86.104407

[145] Ren, Yunpeng ; Chen, J. K. ; Zhang, Yuwen: Optical properties and thermal response of copper films induced by ultrashort-pulsed lasers. In: Journal of Applied Physics 110 (2011), Nr. 11, 113102. http://dx.doi. org/10.1063/1.3662897. - DOI 10.1063/1.3662897. - ISSN 0021-8979 
[146] Ashkenasi, D. ; Rosenfeld, A. ; Varel, H. ; Wähmer, M. ; Campbell, E.E.B: Laser processing of sapphire with picosecond and sub-picosecond pulses. In: Applied Surface Science 120 (1997), Nr. 1-2, 65-80. http: //dx.doi.org/10.1016/S0169-4332(97)00218-3. - DOI 10.1016/S01694332(97)00218-3. - ISSN 0169-4332

[147] Lenzner, M. ; Krausz, F. ; Krüger, J. ; Kautek, W.: Photoablation with sub-10 fs laser pulses. In: Applied Surface Science 154-155 (2000), 11-16. http://dx.doi.org/10.1016/S0169-4332(99)00432-8. - DOI 10.1016/S0169-4332(99)00432-8. - ISSN 0169-4332

[148] Zemaitis, Andrius ; Gecys, Paulius ; Barkauskas, Martynas ; RaCiuKAItis, Gediminas ; Gedvilas, Mindaugas: Highly-efficient laser ablation of copper by bursts of ultrashort tuneable (fs-ps) pulses. In: Scientific Reports 9 (2019), Nr. 1, 12280. http://dx.doi.org/10.1038/s41598-019-48779-w. - DOI 10.1038/s41598-019-48779-w. - ISSN 2045-2322

[149] LU, Chih-Shun ; LEWIS, Owen: Investigation of film-thickness determination by oscillating quartz resonators with large mass load. In: Journal of Applied Physics 43 (1972), Nr. 11, 4385-4390. http://dx.doi.org/10.1063/ 1.1660931. - DOI 10.1063/1.1660931. - ISSN 0021-8979 



\section{Anhang $A$}

\section{Anhang}

\section{A.1 Materialparameter}

Tabelle A.1: Für Simulation und Berechnungen verwendete Materialparameter.

Der Wert für $\kappa_{\mathrm{LMO}}$ und $\kappa_{\mathrm{LSMO}}$ wurde experimentell bestimmt.

\begin{tabular}{|c|c|c|c|c|}
\hline & $\mathrm{C}_{\mathrm{p}}\left[\mathrm{J} \mathrm{kg}^{-1} \mathrm{~K}^{-1}\right]$ & $\rho\left[\mathrm{kg} \mathrm{m}^{-3}\right]$ & $v_{\mathrm{L}}\left[\mathrm{m} \mathrm{s}^{-1}\right]$ & $\kappa\left[\mathrm{W} \mathrm{m}^{-1} \mathrm{~K}^{-1}\right]$ \\
\hline \hline $\mathrm{W}[26]$ & 126 & 19300 & 5220 & 130 \\
\hline $\mathrm{MgO}[26]$ & 520 & 3580 & 8700 & 12 \\
\hline $\mathrm{ZrO}_{2}[26]$ & 540 & 6120 & 6300 & 2 \\
\hline Si-Substrat[73, 137] & 703 & 2392 & 8433 & 149 \\
\hline SiOx-Substrat[73] & 700 & 2200 & - & 1.4 \\
\hline $\mathrm{Cu}[73,137]$ & 385 & 8960 & - & 400 \\
\hline $\mathrm{LaMnO}_{3}$ & $487[138,139]$ & $6520[82]$ & - & $1.3(\exp )$. \\
\hline $\mathrm{SrMnO}_{3}$ & $540[81]$ & $5460[82]$ & - & $4.5[101]$ \\
\hline $\mathrm{Sr}_{0.5} \mathrm{La}_{0.5} \mathrm{MnO}_{3}[109,114,115]$ & 532 & 6500 & - & $2-2.6(\exp )$. \\
\hline $\mathrm{STO}_{2} \mathrm{Substrat}_{1114}$ & 544 & 5110 & - & 12 \\
\hline
\end{tabular}




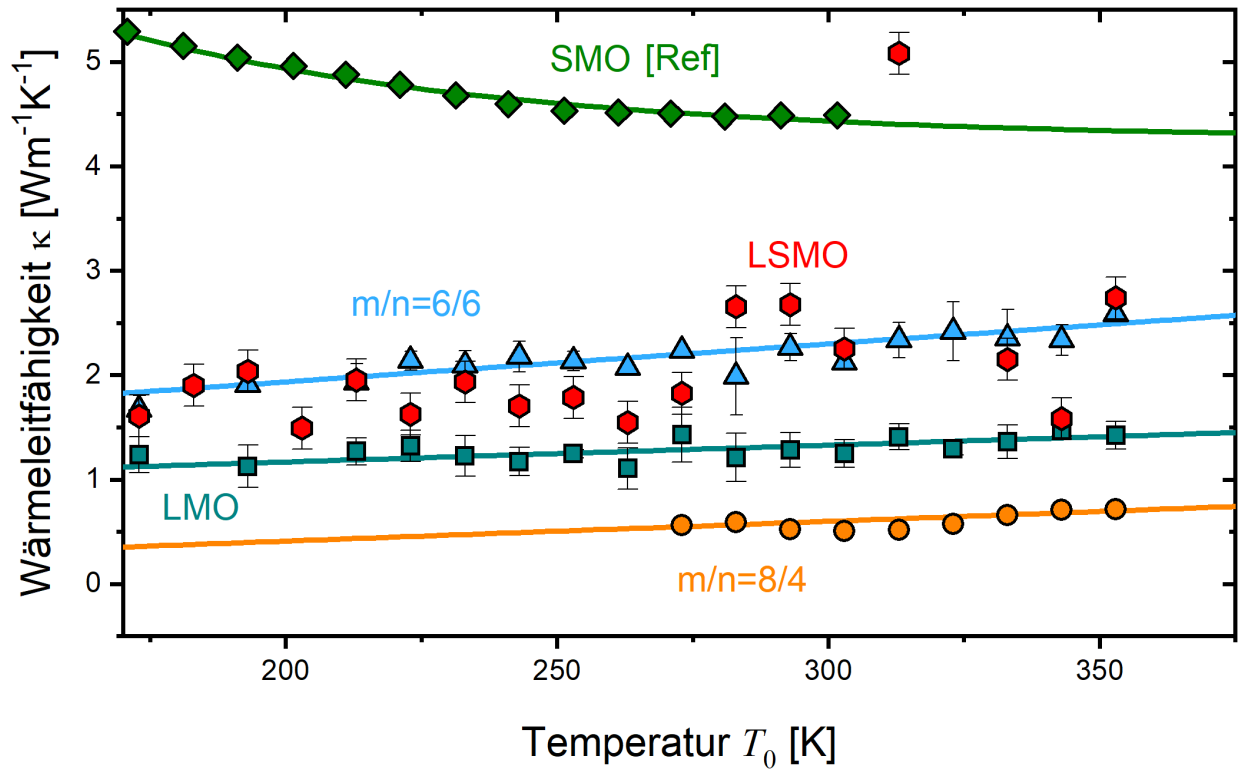

Abbildung A.1: TTTR Referenzmessungen an $\mathrm{LMO}_{6} / \mathrm{SMO}_{6}$ (blau), $\mathrm{LMO}_{8} / \mathrm{SMO}_{4}$ (orange), LMO (grün) und $\mathrm{La}_{0.5} \mathrm{Sr}_{0.5} \mathrm{MnO}_{3}$ (rot). Die SMO Werte stammen aus [101].

Die Grenzflächenrauhigkeiten für $\mathrm{LMO}_{10} / \mathrm{SMO}_{5}$ und $\mathrm{LMO}_{5} / \mathrm{SMO}_{5}$ sind in Tabelle A.2 aufgeführt. 
Tabelle A.2: Grenzflächenrauhigkeiten (RMS) für $\mathrm{LMO}_{10} / \mathrm{SMO}_{5}$ und $\mathrm{LMO}_{5} / \mathrm{SMO}_{5}$. Die Werte wurden mit Hilfe von Röntgenreflektometrie (XRR) und Röntgendiffraktometrie (XRD) bestimmt. Details wie die Rauhigkeit aus den Röntgendaten bestimmt werden kann sind in [80] zu finden.

\begin{tabular}{|l|c|c|}
\hline & $\mathrm{LMO}_{10} / \mathrm{SMO}_{5}$ & $\mathrm{LMO}_{5} / \mathrm{SMO}_{5}$ \\
\hline XRR STO/ÜG & $1.1(1) \mathrm{nm}$ & $1.0(3) \mathrm{nm}$ \\
\hline XRR LMO/SMO & $0.3(2) \mathrm{nm}$ & $0.3(1) \mathrm{nm}$ \\
\hline XRR SMO/LMO & $0.5(2) \mathrm{nm}$ & $0.3(1) \mathrm{nm}$ \\
\hline XRR ÜG Oberfläche & $0.4(1) \mathrm{nm}$ & $0.3(1) \mathrm{nm}$ \\
\hline XRD STO/ÜG & $1.0(5) \mathrm{nm}$ & $1.0(5) \mathrm{nm}$ \\
\hline XRD LMO/SMO & $0.3(2) \mathrm{nm}$ & $0.2(1) \mathrm{nm}$ \\
\hline XRD SMO/LMO & $0.6(3) \mathrm{nm}$ & $0.4(3) \mathrm{nm}$ \\
\hline XRD ÜG Oberfläche & $0.6(4) \mathrm{nm}$ & $0.5(3) \mathrm{nm}$ \\
\hline
\end{tabular}

Tabelle A.3: Kraftkonstanten und Schallgeschwindigkeiten im BK-Modell.

\begin{tabular}{|c|c|c|c|c|c|}
\hline & $\mathrm{D}_{1}\left[\mathrm{~N} \mathrm{~m}^{-1}\right]$ & $\mathrm{D}_{2}\left[\mathrm{~N} \mathrm{~m}^{-1}\right]$ & $\mathrm{D}_{3}\left[\mathrm{~N} \mathrm{~m}^{-1}\right]$ & $v_{\mathrm{LA}}\left[\mathrm{m} \mathrm{s}^{-1}\right]$ & $v_{\mathrm{TA}}\left[\mathrm{m} \mathrm{s}^{-1}\right]$ \\
\hline SMO $(\mathrm{Pm} \overline{3} \mathrm{~m})$ & 13.13 & 18.94 & 17.51 & 5723 & 4432 \\
\hline LMO $(\mathrm{Pm} \overline{3} \mathrm{~m})$ & 60.08 & 50.24 & 8.16 & 7754 & 4737 \\
\hline LMO $(\mathrm{R} \overline{3} \mathrm{c})$ & 36.31 & 28.13 & 0 & 5698 & 3217 \\
\hline
\end{tabular}

Die Kraftkonstanten für kubisches SMO (Pm̄̄m) stammen aus DFT-Rechnungen[81]. Anhand von MD-Potentialen[140] wurde das Phononenspektrum in der harmonischen Näherung für kubisches LMO (Pm̄̄m) berechnet. Die Kraftkonstanten wurden dann durch Anpassen des BKM-Spektrum an dieses Spektrum bestimmt. Die Kraftkonstanten für rhomboedrisches LMO wurden aus elastischen Konstanten[141] berechnet. Mit den Kraftkonstanten in Tabelle A.3 und unserem BK-Modell berechnete Phononenspektren reproduzieren experimentell bestimmte longitudinale und transversale Schallgeschwindigkeiten [142-144]. 


\section{A.2 Determinaten zur Herleitung der Rytov-Gleichung}

Im Folgenden beschreibe ich das Lösen der Determinaten $\operatorname{det} M$ zur Herleitung der Rytov-Formel in der Form wie in Gleichung 2.10.

$$
\operatorname{det} M=a_{1,1} \operatorname{det} M_{1}-a_{1,2} \operatorname{det} M_{2}+a_{1,3} \operatorname{det} M_{3}-a_{1,4} \operatorname{det} M_{4}
$$

mit den Summanden

$$
\begin{aligned}
& a_{1,1} \operatorname{det} M_{1}=1 \cdot\left|\begin{array}{ccc}
e^{-\beta_{1}} & -1 & -1 \\
-\gamma_{1} & -\gamma_{2} e^{\beta_{2}} e^{-i Q d} & \gamma_{2} e^{-\beta_{2}} e^{-i Q d} \\
-\gamma_{1} e^{-\beta_{1}} & -\gamma_{2} & \gamma_{2}
\end{array}\right| \\
& =e^{-\beta_{1}} \cdot\left(-\gamma_{2} e^{\beta_{2}} e^{-i Q d}\right) \cdot \gamma_{2} \\
& +(-1) \cdot \gamma_{2} e^{-\beta_{2}} e^{-i Q d} \cdot\left(-\gamma_{1} e^{-\beta_{1}}\right) \\
& +(-1) \cdot\left(-\gamma_{1}\right) \cdot\left(-\gamma_{2}\right) \\
& -\left(-\gamma_{1} e^{-\beta_{1}}\right)\left(-\gamma_{2} e^{\beta_{2}} e^{-i Q d}\right)(-1) \\
& -\left(-\gamma_{2}\right)\left(\gamma_{2} e^{-\beta_{2}} e^{-i Q d}\right)\left(e^{-\beta_{1}}\right) \\
& -\left(\gamma_{2}\right)\left(-\gamma_{1}\right)(-1) \text {, } \\
& a_{1,2} \operatorname{det} M_{2}=1 \cdot\left|\begin{array}{ccc}
e^{\beta_{1}} & -1 & -1 \\
\gamma_{1} & -\gamma_{2} e^{\beta_{2}} e^{-i Q d} & \gamma_{2} e^{-\beta_{2}} e^{-i Q d} \\
\gamma_{1} e^{\beta_{1}} & -\gamma_{2} & \gamma_{2}
\end{array}\right| \\
& =e^{\beta_{1}}\left(-\gamma_{2} e^{\beta_{2}} e^{-i Q d}\right) \gamma_{2} \\
& +(-1)\left(\gamma_{2} e^{-\beta_{2}} e^{-i Q d}\right)\left(\gamma_{1} e^{\beta_{1}}\right) \\
& +(-1)\left(\gamma_{1}\right)\left(-\gamma_{2}\right) \\
& -\left(\gamma_{1} e^{\beta_{1}}\right)\left(-\gamma_{2} e^{\beta_{2}} e^{-i Q d}\right)(-1) \\
& -\left(-\gamma_{2}\right)\left(\gamma_{2} e^{-\beta_{2}} e^{-i Q d}\right)\left(e^{\beta_{1}}\right) \\
& -\left(\gamma_{2}\right)\left(\gamma_{1}\right)(-1) \text {, }
\end{aligned}
$$




$$
\begin{aligned}
& a_{1,3} \operatorname{det} M_{3}=-e^{\beta_{2}} e^{-i Q d} \cdot\left|\begin{array}{ccc}
e^{\beta_{1}} & e^{-\beta_{1}} & -1 \\
\gamma_{1} & -\gamma_{1} & \gamma_{2} e^{-\beta_{2}} e^{-i Q d} \\
\gamma_{1} e^{\beta_{1}} & -\gamma_{1} e^{-\beta_{1}} & \gamma_{2}
\end{array}\right| \\
& =\left(e^{\beta_{1}}\right)\left(-\gamma_{1}\right)\left(\gamma_{2}\right)\left(-e^{\beta_{2}} e^{-i Q d}\right) \\
& +\left(e^{-\beta_{1}}\right)\left(\gamma_{2} e^{-\beta_{2}} e^{-i Q d}\right)\left(\gamma_{1} e^{\beta_{1}}\right)\left(-e^{\beta_{2}} e^{-i Q d}\right) \\
& +(-1)\left(\gamma_{1}\right)\left(-\gamma_{1} e^{-\beta_{1}}\right)\left(-e^{\beta_{2}} e^{-i Q d}\right) \\
& -\left(\gamma_{1} e^{\beta_{1}}\right)\left(-\gamma_{1}\right)(-1)\left(-e^{\beta_{2}} e^{-i Q d}\right) \\
& -\left(-\gamma_{1} e^{-\beta_{1}}\right)\left(\gamma_{2} e^{-\beta_{2}} e^{-i Q d}\right)\left(e^{\beta_{1}}\right)\left(-e^{\beta_{2}} e^{-i Q d}\right) \\
& -\left(\gamma_{2}\right)\left(\gamma_{1}\right)\left(e^{-\beta_{1}}\right)\left(-e^{\beta_{2}} e^{-i Q d}\right) \text {, } \\
& a_{1,4} \operatorname{det} M_{4}=-e^{-\beta_{2}} e^{-i Q d} \cdot\left|\begin{array}{ccc}
e^{\beta_{1}} & e^{-\beta_{1}} & -1 \\
\gamma_{1} & -\gamma_{1} & -\gamma_{2} e^{\beta_{2}} e^{-i Q d} \\
\gamma_{1} e^{\beta_{1}} & -\gamma_{1} e^{-\beta_{1}} & -\gamma_{2}
\end{array}\right| \\
& =\left(e^{\beta_{1}}\right)\left(-\gamma_{1}\right)\left(-\gamma_{2}\right)\left(-e^{-\beta_{2}} e^{-i Q d}\right) \\
& +\left(e^{-\beta_{1}}\right)\left(-\gamma_{2} e^{\beta_{2}} e^{-i Q d}\right)\left(\gamma_{1} e^{\beta_{1}}\right)\left(-e^{-\beta_{2}} e^{-i Q d}\right) \\
& +(-1)\left(\gamma_{1}\right)\left(-\gamma_{1} e^{-\beta_{1}}\right)\left(-e^{-\beta_{2}} e^{-i Q d}\right) \\
& -\left(\gamma_{1} e^{\beta_{1}}\right)\left(-\gamma_{1}\right)(-1)\left(-e^{-\beta_{2}} e^{-i Q d}\right) \\
& -\left(-\gamma_{1} e^{-\beta_{1}}\right)\left(-\gamma_{2} e^{\beta_{2}} e^{-i Q d}\right)\left(e^{\beta_{1}}\right)\left(-e^{-\beta_{2}} e^{-i Q d}\right) \\
& -\left(-\gamma_{2}\right)\left(\gamma_{1}\right)\left(e^{-\beta_{1}}\right)\left(-e^{-\beta_{2}} e^{-i Q d}\right)
\end{aligned}
$$

Nach Ausmultiplizieren erhalten wir:

$$
\begin{aligned}
\operatorname{det} M & =2 \gamma_{1} \gamma_{2}\left(e^{\beta_{1}} e^{\beta_{2}}+e^{-\beta_{1}} e^{-\beta_{2}}+e^{-\beta_{1}} e^{\beta_{2}}+e^{\beta_{1}} e^{-\beta_{2}}\right) e^{-i Q d} \\
& -\gamma_{1}^{2}\left(e^{\beta_{1}} e^{\beta_{2}}+e^{-\beta_{1}} e^{-\beta_{2}}-e^{-\beta_{1}} e^{\beta_{2}}-e^{\beta_{1}} e^{-\beta_{2}}\right) e^{-i Q d} \\
& -\gamma_{2}^{2}\left(e^{\beta_{1}} e^{\beta_{2}}+e^{-\beta_{1}} e^{-\beta_{2}}-e^{-\beta_{1}} e^{\beta_{2}}-e^{\beta_{1}} e^{-\beta_{2}}\right) e^{-i Q d} \\
& -4 \gamma_{1} \gamma_{2} e^{-2 i Q d} \\
& -4 \gamma_{1} \gamma_{2} \\
& =0
\end{aligned}
$$


Mit den Identitäten $e^{a} e^{b}+e^{-a} e^{-b}+e^{a} e^{-b}+e^{-a} e^{b}=4 \cosh (a) \cosh (b)$ und $e^{a} e^{b}+$ $e^{-a} e^{-b}-e^{a} e^{-b}-e^{-a} e^{b}=4 \sinh (a) \sinh (b)$ und Addition von $4 \gamma_{1} \gamma_{2}+4 \gamma_{1} \gamma_{2} e^{-2 i Q d}$ :

$$
\begin{aligned}
\Leftrightarrow \quad & 8 \gamma_{1} \gamma_{2} \cosh \left(\beta_{1}\right) \cosh \left(\beta_{2}\right) e^{-i Q d} \\
& -4 \gamma_{1}^{2} \sinh \left(\beta_{1}\right) \sinh \left(\beta_{2}\right) e^{-i Q d} \\
& -4 \gamma_{2}^{2} \sinh \left(\beta_{1}\right) \sinh \left(\beta_{2}\right) e^{-i Q d} \\
& =4 \gamma_{1} \gamma_{2}+4 \gamma_{1} \gamma_{2} e^{-2 i Q d}
\end{aligned}
$$

Geteilt durch $8 \gamma_{1} \gamma_{2} e^{-i Q d}$ und zusammenfassen:

$$
\begin{gathered}
\Leftrightarrow \quad \cosh \left(\beta_{1}\right) \cosh \left(\beta_{2}\right)-\frac{1}{2}\left(\frac{\gamma_{1}^{2}}{\gamma_{1} \gamma_{2}}+\frac{\gamma_{2}^{2}}{\gamma_{1} \gamma_{2}}\right) \sinh \left(\beta_{1}\right) \sinh \left(\beta_{2}\right) \\
=\frac{1}{2}\left(e^{i Q d}+e^{-i Q d}\right)=\cosh (i Q d)
\end{gathered}
$$


Einsetzen von $\alpha_{i}=\frac{\omega}{v_{i}}, \beta_{i}=i \alpha_{i} d_{i}$ und $\gamma_{i}=\alpha_{i} \rho_{i} v_{i}^{2}$ und Ausnutzen der Beziehung $\cosh (i a)=\cos (a)$ und $\sinh (i a)=i \cdot \sin (a)$ :

$$
\cos \left(\frac{\omega d_{1}}{v_{1}}\right) \cos \left(\frac{\omega d_{2}}{v_{2}}\right)-\frac{1}{2}\left(\frac{\rho_{1} v_{1}}{\rho_{2} v_{2}}+\frac{\rho_{2} v_{2}}{\rho_{1} v_{1}}\right) \cdot \sin \left(\frac{\omega d_{1}}{v_{1}}\right) \cdot \sin \left(\frac{\omega d_{2}}{v_{2}}\right)=\cos (Q d)
$$

\section{A.3 TTR-Aufbau}

\section{A.3.1 Entwicklung des TTR-Aufbaus}

(a)

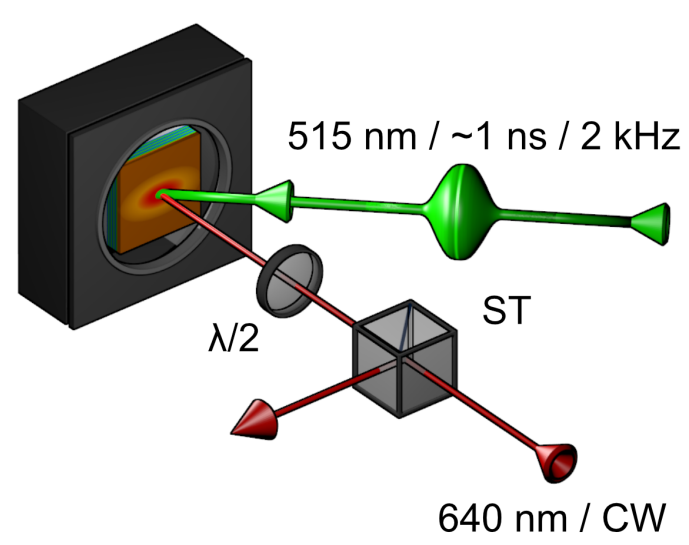

(b)

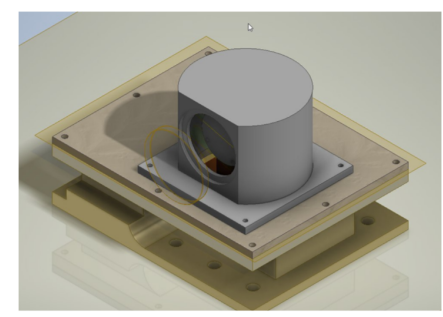

(c)

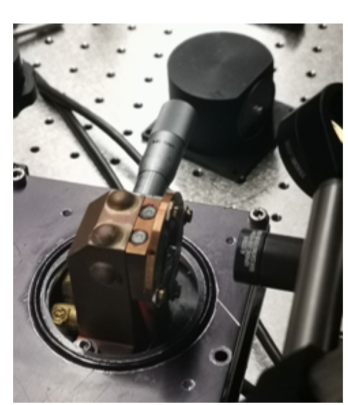

Abbildung A.2: (a) Vereinfachte Skizze des TTR mit den wichtigsten Daten der einzelnen Strahlen. ST = Strahlteiler. (b) CAD-Bild aus der Planungsphase des TTR-Kryo-Probenhalters. Zu sehen ist die Grundplatte mit der Aufsatzhaube und das Laserfenster. (c) Bild des Probenhalters ohne Haube (ist im Hintergrund zu sehen).

Diesen für die in dieser Arbeit gemessenen Wärmeleitfähigkeiten genutzten Aufbau habe ich zusammen mit meinen Masteranten Roland Potthast und Daniel Metternich aufgebaut. Bevor ich den finalen Aufbau darstelle, möchte ich kurz die wichtigsten Entwicklungsschritte des Aufbaus kurz skizzieren. 
Vom Aufbau der Gruppe von Prof. Dr. Krebs konnten wir den Diodenlaser, das Oszilloskop und ein in Visual Basic geschriebenes Aufnahmeprogramm für Kurven vom Oszilloskop übernehmen. Als Pumplaser haben wir zunächst einen $1030 \mathrm{~nm}$ Femtosekunden Laser im Labor der Gruppe von Prof. Dr. Mathias verwendet. In dieser Konfiguration konnte auf dem Oszilloskop keine Reflektivitätsänderung der Kupferfilme beobachtet werden. Die Referenzen [145-148] legen nahe, dass die ultrakurzen Pulse bei der nötigen Energie zum Heizen des Kupferfilms aufgrund der zeitlichen Kürze der Absorption zu einer lokalen Ablation des Kupfer anstatt zu einem gleichmäßigen Erwärmen führen. Die Intaktheit des Kupferfilms müssen wir jedoch für ein erfolgreiches TTR-Experiment voraussetzen.

In einem nächsten Schritt haben wir zur Anregung einen durchstimmbaren nsLaser (Opotek Opolette 355 II) verwendet. Der Laser wurde uns freundlicherweise von Prof. Dr. Jooss zur Verfügung gestellt. Mit diesem Laser konnten wir bei einer Pulslänge von $5 \mathrm{~ns}$, einer Wellenlänge von $450 \mathrm{~nm}$ und einer Pulsenergie laut Datenblatt von $5 \mathrm{~mJ}$ eine Änderung der Reflektivität beobachten. Auch wenn mit diesem Setup erste TTR-Kurven aufgenommen und ausgewertet werden konnten, war die niedrige Repititionsrate von $20 \mathrm{~Hz}$ des Pumplasers ein großes Problem für die Messung. Die Spannung des CW-Lasers auf der Messdiode wurde auf etwa 1.2 V bis $1.5 \mathrm{~V}$ eingestellt, da die Diode laut Datenblatt in diesem Bereich gerade noch eine lineare Abhängigkeit zwischen Laserfluenz und Photospannung zeigt. Die maximale Änderung der Reflektivität auf dem Kupferfilm liegt jedoch typischerweise nur bei einigen Millivolt. Erst durch das Trennen von Spannungshintergrund und Signaländerung über einen DC-Filter (Eingangsfilter am Oszilloskop) ist die Reflektivitätsänderung überhaupt sichtbar. Das niedrige Signal-zu-Rausch-Verhältnis hat eine hohe Kurvenmittelung am Oszilloskop nötig gemacht (65536 Mittelungen). Diese Anzahl an Messungen bei einer Repititionsrate von $20 \mathrm{~Hz}$ führt dazu, dass die Aufnahme einer einzelnen Messkurve fast eine Stunde dauert. Äußere Schwankungen wie Raumtemperatur, Stabilität der Laserquellen, usw. können über diese Zeit zu einem Driften des Messsignals führen, was die Qualität der Daten weiter vermindert. 


\section{A.3.2 TTR-Messanleitung}

An dieser Stelle möchte ich noch kurz den typischen Ablauf einer temperaturabhängigen TTR-Messung schildern und die wichtigsten Sicherheitsvorkehrungen ansprechen.

Aufgrund der Intensität der ns-Pulse $\left(>0.7 \mathrm{~J} \mathrm{~cm}^{-2}\right)$ und der hohen CW-Leistung $(\approx 50 \mathrm{~mW})$ muss zur Messung eine geeignete Laserschutzbrille getragen werden. Zur Justage kann der CW-Laser auf $<1 \mathrm{~mW}$ (Channel 2 aus) reduziert werden. Der Aufbau ist auf einer Lochrasterplatte aufgebaut und soweit möglich auf horizontaler Ebene von Laserschutzwänden umgeben.

Die Schritte einer erfolgreichen TTR-Messung sind im Folgenden aufgeführt:

1. Die (verkupferte) Probe wird auf dem Probenteller mit einem Bügel befestigt. Der Teller wird anschließend in den Kupferturm des Probenhalters geschraubt und das Pt1000 wird mit einer Schraube am Teller fixiert.

2. Die Haube wird über den Turm gesetzt und über den Pumpstand wird die Kammer auf etwa $5 \times 10^{-5}$ mbar- $1 \times 10^{-4}$ mbar abgepumpt. Dieser Schritt kann bei Messungen bei Raumtemperatur übersprungen werden.

3. Der CW-Laser wird auf niedriger Leistung (Kanal 1) angeschaltet und über den X-Y-Z Positionierer wird der Spot auf den Kupferfilm der Probe ausgerichtet. Der reflektierte Strahl sollte den linken Eingang der Photodiode treffen. Die optische Justage des TTR-Aufbaus hat sich als über lange Zeit stabil herausgestellt. Ein Nachjustieren der Strahlengänge ist selten nötig.

4. Der CW-Laser wird auf $>50 \mathrm{~mW}$ gestellt (Kanal 2). Bei niedrigeren Leistungen ist der Toptica Diodenlaser nicht leistungsstabil. An der Photodiode (mit verdecktem rechtem Detektor) sollten nun etwa $1.0 \mathrm{~V}$ bis $1.2 \mathrm{~V}$ abfallen. Dies kann am Oszilloskop abgelesen werden, wenn im Messprogramm der Button Adjustmode aktiviert wird ${ }^{1}$. Ist dies nicht der Fall, kann über eine $\lambda / 2$-Wellenplatte der Anteil des Lasers, der am zentralen Beamsplitter ausgeworfen wird verändert werden. Hinter der Objektivlinse sollten in etwa $5 \mathrm{~mW}$ ankommen. Mit einem Polarisator lässt sich die Leistung auf der

\footnotetext{
${ }^{1}$ Das Oszi schaltet dann in den DC gekoppelten Modus und die Mittelung wird deaktiviert.
} 
Photodiode noch genauer einstellen. 1.2 V haben sich als guter Kompromiss zwischen großer Signalamplitude und Linearität des Photodetektors herausgestellt. Soll die Messung als Differenzmessung erfolgen, muss an dieser Stelle der am Beamsplitter getrennte Strahl ebenfalls auf 1.2 V eingestellt und auf den rechten Port der Photodiode justiert werden. Dieser Schritt ist in der Regel nicht nötig, denn die AC-Filterung des Oszilloskops ist ausreichend, um den Reflextionshintergrund des Lasers vom Messsignal zu trennen.

5. Nun wird der ns-Pumplaser aktiviert. Auch hier ist in der Regel keine Justage des Strahlengangs nötig. Auf einem Monitor lässt sich das Bild einer OffAxis CCD-Kamera sehen, welche auf die Probe gerichtet ist und Pumpund Probestrahl abbilden kann. Auf diesem Bild kann der Überlapp der beiden Strahle eingestellt und der Fokus des Probestrahls justiert werden. Der Pumpstrahl wird relativ zum Probestrahl mit dem letzten Spiegel im Probestrahlengang bewegt. Schaltet man das Oszilloskop im Messprogramm auf Measuremode ${ }^{2}$, sollte auf dem Oszilloskop bei hinreichendem Überlapp eine TTR-Kurve zu sehen sein. Diese kann durch Verbessern des Überlapps noch maximiert werden.

6. Im Messprogramm kann auf Wunsch eine Temperaturliste angelegt oder aus einer Textdatei geladen werden. Mit Klick auf Start Measurement wird die Temperatursteuerung aktiviert und die erste Temperatur angefahren. Hat sich die Temperatur auf dem Zielwert eingeschwungen, läuft folgendes Messprotokoll ab:

a) Das Oszilloskop wird in den DC-Modus bei maximaler vertikaler Auflösung versetzt und es wird eine gemittelte Kurve aus 65536 einzelnen Messungen (eine pro Pump-Puls) aufgenommen. Der Durchschnittswert dieser Messungen wird als Referenzwert der Gesamtreflektivität gespeichert.

b) Das Oszilloskop wird zurück in den AC-Modus geschaltet und es werden $n$ Messkurven mit 65536 Samples aufgenommen. Im Messprogramm wird die letzte Kurve, sowie die Standardabweichung aus den vorheri-

${ }^{2} \mathrm{Im}$ Measuremode wird die Eingangkopplung des Oszilloskops in den AC-Modus und das Averaging auf 65536 Samples gestellt. Dazu wird die vertikale Auflösung auf $10 \mathrm{mV}$ reduziert. 
gen, bei der aktuellen Temperatur aufgenommenen Messungen angezeigt.

c) Alle Messkurven sowie die gemittelte Kurve aus allen $n$ Messungen wird auf der Festplatte unter dem aktuellen Datum und dem Probennamen abgelegt.

d) Das Programm übergibt erneut an die Temperatursteuerung für den nächsten Temperaturpunkt und der Ablauf beginnt erneut von Punkt a).

7. Nachdem die Temperaturliste komplett abgearbeitet wurde, gibt das Programm eine Nachricht aus und die Messreihe ist beendet.

8. Werden viele Messungen unter Raumtemperatur durchgeführt, muss etwa stündlich der Dewar geprüft und ggf. nachgefüllt werden. Über Raumtemperatur ist kein Stickstofffluss nötig, die Heizer können jedoch auch ohne Probleme gegen den Stickstoff gegenheizen.

\section{A.4 Anleitung: Elektronenstrahlverdampfen}

Der Beschichtungsprozess mit der EBPVD-Anlage (siehe Kapitel 3.1.4) ist im Folgenden grob skizziert und kann in dieser Art als Anleitung der UHV-Anlage genutzt werden:

1. Die gereinigte Probe ${ }^{3}$ wird mit einer Klammer auf dem Probenhalter fixiert. Auf jeder Seite des Halters befinden sich je zwei Klammern mit je einem eingravierten Buchstaben (A,B,C und D). Dabei sind A B und C D auf einer Seite. Der Buchstabe wird zu jeder Probe notiert um die Position auf dem Halter nachverfolgen zu können.

2. Der Probenhalter wird an einer Haltestange getragen in das Schleusenkreuz der UHV-Kammer eingebracht und das Gewinde der Transferstage wird in den Probenhalter geschraubt. Anschließend kann die Haltestange abgeschraubt werden.

\footnotetext{
${ }^{3}$ Ultraschall: 1 min Aceton +1 min Isopropanol. Isopropanol mit Stickstoff herunterblasen.
} 
3. Der CF-Deckel der Schleuse wird locker auf die Vitondichtung aufgelegt (und wird nicht festgeschraubt). Das Sperrventil zwischen Schleusenturbopumpe und Schleusenvorpumpe wird geöffnet und beide Pumpen werden angeschaltet.

4. Nach 15-20 Minuten ist ein Druck unter $10^{-6}$ mbar erreicht und das Ventil zwischen Schleuse und Hauptkammer kann geöffnet werden.

5. Mit Hilfe der Transferstange wird der Probenträger in die Hauptkammer und auf die Halteraufnahme bewegt (zwei Dornen, gut durch eines der großen Fenster zu sehen). Für mehr Licht kann während des Schleusens die AML Heißkathode am Rack auf $1 \mathrm{~mA}$ gestellt werden (nach dem Schleusen sollte die Messröhre aber zurück auf $0.1 \mathrm{~mA}$ gestellt werden). Ist der Probenhalter auf die Aufnahme geschoben (Transferstange ist dabei am grünen Anschlagsring), kann die Transferstange vorsichtig aus dem Halter geschraubt werden. Es ist darauf zu achten, dass man mit dem letzten Gewindegang nicht noch am Halter hängt und ihn von der Aufnahme zieht.

6. Ist die Transferstange aus der Kammer gezogen (im Schleusen Fenster vergewissern!) kann das Ventil zwischen Schleuse und Kammer geschlossen werden. Der Kammerdruck sollte innerhalb weniger Minuten unter $5 \cdot 10^{-9}$ mbar fallen.

7. Alle genutzten Geräte müssen nun angeschaltet werden. Kühlwasserkreisläufe für Schwingquarz, Topf und Verdampfer müssen geöffnet sein. Des Weiteren muss der Inficon XTC/2 Schwingquarzmonitor muss für das zu verdampfende Material konfiguriert werden. Die materialspezifischen Parameter sind:

- Dichte $\rho$

- Z-Faktor: Ein Korrekturwert, welcher den Unterschied der akustischen Eigenschaften von Quarzkristall und aufgedampfer Schicht berücksichtigt.[149]

- Toolingfaktor: Der Unterschied zwischen der Aufdampfrate am Ort des Schwingquarzes und der Probe. Der Toolingfaktor muss in der Regel bei Änderungen an der UHV-Anlage oder bei Tausch des Verdampfermaterials neu bestimmt werden. Eine Anleitung zum Bestimmten des Toolingfaktors ist im XTC2-Handbuch finden. 
- PID Werte und maximale Verdampferleistung (optional).

8. Die gewünschte Schichtdicke wird im Computerprogramm oder am Inficon konfiguriert. Die Einheit ist $\mathrm{k} \AA$. Anschließend kann das Programm mit dem „Start Process“ Knopf im Steuerprogramm gestartet werden. Der Aufdampfprozess wird über in der Kammer angebrachte Spiegel durch ein Fenster beobachtet. Die einzelnen Prozessschritte sind die folgenden:

a) Rise 1: Die Leistung des Verdampfers wird in etwa einer Minute auf eine Leistung von etwa 60-70\% gefahren. Die Zeit und Leistung ist so eingestellt, dass das zu verdampfende Material kurz vor dem Schmelzpunkt ist.

b) Soak 1: Der Verdampfer hält die Leistung von Rise 1 für etwa eine Minute. In dieser Zeit soll sich das System gleichmäßig erwärmen.

c) Rise 2: Die Leistung wird auf einen Wert gefahren, an dem das Material aufschmilzt und eine Rate auf dem Schwingquarz abzulesen ist.

d) Soak 2: In dieser Phase wird die Leistung wieder konstant gehalten damit sich die Rate stabilisieren kann.

e) ShutterDelay: Der PID-Regelkreis des Inficon Schwingquarzmonitors übernimmt die Leistungssteuerung. Die Dauer dieser Phase richtet sich danach, wie gut Zeit und Leistung von Soak 2 gewählt wurden und wie schnell die gewünschte Aufdampfrate erreicht ist. Im Programm kann diese Phase mit dem „Hold“ Knopf beliebig gehalten werden um ggf. Probleme mit den Verdampfern oder dem Shutter zu lösen.

f) Deposition: Der XTC2 öffnet den Shutter und setzt die aufgezeichnete Schichtdicke auf 0. Der Aufdampfprozess hat begonnen. Ist die vorher eingestellte Schichtdicke erreicht, wird der Shutter automatisch geschlossen.

g) Idle Ramp: Die Leistung des Verdampfers wird langsam herunter gefahren. Ein zu schnelles Herunterfahren der Leistung könnte zu Rissen in Material und Tigel führen.

h) Idle: Der Verdampfer ist auf $0 \%$ Leistung und der Aufdampfprozess ist beendet. 


\section{A.5 Simulation mit dem Born-Von-Kármán-Modell}

In Abbildung A.3 ist die Benutzeroberfläche des Programms zum Zeitpunkt des Schreibens dieser Dissertation dargestellt. Anhand der Oberfläche möchte ich kurz die Funktionen der Simulationen beschreiben und anschließend die Details der Umsetzung beschreiben.
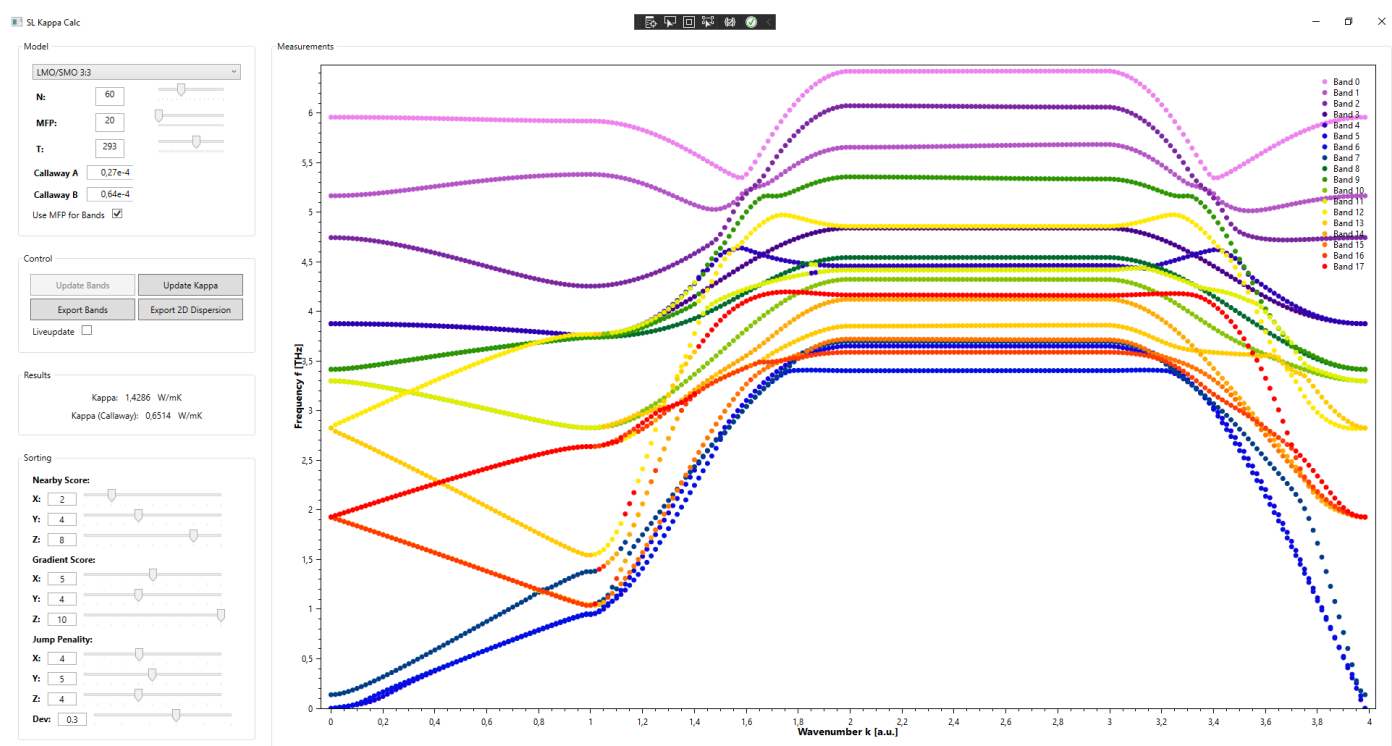

Abbildung A.3: Die Benutzeroberfläche der BvK-Simulation.

Der Simulationscode ist in $\mathrm{C} \#$ beschrieben. Die Gründe für diese Wahl waren unter anderem:

- Umfangreiche .NET Standardbibliothek und hilfreiche Zusatzbibliotheken wie z.B. MathNet zur Matrizenrechnung.

- In der Sprache integrierte Speicherverwaltung.

- Moderne und einfache Nutzeroberfläche durch Microsoft Windows Presentation Foundation.

- Einfache Parallelisierung durch async/await.

- Unterstützung durch Microsoft Visual Studio beim Schreiben des Programmcodes und Finden von Fehlern. 
Auf der linken Seite des Fensters (Windows Presentation Foundation WPF) sind die Eingabeelemente für die Simulationsparameter zu finden. Die oberste DropDown Liste listet alle registrierten Modell-Klassen (Klassen vererbt von der abstrakten Klasse Modell) auf. Darunter befinden sich Einstellungen für die Anzahl an diskreten Berechnungsschritten in jeder der drei Dimensionen. Also in wie viele Schritte die Achse im k-Raum $q_{x / y / z}=0$ bis $\pi / a_{x / y / z}$ eingeteilt wird. MFP ist die mittlere freie Weglänge aller Phononen entlang der $a_{z}$ Achse in Einheiten $1 / a_{z} . \mathrm{T}$ ist die simulierte Temperatur und Callaway A und Callaway B Parameter für ein Modell zur Berechnung der Wärmeleitfähigkeit/MFP nach Callaway [110].

Mit den Schaltfläche Update Bands werden die Bänder mit geänderten Parametern neu berechnet (was je nach Modell wenige Sekunden oder mehrere Minuten dauern kann) und mit Update Kappa wird die Wärmeleitfähigkeit der aktuellen Bandstruktur neu ausgerechnet um Änderungen an den Parametern T und Callaway A/B ohne Neuberechnung der kompletten Bandstruktur zu berücksichtigen.

Im rechten Fenster ist dann eine zweidimensionale Vorschau der Bandstruktur entlang Achsen hoher Symmetrie der 1. Brillouinzone von $[0,0,0] \rightarrow[0,0,1] \rightarrow$ $[1,0,1] \rightarrow[1,0,0] \rightarrow[0,0,0]$. Mit den Export-Schaltflächen lässt sich sowohl dieser zweidimensionale Schnitt als auch die echte dreidimensionale Bandstruktur aus dem Programm exportieren.

Die Umsetzung von Gleichung 2.31 in Programmcode ist in den Modellklassen gekapselt. Das Simulationsprogramm selbst erwartet nur eine Klasse mit der Implementation der abstrakten Klasse Model welche die Methoden

void ModelSetup (int $N$, double MFP)

und

Matrix<Complex> Solve(double qx, double qy, double qz)

implementieren muss. ModelSetup initialisiert die für die Berechnung verwendeten Listen zum Speichern von Zwischenergebnissen, welche abhängig von den Diskretisierungsschritten $N$ sind. Solve liefert die komplexe $3 k \times 3 k$ Matrix $\mathbf{M}(\vec{q})$ für die Position $\left(q_{x}, q_{y}, q_{z}\right)$ in der ersten Brillouinzone des simulierten Atomgitters.

Ein grober Programmablaufplan ist im Folgenden dargestellt:

- Erstellen aller Modellklassen und Auswahl einer Klasse durch den Benutzer. 
- Aufruf von ModelSetup: Festlegen der Unterteilung der Brillouinzone in $N$ diskrete Schritte je Dimension.

- Aufruf von Solve für jeden Punkt in der 1. Brillouinzone.

- Die Eigenwerte des Rückgabewerts von Solve (also die komplexe Matrix $\mathbf{M}\left(q_{x}, q_{y}, q_{z}\right)$ werden mittels MathNet gelöst und in einer Liste gespeichert.

- Die Eigenwerte sind nach Energie und nicht nach Bandindex sortiert, was an Schnittstellen von Bändern zu (unphysikalischen) Unstetigkeiten führt. Die Werte werden daher zunächst auf ihre entsprechenden Bänder sortiert.

- Für die eindimensionale Wärmeleitfähigkeit $\kappa$ wird nun der Gradient in zRichtung eines jeden Bandes aufsummiert.

Eine allgemeine Implementierung für beliebige Übergitter hat sich als schwierig heraus gestellt, weshalb zunächst separate Klassen für die Modelle der Übergitter $m / n=1$ und $m / n=2$ geschrieben wurden. Das Gleichungssystem $\mathbf{M}\left(q_{x}, q_{y}, q_{z}\right)$ lässt sich in drei Untermatrizen der Größe $k \times k$ aufteilen. Die Elemente dieser Matrizen lassen sich durch einzelne Schleifen über die 6 nächsten, 12 übernächsten und 8 über-übernächsten Nachbarn berechnen. 


\section{A.6 Phononendispersion aus dem BK-Modell}

Im Folgenden liste ich die mit dem BK-Modell berechneten Phononendispersionen für die in dieser Arbeit untersuchten LMO/SMO-Übergitter auf.

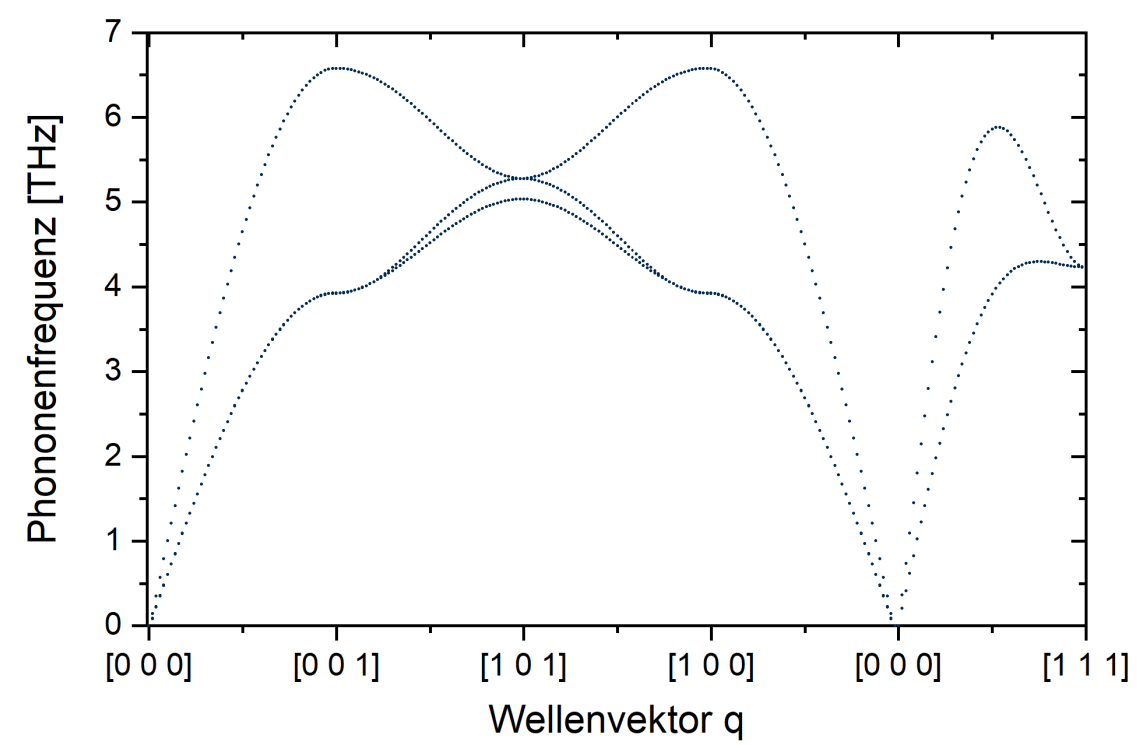

Abbildung A.4: Phononenbandstruktur von kubischem ( $\mathrm{Pm} \overline{3} \mathrm{~m})$ LMO berechnet mit dem BK-Modell. 


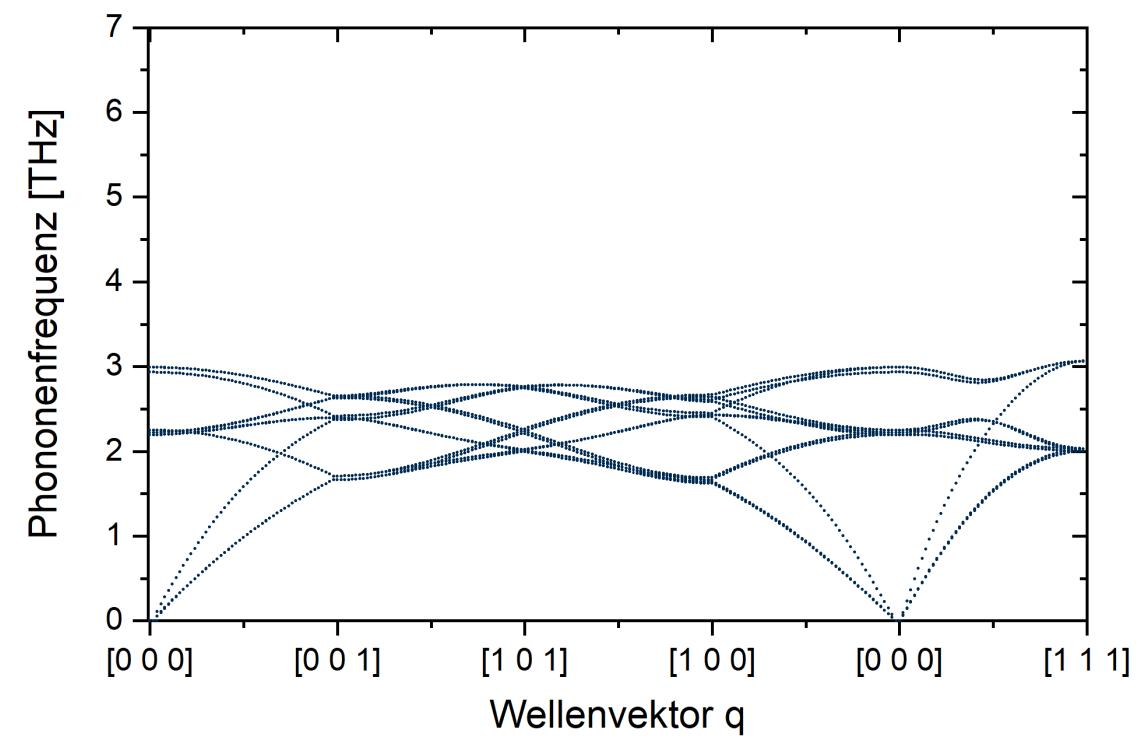

Abbildung A.5: Phononenbandstruktur von rhomboedrischem (R $\overline{3} \mathrm{c})$ LMO berechnet mit dem BK-Modell. Die (R $\overline{3} \mathrm{c}$ ) LMO Struktur wurde im BK-Modell durch eine LMO-Superzelle berücksichtigt. Um der unterschiedliche Orientierung und Kopplung benachbarter LMO-Zellen Rechnung zu tragen, haben wir die Masse der LMO-Einheitszellen in der Superzelle periodisch variiert. Die Variation der Masse haben wir mit Hilfe der $m / n=2$-Übergitter an die Messdaten angepasst. Die Wärmeleitfähigkeit aus dem BKM für rhomboedrisches LMO decken sich mit Angaben von [109]. 


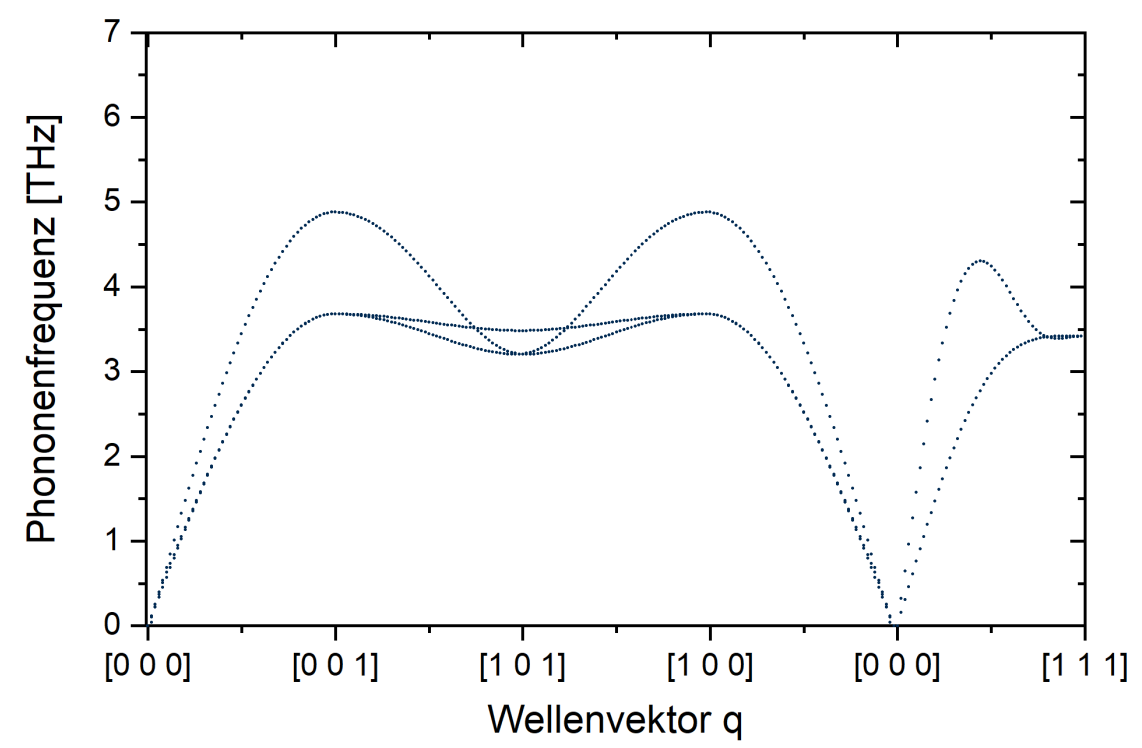

Abbildung A.6: Phononenbandstruktur von kubischem ( $\mathrm{Pm} \overline{3} \mathrm{~m})$ SMO berechnet mit dem BK-Modell.

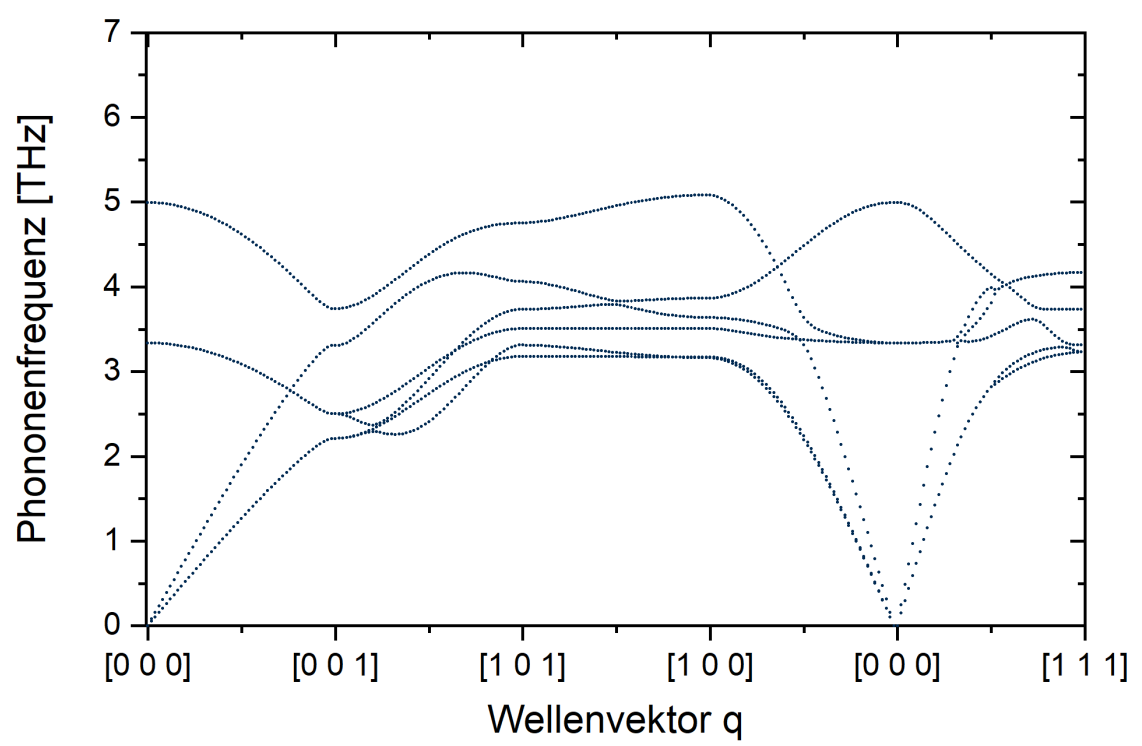

Abbildung A.7: Phononenbandstruktur von $(\mathrm{LMO})_{1} /(\mathrm{SMO})_{1}$ berechnet mit dem BK-Modell. 


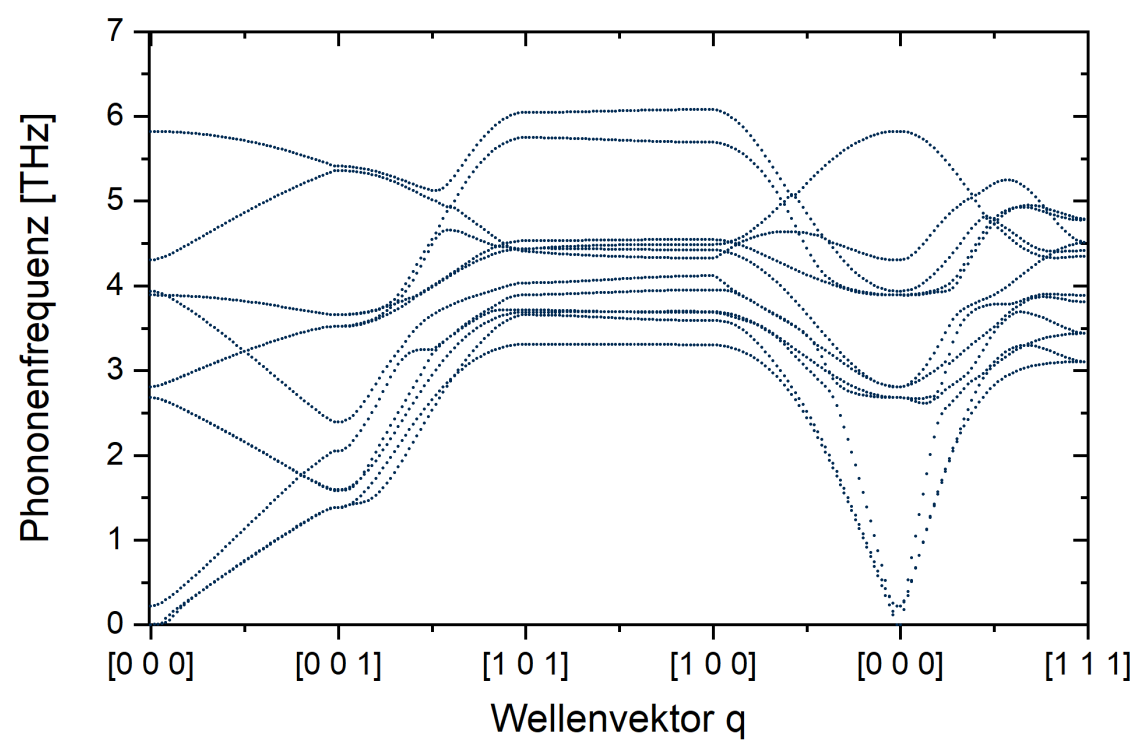

Abbildung A.8: Phononenbandstruktur von $(\mathrm{LMO})_{2} /(\mathrm{SMO})_{2}$ berechnet mit dem BK-Modell.

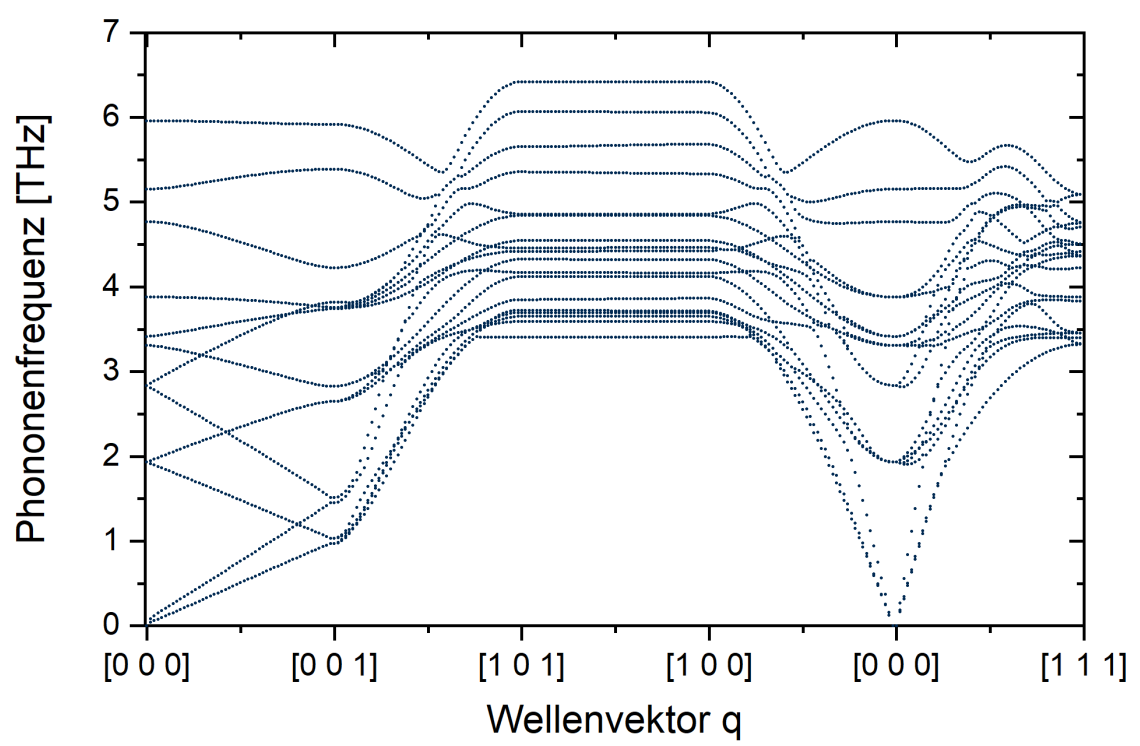

Abbildung A.9: Phononenbandstruktur von $(\mathrm{LMO})_{3} /(\mathrm{SMO})_{3}$ berechnet mit dem BK-Modell. 


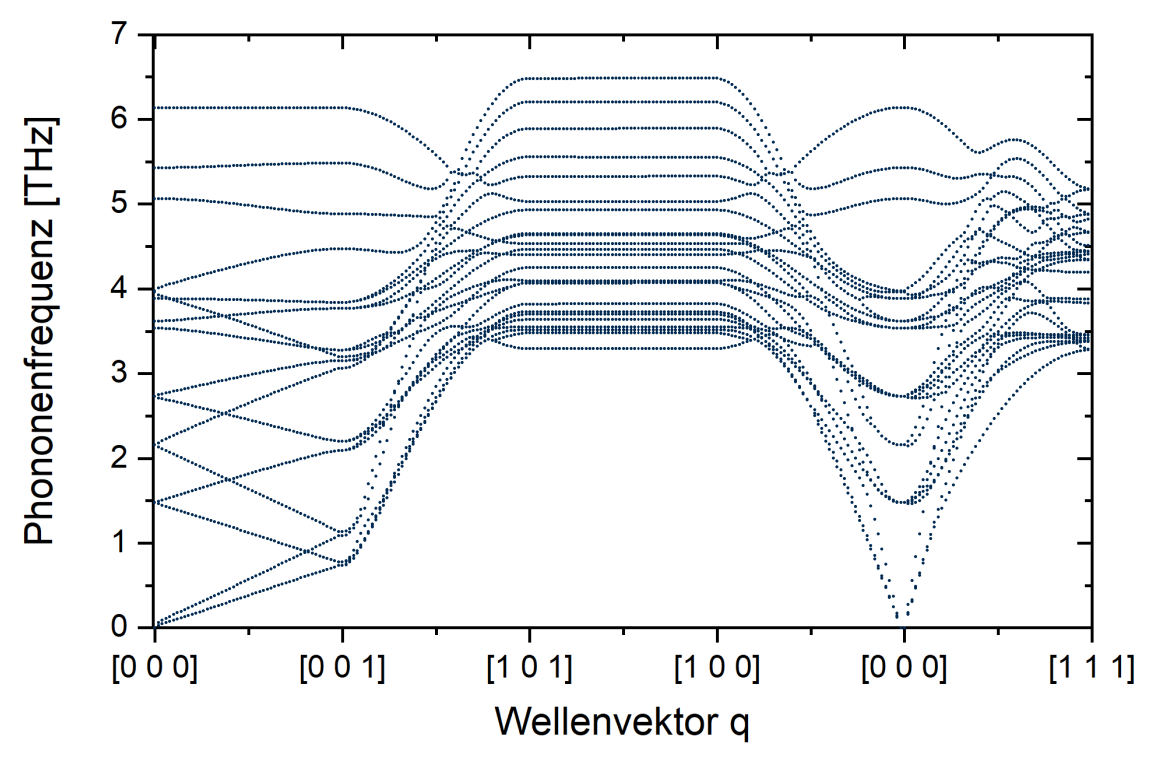

Abbildung A.10: Phononenbandstruktur von $(\mathrm{LMO})_{4} /(\mathrm{SMO})_{4}$ berechnet mit dem BK-Modell.

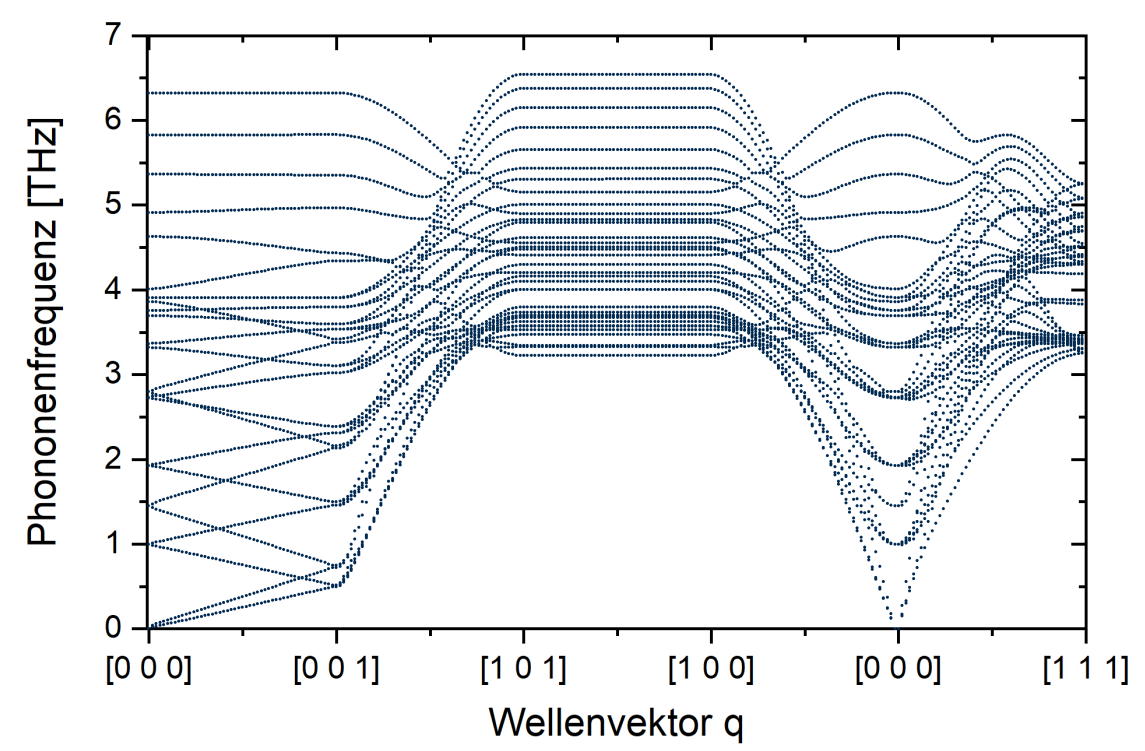

Abbildung A.11: Phononenbandstruktur von $(\mathrm{LMO})_{5} /(\mathrm{SMO})_{5}$ berechnet mit dem BK-Modell. 


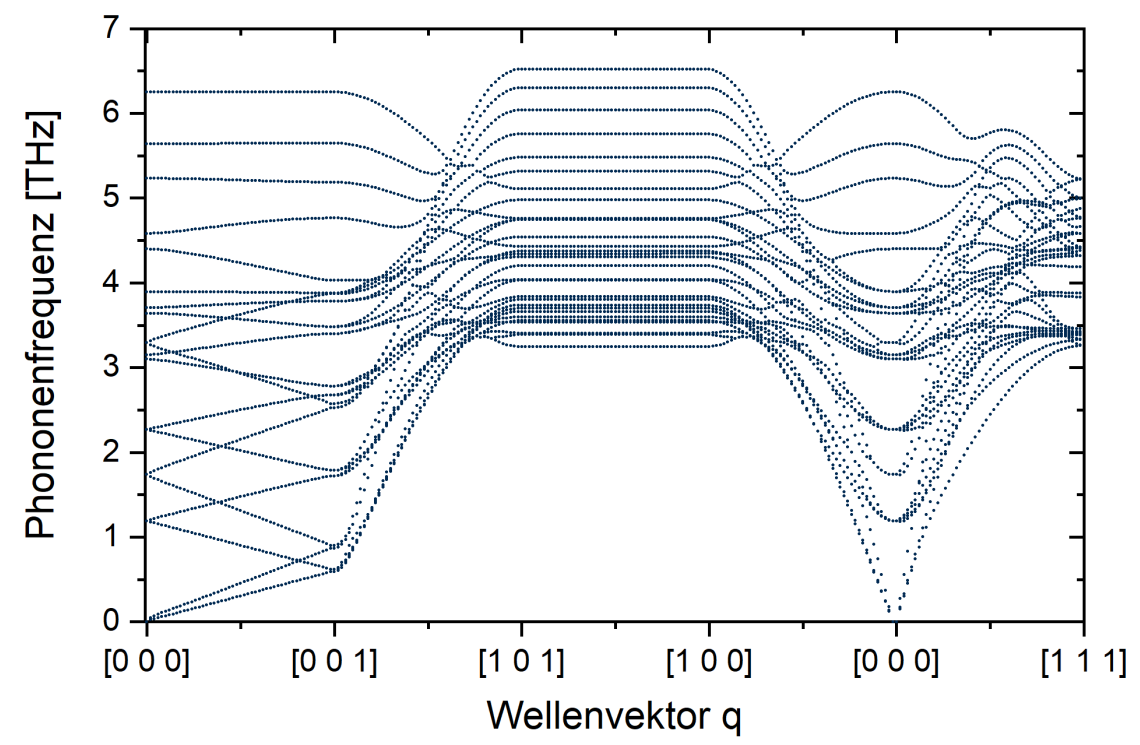

Abbildung A.12: Phononenbandstruktur von $(\mathrm{LMO})_{6} /(\mathrm{SMO})_{6}$ berechnet mit dem BK-Modell.

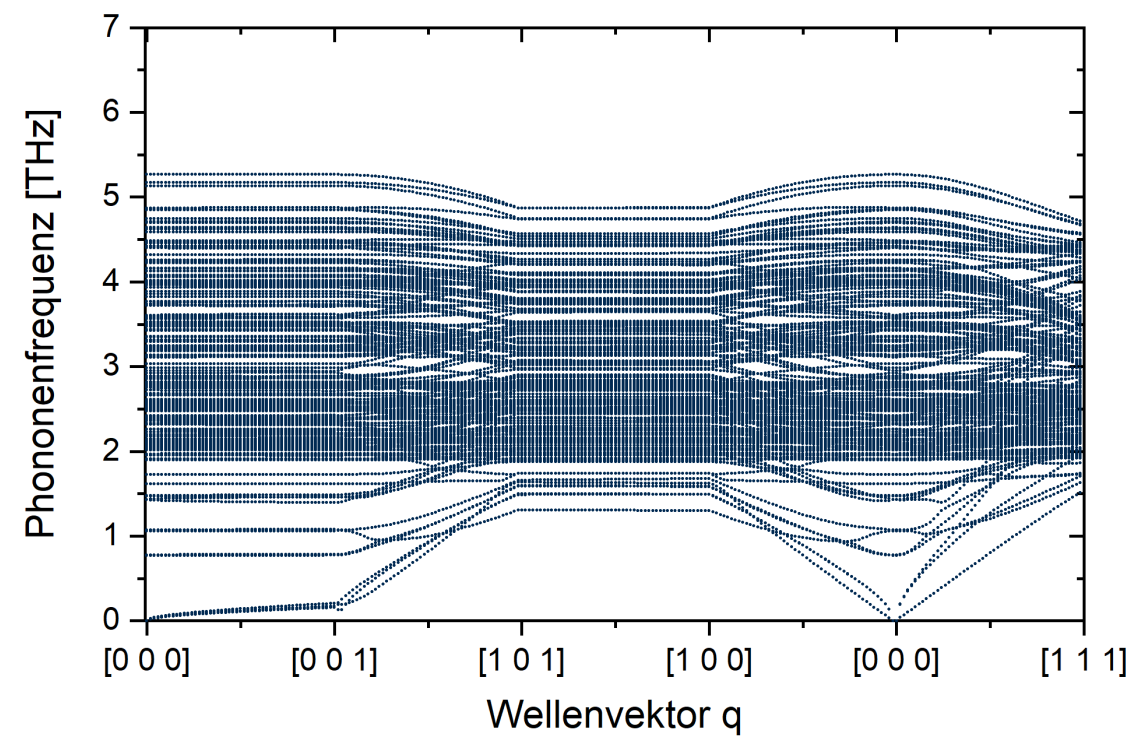

Abbildung A.13: Phononenbandstruktur von $(\mathrm{LMO})_{10} /(\mathrm{SMO})_{10}$ berechnet mit dem BK-Modell. 


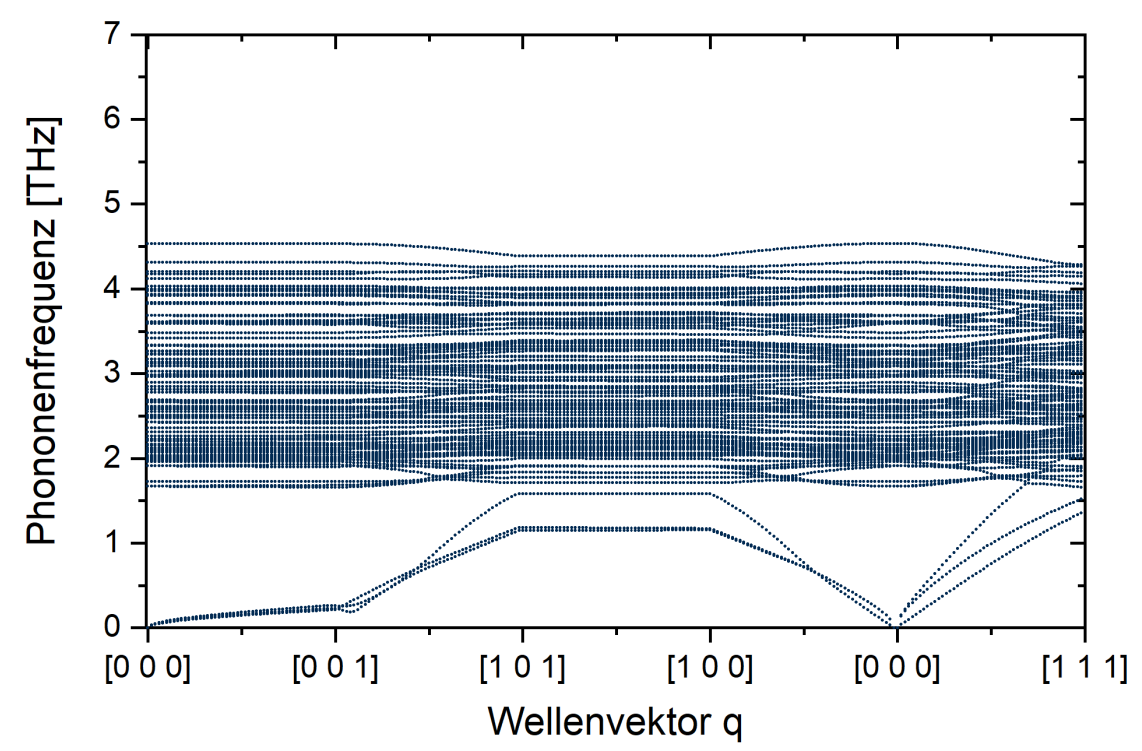

Abbildung A.14: Phononenbandstruktur von $(\mathrm{LMO})_{8} /(\mathrm{SMO})_{4}$ berechnet mit dem BK-Modell.

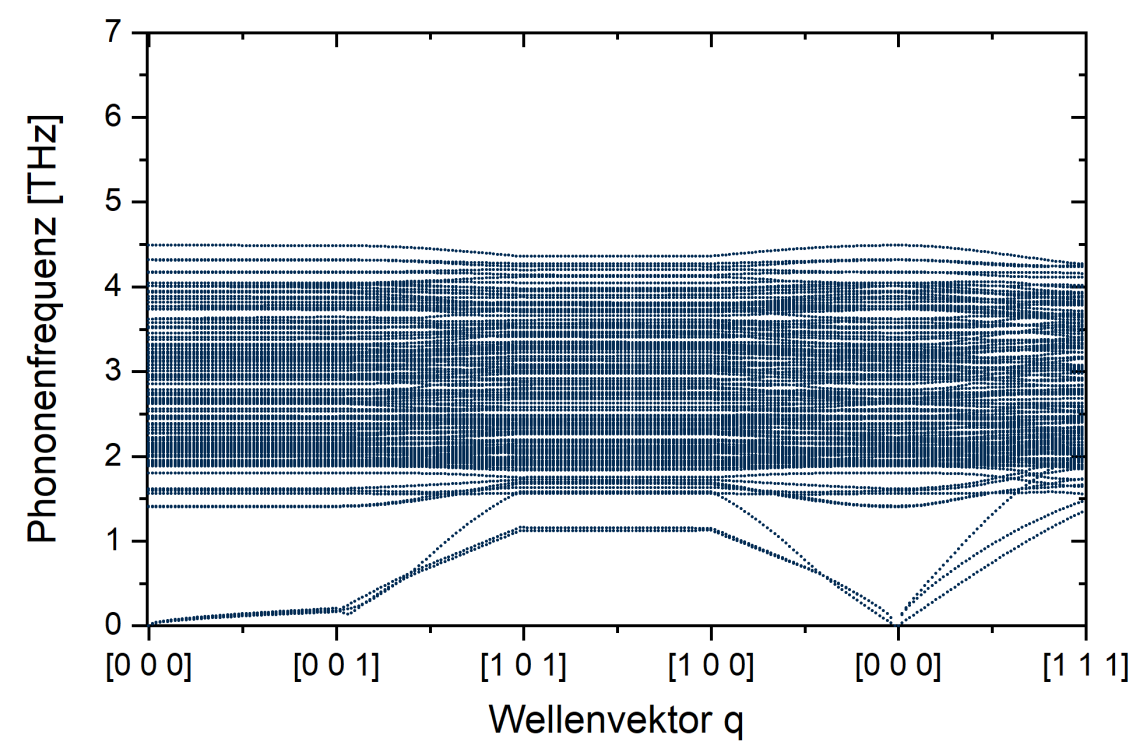

Abbildung A.15: Phononenbandstruktur von $(\mathrm{LMO})_{10} /(\mathrm{SMO})_{5}$ berechnet mit dem BK-Modell. 